\title{
Living Well in a World With Wolves: Educators' Perspectives
}

\author{
Gail J. Kuhl \\ Faculty of Education \\ Lakehead University \\ Thunder Bay, Ontario
}

February 2016

A dissertation submitted in partial fulfillment of the requirements of the degree of Doctor of Philosophy in Educational Studies 


\begin{abstract}
The purpose of this qualitative, phenomenological investigation was to explore wolf-focused education from the perspective of educators who work at various wolf education centres and programs around North America. The research question was: What are the experiences of educators who work at programs that feature captive or wild wolves; in particular, what do these educators learn through both working with and teaching about wolves? The research sought: to better understand the learning that emerges from direct experiences humans have with other animals, in this case wolves; to fill a gap in the research on wolf education; and to explore and understand some of the ethical aspects of wolf education and wolf conservation from the perspective of educators. Using a phenomenological methodology that drew from critical theory, methods included 16 in-depth semi-structured interviews with 17 educators from 15 different wolf programs around North America, a brief content analysis of the programs' websites, and observations of two wolf programs. Topics discussed with the educators included the purpose of their work, their ideas about how best to educate the public about wolves, their own ideas about wolves including what they had learned through observing and working with wolves, and their thoughts on wild wolf management and conservation. When it came to the experience of being a wolf educator certain motifs emerged that help elucidate the experience: the significance of political, regional, and cultural contexts; the key role that scientific knowledge and learning has for many wolf educators; the importance of ethics to the educators when it comes to teaching about and working with captive or wild wolves; the impact of direct experiences with wolves on how educators understand and feel about wolves; and finally, their engagement with conservation issues.
\end{abstract}


This research highlights the importance of education as one strategy for wolf conservation, especially if it does not shy away from the messiness of controversial conservation issues and helps people grapple with the social, ethical, regional, and cultural aspects of knowing wolves and our relationships as humans with them. 


\section{ACKNOWLEDGMENTS}

I would like to thank my supervisor Dr. Connie Russell for ten years of mentorship. She is a gifted advisor and I would not have completed this program without her support, encouragement, and inspiration throughout. She was integral to all stages of this dissertation from conception to final edits.

I would also like to thank my committee members Dr. David Greenwood and Dr. Mary Breunig for their thoughtful reading and responses to my work both in the early and later phases of my dissertation. Their feedback definitely shaped the eventual outcome especially with regards to how I envisioned and presented my methodology and methods, and some of the more philosophical avenues I chose to explore. Thank you also to Dr. Hilary Brown for coming on to my committee at the end and providing some useful feedback and support.

Importantly, I would like to thank all the dog friends and companions I've shared my life with over the years. They have inspired me to want to explore human-animal relations on a broader scale in the first place, and have transformed my personal beliefs and ethics around how humans should/could live better with our animal neighbours.

I would like to thank the Social Sciences and Humanities Research Council of Canada (SSHRC) for a PhD fellowship that helped fund and make this research possible.

Finally, I am grateful to my parents Josephine Kuhl and Roger Kuhl for always being supportive and encouraging of my seemingly endless academic pursuits. My mom would have loved to see the end of this $\mathrm{PhD}$ process, but I'll do my best to bring her to the finish with me. Her curious nature, continual desire to learn new things, and her passion for education have clearly left their mark on me. 


\section{TABLE OF CONTENTS}

ABSTRACT

\section{CHAPTER ONE:}

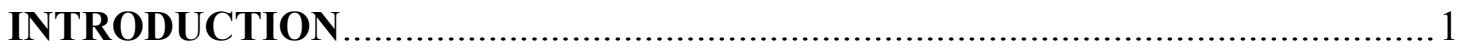

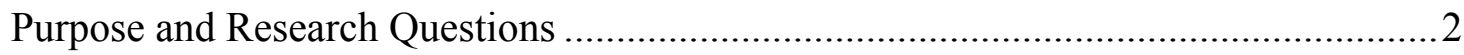

Definition of Key Terms …………………………….......................................

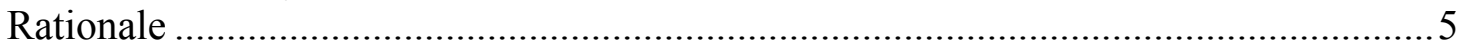

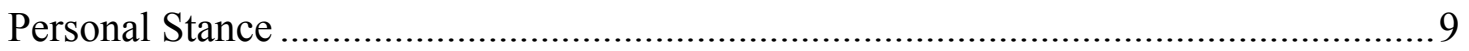

\section{CHAPTER TWO:}

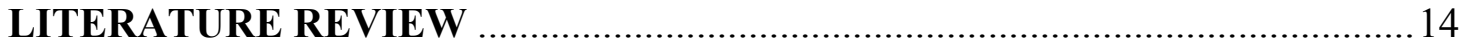

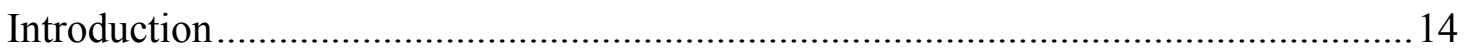

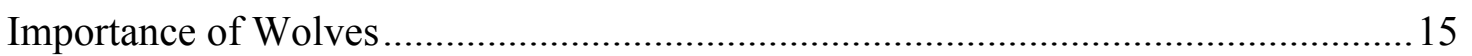

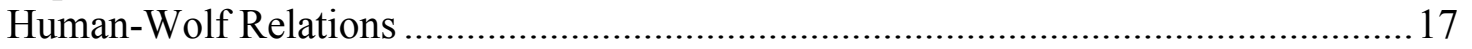

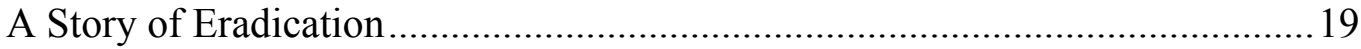

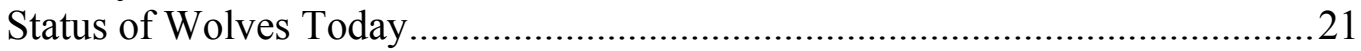

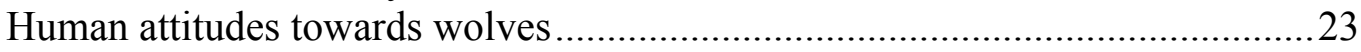

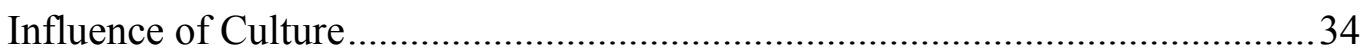

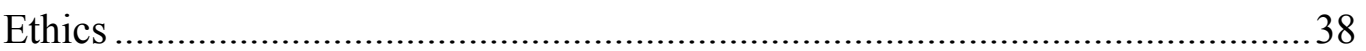

Environmental and Humane Education ................................................................... 43

Environmental Education Overview …………………....................................4

Humane Education Overview..........................................................................4

Environmental Education Research Focussing on Wildlife.................................49

Humane Education Research.......................................................................59

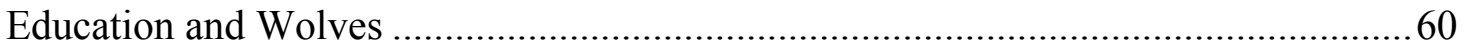

Literature Specific to Wolf Education................................................................... 61

Research and Literature in Which Wolf Education is a Component.....................66

\section{CHAPTER THREE:}

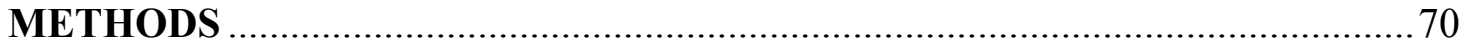

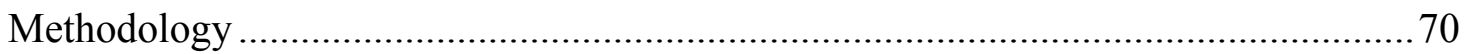

Qualitative Study Design............................................................................... 70

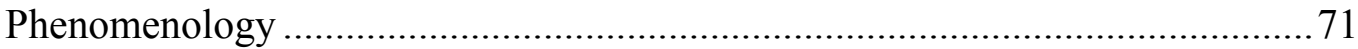

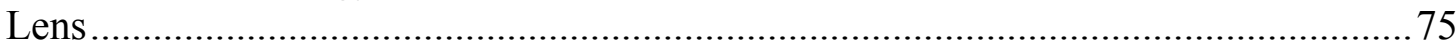

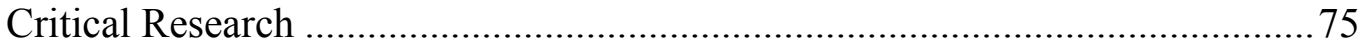

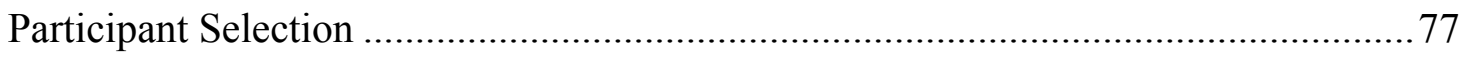

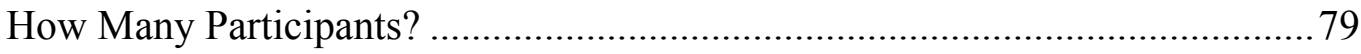

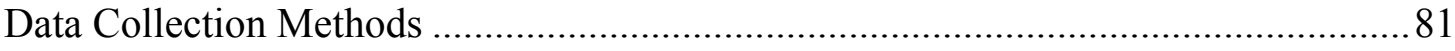

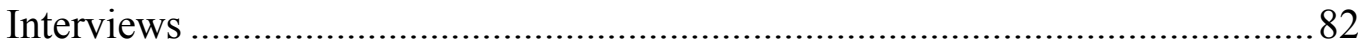

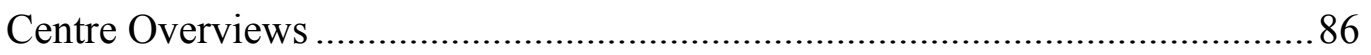




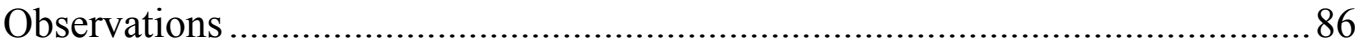

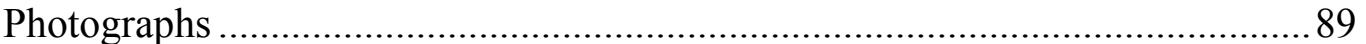

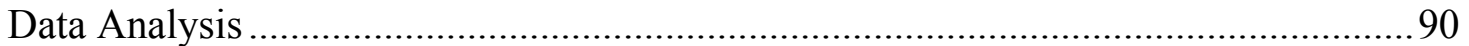

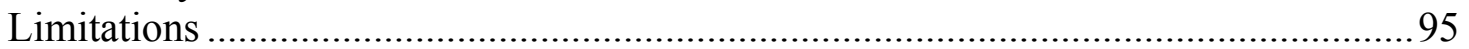

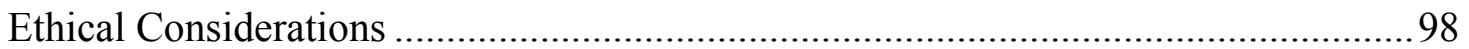

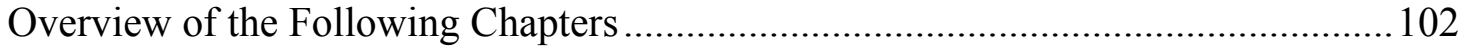

\section{CHAPTER FOUR:}

PARTICIPANT AND CENTRE OVERVIEWS.................................................104

Participant Overview ............................................................................................ 104

The Path to Becoming a Wolf Educator........................................................ 105

What Wolf Educators Do: Tasks .................................................................. 108

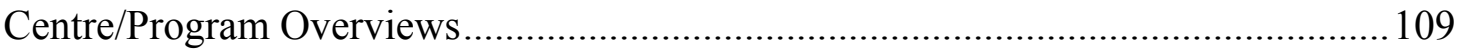

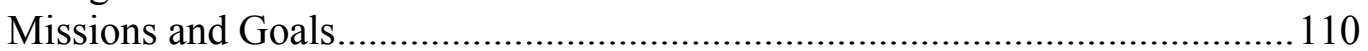

Organizations' Details .................................................................................... 111

Programming ................................................................................................ 112

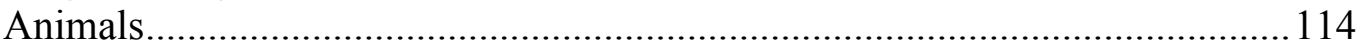

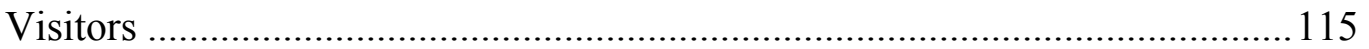

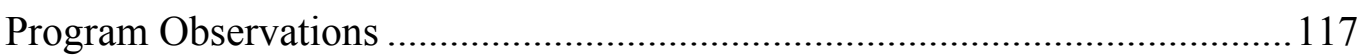

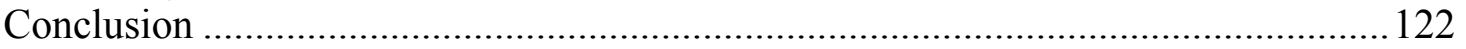

\section{CHAPTER FIVE:}

THE COMPLEXITIES OF EDUCATION FOR CHANGE …………………..... 123

What Kind of Change? The Purpose of Wolf Education.............................................123

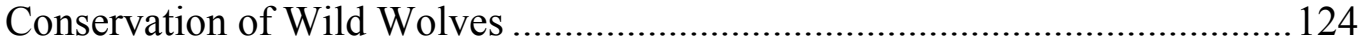

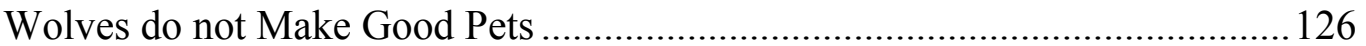

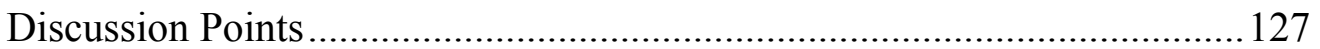

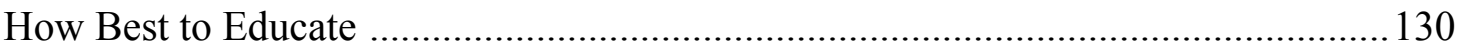

Advocacy Versus Sharing Science .................................................................. 131

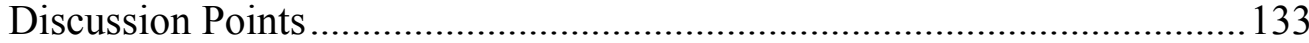

Meet People Where They Are At: Dialogue and Listening ..............................135

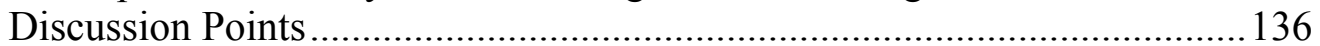

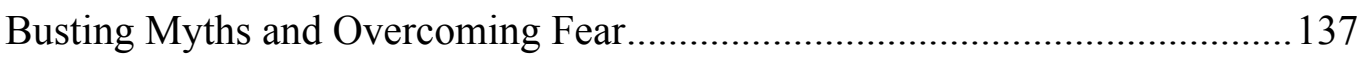

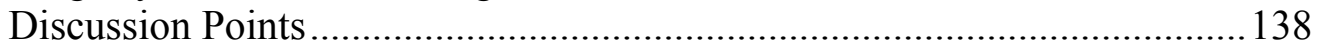

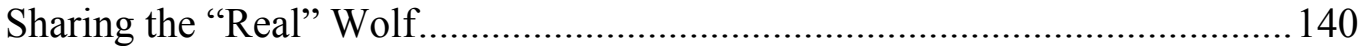

Discussion Points ................................................................................ 141

Making Connections: Wolves are Like People, Wolves are Like Dogs ............141

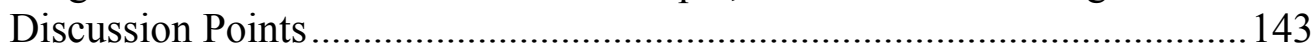

Meaningful Experiences and Emotional Hooks .................................................. 145

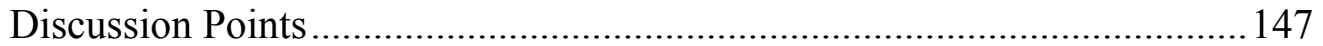

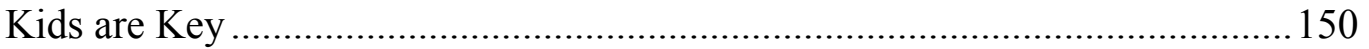

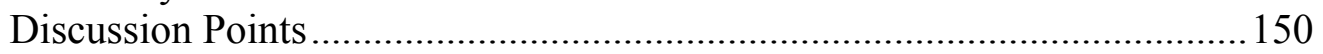

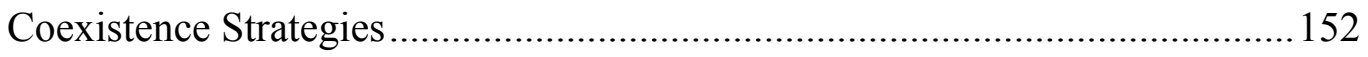

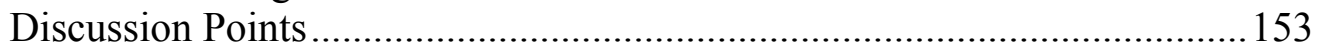

The Challenges and Rewards of Being a Wolf Educator ............................................ 154

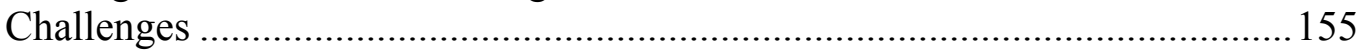




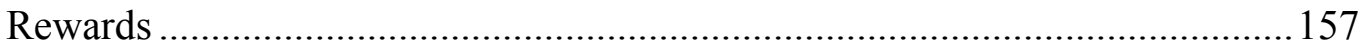

Challenges and Rewards: Discussion Points ................................................... 158

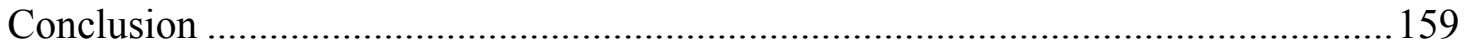

\section{CHAPTER SIX:}

UNDERSTANDING AND WORKING WITH WOLVES ...................................161

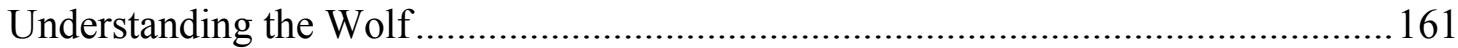

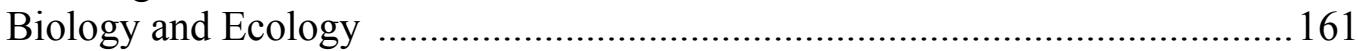

Understanding the Polarity of Human-Wolf Relations .......................................162

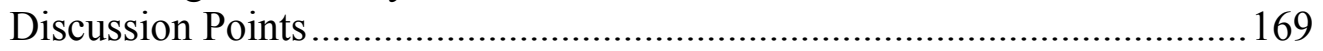

Understanding the Wolf Through Experience.................................................... 171

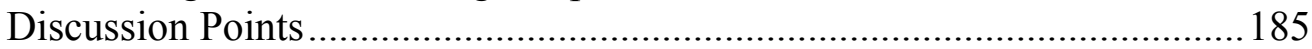

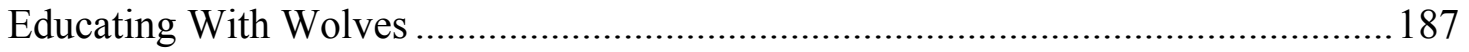

Experiencing Wolves: Perceived Benefits for Participants............................... 188

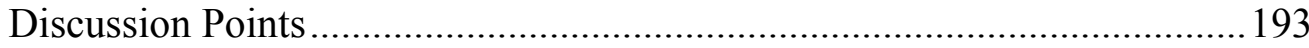

Wolves in Education: Ethical Aspects ...........................................................195

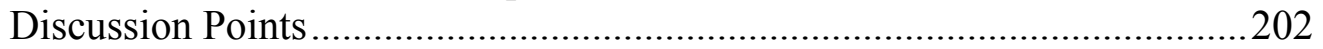

How to Care for Captive Wolves ................................................................220

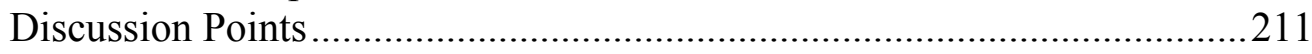

Elements of Educator-Wolf Relationships ....................................................212

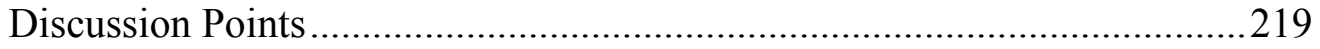

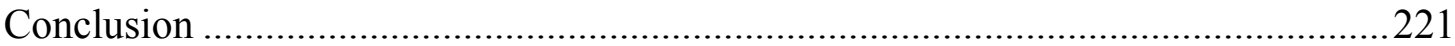

\section{CHAPTER SEVEN:}

ENGAGING IN THE CONTROVERSY OF WOLF CONSERVATION ..........224

Why Wolves Matter ............................................................................................22

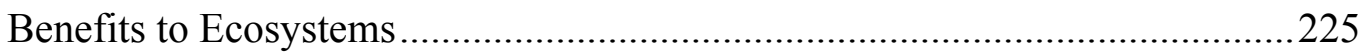

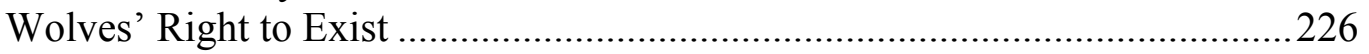

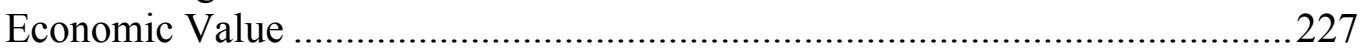

Why Wolves Matter: Discussion Points.........................................................222

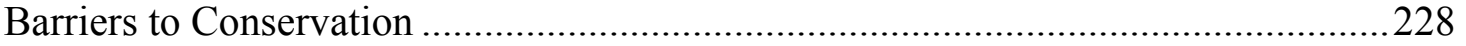

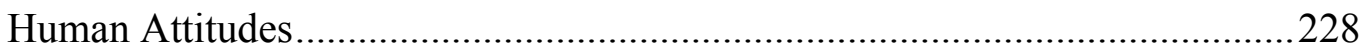

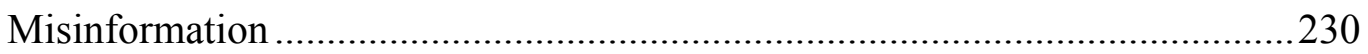

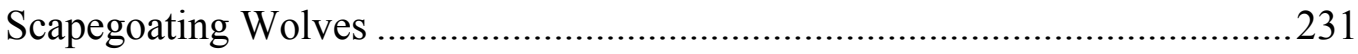

Politics and Legislation ..............................................................................233

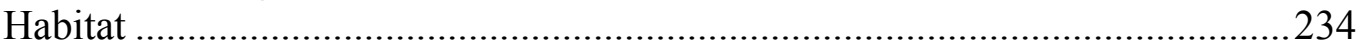

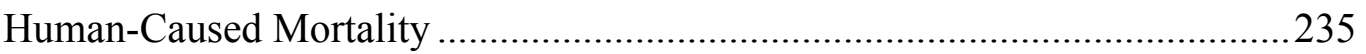

Barriers to Conservation: Discussion Points .....................................................2235

The Ethics of Conservation and Management ............................................................237

Humans Should Adjust to Living with Wolves................................................237

Management Should Account for Wolves' Social Nature .................................2239

Science-Based Decision-Making.....................................................................2240

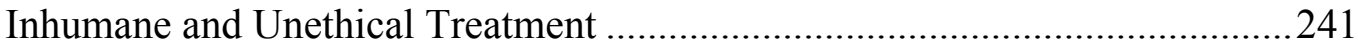

The Ethics of Wolf Management: Discussion Points.........................................2243

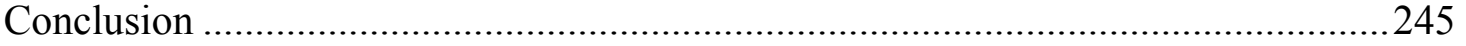




\section{CHAPTER EIGHT:}

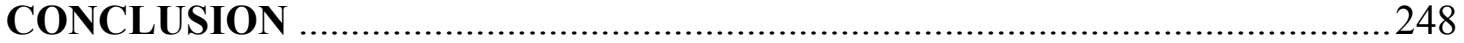

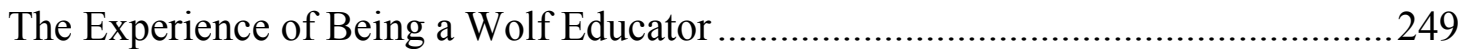

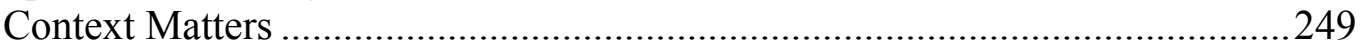

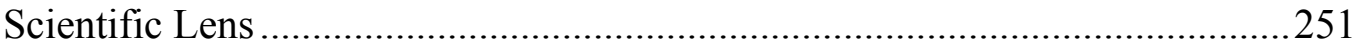

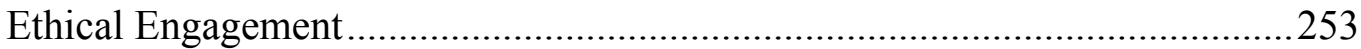

Learning About Wolves through Experience ..............................................255

Wolf Education for Wolf Conservation ........................................................2258

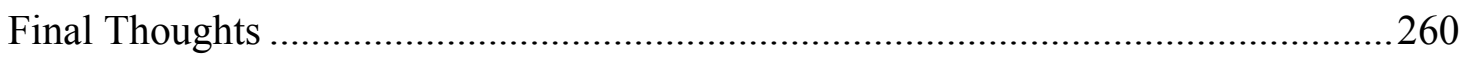

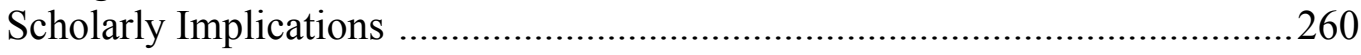

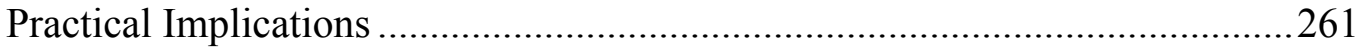

Wolf Education for Wolf Conservation .........................................................263

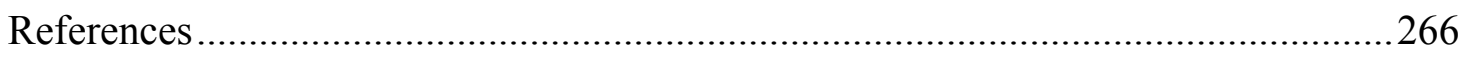

Appendix A: List of Possible Interview Questions for Wolf Educators ...................283

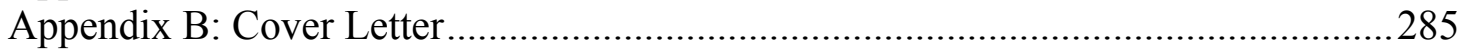

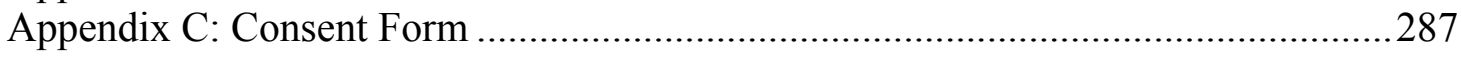

Appendix D: Consent Form for Use of Photographs ..........................................28 


\section{LIST OF TABLES AND FIGURES}

Tables

Table 1. Example of Theme Development ............................................................94

Table 2. Participant Overview 107

Figures

Figure 1. Algonquin Provincial Park Wolf Howl .................................................. 146

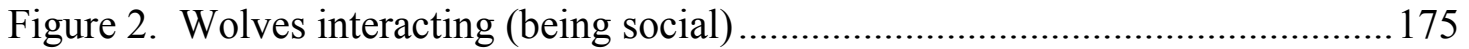

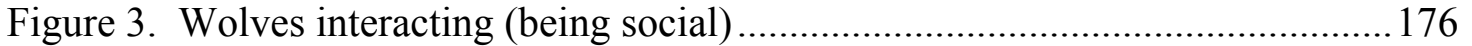

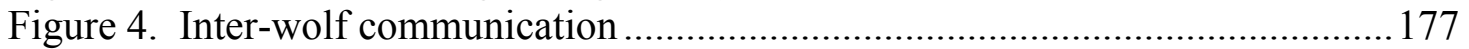

Figure 5. Inter-wolf communication ............................................................... 177

Figure 6. Inter-wolf communication ............................................................... 177

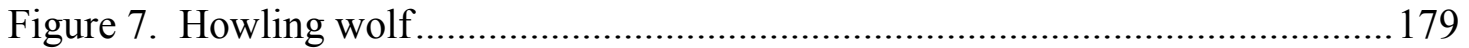

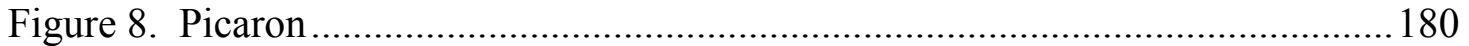

Figure 9. Photograph of wolf paw prints with a human handprint ......................... 188

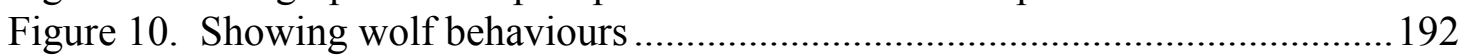

Figure 11. Socialized wolves with handler at Wolf Park....................................... 196

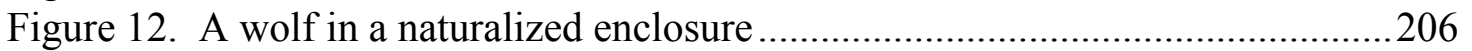

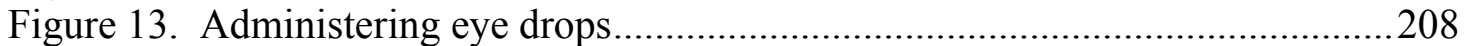

Figure 14. Healthcare with socialized wolves ......................................................208

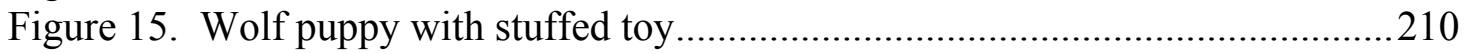

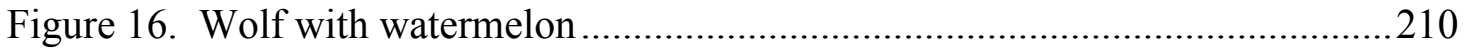

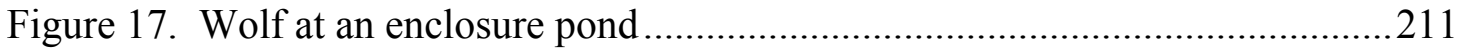

Figure 18. Wolf with fish from enclosure pond.....................................................211

Figure 19. Michelle and Kwahadi, one of her favourite wolves..............................214

Figure 20. Monty photographing the pack.............................................................. 215 


\section{CHAPTER ONE \\ INTRODUCTION}

Not a week goes by that I do not see a headline about wolves, such as "National Geographic puts spotlight on B.C.'s enigmatic sea wolves” (Talmazan, 2015), “Import Wolves to Michigan's Isle Royale ‘as soon as possible,' says biologist” (Ellison, 2015), and "Wolf-culling policies need updating, Alberta conservationist says" (Edmonton AM CBC, 2015). We share our planet with many animals; some we rarely think about. Wolves are not one of these, rather they are frequently in the spotlight, their existence often arousing passionate responses in people (both negative and positive) and causing controversy (Chadwick, 2010).

While in parts of North America wolves have co-existed alongside humans for centuries, in the last 40 years they also have been reintroduced to and/or reinhabited regions where they were once extirpated (Boitani, 2003). In those areas, educational efforts were often developed, in part, to help improve attitudes and ease the public's transition into once again sharing a world with wolves (Fritts, Stephenson, Hayes, \& Boitani, 2003; Troxell, Berg, Jaycox, Strauss, Shruhsacker \& Callahan, 2009). Despite the fact some of these educational efforts have been around for years, from a research perspective, little is known about either wolf-focused educational programs or the people who deliver them. And this brings me to my research topic. This dissertation explores, though interviews and observations, the experiences of 17 individuals in North America who educate people about wolves. In this first chapter, I describe the purpose and research questions, define key terms, and provide a rationale for my dissertation as well as outline my personal stance to this research. 


\section{Purpose and Research Questions}

The purpose of this qualitative investigation was to explore wolf-focused education from the perspective of educators who worked at different wolf education programs around North America. I embarked on the research, asking: What are the experiences of educators who work at programs that feature captive or wild wolves; in particular, what do these educators learn through both working with and teaching about wolves?

The following sub-questions guided me in answering the overarching research question above:

- What are the key messages that educators hope to offer participants in their programs?

- How has working with and teaching about wolves had an impact on educators' personal attitudes and beliefs about wolves?

- What do educators learn through working with wolves at wolf programs, both about wolves and from the wolves themselves?

- What ethical issues, if any, are raised for them in using wolves in education programs?

- Do educators think education about wolves makes a difference when it comes to conservation? Why or why not? If yes, how so?

Through conducting this study, I also hoped to grapple with part of a larger question:

What is it that we need to know as humans to coexist and live well in a world with wolves?

Why it is important to understand wolf education and human-wolf relations better in the first place? In the following in-depth discussion, I argue that more research is needed that acknowledges the sentience of other animals and explores the learning that comes from our relationships with them. Some people still question the value of 
conserving wolves, and in many regions where wolves reside, human-wolf conflicts abound (Bisi, Kurki, Svensberg, \& Liukkonen, 2007; Chadwick, 2010; Fritts, et al., 2003). Still, there are a number of reasons for ensuring their continued well-being including: their important role in healthy ecosystems (Eisenberg, 2010; Ripple et al., 2014), their cultural and heritage importance, their status as a symbol of wilderness (Ontario Ministry of Natural Resources, 2005), as well as their intrinsic value and right to exist when considered from an ethical and moral standpoint (Fox \& Bekoff, 2009; Lynn, 2007). Education's role in helping people live peacefully in a world with wolves has barely been researched; in fact, I uncovered only three studies that specifically examined wolf education (i.e. Black \& Rutberg, 2007; Samuelson, 2012; Willard, 2008). Honing in of the experiences of wolf educators, this in-depth investigation helps fill a gap in our understanding of wolf education and some aspects of human-wolf relations.

\section{Definition of Key Terms}

Before I continue with the details of the study, I will define some key terms. Many of these are discussed in more depth in the literature review and methodology sections that follow, but these operational definitions should provide a starting point.

Wolf Education Program: For the purposes of this research, a "wolf education program" is any program dedicated to educating the public about wolves. There are many such programs worldwide, both formal and informal, and these programs take many forms including: informational websites, educator-led workshops, sessions and presentations, travelling exhibits, curriculum resources, teacher workshops, films, 
educational displays, wolf viewing, and other direct experiences with wolves like wolf walks/howls (Troxell, et al., 2009; Willard, 2008).

Wolf Centre: Similar to a wolf program, a wolf centre is dedicated to wolf education, but in this instance, there is an actual building or facility that houses the program. Two examples are The International Wolf Center in Ely, Minnesota, and Wolf Park in Indiana (Troxell et al., 2009).

Wolf Educator: An individual who spends time (paid or volunteer) teaching the public about wolves.

Captive and Wild Wolves: For the purposes of this study, I chose to interview only those educators who worked at programs that involved live wolves. In this research, that included wild wolves such as those encountered at the wolf-howling program in Algonquin Park in Ontario (The Friends of Algonquin Park, 2014), but more often, the wolves were captive. Some programs, for example, have enclosures where the wolves reside and a viewing area for the public (Troxell, et al., 2009).

Direct Experience: As it pertains to this investigation, direct experience refers to "embodied, sensory" and "first hand" encounters with other animals (Fawcett, 2002, p. 126). In particular, I explored what educators learned from either howling with wild wolves or interacting with captive wolves through feeding and other care activities, socializations, and educational programs.

Humane Education: Contemporary humane education focuses on providing information about the situation of other animals and developing a broad range of skills and capacities such as curiosity, creativity, critical thinking, kindness, respect, reverence, 
and responsibility with respect to other animals, hopefully leading to empowering learners to make choices that benefit the earth and all its animal inhabitants (Weil, 2004).

Environmental Education: Environmental education can be loosely defined as an interdisciplinary field of education focused on "the nature of people-societyenvironment relationships" (Stevenson, Wals, Dillon, \& Brody, 2013, p. 2) in both informal and formal settings, and ranging in orientation from the local to the global. It may focus on our attitudes, values, knowledge, and understanding of environmental issues and concerns as well as "developing the agency of learners in participating and taking action" (Stevenson et al., 2013, p. 2).

Anthropocentrism: "Anthropocentrism refers to the consideration of humans as the center of all valuing" (Fawcett, 2013, p. 409), more specifically, that the environment and other species only have worth in so much as they "fulfill human needs, desires and goals" (Fawcett, 2013, p. 410).

\section{Rationale}

I embarked on this research with the premise that through working with wolves, educators may learn things not only about, but also from members of this other species. Considering the lack of research investigating wolf education in general and the experiences of wolf educators in particular, I believed that there were insights to be gained into the ethical aspects of what it means to teach and learn about wolves and their conservation.

Thinking about cross-species learning, I set out on this study suspecting that not only do participants in wolf education programs have potential to learn about wolves and 
wolf conversation, but that the wolf educators themselves learn much through working directly with wolves. Liddicoat and Krasny (2013) review a substantial number of studies that indicate that firsthand experiences in the natural world, both formal and informal, may lead to outcomes such as environmental awareness, pro-environmental attitudes and behaviour, and/or environmental knowledge; however, there is little research considering the educational ramifications of direct experiences specifically with other animals as one particular part of nature.

An example of research that does consider the learning that arises through direct interspecies encounters is my own master's thesis on human-sled dog relations, where I found that the mushers gained important understandings (e.g. about empathy, patience, abilities of another species) through interacting daily with sled dogs (Kuhl, 2011a). Other researchers have also concluded that direct experiences with other animals can have learning benefits. Fawcett (2002), for example, found that a brief direct experience with a wild animal led kindergarten children to both feel more positive about the species and be more likely to tell kinship and friendship stories involving the animal. Similarly, Ross, Medin, Coley, and Atran (2003) found that children who had more day-to-day interaction with nature developed less anthropocentric and more ecocentric reasoning about non-human animals. Prokop and Tunnicliffe (2010) surveyed children and discovered that having various animals at home (pets and farm animals) correlated with improved attitudes towards and knowledge about wild animals, both those considered popular and unpopular like wolves. Similarly, Fidler, Light, and Costall (1996) found that growing up with a cat or dog changed how participants described dog behaviours in video clips; for example, the behaviours were more often described in terms of the 
animal's desires, understandings, and feelings. In all of these cases, direct experience with other animals influenced people's perceptions and understanding about other animals.

The idea that humans learn through interacting with members of another species is a research area full of possibility. I find the idea of cross-species learning fascinating, and I was excited to explore whether educators who work at programs with live wolves learned things both about, and more interestingly for me, from the wolves themselves. I concur with Greenwood (2010) who argues that one part of "reinhabiting" place, that is, learning to live in a manner that avoids social and ecological harm, is to take the time to listen to the "teachers" (p. 22). He writes, "We need to learn how to privilege the teachers — other species, their languages....as full partners" (p. 22). As such, I suspected that wolf educators who interact directly with wolves have insights into what wolves have to tell or teach us. I had hoped, through examining the shared experiences of educators and wolves, to find a way to listen to, and learn from wolves.

While there are many wolf education programs with a conservation focus in North America and beyond (Fritts, et al., 2003; Troxell, et al., 2009), my thorough investigation of the literature only unearthed three studies that examined the value of wolf education (i.e., Black \& Rutberg, 2007; Samuelson, 2012; Willard, 2008). Despite a paucity of research evidence, those involved in wolf conservation continue to recommend education and public outreach (albeit often with caveats) as an important avenue to improving people's attitudes about wolves, and ultimately, human-wolf relations (Andersone \& Ozolins, 2004; Ericsson \& Heberlein, 2003; Fritts et al., 2003; Troxell et al., 2009). Investigating wolf education programs, including the intended messages, therefore 
seemed warranted. Given the current absence of research on wolf education, an in-depth study such as the one I outline in the chapters that follow, can enrich our understanding of certain aspects of the learning that goes on at some of these programs. Further, given there is so little research on wolf education, it made sense to first attempt to gain understanding of wolf educators' intentions and experiences prior to assessing what participants might be learning or evaluating the efficacy of particular programs.

A final rationale for my research arises from an ethical and moral standpoint: if humans accept the premise that wolves should have the right to exist, we need to figure out how to co-exist with them (Lynn, 2007). A change in human actions and attitudes will be needed to avoid further extirpations or even extinctions of large carnivores such as wolves (Ripple et al., 2014). Lynn (2002) discusses how a person's underlying morals and ethics drive beliefs about nature and human-nature relationships, including humanwolf ones. He suggests, and I agree, that the recovery of wolves is more about values than it is about facts. Indeed, Willard (2008), reflecting on wolf education and outreach efforts in the western United States, concluded that educators involved in controversial issues such as wolf management "must step beyond the comfort zone of science and rationality and acknowledge the values and social issues at the heart of the conflict" (p. 58).

I want to push this discussion a bit further here. I not only wanted to explore some of the ethical aspects of wolf education through the eyes of wolf educators, but also examine wolf education and human-wolf relations through a non-anthropocentric lens. Fawcett (2013) argues that environmental education research needs to better acknowledge the interdependences, "relationality" (p. 414), and value of nature and other 
species. Rather than the anthropocentric focus on nature as only important because of its ability to serve humans and human needs, she argues that "[h]olding on to our ecological embeddedness and advocating for environmental just conditions is a prime directive for environmental education research” (p. 415). My choice to investigate educators' experiences with specific wolves, thereby acknowledging that there indeed was an intersubjective relationship to explore, is one way I recognized the importance of nonhuman animals and our embeddedness as humans in a world with them. I will speak more on this matter later.

Education may play a role in helping people grapple with the ethical, social, and value-laden issue of wolf survival. To date, however, there is little research looking at whether this is actually taking place. We need to understand both what wolf educators are intending to communicate at wolf programs and how participants are responding. This dissertation offers a small window into understanding some of the ethical pieces from the educators' perspectives.

\section{Personal Stance}

Before I delve into the literature review and methods in the next two chapters, I want to address my personal position with regards to this research. I chose a study design (qualitative phenomenology with a critical lens) that aligned with my belief that no researcher can be truly neutral or objective. My own personal feelings and beliefs about education and about wolves led me to researching this subject in the first place, and also coloured how I ended up interpreting the experiences of the educators I interviewed and observed. Traditionally, a key idea within phenomenology was the importance of 
"bracketing," that is, setting aside one's own ideas about the phenomena in question (Lichtman, 2010). In her book about qualitative educational research, Lichtman (2010) reflects on her own experiences with phenomenology that led her to argue that "it is too simplistic to think that a researcher can set aside his or her own ideas about a phenomenon" (p. 80). She discusses, instead, the value in making explicit one's personal ideas about the phenomena under study before the research process begins. Following suit, I want to make explicit my ideas regarding wolf education and human-wolf relations.

Growing up in southern Ontario, I spent considerable time in my local rural outdoor environment exploring the woods, fields, ponds, and streams. This, along with family camping trips, fueled my passion for the flora and fauna of natural environments and from a young age I developed a deep concern for the health of other species besides humans. I never had direct experiences with wolves as there were none residing in my local region. Rather, my early exposure to wolves was through stories: European-based fairy tales and fables. I listened to stories such as Little Red Riding Hood, The Three Little Pigs, and Peter and the Wolf, so my first impressions of the wolf were likely that of villain. Still, I remember early on realising through my often-daily exposure to other wild animals (turtles, frogs, raccoons, skunks, foxes, deer, birds, etc.) that characters in books were rarely representative of the actual animals.

My interest in natural history and outdoor recreation flourished into my teen and adult years and I pursued outdoor/wilderness education as a career. While guiding trips in the Boundary Waters of Minnesota, in Quetico in Ontario, and on Lake Superior, I was often aware of resident wolves even though I rarely saw them outright. I remember 
hearing them howling to each other, coming across their tracks in the snow, or sometimes encountering a kill site (for example, a dead moose on a frozen lake surrounded by fresh tracks). Ely, Minnesota, the town near where I worked, was home to the International Wolf Centre, and I visited it several times and was impressed by their displays and programs.

My experiences travelling and teaching in wilderness areas have engendered in me certain beliefs and feelings about wolves. For one, I believe that wolves should have the right to survive and flourish. Ethically, I believe that individual non-human animals (such as individual wolves) should have certain rights based on their intrinsic worth. At a species level, I think the health of other species, while important in and of itself, is also important for the health of the planet and subsequently humans. Do I think wolves should never be managed, including in some cases killed? This is a harder question for me. To some extent, I sit on the fence on this matter given local contextual factors matter very much here. I do think some methods of management (e.g., sterilization, helicopter shooting, strychnine) are abusive and unethical.

As for wolf education, I do not believe good education is a matter of merely transmitting facts; neither do I believe any information is neutral and unbiased. I have come to this position in part through working for over a decade in outdoor experiential education where my focus has been on student-centred education comprised of concrete experiences and reflection, with the onus for learning being on the learner. I have both witnessed and experienced firsthand how our histories, cultures, and past experiences colour and affect what we both teach and learn. In my case, I care about wolves and want to see them and their habitats thrive. Having now interviewed 17 wolf educators, I have a 
clearer sense of what wolf education's role should or could be in helping make this happen, but I am by no means an expert on all aspects of wolf education. Nonetheless, ideally, I think education should expose people to new experiences, ideas, and perspectives, and allow them to come to their own conclusions, including how to act in accordance with their positions.

I knew when I began this research that many people may not share my positive feelings about wolves and desires for their survival, including the educators I intended to interview. I understood that my participants and/or those that attended the programs where they worked might have experiences, cultures, and histories that engendered in them a different set of beliefs. For example, perhaps their experiences included watching wolves tear apart a deer, a seemingly violent act. Or perhaps they grew up on a ranch or farm where their livelihood was impacted negatively by wolves. Maybe they lost a beloved pet to wolves. Any number of factors might influence what they felt and believed about wolves. My goal as a researcher was to explore, not contradict or judge, and I understood that my own ideas and beliefs might be quite different from those of my participants or the visitors that come to their centres and programs. I explain some of the ways I attempted to manage my own biases in the methods chapter.

Finally, I want to make clear that my shared experiences with other animals, including experiences with wild animals in outdoor settings but especially experiences with sled dogs and companion dogs, have influenced me significantly. As a young adult, living with my first companion dog, Ursa, for example, opened a window into my understanding of the depth of social and emotional worlds that animal-others experience. As just one example of how my relationship with Ursa influenced my actions, except on 
rare occasions, I have not been able to eat mammals since becoming her companion. As well, experiences working with, and oftentimes depending on, sled dogs enhanced my understanding about the depth, richness, and diversity of animal-others' social-emotional worlds. Working with groups of sled dogs and humans, I witnessed a multitude of sentient relations playing out, both amongst the dogs, and between dogs and humans. In part, these experiences led me towards caring more about animal welfare in general. Animal welfare is a term that has different definitions and meanings, however in my case, I mean the social movement that calls for better protection, respect and dignity for animal-others, hopefully driven by an attitude of kindness and compassion (Bekoff, 2010).

These personal experiences also fostered my curiosity about human-animal relations, and my desire to better understand shared interspecies experiences and possible emergent learnings. I wondered if other people too, have had experiences interacting with other animals powerful enough to shift their core beliefs and subsequent actions? Before setting out on this research, I suspected wolf educators' experiences interacting with wolves may have led to similar powerful learning; still, I knew that their experiences might also be unique, especially when considered within the context of the highly charged, value-laden conflict around wolves and wolf survival. Ultimately, I believe my dissertation research did not disappoint in that regard as will become clear in chapters five, six, and seven. 


\section{CHAPTER TWO \\ LITERATURE REVIEW}

\section{Introduction}

An examination of the peer-reviewed literature looking solely at wolf education would be brief. After extensive investigation, I uncovered only three studies that investigated wolf education (Black \& Rutberg, 2007; Samuelson, 2012; Willard, 2008) and only a handful of articles that mentioned wolf education in some capacity (e.g., Fritts et al., 2003; Switalski, Simmons, Duncan, Chavez, \& Schmidt (2002); Troxell et al., 2009). Nonetheless, research conducted in relevant areas such as environmental education, humane education, and human-wolf relations also provide insight into certain aspects of wolf educators' experiences along with an understanding of why public education around wolves is both recommended and employed as a means to conservation in the first place. For these reasons, the following review of literature will delve into an eclectic mix of primarily social science research and writing.

This literature review is structured to move from the broad and contextual to the specific. In the opening section I focus on wolves and human-wolf relations, arguing why wolves matter, then examine some historical, attitudinal, ethical, and cultural aspects of human-wolf relations. The human-wolf relations literature provides a background to understanding the circumstances and climate within which wolf educational efforts arose as well as factors that impact educators' experiences with wolves and wolf education currently. The final two sections narrow in scope: I first discuss relevant research from my home disciplines, environmental and humane education, that inform my 
understanding of wolf education and conclude by delving into research and scholarship that specifically focuses on wolf education.

\section{Importance of Wolves}

Whether wolves matter is a question that lies at the heart of their continued survival on the planet thus is well worth exploring. This question also relates to my research since many educational programs are founded on the premise that wolves do matter and are worth protecting (Troxell et al., 2009). The importance of wolves and wolf survival can be approached from different directions. In this section, I first tackle the question from a more anthropocentric perspective focusing on why wolf survival matters to humans. Included here are economic, social, and ecological rationales. Wolves' intrinsic right to exist is also well worth considering (Lynn, 2007) and is discussed in a section on ethics later in the review. Personally, I believe humans have a responsibility to help wolves recover, especially when we consider that many reduced and extinct populations are the result of massive wolf exterminations that took place at the hands of humans often employing cruel methods (Emel, 1995; Lopez, 1978). This too is described in more depth below.

The "cost" of wolves, such as the loss of livestock and pets when wolves prey on domestic species, is often the focus of media reports (Karlsson \& Sjöström, 2007). Less emphasized is that wolves frequently also have an economic benefit to humans (Ripple et al., 2014). Fritts, et al. (2003), for example, write that the International Wolf Centre in Ely, Minnesota brings in approximately three million dollars a year to the area because of the tourism it generates. Similarly, Chadwick (2010) states that tens of thousands of wolf 
ecotourists visit Yellowstone National Park each year, and Ripple et al. (2014) suggest that wolf ecotourism associated expenditures in Yellowstone generate anywhere from \$22 to \$48 million annually. The Ontario Ministry of Natural Resources (OMNR) (2005) also cites wolves' economic importance, albeit this is from dead wolves through the marketing of 600 pelts a year in Ontario. The OMNR also mentions their social importance, including their significance to some Aboriginal peoples: "The wolf symbolizes love and care for family and community, loyalty and co-operation" (p. 3). As well, for certain people, even if they never see a wolf, there is value "simply knowing that wolves exist in the wild" (Fritts et al., 2003, p. 299).

Ecologically, wolves are also important. Several ecological benefits of the wolf include: weeding out weak prey, controlling prey populations and therefore stimulating their productivity, and creating a food source for scavengers (OMNR, 2005; Ripple, 2014). In the northern Rockies region of the United States, predation by wolves reduces grazing by elk along streambeds and the increased vegetation allows for decreased erosion and thus better stabilization of stream banks, facilitating a return of aspen groves. As well, the carrion left by wolves feeds golden and bald eagles, coyotes, bears, magpies, and ravens (Chadwick, 2010). Eisenberg (2010), who has studied wolves much of her life, argues that the wolf is a keystone predator, that is, an animal that has an especially big effect on the health of ecosystems. She explains that various ecologists are finding that when keystone predators are removed from the ecosystem, major disruptions occur. She advocates for predators such as wolves to be seen as an essential part of healthy natural communities and summarizes the negative impacts of their removal succinctly:

Widespread issues created by predator removal include superabundant ungulates, biodiversity loss and development of plant and animal communities that differ 
markedly from what would occur naturally... [R]estoring keystone predators on an ecosystem scale can restore biodiversity via the many indirect effects they send rippling through food webs. This enhances bottom-up processes, such as nutrient cycling, and habitat for all sorts of species, including songbirds, butterflies, lizards, and fish. (pp. 190-191)

For all of the reasons outlined above (and their intrinsic value which will be discussed further below) surely it is worth considering the value of living in a world with wolves and what that might involve.

\section{Human-Wolf Relations}

Because it would be difficult to comprehend educator-wolf relations and some of the messaging in wolf education without putting these into context, I turn now to the literature on human-wolf relations. Particularly considering educational efforts arose in part to improve public attitudes towards wolves in the first place (Troxell et al., 2009), understanding these relations in more depth is worthwhile.

Fritts, et al. (2003) suggest that since wolves have had such a variety of relationships with humans, making generalizations becomes impossible. They also write that, "Persecution of the wolf has often been out of proportion to the threat it actually posed to people" (p. 289). For example, cases of wild wolf encounters with humans in Canada, Alaska, and northern Minnesota from 1900 through 2000 show very few examples of aggression (McNay, 2002). Looking at aggressive incidents, McNay (2002) found little evidence of unprovoked wolf attacks, with only one case documented between 1900 and 1969. While aggressive wolf-human encounters have increased since

\footnotetext{
${ }^{1}$ Despite APA's guidelines that block quotations be double-spaced, I chose to singlespace them for the sake of better readability and aesthetics.
} 
then, between 1969 and 2000 only 18 attacks were unprovoked (e.g., not self-defence, rabies) and of these, 11 of the wolves involved were habituated to humans.

Evidently, then, the often-negative history of human-wolf relations is more complex than the actual danger of wolves for humans. These relationships are frequently based on a history of conflict over territory and prey, concern over livestock, as well as influenced by cultural, social, and ethical factors. To show how complex and messy these relations are, Lynn (2010) examines social discourse on wolves in literature and demonstrates how wolves have multiple meanings and interpretations across people and societies. Some meanings of "wolf" include, "a teacher of hunting skills; an attentive parent; an exemplar of courage; a loyal pack member; a fellow creature... a fool driven by gluttony; a cowardly opportunist; a villain, varmint and vermin" (p. 82).

Human-wolf relations become even more difficult to unravel when wolves are not seen as wolves, but rather symbols for other things such wilderness or endangered species. While literature reviews like this one encourage summaries and generalizations, there is a danger in forgetting that both human and wolf communities consist of individual subjects with various characters and personalities thus making universal truths about interrelationships impossible. On that note, Lopez (1978) writes,

It can't be emphasized too strongly that the wolf simply goes about his business; and men [sic] select only those (few) things the wolf does that interest them to pay attention to... The mistake that is made here, with consistency, it seems, only by educated Western people, is to think that there is an ultimate wolf reality to be divined... (pp. 79-80)

Despite the challenges of overgeneralization and simplification, research that has been conducted on the subject of human-wolf relations does offer some interesting insights. 
In this section, I begin with a review of the history of human-caused wolf eradication followed by the present status of wolves. Next, I look at research considering human attitudes towards wolves followed by an examination of the influence of culture on human-wolf relations. This portion of this review concludes with a discussion of ethics.

\section{A Story of Eradication}

Sadly, part of the story of wolves and humans includes a history of massive, often organized efforts on the part of humans to eradicate them. At one time, the wolf had a more extensive range than any land mammal in the northern hemisphere; today this range is significantly reduced, partly due to extirpation attempts. Extermination of the wolf was most forceful and organized from the Middle Ages through the late 1800s (Boitani, 2003).

In the United States (not counting Alaska), the wolf was almost entirely extirpated due, in part, to negative beliefs and attitudes about the wolf on the part of settlers from Europe. Initiated in 1915, there was even a Division of Predator and Rodent Control (PARC), a part of the federal Bureau of Biological Survey, created with a "mission of eliminating wolves and other large predators from all federal lands" (Fritts, et al., 2003, p. 294). The project was a success. By the 1930s, in the contiguous United States they managed to purge the wolf from more than $95 \%$ of its former range (Morell, 2008). And by the 1970s, the only remaining wolves outside of Alaska existed in small pockets of wilderness in northern Minnesota and on Isle Royale, with possibly a few in northern Michigan (Boitini, 2003). 
In Canada, while wolves ultimately survived in more areas, they were still persecuted. Wolf bounties, for example, were introduced in Ontario in 1793, in Alberta in 1899, and in British Columbia in 1909. In the Northwest Territories, between 1951 and 1961 17, 500 wolves were killed as a predator control measure (Lopez, 1978). As a consequence, by 1900 they were completely wiped out in southern Ontario, southern Quebec, Nova Scotia, and New Brunswick. By 1930 they were also eliminated in Newfoundland and only a scarce few remained in other areas of Canada, for example, in Labrador, southern British Columbia, and southern Alberta (McNay, 2002). Further evidence of historic dislike for the wolf in Canada can be found in the fact that even in protected areas they held little respect. In the case of Algonquin Park, for instance, prior to 1958 rangers were encouraged to shoot wolves on sight (Theberge \& Theberge, 2004). In Lopez's (1978) oft-cited book Of Wolves and Men, he explores the history of wolf eradication especially in the United States, delving deeply into both the psychological and cultural reasons for this, searching for reasons why loathing and hatred for the wolf led to not just its elimination, but often depraved methods of torture and killing. He writes:

But the wolf is fundamentally different because the history of killing wolves shows far less restraint and far more perversity. A lot of people didn't just kill wolves; they tortured them. They set wolves on fire and tore their jaws out and cut their Achilles tendons and turned dogs loose on them. They poisoned them with strychnine, arsenic, and cyanide on such a scale that millions of other animals...were killed incidentally in the process. (p. 139)

I concur with Lopez that this widespread eradication and killing of the wolf is part of our shared interspecies history. Acknowledging that it took place, and attempting to understand why, will hopefully lead to improved human-wolf relations in the present and future. On that note, Lopez (1978) writes, 
It seems to me that somewhere in our history we should have attempted to answer to ourselves for all this...[T]he scope, the casual irresponsibility, and the cruelty of wolf killing is something else. I do not think it comes from some base, atavistic urge... I think it is that we simply do not understand our place in the universe and have not the courage to admit it. (p. 196)

\section{Status of Wolves Today}

Today, the situation for wolves has improved globally, but their status and level of protection depends on the country and region. For example, in Sweden, wolves are fully protected, while in Canada they are still hunted in open seasons (Boitani, 2003) and protected in only $2 \%$ of their land base (Theberge \& Theberge, 2004). The status of the wolf started turning around in the late 1960s and early 1970s with "strong and effective responses by governments" (Boitani, 2003, p. 321) helping the situation. For example, in the United States, wolves were listed as endangered in the Endangered Species Preservation Act in 1974, shifting the focus from earlier policies of eradication to conservation (Morell, 2008). A combination of factors may have led to increased protection including public education, protective legislation, urbanization (which influenced attitude change), as well as the voices of biologists who began learning about and sharing some of the more positive characteristics of wolves and their ecological importance with the general public (Boitani, 2003; Fritts et al., 2003; Kellert, Black, Rush, \& Bath, 1996; Troxell, et al., 2009). Prior to the legislative shift when the wolf became a protected species in the United States, some authors also note the important influence of Aldo Leopold, particularly the response to his famous essay "Thinking Like a Mountain" (1949; see Jickling \& Parquet, 2005; Kellert et al., 1996; Ripple \& Beschta, 2005). Leopold was involved in government sponsored wolf eradication. Following an experience when he was part of a group that shot a female wolf and he watched her die, 
he wrote, "I was young then, and full of trigger-itch; I thought that because fewer wolves meant more deer, that no wolves would mean hunters' paradise. But after seeing the green fire die, I sensed that neither the wolf nor the mountain agreed with such a view" (1949, p. 130). This experience precipitated a change in his own views, and he went on to become an advocate for protecting wolves as vital members of the ecosystem in the 1930s and 1940s (Ripple \& Beschta, 2005).

Despite these shifts in protection over the century, according to the World Conservation Union, at present the wolf remains "vulnerable" on a global scale. Boitani (2003), a well-known wolf biologist and researcher, believes that the greatest threat to the wolf now is habitat and habitat destruction. He also notes the importance of factors such as their dependence on positive human attitudes and consequent efforts for conservation. Ultimately, he explains, continued recovery will depend on a combination of factors: genetic, economic, biological, ethical, and cultural.

Across the globe, then, wolves are doing better than they were a century ago (Boitani, 2003). It will be interesting so see how populations change in the coming years and how these are associated with changes in human culture. Perhaps changing values towards nature in general will lead to appreciation and protection of wolves, creating continued avenues for recovery? For example, in Europe, since the 1980s smaller isolated populations of wolves have managed partial recovery due to agricultural and cultural changes such as changing beliefs about nature (Boitani, 2003; Chapron et al., 2014). Alternatively, will growing wolf populations lead to increased conflict? This seems to have already happened in the Rocky Mountain region of the United States. In some of these states, wolves were removed from the endangered species list as of April 
2009 (Bruskotter, Toman, Enzler, \& Schmidt, 2010). This decision caused an uproar.

Fourteen animal protection and environmental organizations waged a lawsuit against the federal government unless wolves in the western United States were relisted as endangered and a plan for conservation was developed that included protected areas and buffer zones where wolves can exist in natural packs without fear of persecution (Chadwick, 2010). Clearly, the road to continued wolf recovery will be a difficult one.

\section{Human Attitudes Towards Wolves}

Social scientists have conducted research trying to understand the intricacies of human attitudes towards wolves. The wolf educators I interviewed each had their own attitudes towards wolves as well as encountered a variety of attitudes from participants who visited the centres or programs where they worked. Surveying some of the attitude research helps elucidate some of the factors possibly influencing what educators learned through working with wolves. As a case in point, Anderson, Hill, Ryon, and Fentress (1995) discovered that when university students watched video clips of wolves interacting, especially longer interactions, participant's attitudes towards wolves influenced how they interpreted those interactions; simply put, if they liked wolves, the interactions were deemed friendlier.

Human attitudes towards wolves impact public support for, and therefore success of, wolf conservation projects (Boitani, 2003), which is likely why there are so many studies looking at attitudes towards wolves. Bruskotter, Schmidt, and Teel (2007) explain how these attitude studies can be an important part of gauging public sentiments around wolves, as managers and biologists may frequently hear the opinions of those who 
feel strongest about wolf-related issues, leading them to erroneously conclude that attitudes are more polarized than they actually are.

Both the research literature and contemporary news articles suggest that wolf survival, reintroduction, and recovery can be a polarizing issue with attitudes on both ends of the spectrum. For instance, in the United States west, Chadwick (2010) explains how some elk hunters despise the wolf and they "are depicted as four-legged killing machines_-land piranhas_-ravaging game populations" (p. 39). Similarly, posters created by anti-wolf groups describe them as "The Saddam Hussein of the Animal World" (Morell, 2008, p. 891). On the other side, there is ample evidence of positive attitudes and passion about wolves. For example, Fritts et al. (2003) point to an estimated 40 NGOs in North America and at least 12 in Europe that advocate for wolf conservation.

Understanding human attitudes towards wolves is thus a complicated endeavour. Sometimes the conflict is not really about the wolves themselves, but a platform for larger issues such as rural-urban conflicts or "over how federal land is used—an issue that often pits local and regional views against national perspectives" (Fritts, et al., 2003, p. 296).

In the section that follows, I provide an overview of some of the recent literature in this area including studies that: offer attitudinal overviews, investigate attitudes towards reintroduced wolves, consider social and cultural aspects of attitudes, explore attitudes in relation to proximity to wolves, and examine attitudes towards wolf management. While some conclusions about human attitudes towards wolves can be made, not all studies looking at attitudes take the same approach or obtain the same findings, making generalizations difficult. This could be due to changing attitudes over 
time or the country or region where the study took place. To begin, I look first at the big picture, that is, studies or literature reviews that provide overviews.

Overviews. In a literature review looking at human culture and large carnivore conservation in North America, Kellert et al. (1996) found that, in general, people who had higher levels of education, were urban, younger, and/or were members of environmental groups had more positive attitudes towards the wolf (such as believing wolves have ecologic, recreational, and existence value) while older, rural, and agricultural people (particularly farmers and ranchers) tended to hold more negative attitudes about wolves. Exceptions to the rule included hunters and trappers in the midwest of the United States and residents of one area of Manitoba who were pro-wolf conservation on the whole.

Later, Williams, Ericsson, and Heberlein (2002) attempted to summarize quantitative studies conducted from 1972 through 2000 on human attitudes towards wolf reintroduction. They chose 37 quantitative papers from three continents (North America, Europe, Asia) and generalized the surveyed data. On the whole, they found that the general public had positive attitudes towards wolves (51\%) and 60\% supported reintroduction at some level. Positive attitudes (meaning participants either liked the wolf or felt positively about wolf reintroduction) correlated with living in urban areas, higher levels of education, and membership in environmental groups. As in Kellert et al.’s (1996) review, negative attitudes correlated with both those who had greater potential for direct experience with wolves (farmers, ranchers, rural citizens) as well as being older, male, or Scandinavian. 
Houston, Bruskotter, and Fan (2010) also attempted a broad look at attitudes towards wolves in the United States and Canada. They tackled the project in a unique manner that resulted in findings that contradict Williams et al. (2002), making their study particularly worthy of mention. They examined attitudes in the context of print news media from 1999 through 2008 (newspapers and newswires). They classified articles as either positive or negative and as focused on attitudes, beliefs, or judgments about wolves. They then noted the location of the stories, grouping them into four designations: states or provinces where wolves had recently been introduced, had permanent populations, had no wolf populations, or recovery zones that lacked viable populations. Unlike Williams et al. (2002), they found that attitudes towards wolves were predominately negative. The most common belief was "that wolves negatively impact human activities (30.5\%)" and the most common judgment was "wolves should be killed or controlled (27.9\%)" and these "accounted for the majority of all expressions of the 10-year time period" (p. 397, emphasis in original). The most negative discourse came from states where wolves had been recently introduced and states with recovery zones where there was potential for reintroduction.

Houston et al. (2010) suggest, then, that the potential for wolf reintroduction might also be influencing public opinion. Interestingly, in states and provinces that have always maintained a wolf population, attitudes were less negative than areas of possible recovery or newly introduced wolves, leading them to conclude that "negative media attention and the heightened perceptions of risk should be reduced over time, as people learn once again how to live with wolves" (p. 401). It is worth noting that these researchers were looking at news stories, not directly studying people's attitudes, which 
while perhaps reflecting public attitudes, also might offer an over-sensationalized version of the issues.

Reintroduced and returning wolves. Houston et al. (2010)'s finding that attitudes tend to be more negative in areas where wolves have been absent seems to be supported when you consider the following three studies that investigated the public's attitudes around wolf reintroduction.

For example, Lohr et al. (1996) looked at public attitudes towards the reintroduction of wolves by surveying hunting and naturalist groups in New Brunswick, and found that while members of naturalist groups had more positive attitudes towards wolves than hunters, overall, none of the groups were keen about the idea of wolf reintroduction. The main concern seemed to be over the possible impact on deer and moose populations. Concerns over the deer population were also significant to participants in Enck and Brown's (2002) survey of New York residents regarding the reintroduction of wolves in Adirondack State Park. For these residents, concerns in order of importance were: possible restrictions by government on private land use, wolves impacting the deer population, wolves preying on pets, humans killing wolves, or wolves killing livestock.

When it came to the issue of reintroduction, those living in Adirondack State Park were almost equally divided on the issue while those in greater New York state were more positively inclined to the idea (60.2\%). Interestingly, Enck and Brown (2002) suggest knowledge about wolves and their potential impact in an area will not necessarily lead to more positive attitudes, as residents have varying attitudes about the same impacts (e.g., whether a reduction by wolves of local deer is negative or positive). They suggest 
that managers wishing to restore wolves might look at both the influence of media as well as the possibility of working together with stakeholders and residents on developing comanagement strategies.

For those living in Adirondack State Park, one main concern was the possible restrictions by government on private land use (Enck \& Brown, 2002). This concern was echoed by the participants in Scarce's (1998) investigation of the social aspects of wolf reintroduction in a town that borders Yellowstone Park in the United States. Scarce interviewed 40 residents and discovered that issues of power emerged; wolves were imbued with a variety of meanings and these meanings related to larger issues of control/power and self-determination/freedom. Many residents felt that science was used by the government as a tool of power over and above the knowledge and opinions of the local people. Wolves were seen as just another agent in this limiting of "residents' selfdetermination by controlling their lifestyles and their livelihoods" (p. 39) by for example, restricting their hunting or cattle grazing.

Sociocultural. For the residents in Scarce's (1998) study, wolves and wolf reintroduction were complicated issues bound up with social and political factors. The following four studies intentionally focussed on sociocultural influences on attitudes towards wolves. In all four studies, the authors suggest their research illustrates the pitfalls of simplified understandings of attitudes. For example, Skogen and Thrane (2008) conducted a quantitative study in Norway and found that those who supported a reduction in the wolf population were typically less educated, more traditional politically (i.e., tended to be opposed to modernity and support traditional rural activities and values), had less cultural capital (i.e., familiarity with and access to dominant culture), 
and trusted local or informal over institutional information. Ideas about an ideal wolf population size were intertwined with participants' more general "value orientation" (p. 31 ), which in turn was related to cultural aspects of social class.

These findings regarding social class and value orientation had similarities to an earlier study of Skogen's (2001) where he interviewed, individually and in focus groups, young people from eastern Norway in an area where wolves reside. He considered the effect of modernization and culture on attitudes about wolves and found that a subculture within the rural participants emerged that was comprised of working class males who pursued more traditional outdoor activities such as hunting and fishing. These youth, while not necessarily anti-wolf, were strongly opposed to current management of carnivores and felt that biologists and environmentalists wield too much power. In this study, there was a clear divide between those who trusted scientific knowledge that is abstract versus lay knowledge "gathered through concrete experience" (p. 219). Skogen critiques the hegemonic position that scientific knowledge holds over other forms of valuable knowledge, specifically that of working class people who interact with nature directly through resource extraction industries and outdoor recreation. He submits that managers, scientists, and a growing middle class often base their environmental values on scientific information and popular media. He concluded that lay knowledge can also contribute to our understanding about sustainability, and consequently, wolf-human relationships.

In another study, Skogen, Mauz, and Krange (2008) examined the narratives of anti-wolf advocates and those who were pro-wolf reintroduction both in France and Norway, honing in again on attitudes as they relate to class and culture. Like the other 
studies conducted by Skogen and his colleagues, the pro-environmentalists were a group predominately "within the middle class that are highly educated; employed in 'nonproductive' sectors; and have incomes in the medium range" (p. 127). It was often the anti-wolf proponents who were rural, working-class, and had limited access to power, and the authors suspect their narratives could be understood as a form of resistance against hegemonic power. As with Scarce's (1998) interviewees from the Yellowstone Park area, Skogen et al. (2008) concluded that issues around wolf reintroduction might have as much to do with power relations as they do about wolves. They propose that by "incriminating state services and scientific institutions" in their narratives, "wolf opponents launch resistance against the power of the state and its associates" (p. 123). Skogen et al. explain how both sides had narratives or stories they told to reinforce and explain their positions. In the case of pro-wolf advocates, the natural return of wolves maintains the story of the "victorious victim" where wolves have returned despite the odds. On the other side, anti-wolf proponents often told narratives of secret conspiracies of wolf reintroduction.

Finally, focusing specifically on cultural influences on attitudes in North America, Shelley, Treves, and Naughton (2014) conducted a survey to compare the attitudes of members of an Ojibwe band (the Bad River band of the Lake Superior Tribe of Chippewa) to other Wisconsin residents who lived in wolf territory. On average, the Ojibwe members felt more positively towards wolves and wolf protection and were less supportive of a wolf harvest. The authors point to how, for the Ojibwe, the wolf plays a key role in their cultural stories and how this likely influences their attitudes about wolves and wolf management: "Wolves are often referred to as brothers and sisters with a 
perception, or prediction, that what happens to the wolf will also happen to the Ojibwe" (p. 409).

Distance from and/or experience with wolves. Some attitude studies have been conducted to look at how distance from wolf populations and territory influences attitudes. Ericsson and Heberlein (2003) and Karlsson and Sjostrom (2007) both conducted survey studies in Sweden that examined the impacts of where people lived in relation to wolf habitat on their attitudes. Both studies found that the further from the wolf territory the better the attitudes towards wolves. Ericsson and Heberlein (2003) investigated if direct experience with wolves could be one explaining factor, and while they discovered that experience with wolf predation can lead to more negative attitudes towards wolves, they suggest that negative perceptions and feelings about wolves were not necessarily due to direct contact. Rather what wolves seemed to represent mattered most: "Wolf restoration to minority groups like hunters and people who live in wolf areas may symbolize the dominance of the urban society over rural values. Wolves become just another way that the core area of a society exerts dominance over the periphery" (p. 156). Karlsson and Sjostrom (2007) too found that negative attitudes were not necessarily related to direct experience with wolves since many participants in their study had no direct experience. Instead, they also propose indirect experience with the species matters. For example, those in wolf areas are more exposed to negative media as well as secondary accounts from fellow hunters and/or farmers and ranchers. As the authors state, "Bad experiences are usually much more communicated than good ones" (p. 614). Røskaft, Bjerke, Kaltenborn, and Linnell (2003) investigated the attitudes of Norwegian residents towards four large carnivores (bears, wolves, lynx, and wolverines) 
through surveys using a large sample (depending on the question, 1304-2921

respondents). They found that those living in areas where the carnivore species roamed reported less fear than those living in areas without them. They also found a relationship between outdoor activities and fear. For example, when it came to wolves, there was a relationship between the likeliness of being "not afraid" and taking part in outdoor activities such as small game hunting, mountain hiking, and berry picking. They concluded that "both fear and other aspects of negative attitudes towards large carnivores will be reduced with exposure to them; ... people tend to be less afraid and have more positive attitudes towards carnivores the longer carnivores have been present in the area" (p. 194).

Finally, in southwestern Alberta, Sponarski, Semeniuk, Glikman, Bath, and Musiani (2013) investigated residents' attitudes towards wolves, honing in specifically on rural residents. They found that attitudes were diverse and heterogeneous and concluded that it is dangerous to make generalizations about the attitudes of rural residents. They suggest that wildlife managers may need to incorporate a variety of communication strategies depending on which rural group is being targeted.

Public attitudes towards management. I turn now from studies of attitudes towards wolves themselves to research on public opinions and attitudes about the management of wolves. In Finland, Bisi et al. (2007) conducted a qualitative study of the creation of a wolf management plan and Naughton-Treves, Grossberg, and Treves (2003) looked at public opinions of wolf management through a survey of 535 Wisconsin rural residents. While the details of these two studies are not necessarily important to understanding aspects of wolf education, there were some findings that are worth noting. 
For example, both studies concluded that human-wolf conflict is complex and valuebased; simple solutions to this conflict thus do not exist.

In Wisconsin, compensation payments for lost pets and livestock is one management strategy, but these payments do not necessarily improve tolerance levels (Naughton-Treves et al, 2003). Survey respondents felt that the payments were inadequate (partly due to the emotional toll of losing an animal), did not compensate for the suffering of their livestock, and did not cover all their losses because Wisconsin requires proof of wolf predation for compensation and according to respondents that was not always possible. Adding to the complexity, Naughton-Treves et al. also found that while the loss of domestic animals influenced tolerance, more significant was a respondent's identity as a bear hunter, livestock owner, or rural resident.

Management strategies such as compensation are demonstrably complex. In the face of this, Bisi et al (2007) and others (Bath, 2009; Todd, 2002) have concluded that involving the public in decision-making processes may be one way of mitigating some degree of conflict. This idea is also echoed in a paper by Nie (2002) that explored the value-based nature of wolf recovery and management; he writes, "it is imperative that the wolf decision-making process be as inclusive, participatory and representative as possible...Multiple stakeholders with multiple values must be given a larger role to play" (p. 70).

Conclusion. To summarize this section, there are a range of studies looking at human attitudes towards wolves, wolf conservation, and wolf management, and the findings are not consistent. What becomes clear in reviewing these studies is that human attitudes towards wolves involve a mess of multifarious factors that may include: gender, 
culture, class, age, level of education, rural/urban, group membership (e.g., hunter, naturalist, environmental), region, country, political beliefs, distance from wolf territory, attitudes towards nature generally, relationship to nature and the land, values, issues of power and control, and trust in government authority and/or science. Overall, human attitudes in recent years appear to have improved as evidenced by the fact that countries are no longer employing massive extermination programs and the wolf has recovered, returned, or been reintroduced in places. Generally, attitudes, as shown in the larger studies seem to indicate more people feeling positive or at least neutral towards wolves. I believe one of the key insights to draw from reviewing research on attitudes is the importance of context, that is, understanding that wolf attitudes and values are often specific to a particular place, culture, and a certain time in history.

\section{Influence of Culture}

As noted in the research reviewed above, culture was often a factor that influenced attitudes towards wolves. Some authors have explicitly honed in on how culture can affect perception and subsequent treatment of wolves. Fritts et al. (2003) argue that cultural and social influences play an important role in the perception of the wolf. For example, historically, many North American Indigenous cultures had great respect for the wolf and "often regarded them as spiritually powerful and intelligent animals" (p. 291), in Greece and Rome a positive view of the wolf has been in place for several centuries, whereas in northern Europe, there has been more negativity. Human cultures in each of these regions have interacted with the wolf throughout history, but differing attitudes and beliefs have emerged (Boitani, 1995; Fritts et al., 2003). 
Boitani (1995) has comprehensively examined culture's influence on the perception of wolves and offers some theories to explain these drastically differing attitudes. He suggests that historically, different cultural relationships between humans and wolves arose depending on the predominant means of subsistence, for example, whether people were primarily hunters and warriors, farmers, nomadic shepherds, or sedentary shepherds. He goes on to explain that if a particular culture subsided mainly through war and hunting, their overall view of the wolf was positive and the wolf was even emulated. For instance, the wolf appears often as a character with high status and respect in the early stories and legends of hunting cultures. In contrast, nomadic shepherding cultures often had negative attitudes towards wolves because people would have had to defend their livestock and livelihoods from predation. Sedentary shepherding and farming peoples were less negative towards wolves with a "tense, but relatively peaceful coexistence" (p. 8). For farmers, the wolf was an unavoidable nuisance, but only one of many that had to be dealt with. Boitani hypothesizes that historic relationships with wolves in Europe and Eurasia still influence present-day attitudes both there and in North America where settlers brought their preconceived attitudes with them. He explains, for example, that many early North American immigrants were from northern and central Europe, areas with a history of nomadic shepherding and, subsequently, very negative attitudes and beliefs about wolves. These early immigrants considered North America a vast wilderness - one that needed to be subdued and "[w]olves and Indians [sic] became symbols of the hostile environment they were trying to conquer" (p. 9). 
Cultural attitudes towards the wolf can also be gleaned from a group's stories, fables, and legends. Different culture's stories characterise the wolf differently, for example, "the wolf is wise (Irish folktale), the wolf is ferocious (a Pennsylvania legend), a wolf is foolish (German folktale), a wolf is friendly (Japanese folktale)" (Nie, 2002, p. 66). Lopez (1978) notes how in some western cultures, "It is Aesop's wolf, not Science's wolf - a base, not very intelligent creature, of ravenous appetite, gullible, impudent, and morally corrupt — that generations of schoolchildren are most familiar with" (p. 251). Raised on stories and fables where the wolf is depicted as an evil, corrupt villain, like in the stories of Aesop and Grimm, he writes that, "Some children weaned on fable never inquired deeper into the animals than the stories led them, and so went through life believing the wolf evil, the fox sly, and the bee industrious, and the ass foolish" ( p. 251).

In a similar vein, Schlickeisen (2001) writes about the cultural barriers to wolf reintroduction in Adirondack State Park, pointing to the role of negative symbolism of the wolf in story, fable, and legend. He writes that stories such as "Little Red Riding Hood" and "The Three Little Pigs" along with Aesop's fables are good illustrations of the "powerfully negative symbolism" (p. 61) and prejudice towards wolves that came with European settlers to North America.

Negative representations of the wolf in story and legend both reflect and reinforce cultural beliefs. Religion, too, can be a window into cultural attitudes. For example, the wolf in Europe in the Middle Ages appeared often as a representation of evil in legends about the Catholic saints (Frits et al., 2003). Similarly, Lopez (1978) believes that wolf eradication by settlers to North America represented certain Christain beliefs of the time: 
"As civilized man [sic] matured and came to clearing trees for farms and clearing pagan minds for Christian ideas — the act of killing wolves became a symbolic act” (p. 141). Antonio (1995), too notes how wolf killing in the United States reflected religious beliefs, asserting,

So much of our atavistic memory of wolves seems to be dominated by this essentially religious concept of the devouring demon, augmented in legend by a Grimm romanticism of evil...For generations of our ancestors, the werewolf, not to mention the she-wolf, became the personification of pure evil. (p. 220)

However, it is important to note that not all religious representations have endorsed the wolf as a symbol of evil. For example, in Japan prior to 1868, the word wolf or ookami could be translated into "great god" (Frits et al, 2003, p. 293). Farmers, especially, thought of wolves as allies because they killed crop-destroying wildlife. In fact, in the 1600s, wolves in Japan were prayed to in shrines (Frits et al., 2003).

On a different note, Midgely (2001) discusses the idea that for some cultures, wolves represent human vices. She writes:

To speak of people as wolves, rats, vipers, sharks, or vultures is not just to say that they are troublesome. It is to accuse them directly of vice. And among these vice-demoting animals the most vice-denoting of all in our tradition has been the wolf, as one can check by looking up the entries under wolf in any quotation dictionary. (p. 181)

She believes that projecting human vices onto animals appeases the human conscience for killing them. But she also believes such projection serves another purpose: that killing the animals becomes representative of killing human vices.

Thomas (2001), an Onandaga of the Wolf Clan, also discusses the often-negative cultural attitudes settlers brought to North America and contrasts them with an Aboriginal perspective of wolves. Prior to European colonization of the Adirondack State Park area, 
he explains how his people respected wolves. In contrast, he describes insights gleaned from reading the diaries from early European immigrants:

[T] hese people were afraid to sleep at night for fear of the wolf's howl. The diaries said that the people stood with clubs behind their backs, and when the wolves came down the path, they used these clubs to kill them. Our oral tradition tells us that before electricity and roads, our people, the Wolf Clan of the Onandaga, as brother and sisters of the wolf, could walk down a path past wolves without fear. We had respect, we were related, and the wolves and my ancestors shared trust. When the wolf walked down the path, we would step aside and let it pass. We respect the natural world and all living things. Respect is essential to understanding. We understood. The settlers did not understand, for they were fearful in their hearts. (p. 48)

Culture obviously has a big role to play in how wolves are understood and

ultimately treated. Whether through the sharing of stories and fables, religious and moral education, or membership in a clan, people are encultured into a way of thinking about wolves. As evident from the discussion above, it can be one that emphasizes respect or one dominated by fear and hatred. The latter obviously can lead to people being unsupportive of wolf recovery and conservation.

Culture, then, can influence people's beliefs and feelings about wolves. While all 17 of the educators I interviewed worked at programs in North America, and consequently were educating in a western cultural framework for the most part, cultural influences and contexts did emerge as key in my findings as will be seen in chapters five through eight.

\section{Ethics}

Eisenberg (2014) writes, "But beyond collaboration, the other necessary ingredient for coexisting with large carnivores (or with any living beings) has to do with ethics - how we see our relationship with the environment" (p. 244). Both wolf education and wolf conservation have ethical aspects worth exploring. For example Lynn 
(2002) suggests that, "wolves persisted quite well alongside humanity for over a hundred thousand years, all without the 'benefit' of wildlife management...Resolving this conflict is a question of values, not facts" (p. 313). He asks, what would it mean, ethically, for humans to live alongside wolves? For him, "a deep recovery of wolves is interlaced with the recovery of our animality, which is to say, our human identity as animals in relationship to other animals" (p. 319).

In a later article, Lynn (2010) argues that ethical discourses are given the least credence when it comes to discussions about wolves while scientific ones have been privileged. Nevertheless, recently more discussion of the ethical dimensions of wolf conservation have begun to emerge. Lynn believes ethics can play an important role in human-wolf interaction, policy, and management. Whatever the discourse, whether social, ecological or ethical, he reminds us to see all approaches as incapable of neutrality: "whether our theories are hunches, testable propositions, or expansive worldviews, they are always fallible, contestable, and in the making" (p. 85). He uses the metaphor of each discourse (social, ecological, ethical) looking at issues from a different mountaintop. Together, each "view" can add to the creation of a more expansive and complete vision of the best way forward.

Jickling (2005a) and Jickling and Paquet (2005) probe the ethics and philosophical underpinnings of science when it comes to wolves and human-wolf relations. Jickling (2005a) uses the example of wildlife management of wolves in the Aishihik region of the Yukon as an example. He questions the underlying assumptions in the science of wildlife management and argues that wolf killing in the Yukon was "dressed with the 'respectability' of science and the 'objectivity' of experimental design" 
(p. 39). Like Lynn (2010), he argues that more focus should be placed on the ethics and value dimensions of issues because when ethical or "right relationships" are considered, then the acts that follow are more mindful. Jickling states that, "When we are mindful and respectful then we act with courtesy and etiquette, including trans-human etiquette" (p. 41). In a similar vein, Jickling and Parquet (2005) worry that some of the sciencebased practices of wolf management that are currently accepted "are often taken for granted, transparent, and uncontested" (p. 118).

Fox and Bekoff (2009) also explore some of the underlying ethical issues around wolf recovery and management. For example, they question definitions of wolf recovery success in the United States "when we have confined recovery efforts to less than 5 per cent of the wolf's historical range and when approximately 80 per cent of all known wolf mortalities in the tri-state area of Montana, Idaho, and Wyoming are intentional removals by the U.S. federal government?" (p. 121). They wonder how humane it is to work to recover wolves just to turn around and kill them later as a management strategy? They argue that decisions about how wild wolves are treated have not considered the wolves' perspectives. They suggest, then, that research that has shown that animals such as wolves have rich emotional lives should be taken into account when making decisions about how wolves are treated and "managed". Fox and Bekoff outline some principles that might guide the ethical treatment of other animals like wolves including, "do no intentional harm, treat all individuals with respect and compassion, and recognize that all animals have intrinsic value or worth, irrespective of their utility to other animals, including humans" (p. 121). 
Both Antonio (1995) and Emel (1995) explore ethics around wolves through feminist lenses. Antonio discusses the possibilities of reinventing human-wolf relationships through recognizing the wolf as a species that shares both similarities and differences with humans and argues for the need to develop a "deeper ethical relationship to difference" (p. 226) that looks beyond wolves' alleged rapacity. She argues that it is important to move beyond the wolf as a representation of evil to it being respected for its positive qualities, some of which it shares with us:

...its fierce maternity;... strength in solitude; sensitivity to small but important changes in the environment, emotional or physical; intelligence, sensuality, playfulness, resilience, and powerful hunting instincts (one may hunt for truth or value in life, as well in physical nourishment); and ... enjoyment of freedom and sensory richness. (p. 228)

Emel (1995) takes an ecofeminist approach in her discussion of wolf eradication in the United States, pointing to the similarities in motivation and timing in how both Native Americans and wolves were treated by those in power: "Like the Native American, the wolf was killed to secure land and investment" (p. 720). The wolf, she explains, was also "killed to sustain big game animals so that human hunters could kill them. It was killed for pelts, for data, for science, and for trophies. It was tortured, set on fire, annihilated" (p. 720). Like Antonio, Emel (1995) challenges stereotypes of the wolf as a ruthless killer, especially when perpetuated by many of the same people who were responsible for the massive unconstrained slaughter of buffalo. She also describes how killing can and has informed constructions of male identity and masculinity. She stresses the importance of probing such human constructions of identity because of the implications they can have for other animals such as wolves. She writes, "To leave unexamined the structures, be they gender, race, class, or culture, that teach us to slice off 
or repress empathy and to distance ourselves from the 'other', invites oppression, brutality, holocaust" (p. 731).

When it comes to wolves, why have ethical discussions such as those described above been mostly overlooked? One possibility is the historical framing of other species in western cultures. Corman (2011), writing about the educational implications of Freire's views of other animals which very much reflect western perspectives in general, notes that, "nonhuman animals are never individuals; instead they are defined strictly in terms of species membership: Variability among individuals and group-specific differences among populations within species are flattened" (p. 35). Further, nonhuman animals are objectified and "[s]tripped of capacities to dialogue, communicate, possess consciousness, transform, and actively transcend their prescribed natural destiny" (Corman, 2011, p. 38). Given this context, it perhaps is no surprise that the ethics of wolf eradication and killing, conservation, and treatment have seldom surfaced. Research that moves beyond investigating human-wolf relations on a species level, but rather considers both the learning that can come through interacting with individual members of another species (such as educator-wolf relations) is timely. When other animals are considered and researched as subjects, it may help move ethical discourse around human-wolf relations closer to the fore.

Moving now from literature specifically related to human-wolf relations, I turn now to relevant writing from the fields of environmental and humane education. 


\section{Environmental and Humane Education}

While there is little research examining the experiences of wolf educators or wolf education itself, there has been research conducted that is relevant to wildlife educators and wildlife education more generally. This research falls within the domain of environmental education. Environmental education can be loosely defined as an interdisciplinary field of education focused on "the nature of people-society-environment relationships" (Stevenson et al., 2013, p. 2) in both informal and formal settings, and ranging in orientation from the local to the global. It has concentrated on our attitudes, values, knowledge, and understating of environmental issues and concerns as well as "developing the agency of learners in participating and taking action" (Stevenson et al., 2013, p. 2).

Similarly, in the neighbouring field of humane education, a growing number of researchers are conducting research that considers human relationships with other animals. Contemporary humane education is defined by Humes (2008) as a form of education that "attempts a broad analysis of injustice by examining relationships between humans, other species, and the earth, [encouraging] caring, compassion, empathy, kindness, non-violence, respect, responsibility, and sensitivity to others" (p. 68). Below I discuss some historical and philosophical underpinnings of both these educations, followed by descriptions and discussion of selected relevant research.

\section{Environmental Education Overview}

In the face of warnings about environmental degradation of catastrophic proportions facing the planet and the dire consequences of pollution, climate change, deforestation, overfishing, overpopulation, and mass consumption for both humans and 
other living species with whom we share our home (Kahn, 2009; Orr, 1994), critical environmental educators demand a complex response that pays attention to not only the natural but also the social, cultural, and political dimensions of these problems (e.g: Castellano, Quirino de Luca, \& Sorrentino, 2011; Fawcett, 2013; Humes, 2008; Kahn, 2009). For example, many environmental education researchers point out that often the most polluted and toxic environments are inhabited by the poorest and most marginalized in society (Bell \& Russell, 2000; Haluza-DeLay, 2013; Kahn, 2009). Thus, there is a growing understanding that social concerns are intertwined with environmental ones and the two are not easily separated (Kahn, 2009).

This emerging critical stance taken by many environmental educators and researchers today is in stark contrast to the dominant discourse in the early days of the discipline. Depending on who is authouring the overview/history of environmental education, different versions of the story will emerge. As Russell and Fawcett (2013) state, histories "depend very much on who has the privilege of telling the tale" (p. 369). I find Gough's (2013) recent historical account of the field from the recently published International Handbook of Research on Environmental Education particularly helpful, so I draw particularly from it, along with a few other sources, in the brief overview that follows.

Environmental education has a variety of roots including rural and nature study, and conservation education (Palmer, 1998). Gough (2013), along with others (Hart \& Nolan, 1999; Palmer, 1998), discuss how environmental education probably emerged as a field in its own right during the 1970s, with the 1977 international governmental Tbilisi UNESCO-UNEPA conference having a significant influence on the development of the 
field. In part, it arose out of the need to raise public awareness of the ecological problems highlighted by scientists during the 1960s such as air and water pollution, overpopulation, and resource depletion (Gough, 2013). Perhaps due to its scientific roots, environmental education research in the early years was conducted almost exclusively from a positivistic scientific paradigm that favoured quantitative approaches (Gough, 2013). Indeed, Hart and Nolan (1999) in their extensive literature review of environmental education research found that $90 \%$ of research in the 1970 s was done employing quantitative methods. These positivist inquiries, focussed mostly on changing environmental behaviour, dominated the field up until the 1990s (Gough, 2013).

Kollmuss and Agyeman (2002) discuss how the positivist approaches that focused on behaviour were lacking because the underlying assumption that increases in knowledge and awareness necessarily leads to pro-environmental behaviour was overly linear and thus simplistic. In their oft-cited paper, they explore a variety of paradigms and factors that may explain the gap between environmental knowledge and proenvironmental behaviour and conclude that " $[\mathrm{m}]$ any conflicting and competing factors shape our decisions" (p. 256), with knowledge being just one of these. They suggest that:

environmental knowledge, values, and attitudes, together with emotional involvement [make] up a complex we call 'pro-environmental consciousness'. This complex in turn is embedded in broader personal values and shaped by personality traits and other internal as well as external factors. (p. 256, italics in original)

Due in part to the deficiencies of overly simplistic approaches, since the 1990s, environmental education research has expanded and a greater variety of approaches have been taken, including interpretivist, critical, postmodern, poststructuralist, postcolonial, feminist, and socioecological (Gough, 2013). This would please Hart and Nolan who in 
1999 argued that "the dominant worldview is not the only view of reality or way of knowing and ... we need to explore multiple frames of reference to more fully understand human-environment relationships" (pp. 32-33).

Demonstrating how rich and diverse environmental education and research has since become, Sauvé (2005) outlines 15 pedagogical approaches to environmental education in a model that is "useful in that it highlights the diversity or range of variation in pedagogical propositions in environmental education and thereby contributes to 'celebrating' the richness of this field" (p. 31). Using Sauvé's model, my own research looking at wolf education could fall within the socially critical current field, which focuses on exploring the social issues that underpin "environmental realities and problems" (p. 23). Not only is wolf survival clearly an issue of human attitudes and beliefs, as I have demonstrated above, but I am also interested in the power relationships at play, both in how various human groups weigh in on how wolves are treated, but more importantly, the power relations between humans and the wolves themselves. Sauvé explains how power relations are key for those who approach environmental education from a socially critical stance: "Power relationships in particular are identified and denounced: Who decides what? For whom? Why? How is the relationship to the environment subjected to a dominant set of values? What is the relationship between knowledge and power? Who wields or claims to wield knowledge? To what ends?" (p. 23).

While environmental education has diversified its approaches and the discourses within which it operates, one consideration that remained at the margins until very recently, even in socially critical environmental education, was speciesism (Kahn \& 
Humes, 2009). The "question of the animal" (Oakley et al., 2010) has been barely researched. A growing number of researchers are proposing that questions about humananimal relations and animal advocacy warrant greater attention within environmental education (Fawcett, 2013; Kahn \& Humes, 2009; Oakley et al., 2010). Fawcett (2013), for one, worries that within environmental education, critical engagement with ideas about anthropocentrism, biocentrism, and ecocentrism remains at the level of implicit assumption rather than deemed worthy of serious and sustained "reflexive dialogue" (p. 410). Nonetheless, she shares some recent examples of places where discussion and work around what she calls "naturecultures" are emerging. One example is a recent session during the $10^{\text {th }}$ Seminar in Health and Environmental Education Research in May 2009 dedicated to the question of the animal in environmental education research, which later led to a co-authored paper of which I was part (Oakley et al., 2010). The authors represent a growing number of environmental education researchers who are striving "to position other species as subjective stakeholders in our work and as beings for whom our research matters" (p. 89). Similarly, in 2011, a special issue (volume 16) of the Canadian Journal of Environmental Education was dedicated to articles addressing the topic of "animality and environmental education." I will discuss some of these articles, along with other examples from the emerging field of critical human-animal relations work in environmental education below. First, however, I provide a brief overview of humane education.

\section{Humane Education Overview}

Humane education emerged around 1870 alongside the formation of humane societies created to protect the interests of both children and animals (Kahn \& Humes, 
2009). In the $20^{\text {th }}$ century, as social services for children became more common, the focus of humane societies narrowed their scope, predominantly focusing on domesticated pets and issues of cruelty and/or abuse (Kahn \& Humes, 2009; Selby, 1995). In the 1980s and 1990s, the educational scope broadened with concerns around zoos, aquariums, circuses, and wildlife gaining consideration, led by educators such as David Selby and Zoe Weil who discussed how a multiplicity of issues could be wrapped up with animal rights, including environmental problems and human rights (Kahn \& Humes, 2009).

According to Selby (1995), the dominant mission of humane education today is to teach kindness and compassion towards others, especially animal others. He argues that learning compassion and kindness can have "a range of positive spin-offs in terms of prosocial attitudes towards people of different gender, ethnic group, race, culture or nation" (p. 3). Weil (2004) describes other goals of humane education including: providing students with accurate information about the situation of other animals; helping them develop skills in curiosity, creativity, and critical thinking; engendering feelings of respect, reverence, and responsibility hopefully leading to kindness; and finally, empowering students by presenting them with a variety of possible choices that benefit the earth and all its animal inhabitants.

I draw from humane education in my own research because it offers a conceptual approach that not only considers the situation and treatment of other species as groups, but also the situation for individual animals within species. Since I set out knowing that the wolf educators I planned to interview were likely interacting with individual wolves (at the programs with captive wolves), a conceptual approach that endeavoured to explore 
the circumstances and experiences of individual animals seemed appropriate. Further, I appreciate how humane education probes how various oppressions, both social and environmental, overlap and interconnect — and importantly for my work, includes oppression of animal others in the amalgam (Humes, 2008). Like Kahn and Humes (2009), I worry that "the majority of the socio-ecological turn in environmental education has failed to integrate nonhuman animal advocacy as a serious educational issue" (p. 179). Kahn argues that a "total liberation pedagogy" has potential as it "attempts to work intersectionally across and in opposition to all oppressions (including those of nonhuman animals) and for ecological sustainability" and "views oppression in systemic and complex terms" (p. 181-182, italics in original).

Having explored some of the historical and philosophical foundations of both environmental and humane education, I turn now to research conducted within these fields that is relevant to my own work looking at the experiences of wolf educators.

\section{Environmental Education Research Focused on Wildlife}

In this next section, I delve into research that specifically considers education about wildlife. First, I outline studies that explored the experiences of wildlife educators. Second, I look at research focussed on the value and outcomes of wildlife education for participants; and third, I provide an overview of studies that consider education about wildlife through a sociocritical lens.

The experiences of wildlife educators. While it was difficult to locate research looking at the experiences of wolf educators, I did find a handful of studies that considered the experiences of wildlife educators that have some relevance. For example, Taylor and Caldarelli (2004) explored teaching beliefs of informal environmental 
educators that worked in state and local parks in the northwestern United States.

Similarly, Evans, Ching, and Ballard (2012) looked at the experiences of volunteer trail guides involved in education at a nature reserve in California. Mony and Heimlich (2008) explored conservation messaging about wildlife and the experiences of docents at a zoo in the Midwestern United States, and finally, I discuss Ardoin and Heimlich's (2013) study that explored the views of over 600 educators and practitioners from government agencies and NGOs involved in education and outreach for the purposes of land and wildlife conservation and management.

In both the Taylor and Caldarelli (2004) and Evans et al. (2012) studies, the authors were focusing on the educators' teaching beliefs, perceptions about their roles, and their experiences and practices as educators working in wildlife/environmental education. While Taylor and Caldarelli (2004) found park educators' ideas about teaching and learning were varied, in general the educators in their study were responding to the outdoor, informal context of their teaching environment by focusing on what they felt was a flexible, fun, and more spontaneous pedagogy than occurs in more formal settings like school classrooms. These educators described a practice that included an emphasis on "individual difference, hands-on learning, and the importance of ensuring a sense of comfort and safety for the learner" (p. 463). However, they engaged in some seemingly contradictory practices. For example, on the one hand, the educators preferred an approach that was experiential, hands-on, participant-centered, and adaptive to participant interests yet at the same time there was "emphasis on the nature of knowledge as external, something that is often passed from the park educator to the participant" (p. 
463 ) including certain pre-planned content delivered through a transmission model of pedagogy.

Similarly, the guides that Evans et al. (2012) interviewed and observed described their roles as including expertise/knowledge sharing, "facilitating outdoor experiences," and "promoting stewardship practices" (p. 396). The authors noted that there was sometimes a tension between the roles guides imagined for themselves. For example, some successful guides saw their role as generating enthusiasm and a connection to the reserve and did not feel pressure to be an expert in environmental science. Others, including those who had an academic background in science, felt inadequate in their positions due to their lack of broad knowledge because they envisioned the educator/guide role as that of expert. Evans et al. (2012) noted that not all guides' ideas about possible roles were limiting and some did embrace "multiple perspective[s] on the role of an educator" (p. 399). They concluded by asserting the importance for guides to understand that there are multiple approaches and skill sets applicable to educating/guiding. While my focus of my research was on the learning that emerged from wolf educators' experiences, my participant educators did discuss their roles as wolf educators, so some of the findings in these two studies are applicable.

Moving on, Mony and Heimlich (2008) conducted a two-phase study at a zoo in the midwestern United States using both interviews and observations of zoo visitors and docents, focusing on the types of messages that were both being communicated and received. Interestingly, while all of the docents interviewed in phase one felt their role was to educate "visitors about animals and conservation" (p. 155), when they were observed, the docents were found to be conveying very little of the prescribed 
conservation messages. Instead, they were mainly communicating facts about the animals "relating to age, weight, diet and behaviour of animals in the exhibit" (p. 156). The authors concluded that the docents" "limited awareness of institutional messages hinders their ability to communicate these messages to visitors" (p. 160).

And finally, also related to the experiences of wildlife educators, Ardoin and Heimlich (2013) used surveys, interviews, and discussion groups to uncover practitioners', nonformal educators', and decision- makers' ideas about the utilization of outreach and environmental education in meeting various conservation goals. They found that participants' definitions of "education" varied widely; some had nuanced and multifaceted understandings while others saw it as merely transmitting information. All three groups were interested in learning more about models of effective education that were simple and straightforward to employ and case studies that had shown education as effective part of meeting conservation goals, as well as receive training in both the theory and methods of evaluating education's effectiveness. Providing such professional development can be challenging, of course, since there is no one-size-fits all model or tools.

Wildlife education studies: Participant outcomes. A number of studies have investigated the value of conservation and wildlife education, specifically examining changes in participant behaviour and/or attitudes and/or knowledge. While my study focussed on educators' experiences working with and educating about wolves, this research is relevant in that my participant educators did discuss their beliefs about the role and potential of education for wolf conservation. For this reason, I briefly report here some studies that considered the impact wildlife education might have on 
participants' values/beliefs/knowledge/actions. While I do not go into the details of the studies here, I outline briefly some of the findings from a few studies where wildlife species were actually present.

Orams (1997) used an experimental education treatment to see if changes occurred in tourists' enjoyment, knowledge, environmental attitudes, intentions, and behaviours after viewing and interacting with wild dolphins. He found that those who participated in an education program as part of the tourist experience were more likely to later carry out conservation-minded or "green" behaviours such as removing beach litter, becoming involved in environmental issues, and making donations to environmental organizations as evidenced by both post-experience surveys and a short follow-up interview three months after the experience.

De White and Jacobson (1994) and Visscher, Snider, and Stoep (2009) both explored the influence of educational programs at zoos. De White and Jacobson (1994) asked 1, 015 fourth grade students to fill out questionnaires that measured knowledge and attitudes towards wildlife before and after a visit to a South American zoo. They found that the students whose teachers had participated in an accredited educational course had increased knowledge of and improved attitudes about wildlife conservation. The results led de White and Jacobson to speculate that simple exposure to wildlife at zoos or similar wildlife centres does not necessarily result in learning without supplementary and supporting education.

Visscher et al. (2009) conducted an exploratory study at the Potter Park Zoo in Lansing, Michigan to investigate the impact of interpretation on knowledge retention. Three groups of fifth grade students witnessed an animal training session where one 
group received an interpretive presentation, one received a "facts only" presentation, and the control group merely observed the training session. The group that received the interpretive presentation retained the most knowledge when given a quiz immediately following. They conclude that, "how information is conveyed is just as important as what is being taught, especially with regard to short-term knowledge gain” (p. 494).

These three studies, and others like them that explore the impacts of wildlife education (e.g., Bogner, 1998; Caro, Mulder \& Moore, 2003; Dettmann-Easler \& Pease, 1999; Glick \& Samarapungavan, 2008), suggest that while education can have an effect on attitudes, knowledge, and behaviours when it comes to wildlife and conservation, there are many factors to consider: the length of the education program, the manner in which the knowledge is presented, whether or not the program is residential, as well as the effect of supplementary and supporting education. These studies are interesting for me on a superficial level; for one, they are amongst the small number of studies that actually consider education about wildlife at all. But while they all conclude that education can have an effect on attitudes, knowledge, and behaviours towards wildlife, there are a host of factors influencing attitudes and behaviour that were not considered in either the design or conclusions of their research. As Kollmuss and Agyeman (2002) discuss, all of this research has "some validity in certain circumstances" but "the question of what shapes pro-environmental behaviour is such a complex one that it cannot be visualized through one single framework" (p. 239). Further, Liddicoat and Krasny (2013), having reviewed a substantial amount of research looking at the long-term impact of environmental education, point out that there are many factors that are not considered when studies only evaluate the impacts of programs and experiences shortly afterwards. 
These environmental education experiences may have longer term effects on people, but they are hard to measure especially when you account for "supportive social and political structures, social norms that differ with age cohort, cumulative experiences" (p. 295). Similarly, when looking at free-choice environmental educational experiences, Dierking, Falk, and Storksdieck (2013) discuss the difficulty in assessing the impact of a single experience (e.g., a visit to a zoo) when the visit most likely is just one of a host of experiences that add "to what a person already understands, knows, and feels about a particular topic, behaviour, or attitude, as well as the connection that this effort has to other experiences in the learner's life, both before and after" (p. 362).

Moving now to studies that examine wildlife education in more depth and from a more critical standpoint, the five studies I describe in the next section uncovered nuances that add more to the picture of what might be going on in wildlife education. They employ approaches more akin to my own study of wolf educators, with more emphasis placed on the role and experience and/or situation for the other animals involved.

Wildlife education research: Sociocritical approaches. Russell (2001a) used a mixed methods case study design (consisting of surveys, interviews, and observations) to understand better the educational aspects of whalewatching in Tadoussac, Quebec. In part, she was interested "in the potential of nature experience to disrupt anthropocentrism" (p. 56). Assuming nature is at least partially a social construction, she expected that whalewatchers' interpretations of their experiences would vary and that the whalewatching experience itself might not automatically lead to education or learning; as she wrote, "[c]ontact with whales does not automatically contribute to an anti-whaling position” (p. 66). Russell (2001b; see also Russell \& Hodson, 2002) found that 
whalewatchers at her site wanted more education about not only whales, but also their larger ecological community. Further, they desired more information about the impact of human communities and whalewatching itself on the whales. She describes how, for the most part, the whalewatchers did not need convincing about the need to conserve whales: "These whalewatchers were ahead of us in that regard, and were thus disappointed by the lack of emphasis on whalewatching as a catalyst for activism" (pp. 249-250).

Warkentin (2011) also studied marine ecotourism, contrasting and comparing three sites that advertise human-dolphin interaction in the form of swim-with-dolphin experiences. She describes how, depending on the program, different levels of interspecies protocol were stressed. Two of the sites "effectively afforded engagements in a kind of interspecies etiquette" (p. 117). At both these sites (one captive and one open-ocean), the tourists were under strict direction not to touch the dolphins and neither of these sites used food to entice or control the dolphins, leaving them some choice in whether and how to engage with the human participants. Warkentin contrasts this with a third site where the experience felt mechanical and highly scripted as trainers with food rewards controlled the dolphins' actions; the human participants' actions were also strictly coordinated, leaving little room for any kind of authentic or spontaneous interspecies interaction. At this third site, professionally taken photos that tourists could purchase following the experience seemed to be a central focus; the human's experience was given priority over the other animals, making the experience feel highly commodified. Warkentin encourages readers to think about the ethics related to interspecies experiences such as these, especially "the kinds of meeting places we might imagine and create with ethical affordances as our guide" (p. 117). 
Watson (2006) also looked at human-wildlife interactions, in this case by investigating the experiences of 14 children at an Ontario summer camp. Using a phenomenographical and ethnographical method, he observed and discussed nature experiences with summer camp participants. He found that common wild animals such as frogs, toads, snakes, salamanders, insects, and fish were agents through which the children came to realize their connection to place. Watson believes these experiences with common wild animals also acted as a "conceptual handhold" (p.135) by which children came to understand more abstract concepts about the "other" such as the agency of other animal beings. This research led Watson to propose that embodied experiences with common wild animals, often encountered during unstructured free time, can be an important stepping stone for children in developing understanding of the larger natural world. He concluded that experiences such as these can be fostered in urban and suburban, not just summer camp environments.

Like Watson, Fawcett (2002) also explored children's perceptions of common animals, in this case, bats, frogs, and raccoons. She had children in kindergarten and fifth grade draw pictures and tell stories about these animals—-half of the children had a twenty-minute direct encounter, the other half did not. She found that children, particularly kindergartners, who had direct experience with the animals were less likely to tell stories that contained fear, misconceptions, and anxiety, and were more likely to tell stories about friendship and kinship. With the group of younger children especially, the animals were storied as having agency and subjectivity that was both similar and different to themselves. In part, the research prompted Fawcett to conclude the "importance of 
direct experience for positive human and other animal relationships, and ... for biological conservation and environmental education" (p. 131).

Like Fawcett, Pendersen (2010) conducted her research within a formal education setting. Taking a critical stance, she investigated human-animal relations within the context of secondary schools in Sweden, conducting observations, investigating curriculum, and talking with students and teachers. She especially spent a lot of field time observing in vocational animal caretakers programs that housed various animals on site as well as had students take field trips to facilities such as zoos and aquariums. Her fieldwork led her to stress that "practices and rationales of human-animal relations" (p. 122) within education cannot be considered outside their social, political, and historical contexts although in practice, actually doing so was the exception rather than the rule. She found that very often, the school processes reinforced certain anthropocentric ideas about other animals and human-animal relations. For her, attending to schooling's hidden curriculum about human-animal relations is necessary and she argued should be explicitly addressed.

Some of the studies described above have significant relevance to the experiences of the wolf educators in my study. For example, as I will discuss in the findings and discussion chapters, similar to Warkentin (2011) and Russell's (2001a) participants, the educators I interviewed had contemplated the ethics of their interspecies encounters. As well, as in Watson's (2006) and Fawcett's (2002) studies, the subjectivity and agency of other animals (in this case, wolves) emerged as an important consideration. Finally, like Pendersen (2010), I found that most of my findings about wolf educators and wolf education could not be understood outside their social, political, and historical contexts. 


\section{Humane Education Research}

In the section that follows, I describe three studies that I found to be relevant to my own, one of which is my MEd thesis research. Each took a unique approach to conducting research from a humane education perspective.

Pedersen (2011) examined the experience of an undergraduate science student conducting a cognitive bias experiment with 33 battery hens. Pedersen described the subjectivities and human-hen relations that emerged between the student and the hens that could not be captured within the quantitative experiment the student was conducting, leading her to ask, "What happens to education and learning when the human subject is decentered and nonhuman animals are allowed to emerge as subjects, rather than as objects, tropes, or species representatives?” (p. 17). Whether or not our research designs allow for the researched animal's subjectivity to emerge, their subjectivity exists nonetheless, which is a good reminder that research design and representational choices have the power to work for, or against, the situation for animal others.

MacPherson (2011) examined the potential for humane education from a very different angle, but like Pedersen (2011) also considered the issue of animal subjectivity. Using her relationship with her companion dog Tashi as an example, she explored possibilities for humane education through human-companion animal relationships. She concluded that these relationships involve significant learning that might be translated to the larger context of animal and environmental exploitation. She explains that while some research shows many of us know about environmental problems, yet still remain inactive, engaging in the emotional worlds that can exist between humans and companion animals might provide learners "a deeper understanding of their personal, felt 
identifications and relations with the greater-than-human world" (p. 89) that could better lead to action. MacPherson argues that rather than focusing on science and factual information alone, emotional-social learning experiences can help diversify our approach and perhaps offer a more effective means to engaging with topics related to our relationships with non-human animals and the environment generally.

MacPherson’s (2011) work resonates with my own previous MEd research on musher-sled-dog relations (Kuhl, 2008; 2011a). For one, the mushers I interviewed in my study discussed how the skills they built working with dogs could be transferred to an improved ability to work with other people; mushers explained that "they had developed empathy, had learned compassion, were able to endure chaos and stay calm, and had learned patience" (2008, p. 78). Mushers also felt that working relationships with dogs had given them a window into the amazing capabilities of another species. One could conclude that, in part, mushers are participating in a form of humane education, and in doing so, are learning compassion, appreciation, respect, and empathy.

In the findings and discussion chapters that follow, I will return to some of these studies and ideas discussed here as they connect to some of my participants' ideas and thoughts about wolf education.

\section{Education and Wolves}

There is a paucity of research looking specifically at wolf education, including the experiences of wolf educators. This is unfortunate when you consider the fact that wolf education has been in place as a recovery strategy for quite some time (Frits et al. 2003; Troxell et al., 2009). As noted in the literature reviewed in the sections above, wolf- 
human relations are complex and involve historical, environmental, political, sociocultural, attitudinal, and ethical dimensions. It is not a leap, then, to suppose that education about wolves will itself include a tangle of these dimensions. Questions such as who is offering the education and for what purpose, what messages are being communicated (both explicit and implicit), who is funding the education and for what reasons, who are the participants and what beliefs and values they come to the program with, and what educational methods are being employed will all undoubtedly affect the process and outcomes of wolf education. The limited research within the subject area has hardly considered any of these questions, leaving possibilities for wolf education research wide open. In the following section, I will attend to the little research I was able to unearth that pertains to wolf education. I first describe literature specific to wolf education, followed by a review of some research where education was described, discussed, or included as a small part of a larger study.

\section{Literature Specific to Wolf Education}

In this section I discuss two academic book chapters and three studies specific to wolf education. First, in a book focussed on wolf recovery and human attitudes towards wolves, Taylor's (2009) chapter examines wolf education as a tool for conservation through a lens of transformation theory. She believes transformation theory is a good fit for wolf education given that there are so many complex aspects in the debate about wolf survival, including social, political, and cultural. She explains that the goal of wolf education if considered in light of transformation theory is to "foster awareness and reflexivity" (p. 168) with the aim of transforming negative beliefs and actions towards wolves while still carefully maintaining respect for the learner's autonomy. Changing 
attitudes then becomes a process that must consider the various values and beliefs of the learners. She stresses that those who have negative attitudes towards wolves are not necessarily a homogeneous group, "but rather a complex mix of different social groups with different socio-political and cultural backgrounds" (p. 162). Consequently, those who are in the role of wolf educator need to be aware of "the highly complex factors and situations that exist in the conservation of a species that continues to provoke such polarized perceptions and attitudes" (p. 164).

When it comes to the role of wolf educators, Taylor (2009) believes it is important that educators explore and examine their own beliefs when it comes to wolves before they set out to educate those who might have a very different set of values. She also stresses "that conservation educators understand distinctions between education, advocacy, indoctrination, and propaganda" (p. 165). Learners should be encouraged to explore their own beliefs and think critically rather than being fed propaganda. She writes:

Conservation educators do need to be aware of the potential dangers for indoctrinating their own values and beliefs into others, especially children and be mindful about the true purpose of education, which is to encourage critical thinking and reflexivity in order that the pupils of learners can arrive at their own value judgments. (p. 165)

In part, this means that educators should remain open to the fact that, even after attending an educational program, learners may continue to have opinions and ideas that are contradictory to their own.

In another book about wolf recovery, this time focused on the Great Lakes region of the United States, one chapter is authored by individuals involved in education and outreach efforts (Troxell et al., 2009). They provide an overview of six educational wolf 
programs in the region, but these are generally descriptive and do not offer much analysis of the programs. However, Troxell et al. do make some interesting statements and conclusions based on their cumulative knowledge and experience with wolf education. Contrasting an early trend when educational messages were fairly black-and-white (i.e., wolves are cool) with contemporary wolf education in which education "was broadened to include moral and ethical considerations concerning wolves" (p. 299) as well as information rather than answers, they state: "Education about wolves needs to help people to wrestle with difficult and controversial questions - but should not try to provide them with answers" (p. 298). Troxell et al. (2009) go on to argue that given wolf populations in the region have grown, education now should focus on helping people learn how to co-exist with wolves: "As we look to the future, education will need to be less of a lesson plan on wolf ecology and more about how we value the wolf and the environment. Education will be necessary for coexistence between wolves and humans to occur” (p. 308). Troxell et al.'s beliefs about wolf education, both content and delivery, relate directly to some of the ideas I explored through this research.

Turning now to studies that have specifically investigated wolf education, first, Black and Rutberg (2007) used a survey and a pre- and post-test design to examine how effective wolf education was in changing attitudes and knowledge about wolves. They used as their case a wolf centre in Indiana called Wolf Park. They surveyed a total of 377 adults and determined that, overall, education that included exposure to wolves improved attitudes and knowledge about wolves. The authors found that changes in attitudes towards wolves and changes in knowledge were independent of each other; in other words, an improvement in pro-wolf attitudes did not necessarily relate to an increase in 
knowledge, or vice versa. They also ascertained that the regions where the visitors resided made a difference. Those who lived closer to wolf populations and those who visited having already formed predetermined negative opinions about wolves were less likely to show much positive attitude increase. While, in general, participant attitudes improved after the visit, the authors acknowledged that most visitors to the education centre arrived with positive attitudes. This led Black and Rutberg to suggest that in order to reach members of the public with more negative attitudes, other strategies such as centres or programs that include a broader range of animal species might draw a more attitudinally diverse population of visitors.

Like Black and Rutberg (2007), Willard's (2008) master's study looked at wolf education specifically. She interviewed 20 individuals who were involved in education and outreach efforts related to the wolf management debate in the western United States, asking the question, "What is the role of education in reducing conflict in light of the issues emerging from the value-based nature of the wolf management debate?" (p. 23). Her study included a wide range of participants with interviewees coming from organizations dedicated to conservation advocacy as well as those advocating for the wolf to be delisted as an endangered species, along with educational organizations, wildlife management agencies (state, federal, tribal), and media groups. Participants reported a variety of experiences, approaches, and goals with regards to education and public outreach. For example, educators from conservation groups seemed most interested in achieving wolf restoration while participants from educational organizations more often aimed to build appreciation for wolves. The participants highlighted the challenges of educating about wolves and wolf management issues given their value-laden and 
complex nature as well as difficulties in assessing the effectiveness of their efforts. Willard writes, "Every subject referred to the difficulties of providing education and outreach about an issue that stirs up such intense emotions grounded in a multitude of unyielding opinions" (p. 38).

Willard (2008) made three general conclusions. First, that education has a capacity to "portray the complexities of the debate by presenting scientific information alongside social definitions" (p. 33). Many of her participants discussed the importance of offering participants biological information along with a diversity of viewpoints about wolves and wolf management. Second, Willard concluded that education could play a role in transcending some of the communication barriers surrounding the wolf debate. She writes that strategies such as, "[h]umor, stories, and unique experiences have been shown to capture the audience's attention, break through communication barriers and create long-lasting impacts to individuals' perceptions of environmental issues" (p. 58). Third, Willard suggests that education and outreach are a means to offer people opportunities for new experiences, "opening the door to greater understanding of the conflict's complexities" (p. 33).

The third wolf education study I found was by Samuelson (2012) who conducted quasi-experimental research to assess visitor attitudes towards wolves at one conservation centre in Montana (The Grizzly and Wolf Discovery Center) at the edge of Yellowstone National Park. Using surveys, she assessed adult visitor attitudes before entering, after their visit, and one month after their visit. She also did a content analysis of the centre's information regarding wolves. She concluded that, on the whole, the centre offered a balanced viewpoint of wolves. They were depicted as both solitary and social. They 
were also represented as having both negative and positive interactions with humans, one display showed people protesting wolves' reintroduction to Yellowstone. When it came to visitor attitudes, she concluded that they "did [positively] increase slightly during the visit and time spent viewing the wolves in their enclosures was significantly correlated with this attitude change" (p. 83). While this attitudinal shift lasted the month, its strength decreased some over time. Interestingly, there was no significant correlation between attitude change and the materials participants read at the centres (for example, interpretive signage about wolves). Also intriguing, the visitors self-reported a greater change in attitudes than was actually measured by the survey's questions. As well, Samuelson (2012) found that with an increase in knowledge came a decrease in confidence about attitudes. She stated: "These results indicate that the more an individual knows about wolves, the less confident they are about their attitudes towards them" (p. $53)$.

\section{Research and Literature in Which Wolf Education is a Component}

Black and Rutberg's (2007), Willard's (2008), and Samuelson's (2012) were the only studies I found that focused specifically on wolf education. Nonetheless, there are descriptions and discussions about education in other studies, particularly in their conclusions.

Fritts et al. (2003) describe how using education to influence attitudes in the hopes of aiding wolf recovery in the United States began in the 1970's. All of the wolf recovery plans written at that time recommended public education as a means to encourage wolf conservation. When initiating wolf reintroduction in Yellowstone National Park, for instance, wolf biologists spent around $60 \%$ of their time on "some 
form of public outreach" (p. 297). Fritts et al. argue that it is important to give the public realistic and objective facts about wolves and wolf-human conflict as well as possible alternatives and solutions. While I personally find their reliance on "facts" problematic given all information is embedded in a historical and/or sociocultural context, I nonetheless agree with their assertion that counterpoints to sensationalized accounts in the media are needed.

Switalski et al. (2002), in an article about public involvement in wolf conservation in Utah, also believe education to be an important management and conservation strategy. Like Troxell et al. (2009), they do not think public education messages should be onesided, rather they argue that many aspects, issues, and points of view should be incorporated. They also recommend that various stakeholder agencies be involved and represented and anticipate that if wolf populations grow in Utah, the goal of wolf education should be to help people "become more knowledgeable and objective about wolves and wolf management” (p. 37).

Andersone and Ozolins (2004) also briefly discuss the topic of education in their survey that looked at the attitudes towards three carnivores in Latvia. Fascinatingly, they suspect that wolves and bears having endangered status might lead to more public awareness and knowledge about those animals. They write that "the status of an endangered animal might favor the knowledge of the species, raising interest and probably even improving the attitudes towards it, whereas a species considered a common pest, like the wolf, can suffer from intense persecution and have a negative image" (p. 186). In surveys of 401 people, they found that sources of information on carnivores included documentary films, magazines, newspapers, biology lessons, and 
fairy tales. The majority of respondents surveyed reported that they would be interested in receiving more information about large carnivores in magazines, newspapers, television, and radio.

More loosely related to the subject of education, Ericsson and Heberlein (2003) also considered the relationship between knowledge about wolves and attitudes in their survey of hunters and the general public in Sweden. They found that, generally, when individuals had more knowledge about wolves, attitudes tended to be more positive. This is a similar finding to that of Naughton-Treves et al.'s (2003) study of Wisconsin rural residents where higher levels of education did correlate with improved tolerance of wolves. Ericsson and Heberlein (2003) found that, overall, people do not have strong attitudes towards wolves and do not seek out information on the topic. Among those few who do have strong opinions, while they may seek out information, they are unlikely to change their strong beliefs. They concluded that while there is potential for education to improve attitudes, like Black and Rutberg (2007) suggest, an effective method for reaching target populations is needed. Smith (2006), who studied the attitudes of paddlers in Pacific Rim National Park Reserve, Canada, towards wolves would agree. She suggests that simplistic educational efforts may not have much effect as attitudes stem from a complex interplay of beliefs and emotions. Instead, she argues that education needs to acknowledge that "a variety of dynamic, often deeply rooted, social structures and norms" (p. 131) are involved.

To conclude this section, education is often recommended as one wolf conservation strategy despite the fact that its effectiveness (or for that matter, any other aspects of wolf education) has barely been researched. As Andersone and Ozolins (2004) 
and Willard (2008) observe, "education" about wolves can come in many forms such as documentaries, newspapers, websites, magazines, children's stories, presentations, experiential learning, school classrooms, and visits to wolf education centres. We can also conclude from other literature reviewed in this chapter that human attitudes about wolves are complex and by no means simple to unravel. Culture, socioeconomic status, class, gender, level of education, occupation, age, location, and region each influence people's attitudes when it comes to wolves. Perhaps this is why some wolf educators emphasize the need to include social issues and perspectives alongside wolf biology in their programs (Troxell et al., 2009; Willard, 2008).

The understanding gleaned from this literature review has proven useful to my research in a variety of ways. For one, the literature on human-wolf relations and wildlife education provide context and a better understanding of the climate in which wolf programs emerged and within which educators are working. As well, my participants' experiences educating and interacting with wolves, and the meaning and learning they drew from these experiences, was undoubtedly impacted by the many factors discussed above. 


\section{CHAPTER THREE}

\section{METHODS}

In this chapter, I describe the methodology, lens, and data collection methods I employed as I investigated wolf education from the perspective of educators, particularly their experiences both teaching about, and working with, wolves. While this study primarily engaged a qualitative phenomenological interview methodology, I also explain below why and how I drew from critical theory. I detail my methods, including my participant selection and interview process, along with how and why I supplemented the interviews through conducting observations, creating program/centre overviews, and including participant photographs. Finally, I discuss my methods of analysis as well as the limitations and ethical aspects of the study.

\section{Methodology}

\section{Qualitative Study Design}

A qualitative, phenomenological interview-based study making use of a critical lens was the approach I took to penetrate the layers and facets of wolf education from the perspective of 17 wolf educators, including what they had learned about wolves through working with them. Creswell (2012) suggests that qualitative research is best suited for research where one wishes to "obtain a deep understanding" (p. 19) or to develop “a detailed understanding of a central phenomenon" (p. 16). As discussed in the literature review earlier, how people feel about wolves and their conservation is not simple; rather, it is a complicated, messy issue. Educators who work at wolf programs possess deeply rooted ideas and beliefs about wolves that no doubt colour what they subsequently teach 
and learn. A qualitative design, then, seemed most appropriate for this investigation where I hoped to unearth some of the social and cultural aspects of educators' experiences with wolves and wolf education.

\section{Phenomenology}

At its core, phenomenology entails studying the lives of those who have experienced similar phenomena (Lichtman, 2010). According to Van Manen (2002), the shared phenomenon in question could take many forms and he gives a variety of examples in his book, including phenomena that are common, uncommon, ritualized, aesthetic, technological, and ambiguous (to name a few). Emerging in Europe around the time of World War I, phenomenology has roots in both philosophy and existentialism. While Kant may have been the first to use the Greek term "phenomenology," most people accept Husserl as the "father" of phenomenology (Lichtman, 2010). Phenomenology can be seen as both as a philosophy or a method, and today is approached in a multitude of ways from traditional to broad and interpretive; however, at the core, phenomenology is always seeking to understand some aspect of lived experience (Lichtman, 2010).

There are two main reasons why I chose to pursue a phenomenological interview study. First, I believed phenomenology was the best methodological fit for my research question: What are the experiences of educators who work at programs that feature captive or wild wolves, in particular, what do they learn through both working with and teaching about wolves? Second, phenomenology has a history and precedent amongst human-animal relations researchers as a methodology that allows for acknowledging the sentience of non-human animals, thus offering a framework from which to consider relations between two species (which I discuss more thoroughly below). 
In the case of my study, I was interested in the lived experiences of wolf educators: both their experiences working with wolves and their experiences educating. But as both Seidman (2013) and Van Manen (2002; 2014) explain with regards to phenomenological methods, it is not only the experiences themselves that the phenomenologist is concerned with but also the meaning participants make of those experiences. Seidman writes, "At the root of in-depth interviewing is an interest in understanding the lived experience of other people and the meaning they make of that experience” (p. 9). Similarly, according to Van Manen (2002), good phenomenological studies "provide concrete portrayals of lived experiences" (p. 49) and they also "offer insightful reflections on the meanings of those experiences" (p. 49), hence the second part of my research question regarding wolf educators, what do they learn through both working with and teaching about wolves? At its core, this portion of my question was about exploring the meaning educators ascribed to their experiences.

While investigating various researchers' choices for studying human-animal relations, I found that phenomenology emerged as one of the more common ones (for examples, see Abram, 1996; Russell, 2001b; Shapiro, 1997; Warkentin, 2007; Watson, 2006). Abram (1996), for example, is an oft-cited scholar in human-animal studies. He discusses and uses phenomenology as a methodology that offers avenues to reconnect humans with nature, especially in modern western society. His book, Spell of the Sensuous (1996) is a phenomenological examination of how written language has contributed to modern western society's disconnect from the animated, living world. He explores the ideas of Husserl and how they opened up possibilities for understanding human-nature relationships as intersubjective rather than hierarchical. According to 
Abram, Husserl understood that a focus on objectivity within the sciences "had led to an almost total eclipse of the life-world in the modern era...this living dimension in which all of our endeavors are rooted" (p. 41). Husserl believed science was not purely abstract, but rather grounded in our everyday, sensual, lived experiences of the world. Phenomenology allowed for "a collective landscape, constituted by other experiencing subjects as well as by oneself” (p. 37). Abram suggests that from its early days, then, phenomenology has sanctioned investigating and understanding the world as one inhabited by many subjects, both human and other, thus offering up avenues for studying shared interspecies experiences.

Abram's stance (1996), that non-human animals are also "experiencing subjects," is in contrast to the historical social science perspective where non-human animals were typically objectified, “othered” (see: Emel, 1995; Kuhl, 2011b; Noske, 1997; Russell, 2005), and considered beyond the social realm, in part because they don't use human language (e.g., Mead, 1962). However, more recently, this approach towards non-human animals has been challenged and the study of human-animal relations within the social sciences has increased and gained standing (Fawcett, 2013; Gerbasi, Anderson, Gerbasi \& Coultis, 2002; Shapiro, 2002). Nonetheless, social science researchers, including educational researchers such as myself who wish to give other animals "voice" in their work, are faced with finding frameworks that acknowledge the sentience and agency of other beings while recognizing that some of these very frameworks historically objectified and othered non-human animals in the first place.

While I am cognizant of the fact that other animals may not be aware of, or interested in, research on human-animal relations, they are nonetheless very often 
impacted by the outcomes of it, making our research and representation choices especially important. Russell (2005) states this well, writing that "other beings are likely not remotely interested in our research and writing, busily getting on with their own existence, yet they are profoundly, materially, impacted by our inscriptions" (p. 435). While phenomenology may not be the only methodology that offers avenues to investigate human-animal relations while remaining conscious of these ethical and theoretical concerns, because phenomenology focuses on the lived experiences of individuals (human or non-human), it offered me a methodological framework to answer my research question where both the wolves and humans were prefaced as subjects with agency.

An attitude of wonder. Epoché (or bracketing) and reduction are fundamental to phenomenology (Van Manen, 2014), but some phenomenological researchers have moved away from bracketing at least in how it was originally conceptualized (Lichtman, 2010; Vagle, 2014). While I had concerns prior to my study about the notion that one can truly set aside or bracket one's past ideas about and experiences with the phenomenon in question, I did try to employ a phenomenological attitude that involves trying to put aside my thinking and reflecting about the phenomena long enough to be "swept up in a spell of wonder" (Van Manen, 2014, p. 26). I tried to embrace phenomenology as a method or attitude, then, by opening myself up to curiosity and wonder, and by asking questions as much as seeking to answer them (Van Manen, 2014). 


\section{Lens}

\section{Critical Research}

The lens I incorporated was that of a "critical" researcher. Bogdan and Bicklen (1998) define critical research "as an ethical and political act. Critical theorists agree that their research should empower the powerless and work toward the elimination of inequality and injustice” (p. 271). Many environmental education and human-animal relations researchers point to the similar mechanisms that lie at the root of oppression whether it be racism, classism, and sexism or the oppression of nature and other animals (Bell \& Russell, 2000; Fawcett, 2000, 2013; Gruen, 1993; Humes, 2008; Kahn, 2009). Similarly, in my own work, I attempt to embrace the concept that working to disrupt and eliminate environmental or animal oppression can also benefit humans, including those marginalized through similar mechanisms. For example, one mechanism of oppression is exploitation. When a region is exploited for resources (for example, deforested in order to grow cash crops), very often the negative impacts are not limited to the land, flora, and fauna, but also the local people who rely on the land. Projects that work to conserve a species such as the wolf can also serve local people, benefiting more than one marginalized group/species (see Bizerril, Soares, \& Santos, 2011; Ohlson, Cushing, Trulio \& Leventhal, 2008). Through exploring wolf educators' experiences and insights, I had hoped to understand, at least in part, how humans might learn to coexist with wolves on the land, and how this might ultimately benefit both wolves and humans.

Exploring the idea of critical environmental education, Kahn (2009) writes about the "ways in which power circulates throughout nature and culture, to the systematic advantage of some and disadvantage of others" (p. 182). He believes there are a variety 
of pedagogical opportunities to address these systems of oppression. In the main, he is interested in an approach to environmental education that includes an acknowledgement of speciesism, which I too attempted to address through this research. For instance, considering the research I have reviewed within the field of human-wolf relations, the attempts I made during this study to include and contemplate the experiences and situation of the wolves themselves, at some level, may be unique. For example, my choice to adopt the premise that educators may learn things not only about, but also from, the wolves themselves is an approach I have not come across in any other human-wolf relations research. Likewise, as I lay out some of my findings in the chapters that follow, I discuss the ethical aspects and implications of how we humans think about, teach about, and ultimately treat wolves. Doing so also adds another critical layer to this study.

However, as I lay out the methods I employed in this chapter, I am aware of Russell's (2003) illustration of how sometimes there is a gap between what we wish for methodologically and what our research actually does. How did it make a difference that my lens was critical? I believe that evidence of the critical aspects of my research may be subtle. For example, as a researcher with a critical lens, I tried to remain aware of my own context and how that coloured my interpretations of what I was seeing and hearing. I also tried to remain cognizant of the various sociocultural assumptions that might underlie educators' ideas about wolves and wolf education, both as I interviewed and observed them, and also as I analysed and interpreted the interviews. For instance, I explored with the educators how various cultural portrayals of wolves to which they were exposed growing up (fairy tales, movies, etc.), may have influenced their current ideas and beliefs. Similarly, I also considered how, for some educators, teaching and thinking 
about wolves through a framework of western science may have impacted their interpretations of their experiences with, and ideas about, wolves. And ultimately, from a critical advocacy standpoint, I was, and am, interested in what my research might mean for the wolves themselves. I care deeply about wolves and am committed to helping create conditions for them not merely to survive, but to flourish, and my research was obviously flavoured by this position. Perhaps sharing this research about the insights of educators will offer new perspectives that influence how wolves are ultimately treated.

\section{Participant Selection}

Van Manen (2014) discusses how when applying a phenomenological method, sampling is done in order to gain enough participants that the researcher has adequate rich descriptions, anecdotes, and stories to "help make contact with life as it is lived" (p. 353). Some phenomenologists borrow sampling methods that are used in other qualitative methodologies, but stress the importance that participants be selected based on their potential to provide insight into the phenomenon in question rather than on some empirical formula (Groenewald, 2004; Van Manen, 2014). In my case, participant recruitment took place using a convenience sampling technique (Berg, 2007), a type of purposeful sampling that, according to Seidman (2013), is commonly used to choose participants in phenomenological interview research.

First, using a search engine, I made a list of wolf centres and programs in North America where live wolves were featured. Early on, I located a list entitled "Captive Wolf Facilities in North America" (White, 2014) that was quite helpful although it was not exhaustive nor did all sites listed meet my criteria. Before adding a centre to my list, 
I checked each centre's website to find a description of what they did, making sure it included an intention to educate people about wolves. I initially emailed 16 facilities, sending them a brief overview about my study in the email text, and attaching a participant letter (see Appendix B) with more information. This led to only two responses from potential participants.

Next, I attempted to contact all 16 of these programs and centres by telephone, asking if there was an educator on site who might volunteer, and in this manner was able to recruit more participants. As well, when I started interviewing in June 2014, I asked each educator at the end of the interview for ideas about places that offer wolf education involving captive or wild wolves or other educators they might know who would be willing to participate. I was able to locate a few more centres and participants with this snowball sampling technique (Berg, 2007; Creswell, 2012). I also continued to use a search engine with various terms such as "wolf education," "wolf centre," "wolf center," and "wolf sanctuary" until I was unable to find any more websites for centres/programs online. I did unearth some additional centres using this technique. I started to recruit participants at the end of May 2014, and continued to recruit throughout the interview process up until October 2014. In total, I conducted 16 interviews with 17 participants from 15 different centres/programs. (In the case of two centres/programs, two participants each were interviewed, once as an individual interview and once with two participants in a single interview at their request.)

While I had hoped to recruit participants who had at least two years of experience with wolf education, it was so difficult initially to find interviewees that I accepted any wolf educator who was willing to participate. In the end I was able to talk with wolf 
educators who had anywhere from one to 28 years experience. (Further description of the participants follows in chapter four.) While the goal of phenomenological interview research is not to gain a sample that is representative (Van Manen, 2014), I thought that since cultural and regional context can impact lived experiences it was important to talk to people who came from a variety of regions and a diversity of programs. As demonstrated in the literature review, cultural and regional contexts influence humanwolf relations. Therefore, interviewing participants from different regions (i.e., Rocky Mountain States, western Canada, the Great Lakes region in both the United States and Canada, the southwestern United States) allowed me to highlight some of the unique experiences that were influenced by the region in which the education centre or program was situated. In the end, I was able to recruit wolf educators from thirteen different states or provinces (see chapter four).

\section{How Many Participants?}

The idea of saturation may not be applicable or appropriate for phenomenological studies according to Van Manen (2014) because the goal is not to find commonalities within a social group but rather to explore examples of experiences as they are livedand some of these may be singular and unique. In contrast, Seidman (2013) does discuss the concept of saturation as it relates to phenomenological interviewing. Certainly, I did find myself wondering as I was recruiting and interviewing when an appropriate time would be to stop. Since I was unable to do as much observation as I would have liked, after consulting my supervisor and some resources on interview research (Mason, 2010; Seidman, 2013), I decided to interview a few more participants than the 13 to $15 \mathrm{I}$ had originally intended. Van Manen (2014) suggests that the researcher collect as many 
interviews as needed in order to describe the phenomena under study with adequately rich descriptive anecdotes and stories, stories and anecdotes that give a sense of "life as lived." On the same topic, Seidman (2013) encourages the researcher to consider whether there are enough interviews "to reflect the range of participants and sites that make up the population" (p. 58). He also suggests that if the researcher is unsure, it may be advisable to err on the side of too many interviews rather than too few, because once analysis and interpretation begin, it may be difficult to make meaning from data that "are just too thin" (p. 58).

As I finished interviewing 17 participants, I realised I was very often hearing similar ideas and concepts from my participants and I had the sense that I had conducted an adequate number of interviews. (That was timely since I also was unable to locate any more centres that I had not already contacted unless I started recruiting from centres whose focus was wolf rescue rather than education thereby taking me away from my original criteria.) I also felt reassured by Mason’s (2010) paper discussing saturation in interview research. He reviewed $560 \mathrm{PhD}$ dissertations that employed interview methods using a number of qualitative approaches. Of the 25 phenomenological-based interview dissertations that fit his criteria, he found that the range of interviews conducted was between seven and 89 , with a mode of 20 which was fewer than the modes found in other qualitative approaches. While I felt reassured knowing the number of participants I recruited was close to this mode, I did note that Mason (2010) worried that PhD students such as myself were not fully incorporating the concept of saturation given so many studies reported participants numbering in multiples of 10 (e.g., 20 or 30 participants). He speculated that the $\mathrm{PhD}$ students in question either did not fully understand saturation, 
were choosing to err on the side of larger-than-needed samples in order to more easily defend their work, or perhaps found it simpler or safer to stick to whatever their proposed number of participants happened to be rather than seriously engage with the concept of saturation. Whatever the case, even though the concept of saturation may not be an appropriate consideration for phenomenological interviewing (Van Manen, 2014), I nonetheless feel that I talked with enough wolf educators to collect a sufficient base of ideas, stories, and anecdotes to uncover some of the facets and nuances related to the experience of teaching about, and working with, wolves.

\section{Data Collection Methods}

This next section will provide an overview of data collection methods. Phenomenologists use various data collection tools developed in the social sciences but take on a phenomenological attitude with a goal of exploring "examples and varieties of lived experiences" (Van Manen, 2014, p. 313). A few empirical methods mentioned by Van Manen include "interviewing for experiential accounts, observing experiences, identifying fictional experiences, and exploring imaginal experiences from other aesthetic sources" (p. 312), and I indeed have used several of these.

Data collection for this study primarily involved in-depth interviews, a common tool used by phenomenologists (Groenewald, 2004; Van Manen, 2014). However, given the importance of context when it comes to understanding human experience as lived (Vagle, 2014; Van Manen, 2014), the interviews were supplemented by conducting a brief content analysis of each wolf centre's website. I also conducted observations at two sites since Van Manen (2014) suggests the best way “to enter a person's lifeworld is to 
participate in it" (p. 318) and close observation can help a researcher explore the phenomena in question (in this case, the experiences of wolf educators). Finally, I included an aesthetic component (Vagle, 2014; Van Manen 2014) in data collection when I encouraged all of the participants to share photographs.

\section{Interviews}

Johnson and Rowlands (2012), in their discussion of in-depth interviewing, explain that it is useful when one wants to delve into the layers of a complex question "to penetrate to more reflective understandings about the nature of that experience" (p. 102), which aligned well with my intention to seek deeper understandings of wolf education and educator-wolf relations. I conducted 16 interviews ranging from 50 minutes to two hours, with 17 educators (one interview involved two educators simultaneously at their request) who worked or volunteered for a variety of wolf education programs around North America. Ten semi-structured interviews were conducted over the phone and six through Skype. I used a loosely devised interview guide (see Appendix A).

I relied fairly heavily on Seidman's (2013) book concerning phenomenological in-depth interviewing. Seidman writes, "Phenomenology is a complex philosophy with many facets. There is no single approach to interviewing research that could be called phenomenological" (p. 15). Despite this, he does offer a guide for those wishing to conduct interview research from a phenomenological stance. He urges the researcher to consider the following: First, lived experience is both temporal and transitory in nature. The goal, then, is to come as close as we can to understanding our participants' experience at a certain place and point in time. Second, phenomenological approaches focus on the subjective point of view of participants. Since we cannot be another person, 
we can only do our best to understand their subjective point of view about the experience or phenomena in question. Third, in a phenomenological interview study, there is a focus on the meaning making aspects of experience. We are trying to get at the essence of a certain experience through reflection after the "lived experience" has actually happened. And, finally, Seidman (2013) discusses the importance of context. He writes, "context is crucial to understanding the meaning of participants' experience from their point of view" (p. 19). Ideally, the interview itself helps to put the actions and behaviours of participants' lived experience into context, without which we may not be able to understand the meaning for them of the phenomena in question, in this case the experience of educating about, and working with, wolves.

How did I incorporate these assumptions into the interview process in my investigation? For me, these had an impact on all aspects of the interview study and, as Seidman (2013) suggests, affected "the structure, technique, and approach to analyzing, interpreting, and sharing interview material" (p. 19). For example, the goal of the interviews was to capture educators' perspectives about their experiences with wolves and wolf education, and the meaning they attach to these experiences, ensuring that these experiences were given context. I found giving the interviews context to be the most difficult task, especially as it related to place, because six of the educators wished to remain anonymous. Still, all but one participant felt comfortable with me discussing the possible interplay between where their centres were located (at least the general region) and their experiences with wolf education, allowing me to make connections between place and lived experience. I also attempted in the chapters that follow to focus on, and discuss their experiences as meaningful in and of themselves rather than discussing only 
those experiences that were common amongst the group (Seidman, 2013; Van Manen, 2014). During the interviews, I often asked the participants to share an anecdote or a story when they made a point or answered a question in an attempt to bring the discussion back to the particular, making an effort to draw out recollections of actual experiences following Van Manen's (2014) advice to the phenomenological interviewer, "Try to obtain concrete stories of particular situations or events" (p. 317). Through encouraging the wolf educators to share stories and anecdotes about actual experiences, I was able to delve into how certain moments of being a wolf educator had played out. Still, I kept in mind that even if these anecdotes and stories came closer to teasing out actual lived experiences they nonetheless remain recollection, so "already transformations of those experiences" (Van Manen, 2014, p. 313).

Seidman (2013) suggests a three interview structure for interviewing — a first interview focusing on the context and history of the phenomena for the participants, a second on the details of the experience/s, and a final interview on meaning making. While I was only able to interview each participant once, I did incorporate Seidman's three interview structure in some ways as I started with questions related to context, moved on to details about their experiences, and concluded with more reflective meaning-making questions. (See my interview guide in Appendix A.) Further, during the interviews, while I let the discussion flow as much as possible and thus did not ask every question to each participant, I did keep the overall structure suggested by Seidman in mind. According to Seidman (2013), a good in-depth interview is not conducted through strictly adhering to an interview guide due to the fact that the questions asked by the interviewer often evolve during an interview based on the experiences of the 
participant and the ensuing discussion. He states that in-depth interviewing "is designed to ask participants to reconstruct their experience and to explore their meaning. The questions most used in an in-depth interview follow from what the participant has said" (p. 94). I noticed that in some of my earlier interviews, I did stick to my guide more closely, partly due to nervousness and inexperience. However, once I had interviewed a few participants, it became easier to let the interviews travel in various directions and to use the guide more as a reminder to ensure I covered the main topic areas.

I recorded all of the interviews so that I could later transcribe and analyse them. None of the participants had a problem with me recording our discussion and I did obtain their permission and informed written consent prior to each interview. From a phenomenological standpoint, my goal was to come as close as possible to understanding the participants' experiences from their point of view and having a recording of their exact words to go back to was very helpful in this regard (Seidman, 2013).

Of the 16 interviews with 17 participants (which included one double interview), I transcribed ten of them and when I found I was running out of steam and realised that time constraints needed me to speed up the transcription process somewhat, I hired someone to transcribe the other six. Since I made sure there were no identifying features in the recorded interviews, I did not need my transcriber to be added to the list of approved researchers involved in the project. However, just to make sure I was following the appropriate ethical protocols (in case, for example, I missed an identifying feature in the recordings), I gave the individual I hired only interviews where the participants had waived anonymity. Importantly, I reviewed all of the interview transcripts to ensure 
accuracy (see data analysis) and seven of the participants also chose to review their own transcripts to ensure accuracy.

\section{Centre Overviews}

The importance of context, i.e., understanding participants' experiences in connection to a certain place and point in time, is an important element of phenomenological interviewing (Seidman, 2013). To ensure that the interviews I conducted with wolf educators were not without context, I conducted a very brief content analysis of the websites for the centres and/or programs where each worked. I reviewed the programs' stated mission and/or purpose with regards to wolf education, investigated the various types of programs offered/advertised, and reviewed the variety of animals that were involved at each centre/program. I created a short profile for each centre based on this information and later went back to these profiles to generate the centre overviews that appear in chapter four. Because six of the educators chose that they and their centres remain anonymous, I could not create too detailed a description of their centres without revealing identifying features. That being said, while the descriptions thus remain fairly unspecific, they are still useful helping give some context to the educators' experiences and ideas. (Descriptions are in chapter four.)

\section{Observations}

Intending to complement the interviews and program overviews, I visited and made observations at two sites. Observations are sometimes used as a phenomenological method of data collection, and they can be especially useful in helping to "contextualize the phenomenon" (Vagle, 2014, p. 86). Vagle discusses how observations can be recorded in journal entries that include detailed descriptions of what was observed, 
thoughts about what was observed, and perhaps theoretical ponderings. I visited two sites where I observed three programs in total, two at one site and one at the other. (Details about these are provided in chapter four). I also observed one wolf-care session. During these experiences I tried to immerse myself and participate fully and then afterwards I created journal entries about each experience that were rich in detail and included “questions, phrases, [and] wonderings" (Vagle, 2014, p. 86).

From a phenomenological standpoint, I had hoped, as Lichtman (2010) contends, that the observations might help me gain depth of understanding of wolf education. I also hoped to decentre humans in one part of data collection. As Warkentin noted in a jointly authored paper (Oakley et al., 2010), in research on human-animal relations interviews privilege the human perspective over that of the other animal. For this reason, in her own research on humans and whales at aquariums, she intentionally did not conduct any interviews and used observations instead. She found that an observational method allowed her to attend on a more equal plane to both the whales and the humans, as well their interactions. Conducting interviews, she argued, would have "shifted focus away from the immediacy of interacting with whales by enabling a distanced and decontextualized space for talking about them" (Oakley, et al., p. 93).

I concur that my interviews did indeed privilege human perspectives, but I nonetheless needed them in order to answer my research questions. Like Warkentin, however, I also wanted to give voice in some way to the wolves. As I had earlier wondered in a paper on the ethics and possibilities of representing other animals in research, "Because animals do not use human language to speak to us, how can we represent their embodied experiences and our embodied experiences (as animals 
ourselves) with them?" (Kuhl, 2011b, p. 110). Setting out, I believed that being able to observe the wolves and their interaction with human educators in at least a couple of sites might allow the wolves at least some "voice" in my study.

Unfortunately I was unable to observe educator-wolf interaction save at one centre in the midwestern United States. Even so, this one set of observations brought actual wolves into this study in a less abstract way than only hearing about them during the interviews. While a very small part of this research, my observations of wolves during one program and observations of staff interacting with them during a wolf-care session did offer me a different window into understanding the experiences of wolf educators as they work with wolves. (See chapters four and five.)

While observations did open up another avenue to understanding the phenomenon of wolf educators' experiences, I want to acknowledge that as a method it is not without problems. Angrosino (2005) explains that while it has been one of the foundations of fieldwork in social science research, the idea of a neutral, objective observer has been problematized. He writes, “contemporary social and behavioural scientists are increasingly inclined to expect differences in testimony grounded in gender, class, ethnicity, and other factors that are not easy to mix into a consensus" (p. 731). Accordingly, I believe my own positioning and context impacted and coloured what I observed. Nonetheless, I was able to capture a situated, relative type of truth, what Angrosino refers to as "individualized particularism" (p. 742), about the experiences of wolf educators. Or, put in another way, like Russell (2003), I realise that my observations offered me only a "partial perspective." Russell was discussing observations in the context of whalewatching and her line of reasoning is applicable to 
my research looking at wolf education. I like how she builds on the work of Haraway when she says, "my 'situated knowledge' will lead to the development of one particular 'partial perspective' of whalewatching and will contribute one of many voices... who are engaged in discussions about the phenomenon" (p. 127).

\section{Photographs}

Van Manen (2014) suggests that we can "search anywhere in the lifeworld for lived-experience material" (p. 313) including through audio-recorder or camera. Some phenomenological researchers employ a method, often called photo elicitation (Harper, 2002; Vagle, 2014), whereby participants take and/or describe photographs as a means to understanding some aspect of their lived experience (for examples, see: Kirova \& Emme, 2006; Plunkett, Leipert \& Ray, 2013). I had proposed to use this method as another form of data collection, but many of the participants were uninterested or unable to engage in photo elicitation. I therefore decided about two thirds of the way through the interviews not to use this technique. While not interested in engaging in photo elicitation, eight participants still did share photographs and gave me permission to use them as part of my study. Thus instead of photo elicitation, I chose to use photographs as artefacts (Bogdan \& Bicklen, 1998). While eight participants offered photos, the other participants told me that they did not have anything available that they thought would be appropriate, or in a couple cases told me they would share photographs, but in the end never sent them despite me following up with another request. I inserted some of the photographs I did receive into chapters five and six, placing them where they best seemed to represent or correspond to the idea, topic, or theme being discussed. 
Eisner (1997) suggests two (of many) reasons why arts-based forms of representation (including pictures) are useful in educational research that I argue pertain to this study: they can provide a sense of the particularity and/or authenticity of something and they can offer a new or different way of seeing. For me, I believe that through adding participant photos to the text, I have accomplished both to some degree. Rather than just reading about a participant's description of an idea about or an experience with wolves, actual and particular wolves are portrayed. You can see the wolf's eyes and at times you can see the wolf interacting with another wolf or a human. This may allow for another way of understanding in that it offers a visual image of a particular wolf that is less abstract than the text alone.

From a phenomenological standpoint, the photos have also captured a specific moment in time, which according to Seidman (2013) is one of the goals of phenomenological interviewing: to capture the experience or phenomena at a specific place and point in time. Photographs by their very nature add a temporal element to the findings. Finally, from a critical standpoint, I very much wanted to somehow bring the wolves and even the wolves' point of view into this research (Kuhl, 2011b). While I was not able to do this to the degree that I had hoped, the photographs at least offer a glimpse into the lives of the wolves involved in educational efforts in a manner that textual description alone could not have.

\section{Data Analysis}

The interview transcripts were analysed and interpreted using an inductive approach (Seidman, 2013; Thomas, 2006). Seidman suggests that with 
phenomenological interviews, an inductive approach where the researcher "come[s] to the transcripts with an open attitude, seeking what emerges as important and of interest from the text" (p. 119) is preferable to approaching the text in order to test hypotheses or develop theories. Before embarking on this inductive process, I returned to the literature on both inductive analysis and phenomenological approaches. According to both Seidman and Lichtman (2010), there is no one right way to analyse qualitative interview data. In fact, Lichtman notes that "there is less written about the mechanics of doing such analysis than about any other topic in qualitative research" (p. 195). Despite this, my experience coding, categorizing, and developing themes during my Masters study as well as the explanations and examples given by Seidman (2013), Van Manen (2014), Lichtman (2010; 2013), and Thomas (2006) gave me a starting place when I set out to organize and analyse the interviews. The process I describe below would be considered a thematic approach to data analysis and is an approach often taken by those conducting qualitative and/or phenomenological interview research (Lichtman, 2013; Seidman, 2013; Van Manen, 2014).

In discussing the work of Van Manen, Magrini (2012) shares how the analysis phase of pedagogical phenomenology should be about "focusing on identifying and elucidating essential themes within the descriptions gathered" (para. 13) in data collection. In phenomenology, that does not always mean generalizing ideas, it can also mean highlighting some of the uniqueness of human experiences (Magrini, 2012). Keeping this in mind, the next step I took after the interviews were transcribed was to listen to the interviews while reading the transcripts. I did this at least twice for each interview, making myself familiar with the interview content and developing an overall 
familiarity with the elements within the text (Thomas, 2006). The goal in doing so was to get an overall sense of the interviews (Lodico, Spaulding, \& Voegtle, 2006) before focussing on, and highlighting individual pieces.

Next, I embarked on a "selective reading approach" where I began to mark passages that seemed meaningful, "essential or revealing" (Van Manen, 2014, p. 320) while listening to and reading each interview. I then switched to text-only, reading each transcript through fully several more times while creating categories by hand, without the use of software (Seidman, 2013). I noticed early on in this coding process that there were many ideas, anecdotes, and stories that were either common between the educators, or stood out in some way, perhaps because they connected to some piece of literature or simply were poignant and interesting in and of themselves. While my own ideas and biases obviously affected how I interpreted the interviews, as Seidman (2013) suggests, having recordings and transcriptions of the interviews is extremely useful; many times throughout the analysis (and even into the writing process), I returned to the transcripts to ensure I was being as true as possible to the original conversations. From a phenomenological standpoint, it was very helpful to have an accurate account of the participants' exact words to use as a source as I tried to keep in mind the goal of representing their ideas and lived experiences as accurately as I could (Seidman, 2013).

Once I had read the interview transcripts several times, circling passages that seemed meaningful and writing key descriptive words in the left margins of the transcripts, I physically cut up the transcripts, cutting out each quotation or passage where I had marked a descriptive word or phrase in the left margin as well as the participant's initials so I could later remember from whose transcript the passage came. These 
descriptive words or phrases became my categories, or units of meaning (Groenewald, 2004; Seidman, 2013). Next, I listed all the categories on paper and thought about how some might fit together. By doing this, I was able to create a temporary outline with certain categories clumped together under a more general organizational theme (Lichtman, 2013; Seidman, 2013). For example, “creating meaningful experiences” and “educating through busting myths about the wolf" were categorized together under the broader theme "how best to educate." Next I created a label for each category; I started with just over 100 categories but when I noticed some overlap, I was able to reduce this to 98 labels from which I developed the initial outline. (See Table 1 for an example of how sub-themes and themes were developed from the categories). I spread the labels for each category out on a large surface, organizing them using my temporary outline of themes and sub-themes and under each label started to create piles, placing the coded passages from the cut-up transcripts in the appropriate pile. Next, looking through the piles, in some cases I moved the passages and ideas around, for example, if they fit better under a different category. Sometimes two categories became one and the piles merged if they were similar. Other times, if a pile seemed to have a lot of variety within it, I occasionally felt that there were two distinct key ideas or categories going on so I divided them into two piles.

Eventually, I was able to "reduce overlap and redundancy among the categories" (Thomas, 2006, p. 242) as I winnowed them down by finding connections and links between them (Seidman, 2013). Ultimately, through this organization process, I was able to finalize themes and sub-themes. Put fairly simply, themes "identify the major concepts 
Table 1

Example of Theme Development

\begin{tabular}{|c|c|c|c|}
\hline Early Categories & Final Categories & Sub-Themes & $\begin{array}{l}\text { Final } \\
\text { Theme }\end{array}$ \\
\hline $\begin{array}{l}\text { - contact/connection with visitors } \\
\text { - visitors can see wolf behaviours } \\
\text { - visitors see that wolves fear } \\
\text { people or aren't vicious } \\
\text { - visceral, } 5 \text { senses } \\
\text { - people have an emotional } \\
\text { response } \\
\text { - socialized or not? A difference in } \\
\text { messaging }\end{array}$ & $\begin{array}{l}\text { Making connections } \\
\text { A visceral experience } \\
\text { Watching wolf } \\
\text { behaviours } \\
\text { Wolves are not that bad } \\
\text { Wolves as a "carrot" }\end{array}$ & $\begin{array}{l}\text { Experiencing } \\
\text { wolves: } \\
\text { perceived } \\
\text { benefits for } \\
\text { participants }\end{array}$ & \multirow[t]{4}{*}{$\begin{array}{l}\text { Educating } \\
\text { with wolves }\end{array}$} \\
\hline $\begin{array}{l}\text { - puppies very young and socialized } \\
\text { with humans } \\
\text { - wolf on a leash (including not } \\
\text { judging other centres) } \\
\text { - education on the wolf's terms } \\
\text { - education with live wolves is not } \\
\text { entertainment } \\
\text { - understanding wolf behaviour to } \\
\text { keep wolves and visitors safe }\end{array}$ & $\begin{array}{l}\text { Wild or socialized? } \\
\text { Wolf on a leash } \\
\text { Putting wolves first } \\
\text { Ethics of taking people } \\
\text { in with wolves } \\
\text { Other ethical consider. }\end{array}$ & $\begin{array}{l}\text { Wolves in } \\
\text { education: } \\
\text { ethical aspects }\end{array}$ & \\
\hline $\begin{array}{l}\text { - being the voice for captive } \\
\text { wolves/advocate at centre } \\
\text { - The how to's of wolf feeding and } \\
\text { vet care } \\
\text { - What's best for the pack vs. } \\
\text { individual } \\
\text { - Enrichment (toys, fruit, walks, } \\
\text { adventures) } \\
\text { - Committing to an animal for its } \\
\text { whole life } \\
\text { - Wolf retirement } \\
\text { - Naturalized enclosures }\end{array}$ & $\begin{array}{l}\text { Enclosures, packs and } \\
\text { diet } \\
\text { Health care } \\
\text { Committing to wolves } \\
\text { for life } \\
\text { Keeping wolves happy }\end{array}$ & $\begin{array}{l}\text { How to care for } \\
\text { captive wolves }\end{array}$ & \\
\hline $\begin{array}{l}\text { - Best part of job is bonding with } \\
\text { wolves } \\
\text { - Love it when wolves howl } \\
\text { - Difficult when wolves die } \\
\text { - Job is challenging when things are } \\
\text { hard for the wolves } \\
\text { - Stayed in the job for certain } \\
\text { wolves } \\
\text { - Human-wolf trust } \\
\text { - Human-wolf bonds } \\
\text { - Have favourite wolves } \\
\text { - Learning wolf language } \\
\text { - Wolves as individuals vs. species }\end{array}$ & $\begin{array}{l}\text { Relationships are } \\
\text { reciprocal and } \\
\text { individual } \\
\text { Forming bonds } \\
\text { Nurturing } \\
\text { Wolf-educator } \\
\text { communication }\end{array}$ & $\begin{array}{l}\text { Elements of } \\
\text { educator-wolf } \\
\text { relationships }\end{array}$ & \\
\hline
\end{tabular}


p. 307). The themes I developed through this inductive process helped me create "structures of meaning" (Van Manen, 2014, p. 319) to help elucidate educators' experiences with wolves and wolf education.

\section{Limitations}

Arguably, no research can ever be completely objective. With qualitative methods such as interviewing and observation objectivity is even more unlikely given the researcher is immersed in the research process (Seidman, 2013). My own biases are apparent in the very choice of topic and research question, and my participation in the study (through interviewing and observing) will have influenced my findings; who I sought out, the questions I asked, how I asked them, and what I chose to observe shine a brighter light on certain aspects of wolf education. As such, I attempted to openly acknowledge my own stance as I analysed the findings and developed themes and conclusions. While my findings are by no means objective, there are ways in which this study took into account the concepts of validity or trustworthiness and reliability (Seidman, 2013; Van Manen, 2014), namely by interviewing many participants from a variety of programs and comparing and contrasting their stories and experiences (Seidman, 2013), by asking clarifying questions during the interviews, offering participants an opportunity to review transcripts for trustworthiness (which seven of them chose to do), and by using observation and content analysis of centre descriptions along with interviewing (i.e., triangulation) (Cresswell, 2012). For example, in a few instances, during my observations, I witnessed a certain phenomenon and multiple participants discussed that same phenomenon during the interviews. It definitely added strength or 
trustworthiness to those particular findings (for example, the idea of educating through sharing science-based information about wolves or the idea that wolves form social bonds with humans). It also added validity, or trustworthiness, that my research was based on a good phenomenological question, and that continually throughout the interviews I reminded myself that I was seeking to understand "What is this human experience like?" (Van Manen, 2014, p. 350). I believe this led me to asking better questions during the interviews and receiving richer more descriptive accounts on the part of participants.

One limitation of the study related to the interviews was that I interviewed each participant only once, and had developed no familiarity with the participants beforehand. Seidman (2013) recommends a three-interview structure as he believes that can add validity to interview research and Van Manen (2014) discusses the importance of taking the time to develop "a relationship of personal sharing, closeness, or friendliness" (p. 315) before delving into the research topic, whereas I conducted only one interview per participant and before each interview I had no knowledge of, or relationship with my participants as people. In retrospect, multiple interviews with fewer participants may have led to both a more comfortable rapport and richer descriptions about the experience of being a wolf educator.

While the use of telephone and Skype interviewing might be considered a limitation of this study, and I agree there are likely some limiting aspects, Seidman (2013) argues that when necessary, long distance interviews can work. His main point is that if it means the researcher can access appropriate participants who live too far away to make travel and in-person interviews realistic, conducting telephone or Skype interviews is preferable to having to forgo including these individuals. Another issue is that from a 
phenomenological standpoint, I was interested in lived experience, which is always embodied. Interviews over the telephone cannot as readily tap into embodiment as inperson interviews would, but I remind the reader that it is not the interview itself that I was studying. The interview merely served as a tool in which to collect accounts and descriptions of the phenomenon in question, the experience of being a wolf educator (Van Manen, 2014). In fact, there were times when I felt that the telephone interviews worked even better than the Skype interviews where I was face-to-face with my participants as all visual distractions were eliminated and I found myself immersed completely in the participant's stories and accounts.

As with most qualitative research, the findings about the perspectives of the particular wolf educators in this study are not generalizable to all wolf educators but limited to the participants involved; in any case, as noted above, generalizability is not the goal of phenomenological research in the same way as it is with other qualitative empirical methods (Van Manen, 2014).

Finally, while I made some attempt to include the wolves' experiences through observational techniques and photographs, I was not able watch all, or even most, of the participant educators who take part in hands-on wolf care interact with the wolves at their various centres due to a lack of time and resources. Hence, the portion of this study that includes the wolves themselves is only a very small, supplementary part. This limitation troubles me as a researcher interested in disrupting anthropocentrism. Further, and notably, in all but one case, the educators worked with predominantly captive wolves, which is a very particular form of human-wolf experience and interaction. I imagine that 
human-captive wolf relations are unlikely comparable to those that take place between humans and wild wolves.

\section{Ethical Considerations}

The risks (physical, psychological, economic, or social) as outlined by the Tri Council Policy Statement (2010) to the adult educator-participants who took part in this research were minimal. The study was granted approval by Lakehead's Research Ethics Board in May 2014. Prior to the interviews and observations, I gained written, informed consent from all 17 participants (see Appendices B and C). The participants were made aware that participation was voluntary and that they could withdraw from the study at any point in time for any reason without explanation. None, however, chose to withdraw. I also offered them an opportunity to read through the written transcript of their interview and make any changes they wanted. Eight educators chose to either read through their transcripts and/or read through the direct quotations from their interview transcript that I intended to use in writing. Five requested small changes or corrections and/or that I omit something from the transcript from my write-up and I abided by their wishes.

I did explain to the participants that complete participant anonymity might not be possible. For example, if they described unique aspects of the centres or programs where they worked during the interviews, readers familiar with wolf education might be able to identify the program and therefore, quite possibly, the educator. This was pointed out to participants prior to obtaining their consent (both verbally and in the consent letter-see Appendix B) so they did understand the risks. 
Interestingly, despite these various measures to ensure the research was conducted ethically, very quickly into the participant recruitment and interview process, I began to encounter other ethical questions or conundrums. One of the biggest methodological insights I gained through the process of conducting this research is how, ultimately, ethics, at least in the case of a phenomenological interview study such as this, are about relationships. And since relationships are often nuanced and messy, so too are the ethics of undertaking this type of research. I learned that rather than a set of rules to follow, ultimately ethics help act as a guide for "researchers' relationships with their participants" (Seidman, 2013, p. 139). As a case in point, I found that the issue of anonymity was tricky to navigate. As indicated above, I set out initially to offer the participants' anonymity if they so wished. I figured I could maintain their anonymity by using a pseudonym in any writing or reports that arose from the study unless they specifically asked to be identified in the dissertation. I did explain to all of them that they could choose either to remain anonymous or not. However, in one of my early interviews, in confirming verbally with the participant that they wished to remain anonymous, they told me I was incorrect. It turned out that by not writing down a choice of pseudonym in the provided blank on the consent form, they figured they were waiving anonymity and choosing to have me use their name. This actually happened multiple times so I then confirmed verbally what each participating educator preferred. Similarly, several educators, when I attempted to verbally confirm their choice, said "I don't care" or "it doesn't matter" or "you choose." At this point I tried to describe further what the risks might be to having me disclose their name; for example, they might not feel as comfortable sharing anecdotes or stories about their workplace. Eventually, I believe I 
was able to help all of the participants make an informed choice, but clearly the original participant letter was not enough.

Another interesting issue that arose around anonymity was how to discuss the educators' ideas and experiences in relation to where their programs were located if they wished to remain anonymous. Having read Nespor's (2000) paper on the issues of anonymity as it relates to place in qualitative research, and because context is so integral to phenomenological studies (Seidman, 2013; Van Manen, 2014), I did want to be able to make some reference to and explore connections between educator experiences and region if possible, while still protecting educator anonymity. Nespor (2000) worries that without considering the role of specific places in relation to our research, researchers may "unreflectively produce representations of the world that obscure or ignore the connections linking places, writers, participants and readers" (p. 555). This is because the people and relationships we are studying both influence and are influenced by the locations in which they take place. Therefore, "[a]nonymizing a place suggests that the identities and events that happen there float, so to speak, above or outside specific historical and geographical moments" (p. 557).

Taking this into account, after the interview process was over, I reconnected with each educator to see what he or she was most comfortable with in this respect. Some who wished for anonymity were still okay with me discussing their experiences in relation to the general region, province, or state where their centre was located. In other cases, even if an educator was happy with me using their name in the dissertation, they did not want me to name their workplace. Had I not reconnected and discussed the issue with them, I would have assumed otherwise. Eventually, I did try to connect some of my 
findings with the various centres' regions and consequent social-political contexts, but due to the mix of participant desires around personal and program anonymity, I was not able to delve as deeply into this as I would have liked. This, for me, became one of the key limitations of this study.

Ultimately, I came to realise that while having the participants sign forms and read the consent letter was part of the process of conducting an ethical study, more important was that they actually understood and were comfortable with how I went about using their ideas and thoughts around wolves and wolf education. While conducting my analysis and writing up the findings, I often wondered whether I was interpreting and presenting the educators' thoughts and ideas as fairly and accurately as possible. I imagined them sitting down to read the dissertation. Doing so helped me to decide to remove the "ums" and "ahs" and to make minor grammatical corrections in direct quotations as I did not want to make the participant appear less articulate than they were. (Very few of us speak in complete sentences and paragraphs in real life!) This is one example of how I attempted to treat the participants with respect and not merely as a means to an end (Seidman, 2013). I also did so by endeavouring to fully engage with them during the interviews, showing interest in them as people and in their stories and ideas. As well, I strived to consider and treat them as the experts on both their own experiences and ideas and on the topic of wolves and wolf education. Also, as an acknowledgment that their ideas and experiences were integral to the research, I offered to send them either a summary of the research and/or an electronic copy of the whole dissertation once it is complete and defended. Fifteen participating educators requested a summary of the study findings, and ten were interested in the final dissertation. 
In terms of consent for the use of photographs, for the eight educators who offered me photos to use, I had them sign a photo consent form, advising them that people reading the dissertation or papers written from the research might recognize the photos or wolves (see Appendix D), and allowing them to indicate in what capacity I could use the photos (i.e., dissertation, presentations, publications) as well as whether or not I should credit them for the photos.

Finally, after the research is complete, I will store the interview data for five years in the secure storage area in the Faculty of Education at Lakehead University.

\section{Overview of the Following Chapters}

I have divided the findings and discussion of this dissertation into four chapters. Chapter four is a short descriptive chapter that provides a brief overview of the participants as well as the centres and programs where they work. Chapters five through seven focus on the experiences of the participant educators. In each of these chapters (five through seven) I chose to not only present my findings but also discuss them, thereby making connections to relevant research and literature more immediate and avoiding the repetition that would have occurred had I separated them out.

Four overarching themes emerged from analyzing the data and are represented by the titles in chapters five through seven. Chapter five, "The Complexities of Educating for Change," explores the participants' experiences of and beliefs about educating the public about wolves. Chapter six, "Understanding and Working with Wolves," focuses on the participants' beliefs and ideas regarding wolves themselves as well as their experiences interacting with them. In chapter seven, "Engaging in the Controversy of 
Wolf Conservation," I explore how wolf educators engage in and negotiate the controversial subject of wolf conservation. Finally, chapter eight is the concluding chapter in which I summarize the findings in light of my research question and subquestions and point to future research needs. 


\section{CHAPTER FOUR}

\section{PARTICIPANT AND CENTRE OVERVIEWS}

In this chapter I describe both the wolf educators and the centres or programs where they worked. The intent of the chapter is to provide context for understanding the experiences of my participants. The information detailed below is an amalgamation of data gathered through examining and making notes on each centre's website, talking to the educators about the details of their jobs and workplaces (interviews), and through my observations of two programs. This chapter is primarily descriptive and only in a few instances do I make connections to relevant references.

First, I provide information about the participants such as how they got involved in wolf education, some demographic information, and what their jobs entail. I follow this by including some details about the centres and programs where they educate such as their missions/goals, types of programming offered, non-human animals involved, and some general information about the people who visit. This chapter concludes with a description of the observations I conducted at two wolf education sites.

\section{Participant Overview}

I interviewed 17 educators in total, eight women and nine men. Three of the educators were volunteers while the rest were in paid positions, eleven in lead or managerial roles. Four educators who were now in paid positions had started out at their centres/programs as volunteers or interns. The interviewees had been working in wolf education from one to 28 years; 11 had seven or more years experience working as wolf educators. They worked at centres located in a variety of regions throughout North 
America including the Great Lakes region (Ontario, Minnesota, Indiana), the Canadian Rockies in British Columbia, two east coast states (New York and Pennsylvania), one central state (Missouri), the southwestern United States (New Mexico and California), and four northwestern and/or Rocky Mountain states (including Colorado, Washington, Idaho, and one other). See Table 1 for a visual overview of basic demographics.

\section{The Path to Becoming a Wolf Educator}

I asked all the participants how they got started in wolf education, as well as about their experiences with, and beliefs regarding, wolves growing up. I was fascinated to hear so many of them, twelve in total, talk about how they had always liked or loved animals in general or wolves specifically. For example, consider the similarities between the following statements:

Jennifer - I've always been pretty fascinated by animals.

Spencer - I always knew that I wanted to work with animals.

Christina - I always really liked wolves and animals in general.

Rachel - Well, I've loved wolves ever since I can remember.

Matthew - I've been interested in wolves pretty much my whole life, I've had a lifelong interest in them.

Michelle - ... and I always have really liked wolves.

Jeremy - I've always had that interest of wolves since being a teenager.

This interest and affection was a large part of what led them to become wolf educators.

While all of the participants were practicing educators, only one had an academic background in education. More commonly, they had studied science or natural resources; of the fourteen educators who discussed their educational background, twelve talked about studying science or natural resources in college or university. While only one participant had studied education, six had gained work experience in education prior to becoming a wolf educator, three had worked at environmental education programs or summer camps, one received educational training as a docent, one had worked as a park 
interpreter, and one had worked as an educational aide in a classroom). For most, it was an interest in animals, nature, and/or wolves that drew them to the work rather than a desire to become an educator in an informal or formal setting.

Mony and Heimlich (2008) interviewed and observed docents and visitors at a zoo in the Midwestern United States and also discovered that a love for animals was a key motivator for the docents working there: "The primary motivations described by docents...were a love for animals and an intention to protect these animals in the wild through educating visitors" (p. 156). Taylor (2009) suggests that it is probably very common in wolf education that educators lack formal training and that often these programs are "run by people passionate about conservation, but who have no formal training or experience as teachers or educators" (p. 170).

When it came to experience working with animals, nine of the educators reported having worked with animals prior to becoming wolf educators. Of these, three had grown up on farms, three had worked or volunteered at zoos, one grew up working with horses and leading trail rides, one had worked at an animal shelter, one had interned at a wildlife rescue facility, and one had volunteered at a raptor rehabilitation facility. With regards to personal experiences with wolves prior to their present work, most (13) had not grown up in areas with wild wolves. Of the four who did grow up in regions inhabited by wild wolves, only two mentioned having encountered wolves when they were younger. However, five educators did talk about experiencing an educational program involving wolves when they were youths. 
Table 2

Participant Overview

\begin{tabular}{|c|c|c|c|c|c|}
\hline $\begin{array}{l}\text { Name or } \\
\text { Pseudonym }\end{array}$ & $\begin{array}{l}\text { Male/ } \\
\text { Female }\end{array}$ & Position & $\begin{array}{l}\text { \# of Years } \\
\text { as Wolf } \\
\text { Educator }\end{array}$ & $\begin{array}{l}\text { Education } \\
\text { Background }\end{array}$ & $\begin{array}{l}\text { Region of } \\
\text { Centre/Program }\end{array}$ \\
\hline Rick & Male & $\begin{array}{l}\text { Senior Park } \\
\text { Naturalist }\end{array}$ & 17 & $\begin{array}{l}\text { BSc Biology, } \\
\text { Bachelor of } \\
\text { Education }\end{array}$ & Ontario \\
\hline Shelley & Female & $\begin{array}{l}\text { Owner/ } \\
\text { Operator }\end{array}$ & 13 & Unknown & $\begin{array}{l}\text { British } \\
\text { Columbia }\end{array}$ \\
\hline Jennifer & Female & Educator & 1 & $\begin{array}{l}\text { Ecosystem } \\
\text { Management, } \\
\text { Urban Forestry, } \\
\text { Communications }\end{array}$ & $\begin{array}{l}\text { Withheld at } \\
\text { participant } \\
\text { request }\end{array}$ \\
\hline Noel & Male & Wolf Specialist & 1 & BSc Biology & Minnesota \\
\hline Monique & Female & Wolf Curator & 28 & $\begin{array}{l}\text { Bachelor of } \\
\text { Science \& Natural } \\
\text { Resources, Masters } \\
\text { Forestry }\end{array}$ & Minnesota \\
\hline Denny & Male & Volunteer & 8 & Unknown & Pennsylvania \\
\hline Spencer & Male & $\begin{array}{l}\text { Operations } \\
\text { Manager }\end{array}$ & 9 & BSc. Zoology & New York \\
\hline Ashley & Female & $\begin{array}{l}\text { Director of } \\
\text { Education }\end{array}$ & $11 / 2$ & $\begin{array}{l}\text { BA } \\
\text { Communications, } \\
\text { Juris Dr. Law }\end{array}$ & Missouri \\
\hline Leyton & Male & $\begin{array}{l}\text { Executive } \\
\text { Director }\end{array}$ & 18 & Unknown & New Mexico \\
\hline Michelle & Female & Volunteer & $21 / 2$ & Zoology & Colorado \\
\hline Rachel & Female & $\begin{array}{l}\text { Lead Educator, } \\
\text { Volunteer }\end{array}$ & 1 & $\begin{array}{l}\text { Masters in Ecology } \\
\text { and Evolutionary } \\
\text { Biology }\end{array}$ & Colorado \\
\hline Monty & Male & $\begin{array}{l}\text { Senior Wolf } \\
\text { Handler, } \\
\text { Resident } \\
\text { Photographer }\end{array}$ & 26 & BSc. Earth Science & Indiana \\
\hline Jeremy & Male & Site Manager & 16 & $\begin{array}{l}\text { BSc. } \\
\text { Environmental } \\
\text { Biology }\end{array}$ & Idaho \\
\hline Gwen & Female & $\begin{array}{l}\text { Education } \\
\text { Curator }\end{array}$ & 9 & $\begin{array}{l}\text { Natural Resource } \\
\text { Recreation and } \\
\text { Tourism }\end{array}$ & $\begin{array}{l}\text { Northwestern } \\
\text { United States }\end{array}$ \\
\hline Matthew & Male & $\begin{array}{l}\text { Education } \\
\text { Program } \\
\text { Coordinator }\end{array}$ & 7 & $\begin{array}{l}\text { Bachelor of History } \\
\text { and Philosophy }\end{array}$ & $\begin{array}{l}\text { Northwestern } \\
\text { United States }\end{array}$ \\
\hline Erik & Male & $\begin{array}{l}\text { Education } \\
\text { Director and } \\
\text { Animal Care }\end{array}$ & 9 & Science & Washington \\
\hline Christina & Female & $\begin{array}{l}\text { Program } \\
\text { Coordinator }\end{array}$ & 2 & BSc. Science & California \\
\hline
\end{tabular}




\section{What Wolf Educators Do: Tasks}

I asked all of the educators what their jobs or volunteer positions involved. In this chapter, I provide only a brief summary of their typical tasks; richer details will appear in later chapters. While I identify the number of educators who talked about a certain task, there may be others who in fact take part in a particular task but failed to mention it in their interview. However, even if the tally is off by one or two, the following overview gives the reader an idea of what being a wolf educator involves.

Obviously, all of the interviewees had as a primary task educating people about wolves as it was one of the requirements to take part in the study. Seven described some of their work as informal education; for example, some centres post an educator by the wolf viewing area to answer visitor questions as they arise. Some centres offer both formal and informal programs and 16 of the 17 educators reported delivering formal programs such as presentations about wolf ecology for school groups. Eight educators also discussed offering off-site educational programs, for instance, visiting a nature centre or school.

Eleven educators were in lead or managerial positions, consequently seven of this group talked about training and/or managing staff, volunteers, or interns. Ten educators also mentioned carrying out administrative tasks such as answering and returning phone calls, budgetary management, booking groups, and maintaining websites or Facebook pages. I also heard how seven educators were involved in creating curriculum for their centre's programs, sometimes in line with the particular state or province's education curriculum (for example, a science or social studies lesson for sixth graders). 
Eleven of the educators also discussed taking part in wolf care at their respective centres. This involved feeding, cleaning enclosures, maintaining social bonds with the wolves, and health care. Four of these educators also discussed being involved with training wolves, sometimes for research purposes, other times so that the wolves would be able to tolerate interacting with visitors or travelling off-site. Closely tied to wolf care, three educators talked about doing site maintenance, such as repairing or building wolf enclosures.

Finally, six educators discussed being involved with wolf research projects. In fact, three educators got involved with wolf education initially through being connected to particular wolf research projects. These projects included work with wild and captive wolves on research like comparing dog and wolf behaviour, how wolves mark territory, and training dogs to track wolves.

\section{Centre/Program Overviews}

The 17 educators I interviewed worked in 15 different wolf centres or programs around North America, representing 13 different states or provinces (see Table 1). Because seven of them requested anonymity for their centres, for the most part I will only share details about the centres in general terms to give the educators' experiences context without disclosing too much about the actual centres to allow for identification. Where I do reveal the names of an organization, I have only done so where the participants waived anonymity. Below, I provide an overview of the centres including their missions, designation, types of programs offered and animals involved, and an overview of the visitors they serve. The information was collected through talking to the participants 
about their centres and programs, reviewing the centre's and program's websites, examining printed literature (in three cases), and visiting two sites.

\section{Missions and Goals ${ }^{2}$}

The four main categories that show up in the various titles, missions, and goals are: education, research, conservation, and rescue or sanctuary. First, all 15 of the centres/programs have education somewhere in their title, mission, or stated goals, which makes sense since it was one of the things I looked for when recruiting participants. For eight of the centres/programs, education is their main mission. For example, Colorado Wolf and Wildlife Center states part of their main mission is to: "Educate the public through tours and programs about the importance of Wolves, Coyotes, and Foxes to our eco-system" ("Our Mission Statement”, n.d., para. 1). Alternatively, in five other cases, education is stated as a means to accomplishing the centre or program's mission of conservation or recovery. For example, The Wolf Conservation Center (WCC) in New York states their mission as such: "to promote wolf conservation by teaching about wolves, their relationship to the environment, and the human role in protecting their future" ("About Us," 2015, para. 2). In a couple of other cases, the educational part of the mission is more vague, with statements such as "an opportunity to observe, understand and appreciate" animals.

Wolf conservation objectives (sometimes worded as protection, sustainability, or survival) show up in the names, missions, or goals of ten of the 15 centres/programs. Five facilities or programs either have research or science as part of their name/title or

${ }^{2}$ While admittedly slightly awkward, I wrote this small overview of the wolf centres and their programming in the present tense despite the fact that the rest of the dissertation is written in the past tense. It felt incorrect to write how the centres "educated" or "offered" programs in the past tense when, in fact, they still are doing this work. 
declare it as one of their main aims. For instance, Wolf Park in Indiana is "dedicated to behavioural research, education, and conservation, with the objective of improving the public's understanding of wolves and the value they provide to our environment" (“Mission,” 2015, para.1).

Finally, six of the organizations are dedicated to rescue or sanctuary for wolves (and sometimes wolf hybrids). For example, Wild Spirit Wolf Sanctuary in New Mexico states that they "rescue displaced, unwanted, and un-releasable captive-bred wolves, wolf-dogs, and other related species" (“Our Mission,” n.d., para. 1).

\section{Organizations' Details}

Twelve of the centres/programs are not-for profits with a designation as 501(c) $3^{3}$ in the United States, four of which are also involved in the Species Survival Plan (SSP), which is defined particularly nicely on Wolf Haven's website (2014):

A Species Survival Plan (SSP) is a cooperative effort between the U.S. Fish and Wildlife Service (USFWS) and the Association of Zoos and Aquariums (AZA), as well as non-AZA participants (like Wolf Haven). An SSP program is designed to oversee the population management of an endangered species in captivity and to enhance their conservation in the wild. ("Conservation," para. 2)

Of these four centres, three are involved in the Red Wolf SSP and all four are part of the Mexican Grey Wolf SSP. Besides being not-for-profits, two centres are also accredited by the Association for Zoos and Aquariums (AZA). Of the remaining three centres/programs, two are private organizations designed to generate a profit and one is a program offered annually in Algonquin Provincial Park, which is part of the Ontario Provincial Parks system and administered by the Ministry of Natural Resources, a provincial government agency.

${ }^{3}$ A 501(c)3 is an IRS tax designation for non-profit charities. 


\section{Programming}

The following is meant to give the reader a sense of the programming that takes place at the various centres. The information was collected from the centres' websites. However, I did notice that sometimes the educators talked about a type of programming during an interview (e.g., visiting a school to present a program) that wasn't advertised on the centre's website, so while I expect the following gives an adequate impression of the various programs that take place presently, the numbers may be slightly off, by one or two, in a few instances.

Onsite programming. Fourteen of the 15 centres/programs have at least some formal educational programming (e.g., interpretive talks, seminars) and in at least six cases, all visitors can only see wolves with a guide/educator present. Alternatively, at five centres, visitors can self-guide or view wolves informally. Typical onsite programming includes an interpretive talk about wolves along with a tour to view wolves. As well, there are specialty tours and events held at some centres; for example, tours that include viewing wolves feeding are offered by four of the centres. Likewise, nine centres offer some type of photography workshop or session, and nine centres advertise various events, such as fundraisers with special programming. Full moon events are popular and often include howling; eight centres/programs have some type of full moon and/or howling event. Thirteen centres advertise programming for groups (e.g., Scout groups, school groups), and five offer day camps for children. Finally, five centres offer lectures or seminars with more in-depth educational content about wolves.

Offsite programs. Seven of the centres advertise offsite programs without a wolf present; for example, they will come and do a talk about wolves for a school class or an 
organization. Five of the centres offer offsite programs that involve bringing an "ambassador" wolf or wolf hybrid along. Three centres offer (or had offered in the past) some type of tourism activity such as trips to Yellowstone or the Arctic to learn about and view wolves. Finally, three programs offer field trips to howl with wild wolves (as opposed to doing so with captive wolves onsite).

Meet-a-wolf programs. Seven centres offer opportunities for visitors to interact with wolves, in most cases through signing up for specialty programming (rather than the typical experience offered to general visitors). However, two centres do offer opportunities for general visitors to meet and interact with wolves. Both of these centres do so through interactive programming at offsite talks that include a socialized ambassador wolf. One also allows centre visitors to enter an enclosure with a staff member and a socialized pack of wolves. Five other centres also offer specialty programs where visitors can meet a wolf; for example, two centres have a "take a walk with a wolf" activity and one centre allows funders/sponsors to interact with the wolves that they sponsor once a year; and finally, two centres offer "keeper for a day" opportunities whereby visitors can help staff with tasks such as feeding, training, and cleaning enclosures.

Educating with technology. Seven of the educators I interviewed talked about using technology of various sorts in their educational efforts. According to the various websites, four offer some type of distance learning programming. For example, Erik discussed a "Skype in the Classroom" program where teachers can contact the centre to request a guest lesson. He explained, "And so we do 30 minute Skypes with kids all across the world...we've done all the way from Hong Kong, all the way to Europe." Six 
centres use live webcams so that people from all over the world can observe the centres' wolves. Spencer, for example, explained the attraction of the temporal nature of webcams: 'it's different looking at a video versus a webcam that's live, because it's happening, you know, right now."

Other. A variety of other activities are offered at the various centres. For example, ten of the centres provide information on wolf biology and ecology on their websites, and three centres also make curriculum available for teachers online. Finally, twelve of the 15 programs/centres advertise some type of volunteer and/or internship program on their websites.

\section{Animals}

For all of the centres/programs, wolves are either the only, or one of the key, focal species. These wolves include one or more of Grey wolves (Canis, lupus), Mexican Grey wolves (Canis lupus baileyi), and Red wolves (Canis rufus). Five of the centres/programs focus on wolves only. The other ten centres have wolves alongside other species, most often wolf hybrids or other canines such as coyotes or foxes. For example, five centres have wolf-dog hybrids on site as part of their sanctuary and/or rescue mission. Five centres have foxes, four centres have coyotes, and two centres have other canines (e.g., African painted dogs, maned wolves, dingoes and/or New Guinea singing dogs.) As well, three of the centres have non-canine species, but for the purpose of anonymity, I will not list the other species here.

The number of animals on site ranges from five wolves to over 60 canines (including wolf-dog hybrids and some other canine species). Probably because of the nature of housing such animals, all of the centres/programs are located in semi-rural or 
rural areas. For the most part, the enclosures for the wolves and other canines are naturally vegetated, and sometimes the enclosures are quite large. For example, some educators described enclosures that were a half-acre per two animals while others discussed packs that had enclosures as large as seven, 15, or 20 acres.

\section{Visitors}

It was evident to me from both reading the literature on wolf education and talking to the educators that wolf-focused tourism is popular in North America (Fritts, et al., 2003; Troxell et al., 2009). For example, according to one of my participants, Rick, a single wolf-howl program in Algonquin Provincial Park attracts, on average, 1,600 visitors. Likewise, Denny told me that on a morning weekend at the Wolf Sanctuary of Pennsylvania, over 200 people will sometimes show up for a morning tour. Likewise, two educators told me their centres see over 20,000 visitors a year and another educator told me they have upwards of 35,000 to 40,000 guests each year.

The centres and programs serve a wide variety of visitors. While some seem to cater more to people in nearby regions, others attract visitors who are travelling in the area, and many of the centres are marketed online as local tourist attractions on, for example, Yelp or Trip Advisor. Sometimes their tourism appeal is bolstered by their proximity to parks; at least seven of the educators worked at centres/programs located close to large national and/or provincial parks or wilderness areas. As a case in point, Shelley at Northern Lights Wolf Centre suggested that some of her centre's tourist appeal is their proximity to Banff National Park. She explained that they serve a large number of European and other international visitors, so much so that they have their basic talk 
translated on handouts in different languages. She estimated that only one or two percent of the visitors they serve are local.

The centres cater to a wide age range. As can be seen by some of the program descriptions above, some centres offer both general programming as well as age-specific programming such as wine and cheese events for adults, campfire events catering to families, and day camps and field trip programs for different ages of children.

Visitor attitudes. Finally, most (13) of the educators felt that those who visit their respective centres/programs already have positive or at least neutral attitudes towards wolves. A study that looked at visitor attitudes at one wolf centre supports this observation (Black \& Rutberg, 2007) as do more general studies that found that people who visit conservation themed exhibits, including zoos and aquariums, already tend to have positive attitudes towards, and/or knowledge about, conservation (Ballantyne, Packer, Hughes \& Dierking, 2007; Falk, Reinhard, Vernon, Bronnenkant, Heimlich \& Deans, 2007; Mony \& Heimlich, 2008). That being said, four educators discussed frequent encounters with visitors who have negative perceptions or attitudes about wolves.

I suspect that centre locations and whether or not the centres have other species onsite might have an impact on what type of visitors are attracted to specific sites. For example, participants who worked at centres and programs that were located in regions where wild wolves were part of the landscape were more likely to mention interactions with participants who felt negatively about wolves. As well, four educators suggested that because there were no wolves on the landscape in their region, visitors typically had no experience of human-wolf conflict and therefore felt positively towards them. For 
example, Monty stated: “So, it's a really simple formula. If there haven't been wolves in an area and there's not talk of wolf recovery - wolves are listed as extinct in Indiana, the state doesn't want wolves, it really doesn't have good habitat to put wolves, wilderness areas — so there's not talk of wolf recovery here and because of that everyone is very supportive of wolf recovery." Similarly, Ashley said, "You know, people who don't have a stake in it, if we're talking about wolves in Missouri, there are not wolves in Missouri. People don't care right now, 'Yeah yeah, they're great, whatever,' for the most part."

Finally, while the educators stated that most visitors who come to the centres and programs have positive attitudes towards wolves, five of the 13 educators who felt their guests were primarily positive towards wolves also told me about the occasional visitor with negative attitudes who got dragged along by a family or a group. For example, Jeremy said, "when we get a true anti-wolf person here, it's because they're with a family or group of people that want to be here and they just are sort of tagging along and they don't really want to be here."

\section{Program Observations}

I was able to conduct observations at two wolf education sites in the summer of 2014. The first site I observed was in Algonquin Provincial Park. Each summer they offer a wolf howling program that runs every Thursday in August. Unfortunately, park staff were unable to locate a pack to take the public to howl with on the week that I was there so the program did not involve a field trip to howl with wild wolves but rather an interpretive talk and slide show at the park's visitor centre. The talk was given by one of my participants, Rick. 
The second site was in the midwestern United States where I observed two formal programs and a wolf care session. One program focused on the diet and feeding habits of wolves and included an interpretive talk with slides followed by viewing the centre's wolves being fed beaver carcasses. The second program was an interpretive talk also using slides on wolf communication, followed by a field trip in the local area to attempt howling with wild wolves. Unfortunately, both of these programs were delivered by intern educators rather than study participants. Still, observing both of these programs gave me an idea about the content and delivery of education being offered at that centre.

\section{Algonquin wolf talk: Content and delivery. The Algonquin presentation} covered a wide variety of topics related to the wolf, focusing mostly on the wolves of Algonquin Provincial Park. The talk included the following: wolf biology and ecology, including the type of wolf found in the park, diet, denning, puppies, pack structure, and communication; the history of wolf protection in the park from being poisoned, hunted, and trapped by rangers to protected; research, including the initial study in the area by Dr. Pimlott as well as information about collaring and tracking; human-wolf relations and attitudes over decades, including possible roots of negativity such as competition with humans (hunters, farmers) and various cultural stories; and finally, information about the wolf program itself, both its history and logistics. The slides complemented the talk and offered nice visuals for each topic being discussed.

The Algonquin program was delivered by the senior park naturalist, Rick Stronks, who has 17 years of experience delivering the wolf howling program each summer. Here is a short excerpt from my notes from my observations about the manner in which the presentation was delivered: 
Rick is an animated speaker, and he has obviously done this talk many times. His enthusiasm for the subject shows through, and he uses humour to connect with people. He also talks not only about wolves, but about the history of wolves in the park, including the early hunting and trapping efforts by rangers, the research that has taken place, and the introduction of the wolf howling program including the logistics involved. My sense, as an audience member, is that you are an intelligent person, an insider, and he's sharing not only biology and ecology with you, but the details of what it means to do a large public wolf howl. He does not talk down to the audience.

While his style was enthusiastic and engaging, I was intrigued by the somewhat neutral manner in which the information was delivered. At times, it was clear he was offering differing perspectives and attempting to do so in an unbiased manner. For example, when examining some of the roots of negative attitudes towards wolves, like hunters disliking them because of competition for prey, he stated, "You can understand that perspective." Similarly, when discussing predation on livestock he iterated, "You could understand a farmer not wanting livestock taken by wolves." This was a common approach throughout his presentation whether he was talking about how early researchers in 1964 and 1965 had the wolves protected in the park for the duration of their study then killed over a 100 of them to complete their study, or when he was discussing how park rangers in the 1920s and 1930s trapped, hunted, and poisoned wolves and got to keep the bounties. Through the use of poison, they killed all sorts of other animals in the process as well. While he shared how it is unlikely that these practices would take place today given both contemporary attitudes towards wildlife and today's research practices, he did not suggest one approach or perspective was better than the other but rather simply pointed out the contrast.

The only clear advocacy message he offered was how lucky Ontarians are to have large protected spaces with intact ecosystems in the province. Also, in pondering out 
loud why the wolf howling program has drawn so many people since its inception, he discussed a need people may have in today's highly urbanized culture to connect with nature. He suggested that hearing wolves howl was one of those sounds that help people feel this connection.

Programs at a midwestern United States centre: Content and delivery. The first program I observed was the wolf-feeding program. It is presented in a large windowed theatre that looks out over the wolf enclosure. A ledge runs along the viewing window and the two screens where the slide show is displayed are in front of this ledge. The intern educator used some props - a moose leg, a cast of a wolf print, and a wolf skull - which he passed around during the talk. About 25 people attended the session, including 5 children who mostly sat on the ledge looking out at the wolves rather than viewing the screens.

The presentation was fairly simple and focused on the hunting techniques and diet of wild wolves. The content was predominantly focused on scientific facts about wolves. For example, food sources for midwestern wolves were discussed (e.g., the percentage of deer, moose, beaver in their diet), prey techniques, and survival adaptations for both wolves and their prey. Besides a short comment about wolves preying on livestock (i.e., how it can be an issue, but makes up a very small portion of their diet), human-wolf relations were barely touched on. I personally found it hard to concentrate on the talk because the wolves in the enclosure were active, interesting, and distracting. They were playing and running around, energized perhaps by the coming feeding.

My impression with the delivery was that the audience was assumed to be completely ignorant about wolves. I also found it interesting that the talk focused on the 
diet and prey of wild wolves but there was nothing in the talk about the actual wolves we were going to watch eat. Where did the beaver carcasses come from, for example? Why were the wolves we were watching so excited and active?

After the talk, a young woman entered the enclosure with a wheelbarrow containing 4 beavers (or, rather, pieces of beaver). Two educators walked around amongst the visitors while we gathered close to the windows to watch the wolves eat. These two educators informally described some of the social and pack behaviours we were observing. To me, watching the wolves interact while they ate was one of the more interesting things about the program and I felt that the educators might have done a better job of talking about this during the formal presentation.

Communication program. The second program I attended and observed was one focused on wolf communication. It took place in the evening and included a presentation in the theatre room followed by a drive in the facility's bus to a spot bordering the local wilderness area. Led by two educators, we (me and eight other participants) howled, hoping to get a response from wild wolves.

The talk was very short and delivered by a new intern educator who was giving it for the first time. It appeared as if she had not run through the slide show and talk before having to present it. She could not figure out how to get the audio for the recorded howls to play (which would demonstrate three types of howls). This flustered her. It also was obvious she was unfamiliar with the content of the talk, which included types and aspects of wolf communication (e.g., olfactory, vocalizations, postures). Like the wolf feeding program at this centre, the information was predominantly biological facts about wolves. 
After the talk, we drove to three spots on a gravel road bordering the local wilderness area and howled. We did get a response at one site, but through my own personal local knowledge, I knew that our "answer" was from a group of sled dogs (huskies) rather than wolves. Even though the talk part of this presentation was poorly delivered, the experiential aspect of going out at night to howl with wild wolves seemed to be a highlight for the other visitors. There was no indication that either the educators or the participants knew we were howling with huskies rather than wolves, and I did not mention it given my research role.

\section{Conclusion}

The goal of this chapter was to provide context for understanding the experiences of wolf educators, which I will explore in the three chapters that follow. Some of the information above such as the number of educators who cited a love and/or interest in animals as a motivating factor in leading them to become wolf educators, the number of educators who had educational backgrounds in science, or the amount of science-based information I observed at three programs foreshadow some of the findings I will discuss later. On that note, I now turn in the next chapter to my research question, "What are the experiences of educators who work at programs that include live wolves?" After poring over the interview transcripts, reading through my journals from my observations at two programs, and considering the photos eight of my participants shared with me, key themes about what it meant to be a wolf educator emerged. 


\section{CHAPTER FIVE}

\section{THE COMPLEXITIES OF EDUCATING FOR CHANGE}

The first big theme that emerged from the interviews focused on participants' ideas about, and experiences of, wolf education or "The Complexities of Educating for Change." This chapter is organized under three sub-themes: what educators were hoping to accomplish through wolf education (the purpose); what they believed were the best approaches and methods for educating visitors; and what they found most rewarding and challenging about being a wolf educator. In this chapter, and the two that follow, I generally organize the categories under each sub-theme from most commonly discussed to least. However, in some instances I organize them in the manner that they best flow (for example if the subject of two categories is closely connected), and therefore enable better phenomenological understanding or reflection (Van Manen, 2014).

\section{What Kind of Change? The Purpose of Wolf Education}

As discussed in chapter four, each centre and program declared certain missions and goals, but I was most interested in what exactly the educators felt the purpose of their work was. What were the key messages they were hoping participants/visitors were taking away with them? What were they ultimately hoping to accomplish through teaching about wolves? From a phenomenological perspective, I wanted to explore their experiences of educating about wolves as well as uncover why they felt it was so important. After analysing the interviews, I found two common purposes emerged: the importance of wild wolf conservation, and spreading the message that "wolves don't make good pets." While there were some other less common ideas about the purpose of 
wolf education such as instilling a connection with nature and wildlife, helping people overcome negativity about and fear of wolves, and busting myths about the wolf, these were so often cited again under the next sub-theme, "how best to educate," that to avoid redundancy, I decided to discuss them there instead, where I felt they fit slightly better.

\section{Conservation of Wild Wolves}

All 17 of the educators discussed being advocates for wolf conservation or survival on some level. While they differed on how best to educate for wolf conservation or what the content for wolf conservation education should be (which I discuss in more depth in the section that follows), all of them stated that conservation was one of their key goals. This is not surprising given that wolf education programs have often been part of conservation and reintroduction plans as a means to promote residents' understanding and tolerance of the species (Fritts, et al., 2003; Troxell et al., 2009). Thirteen educators zeroed in on the importance of wolf conservation for healthy ecosystems, and six also explained how conserving the wolf as part of healthy ecosystems ultimately affects human health as well.

Wolves' ecological importance. Matthew, for one, described how part of the message he wants to share is the wolf's importance in functioning ecosystems. He explained:

I think what we need to do is, we need to place predators in the context of the larger ecosystem. Basically saying, "Here's where they fit in, here's the role they play, here's the effect that they have when they're removed from the ecosystem." And let people understand that ecosystems are healthier with a full complement of component species. All the species play a vital role. But some actually play a larger, and more important role than others. And really, the most important are keystone species, are these top predators, these apex predators. And so we want people to know that, and understand that. That if you take wolves away and suddenly the elk and deer populations are up, and they have an effect on the plant population, and there's a collapse of ... aspen and willow and cottonwood trees, 
and pretty soon the amphibian populations are affected and things like that. So lately, we've definitely been emphasizing the vital roles that [wolves] play, and talking about trophic cascades a lot.

Also speaking about the importance of conservation, Rick pointed out that wolf conservation requires protection of habitat that is suitable for wolves. He explained how the wolf howling program in Algonquin Park, where participants learn about the importance of wolves, consequently includes a message about the importance of wildlands and protected spaces:

I also think that part of the messaging here is about the importance of large protected spaces. We are so lucky in Ontario, and in Canada that we have had people in our history that have set aside large tracts of land for protective spaces. Because we know that you have to protect a big chunk of ecosystem in order to have a species like wolves protected.

Wolf conservation was not only a goal of educators in places where wolves still

roam. Spencer works at a centre in New York state where wolves have long been extinct, yet he felt that educating people about wolves might help humans tolerate their existence, and therefore conservation, if they ever returned or were reintroduced in the region. He said, "You know, I think what we're doing is giving wolves an avenue, more of an avenue to even get here, or people to think about wolves being around here. And I think it would definitely help their survival, well I know it would help their survival if [the wolves] did make it here."

Wolf conservation is important for humans. Six educators discussed the importance of wolves, and that not only ecosystems but also humans might ultimately be impacted by the absence of wolves. For example, Leyton said, "If we decide as a species to destroy another species, we're only destroying ourselves." Shelley, too, felt that many 
people have lost touch with how a healthy environment is connected to human health.

She believed teaching about wolves could aid people in making that connection:

My mission is just to create a general awareness about the species. And the purpose, I think the reason we need to do it, is then we'll understand ourselves, and our whole outlook on the way ecology and the fundamentals of the forest system work. And I think that's why we need to do it. Because I don't think people understand how having trees keeps us alive and how having water keeps us alive... understanding the connections, that you need to have the predator there, you need to have an ant, you need to have all these things to help maintain [systems].

\section{Wolves Don't Make Good Pets}

While all of the educators discussed the goal of conservation, the second most common educational goal, discussed by seven of the educators, was wanting to get out the message that wolves do not make good pets. Five of these educators worked at centres that rescue wolves and/or wolf-dog hybrids and helping people understand that wolves make unsuitable pets was, in fact, a key mission of the centres. However, two educators who also discussed this message worked at centres not involved in wolf rescue but nonetheless were well acquainted with the problems of wolf-dog and/or wolf ownership. For example, Ashley explained how wolf-dog pets are popular in her region and her centre often gets phone calls from owners when they find out these animals are difficult to manage. She expounded, "We try to educate the best we can about that topic. And you know, that's totally separate from the reintroduction and endangered species side of things, but it's still a wolf issue. Wolves are not meant to live in people's houses."

The five educators who worked at programs that did some form of rescue talked most extensively about the issues of wolf and wolf-dog ownership and their aim in educating people about the issue. I learned that there are many wolves and part wolves 
living as pets, at least in the United States. Often, commonly around sexual maturity, the animals become too much to handle. Leyton said, "By two years old, people have discovered that this is not going to act like a dog. It's destroying their life. New life adventures pop up and they have to get rid of their so-called pet." Likewise, Rachel explained that, “90\% of the time it doesn't work out with the animals for one reason or another, they can't be adopted out to a new family, and they have to either be euthanized or they have to be sent to a facility like ours... We get calls every week, a few times, like, 'Hey can you take my wolf?'” Rachel went on to describe how most rescue facilities are full and the majority of these animals end up having to be euthanized: "[T]his statistic has probably changed since I learned it, but I think it's something like 2,000 wolf-dogs are put down every year, only to be replaced by 2,000 more." She continued, "Something like 6,000 [wild] wolves are in the United States and over a quarter million are living in captivity."

Three educators argued that educating about the pitfalls of wolf and wolf-dog ownership also included a message that wolves should be wild, not domestic. Michelle said, "We advocate for wolves in the wild, not in the backyard." Similarly, Leyton stated, "No wild animal's a pet. They don't want to be your pet" and that a wolf is "not for your house or your backyard, your basement, your garage, your condominium, it's for the wild."

\section{The Purpose of Wolf Education: Discussion Points}

As I pondered the two most common goals discussed by these educators, conservation and discouragement of wolves as pets, I turned back to the research literature. Since the 1970 s, coinciding with increased public consciousness of 
environmental issues, many zoos and aquariums have changed their branding to emphasize species conversation and education rather than entertainment (Marino, Lilienfeld, Malamud, Nobis, \& Broglio, 2010). While not all of the facilities where my participants worked could be considered zoos, 14 of the 15 programs do house captive wolves so research about zoo education seems applicable here. As noted, conservation messaging was clearly important to my participants. Similarly, Mony and Heimlich (2008) found docents at a zoo in the midwestern United States believed animal conservation was an important part of their messaging. Interestingly, when Mony and Heimlich actually observed the docents in action, they discovered that they were, in fact, communicating little conservation content but instead were mostly conveying "facts" such as the animals' age, weight, diet, and behaviours. Based on what my participants reported in the interviews, I suspect there is more variety amongst my participants than Mony and Heimlich's group, with some sharing mostly facts about wolves while others are placing more emphasis on conservation messages alongside scientific information. However, I was not able to observe these educators and thus cannot make any firm conclusions about what actually takes place in this regard.

For the six educators who discussed how the health of other species also affects humans, a more complex conservation message was emphasized, even if their intent in making such connections was understood as an attempt to get people to care. Some ecologists, environmental educators, and philosophers also emphasize the importance of understanding the interconnections between the health of humans and other species (e.g., Bekoff, 2007; Capra, 2007; Humes, 2008; Lynn, 2002; Oakley et al., 2010; Selby, 1995). As Bekoff (2007) argued in a chapter looking at human anthropogenic effects on animals, 
"the deep reciprocal interconnections among members of the earth community are such that we are all in this together" (pp. 912-913). Others, however, have expressed concern that messaging that emphasizes human health rather than the intrinsic value of other life reinforces anthropocentrism (Livingston, 1981; Selby, 2000).

Finally, the message that wolves do not make good pets is not something I have seen discussed much in the academic literature. Certainly, there is a fair bit written in animal welfare circles about the millions of unwanted healthy cats and dogs that are euthanized each year (e.g., Case, 2008; "Pets By the Numbers," 2015), but these are domesticated species supposedly adapted to living alongside humans. The fact that so many people attempt to own wolves and wolf-dog hybrids is an interesting phenomena and may speak to many things, including a lack of understanding of what "wild" actually entails, a psychological need to possess something wild, or perhaps even being part of a buy and throw-away society.

Hope (1994) agrees that one reason there are so many people interested in owning wolves or wolf hybrids may be the appeal of owning something wild despite the fact that pet wolf hybrids can be dangerous and have been known to kill children (in the United States at least nine between 1986 and 1994). ${ }^{4}$ He supports this theory by citing ads for wolf-dogs such as, "Own a piece of the wilds! Three-week-old, 98 percent wolf pups for sale!" (Hope, 1994, para 18). Case (2008) also discusses how the phenomenon of wolf and wolf hybrid ownership may be related to a human desire to connect with something wild in an increasingly urbanized world. Case writes:

\footnotetext{
${ }^{4}$ Interestingly, in contrast, in North America McNay (2002) reported no human fatalities caused by wild wolves between 1900 and 2000 and there are only two known cases in the twenty first century (Butler, Dale, Beckmen \& Farley, 2011; Jobin, 2007).
} 
[T]his new fascination with wolves seems to represent an opportunity to reconnect with nature. Viewing wolves as noble and courageous martyrs of the lost wild is part of this veneration. Its most detrimental manifestation is observed in the desire to possess this wildness through ownership of a wolf or wolf-hybrid. (p. 3249)

\section{How Best To Educate}

While my participants agreed that wolf conservation was the primary aim of wolf education and that discouraging wolf pet-keeping was also very important, when asked how best these goals could be achieved, there was less consensus. Indeed, their ideas were quite diverse and at times contradictory. The most common ideas or categories under the sub-theme, "how best to educate," included: the role of dialogue and listening; busting myths about wolves; sharing the "real" wolf; drawing connections between wolves and dogs and wolves and people; creating meaningful experiences and using emotional hooks; the importance of reaching young people; and educating people about coexistence strategies.

I turn first, however, in this "how best to educate" section to a discussion of the tension many of the participants felt between advocacy and education, which for some of them resulted in a desire to "stick to the facts." As noted in the literature review, wolves have been a contentious and polarizing issue for people (Musiani, Boitani, \& Paquet, 2009; Musiani \& Paquet, 2004). I explored this polarizing controversy with the educators and I will outline their ideas on this tension in more detail later. However, given the politically charged issue of wolf survival seemed to influence the educators' stated practices, particularly around the role of science and advocacy in wolf education, I begin here. 


\section{Advocacy Versus Sharing Science}

Seven educators felt that their job was to share only scientific information about wolves (e.g., physiology, ecological benefits, social behaviours), leaving visitors to make their own informed decisions whether or not to be "for" wolves and wolf conservation.

Five other educators, however, shared how they encourage participants to take action by, for example, making petitions available at the centres/programs or giving visitors ideas about pro-wolf actions they might take. Finally, for five of the educators, it was not clear during the interviews that they saw a tension between sharing science and encouraging advocacy.

I found it interesting that all seven educators who stressed the point that education was about sharing the science and ecology of wolves rather than taking an advocacy position worked at centres or programs in areas where wild wolves reside and thus where controversy is more heightened. Monique stated her philosophy of sharing science succinctly:

There's a facet of people who are pro-wolf, who want the centre to take a stand on issues such as hunting, as using dogs with wolf hunts, trapping season, you know... all the issues that face wolves. But that's not been our philosophy since we started. Our philosophy is to try to present all the information that is available, the current science as we know it, and allow people to make up their own decisions.

Likewise, Gwen explained the philosophy underlying how she educates:

...meeting the visitor where they're at, giving them scientific information in a way that they can understand it, and then encouraging them to form their own viewpoints from that information.... I mean our goal as a facility is to be very neutral in the way that we present our information. Of course we're for animal conservation, but we don't take a position on how that should be done.

Five of the seven described how this philosophy played out for them when conversing with visitors. For example, Matthew explained, "One thing we're real careful about here 
is just never to tell people they're wrong, never to say, 'Hey, you're wrong.' Never to use the word 'but'... Use phrases like, 'This is what we understand from the science, this is what the data tells us.' Approach things in a very neutral manner."

In contrast, five educators stated that rather than strictly sharing the science of wolves, encouraging advocacy was part of their role as wolf educators. Spencer described how after coming to their programs, visitors are sometimes motivated to act on behalf of wolves. He said, "It could be a simple email to say, 'I support wolf recovery'... or it could be getting them to come back here, supporting wolves financially.” Ashley also described how wolf education can involve action for wolves; she said:

I want them to care. Take away a call to action. When they leave here I want them to think wolves are important, and here's one thing I can do to help... I make sure that when we leave programs, we always leave them with a take-away. For little kids, that can be something as simple as, 'Hey, maybe you should talk to your friends and let them know that wolves aren't scary.' Just something simple.

Similarly, Shelley was clear that she hoped people leaving her programs were taking action, including political action. She explained that part of her messaging includes the lack of protection and the inhumane management of wolves by governments in Canada (federal and provincial). For example, she informs people how wolves are still poisoned in Alberta, trapped in British Columbia and Alberta, hunted from helicopters, or sometimes captured then sterilized and released. She stated:

For me, I like the fact that I've changed a point of view. I like it when people say to me, "Wow, I didn't know that! That really goes on?" And I'm like, "Yeah." And then they're mad. Like when you tell them stats and information about the government or something stupid, they're, then they look like they're on the hunt, right? They look like they're going to do something. And I hope most people do. 


\section{Advocacy Versus Sharing Science: Discussion Points}

The tension around the role of advocacy in wolf education and the potential influence of location or place is an important discussion point here. Researchers have found that both human attitudes towards wolves and human-wolf relations vary by country or region (Bath, 2009; Chapron et al., 2014; Kellert et al., 1996; Williams et al., 2002). According to Gruenwald (2008), the interplay between environment, culture, and education is messy due to "the uniqueness and diversity of cultural and ecological interactions as they are produced and experienced in particular places" (p. 321). Education at these sites, I propose, is indeed influenced by the diverse cultural and ecological relations people have with the land, other animals including wolves and livestock, and each other. And while this study is not extensive enough to make a definitive conclusion that the educational philosophies and pedagogical approaches of the educators here were influenced by the existence of wolves in the local region and the level of controversy surrounding wolves in each region, I suspect that local context does indeed play an important role. I do not think it is a coincidence, for instance, that the "share only scientific facts" philosophy was expressed exclusively by educators who worked in regions where wolves roam and where the controversy is more intense. Still, it is also important to note the most vocal participant for including advocacy in wolf education also worked at a centre in wolf territory. Mind you, the presence of wolves and the controversy obviously could be highly motivating for an advocacy position too. Nonetheless, this exception leads me to propose that while place or regional context did influence the educational philosophies and practice of my participants, it was only one 
contributing factor in an amalgam of various contexts that include the physical, social, cultural, political, and familial.

Those educators who stressed presenting only scientific information argued that visitors should be free to come to their own conclusions about wolves based on their personal beliefs and values. I found this to be a common theme in research and writing about wolf education as well, with many arguing that information about wolves and human-wolf conflict should be provided but education should not be one-sided or provide visitors with “answers” (Fritts et al., 2003; Switalski et al., 2002; Troxell et al., 2009). What goes unacknowledged in such an approach, however, is that science or knowledge gained through science is never objective or value free as others writing about wolves have made clear (Bekoff, 2014; Jickling \& Paquet, 2005). Further, encouraging action does not necessarily equate to indoctrination, although I recognize that there may be a fine line here, as some researchers have explored (see: Jickling, 2005b; Taylor, 2009). In the recent International Handbook on Environmental Education Research, the editors state in their broad definition of environmental education that it includes "developing the agency of learners in participating and taking action" (Stevenson et al., 2013, p. 2). The philosophy of encouraging advocacy in wolf education also aligns well with humane education. For example, one goal of humane education is providing students with accurate information about the situation of other animals (Weil, 2004), which is represented well by Shelley's practice of elucidating for people the manner in which wild wolves are being killed by the government. Similarly, the goal of empowering students by presenting them with a variety of possible choices they could make that benefit the 
earth and all its animal inhabitants (Weil, 2004) correlates with Ashley offering visitors ideas about even small actions they might take on behalf of wolves.

\section{Meet People Where They Are At: Dialogue and Listening}

Whether their goals included encouraging advocacy or sticking to science, 16 of the 17 educators discussed the importance of starting wherever people were at. They described a number of ways in which they did that, including translating the science and ecology about wolves in a way that participants could understand, allowing those with negative feelings about wolves to vent, and structuring programs for particular audiences (e.g., a curriculum appropriate lesson for a group of sixth graders, a wine and cheese event for adults, a full moon event with campfire, music, and wolf howling for families).

For instance, Leyton explained how he bases his presentations on audience interest: "I will always shift my program to answer the questions. If I have to go politically, we will talk about that. If they talk about the environment, then we move that way." Similarly, Ashley said, "I think depending on your audience, the way you teach things is going to change drastically."

Most of the educators seemed to share Jeremy's position: "as you know in the education field, some people's attitudes will never be changed because they don't want them to change." Likewise, Erik said, "education for some people isn't going to do a whole lot, because at the end of the day, they're going to believe what they want to believe." Rather than seeking outright change, these educators felt their job was instead to listen to people and dialogue with them, and through this process perhaps open them up to new information or an alternate point of view or perspective about wolves. For example, Gwen shared: 
[S]o I really like talking to people and I like getting inside peoples' heads and seeing what informs their opinions, and if there's a way that I can help to open them up to a broader perspective, that's always a really fun experience. Whether it's somebody who doesn't like wolves, or whether it's somebody who really likes wolves and can't understand why somebody would ever want to kill them.

Monique, a wolf educator of 28 years, talked about her early days when visitors would come to the centre seemingly just to vent, "Yeah, they've had dogs killed, and somebody needs to know about it.” For her, being a wolf educator is not about contradicting participants. She said, "I remember the days when, you know, people would just come in and just yell and want to argue about something. And our job in education, again, is not arguing. Our job is listening." She went on to explain that this willingness to listen and allow people to vent sometimes opened these visitors to listening to facts about wolves. She continued, "I feel, the more you have an open door policy, and the less judgmental you are, and the more you have an ability to just listen, the more willing people are to be rational." Gwen shared a similar viewpoint: "You definitely... in this role as an educator, get people who just, they want to talk, they want to vent, they want to tell about their experiences, sometimes they're looking for maybe someone to blame, someone to argue with." She continued that even if she personally disagreed, she thought it was important, "as a professional, and as an educator, trying to continue to have a conversation with that person."

\section{Meeting People Where They Are At: Discussion Points}

Whether through experience or knowledge of education, as demonstrated by their ideas and statements outlined and described above, it seemed that most of these educators seemed to be well aware that increasing knowledge does not necessarily result in attitude and behaviour change, reminiscent of Kollmuss and Agyeman's (2002) important 
reminder to "mind the gap." For most of the educators, wolf education needed to be flexible, adapted to participant interest, and in some cases involved listening and dialogue.

Similar to Taylor and Caldarelli's (2004) conclusion with the park educators they studied, my participants seemed to have a mix of ideas about how best to educate. On the one hand, like Taylor and Caldarelli's participants, they discussed education that was participant-centered and could be adapted based on participant interest. On the other hand, as mentioned earlier, there was much discussion about the importance of sharing information and scientific facts about wolves, which would align more with a transmission approach to educating (Russell, 1997). Ardoin and Heimlich (2013) also found a variety of approaches to education when they surveyed a large number of conservation educators. While some of Ardoin and Heimlich's participants discussed more transmission-based approaches, on the whole, the conservation educators' definitions and understanding of education were more nuanced and complex than they had anticipated. My experience here was similar.

\section{Busting Myths and Overcoming Fear}

Education as a means of dispelling myths about wolves and/or helping participants overcome fear of wolves was a topic that eight of my participant educators discussed. Matthew said, "wolves, of course, have a pretty unsavoury reputation, you know. A lot of people think they're extremely dangerous to human beings." Likewise, Denny stated, "the general public, I think that they still have a lot of that misconception that wolves are vicious, they want to attack you, they want to kill you." 
Christina discussed how this fear of wolves plays out when children come to her centre and are so afraid that they do not even want to view the wolves. Similarly, Monty, who works at a centre in Indiana, described how urban children and youth, in particular, are often shocked that staff will go in the wolf enclosures during a program; he told me, "they are just floored that anyone can actually go in with the wolves." Like many of the educators, he then went on to describe how he perceived that media has, and still does, affect people's perception of how aggressive wolves are towards humans.

Ashley, too, discussed the influence of media and stories on the children she sees. For example, she talked about how a very small snippet from the movie Frozen that shows aggressive wolves is commonly cited by children and, she felt, clearly influences their ideas about the nature of wolves. For her, part of educating, then, is helping to bust the myth that wolves act aggressively towards humans. She explains to the kids that, "That's a fairy tale, that's fiction. What's the difference between fiction and truth? And them just realising that everything they see in the media is not what the truth really is." Likewise, Christina tries to stress with her groups "that myths and fairy tales and Hollywood's version of the wolf is not real." She believes getting past that image of the wolf is important and explained, "Because I think once people are able to get past that, they're a lot more accepting to just learning about the wolf. You know, once they lose that fear of them."

\section{Busting Myths and Overcoming Fear: Discussion Points}

It comes as no surprise to me that these educators discussed the significance of the media since the portrayal of wolves in media and stories is often negative (Houston et al., 2010; Prokop, Usak \& Erdogan, 2011). While I found lots of literature discussing the 
cultural portrayal of wolves, (e.g., Boitani, 1995; Fritts et al., 2003; Midgely, 2001;

Thomas, 2001), there seem to be few studies that explicitly investigate the relationship between how the wolf is portrayed in cultural folklore and stories and people's subsequent attitudes. One exception is Prokop et al. (2011) who, when they investigated children's attitudes towards wolves in Slovakia and Turkey, did find some evidence to suggest that the manner in which wolves are depicted in cultural stories influenced how they were perceived by children. For example, when wolves were portrayed negatively in stories, the children were more likely to have less sympathy for them and greater fear of them.

Within a humane education context, activities have been developed to explore the links between cultural portrayals and subsequent attitudes and treatment of wolves such as Selby's (1995) “Big Bad Wolves” activity in his Earthkind book, or an activity described in Bell and Russell's (1999) chapter in the book Teaching for a Tolerant World. Bell and Russell's activity has students explore words used to describe wild candids (including wolves), investigating how these words are connected both to cultural stories as well as how they impact people's understandings of wolves, coyotes, and foxes.

While the actual danger to humans from wolves is very low (McNay, 2002; Smith, 2007), concerns about human safety still inhibit people from supporting wolf conservation (Smith, 2007). Some of the cultural stories portraying wolves as evil, villains, and/or dangerous have been around for thousands of years, especially in western culture (Fritts et al. 2003). Simply telling participants that these stories and myths are not true may not be enough for them to overcome a fear that is deeply rooted. When I dug deeper during the interviews and asked these educators about how best to bust myths and 
overcome fear, they suggested that experiencing actual wolves made the most difference, which I will describe in chapter six.

\section{Sharing the "Real" Wolf}

The idea of busting myths about wolves often went hand and hand with the idea of educating people by sharing the "real wolf." This concept of the "real wolf" meant different things to the eight educators who mentioned it but was, in part, a response to the extreme perceptions they had encountered, whether it was those who love wolves and romanticize them and put them on a pedestal, or those who loathe them and can see no good reason that wolves should survive and thrive. Jeremy articulated it well:

We don't say things like all wolves are great and good and all the ranchers are terrible and stuff. We really just try to lay it out there what the reality of wolves are, and say they're just another animal in the forest... They have a right to be here just like a chipmunk has a right to be here. But we don't try to say that wolves can do no wrong. I've seen that type of mentality before and I think that's a recipe for disaster..., because eventually wolves are going to do something that humans will disagree with, and when that happens, that whole philosophy comes crashing down.

Along the same lines, four educators stressed how they agree with participants who raise the topic that human-wolf conflict is real, whether that is competition with hunters and trappers for the same prey, occasional predation of livestock, or the danger of wolves to dogs. For instance, Matthew shared, "if somebody comes up and complains about that wolves kill elk, I mean, you agree with them—absolutely, elk are the primary prey, it's the main thing they hunt in the Northern Rockies."

During my program observations at two wolf education sites, I saw evidence of acknowledging with visitors the reality of human-wolf conflict and competition. Both at the Algonquin presentation and the midwestern United States program focused on wolves 
feeding, the educators acknowledged that wolves can compete with humans for prey (e.g., deer), and that wolves sometimes predate on livestock.

When it comes to a predator species like wolves, some people may be uncomfortable with the image of a wolf pack running down and killing a deer, elk, or moose. But many of the educators felt that teaching people about the actual wolf necessarily meant that killing and predation was part of the story. For example, Rick described a diorama at the visitor centre in Algonquin Provincial Park that shows a dead deer on a frozen lake and scavengers feeding on it, and said, "Some people go, 'Well why would you do that? Why would you show nature that way?'... because it's true, and who are you to say that the individual deer is more important than all the scavengers that are surviving on it? So I think it's important to have education, to show people how things really work."

\section{Sharing The "Real" Wolf: Discussion Points}

When Samuelson (2012) conducted a visitor survey and content analysis at one wolf centre in Montana, she concluded that the centre attempted to offer a balanced portrayal of wolves, including depictions of them as both solitary and social as well as representing them as having both negative and positive interactions with humans. This supports my finding here, that the intent of eight of the educators was to share with participants the "real wolf."

\section{Making Connections: Wolves Are Like People, Wolves Are Like Dogs}

Some educators argued that one way to educate visitors was by making connections between wolves and dogs and/or wolves and humans. They felt that by making such connections, visitors might either understand or empathize with wolves 
better. In a later section I discuss how the majority (12) of the educators shared personal insights about the similarities and differences between dogs and wolves and between wolves and humans. Three of these educators explicitly discussed making use of these connections for visitors as an educational strategy. While only mentioned in this way by a few, I nonetheless feel these educators made some valuable points that are worthy of attention.

Monique discussed a program offered at her centre where participants observe, research, and discuss wolf behaviour over a day (including staying overnight at the centre). She described how during this program, she compares the observed wolf behaviours to dog behaviours. She felt that this has been a good strategy as many people live with, and are familiar with, dogs. She explicated, "So next time their dog does it, what do you think they are going to connect it to? They're going to remember, 'I saw a wolf do that!" She clarified her intent in making those connections:

It makes [the wolf] a little less killer, predatory, and a little more social. And that's the piece that I always think everybody, when you ask people what's the first thing when they think about wolves, they always think they're vicious and aggressive. But nobody says, "They're social and family oriented." When you make the connection to dogs, people tend to think, "Well, yeah, that's a social behaviour my dog does"... So I think that's the piece that is really exciting for me, to see that connection [made].

Leyton also reported helping people make connections both between dogs and wolves and wolves and humans. He described a typical presentation where he points out how humans are similar to wolves in that they, too, mark their territory by fencing their yards, numbering their houses, or placing a "keep out" sign on their bedroom door. He asks children:

"Why do you have a fence around your yard? Why? Because you're marking your territory. Why do wolves mark their territory? Because they are telling 
other wolves and other predators this is where we live. This is where our food source is. Do you want somebody coming and breaking into your house, eating out of your refrigerator? No, that's why you mark your territory. You put locks on your doors." So, I try to bring it home and keep it at home and get them to relate to why we mark our territory. We do it, but we do it differently. We're not doing it with urine and scent..., we need to see that we're not all that different.

For Shelley, trying to get participants to see the similarities between humans and wolves is key to helping them empathize with wolves. She told me about a young visitor to the centre who said to her husband Casey (a fellow owner and educator) that his father trapped marmots and considered the wolf to be a pest. In response, Casey asked the youngster whether perhaps his father was a pest in the marmot's eyes, trying to get the student to empathize with what life might be like for the marmot. Shelley explained this teaching philosophy further:

We get people asking us questions that we always reply to as, "Would you like that to happen to you?" and then when they say, "No." I say, "Well, think about [the perspective of] another animal: a wolf, a deer, an elk, whatever, it's still a mammal, it still has feelings, it still raises its children. They're a learning species, they're intelligent, so think about it that way."

She told me that she has found that people often struggle in comparing the experiences of other animals to those of humans, however.

\section{Making Connections: Discussion Points}

All three of these educators were making connections for visitors at least in part to evoke empathy or understanding. For Monique, the goal was comparing wolves to dogs to help people get past the idea of the wolf as vicious. For Leyton and Shelley, it was about stepping into another animal's skin to understand their behaviours and experiences. This is reminiscent of approaches often taken in humane education (Selby, 1995; Weil, 2004). 
Such an approach may be important indeed. As Fox and Bekoff (2009) argue, in the debate around wolves the one perspective that has been missing is that of the wolf. I contend that bringing other animals into the equation, as subjects worthy of their own lives is important, and developing empathy can help with this. Samuels (2007) suggests that part of the process of learning cross-species empathy includes first understanding that other animals have emotions, followed by learning to recognize how other species express these emotions. At least for the three participant educators above, helping people understand and recognize that wolves have emotionally and socially rich lives was important.

Many humane and environmental educators have discussed how developing empathy and/or care for other animals necessitates educational approaches that are grounded in not just scientific facts, but also emotions and direct experiences (Bell \& Russell, 1999; Bell, Russell \& Plotkin, 1998; Fawcett, 2002; Jickling, 2005b; MacPherson, 2011; Watson, 2006), both of which I discuss in the section that follows. For example, MacPherson argues that forming connections with companion animals, and through these connections being able to imagine the experience of the other animal and developing empathy, could be useful in educational contexts. She discusses how these experiences can lead students to "a deeper understanding of their personal, felt identifications and relations with the greater-than-human world" (p. 89).

Discussing wolf-dog comparisons as the educators in this study did may have potential pitfalls as well. Case (2008) worries that comparing wolves and dogs might lead to some negative consequences in how both wolves and dogs are treated. For one, Case explains how when people perceive the dog to be a civilized version of the wolf, 
"bad" dog behaviours are blamed on its shared genetics with wolves which can deepen negative perceptions of wolves. As well, Case (2008) believes popular dominance and hierarchical training strategies for dogs that rely on comparisons to their wolf cousins are often based on inaccurate or romanticized ideas about the social structure of wolf packs; for example, contrary to some public perceptions, packs are in fact dominated by "cooperation and social group cohesiveness" (pp. 3249-3250) more so than dominance and aggression.

\section{Meaningful Experiences and Emotional Hooks}

Closely related to making connections and developing empathy, seven educators discussed the importance of creating meaningful experiences, or ones that appeal to people's emotions, as a key element of their approach to wolf education. Four of these seven lamented that most children today have fewer experiences in nature, but that they felt that such experiences were vital if people are going to understand and care for the environment as well as other species. Noel asserted that because experiences in nature are less common for children these days, experiential environmental education can be even more impactful. He said, "[S]howing [children] some of the simplest things that, you know, I learned as a child in the outdoor world, it's great seeing their minds broaden, their eyes light up... Where they'll definitely remember. It's like experiences [in nature] are even more novel to them now."

Returning to the role of science, some educators stated that in order to make an impression on participants, more than sharing facts about wolves was needed. For instance, Spencer said, "You know, science is only science. People don't believe it. I mean until you experience something, sometimes you just don't believe it." He argued 
that wolf recovery would not happen through telling people facts, but rather would require getting them to care. He stated: "And if we can get other people to open up their heart, then wolf recovery would happen."

The most well attended wolf experience I have heard about is an interpretative program at Algonquin Provincial Park where staff take an average of around 1,600 people during a single program to hear wild wolves howl (see Figure 1). Rick, who leads the program, discussed how research in environmental education shows that an increase in knowledge does not mean people will change their behaviours to be more environmentally friendly. He felt that part of the reason why the wolf howl program has been so popular is the emotional connections made and the experience itself. $\mathrm{He}$ explained:

Our program isn't about telling people everything about wolves, but what if we could make an emotional connection with them? What if we could get them to care because they had this incredible emotional experience? Like hearing wild wolves howling? Because if you can have that hook, then maybe for the rest of their life, they're going to go, "You know what? I'm interested in this animal."

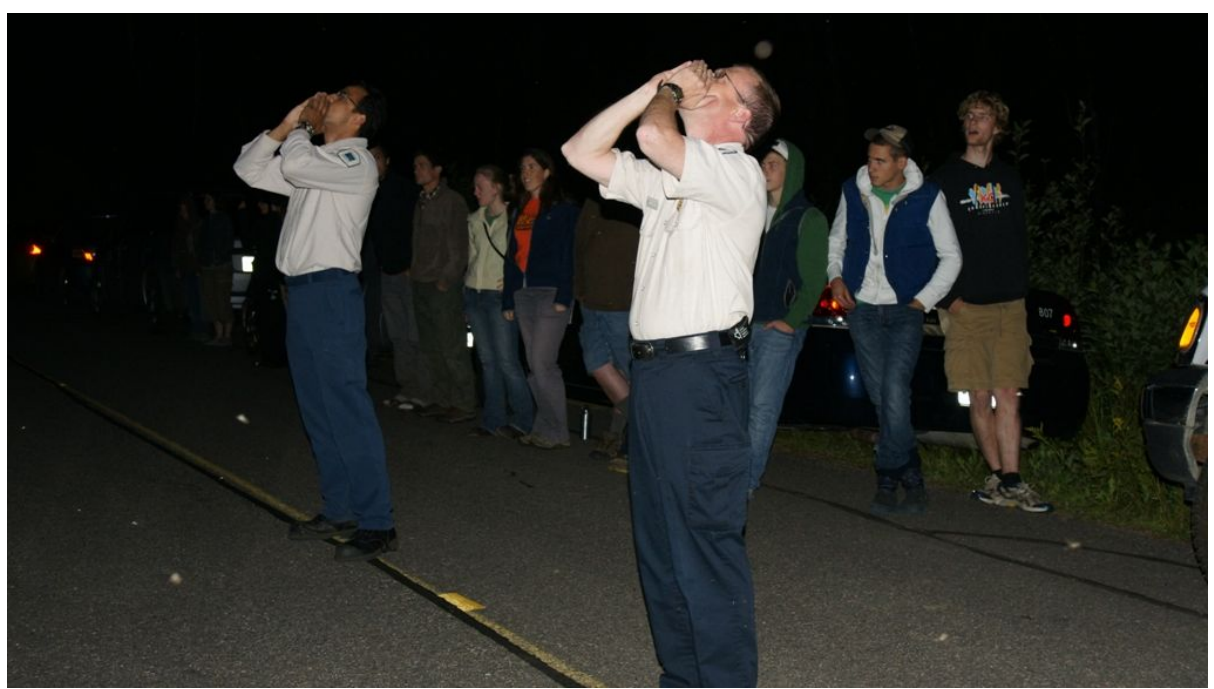

Figure 1. Algonquin Provincial Park Wolf Howl. C Algonquin Provincial Park 


\section{Meaningful Experiences and Emotional Hooks: Discussion Points}

Appealing to emotions and creating meaningful experiences are not new approaches in environmental education. Creating connections to, and encouraging care

for, nature and other species through meaningful experiences is what Sauvé (2005) called a "naturalist" approach to environmental education. According to her, this approach has a long history and often involves both cognitive and affective "encounters in/with nature via experiential approaches" (p. 14). Indeed, much of experiential outdoor education is based on this very premise.

Some research supports taking this approach. For example, discussing the power of experience-based approaches after having examined the experiences of teachers and students at 12 environmental education centres in Australia, Ballantyne and Packer (2009) concluded: "The most engaging, effective, and enduring learning experiences in the context of learning in natural environments occur through experience-based rather than teacher-directed strategies" (p. 259). Likewise, Liddicoat and Krasny (2013) conducted a review of environmental education research and found that childhood experiences outdoors are an important common factor influencing adult environmental attitudes and behaviours. Similarly, in another review, Dillon et al. (2006) examined research on outdoor experiential education spanning ten years and concluded that especially when "carefully planned, thoughtfully implemented and followed up back at school" (p. 110) these types of learning experiences can be very beneficial and effective. Specifically related to wolf education and outreach, Willard (2008) interviewed 20 educators and like the educators in my study, her participants stressed the importance of 
offering people new experiences as one of the three main strategies they employed to help the public understand the complexity of wolf controversy.

However, despite the fact that outdoor and environmental experiential education is often based on the idea that these types of experiences lead to greater care for animals, the environment, and/or environmental issues, some researchers argue that it is often taken up in simplistic ways (Kollmuss \& Agyeman, 2002; Russell, 1999). The phenomenon is far more complex than is often portrayed in linear descriptions of nature experience automatically leading to knowledge automatically leading caring and then to action (Russell, 1999). Russell (1999) worries that while "much of environmental education theory and practice rests on that very assumption" (p. 127), that it is important to define and problematize both the meaning of "nature" and "experience." These can both be seen as socially constructed terms with their meanings influenced by history and culture as well as open to individual interpretation. She encourages educators to consider the relationship between experience and story (or metanarratives), stating: "The stories we tell ourselves about nature and human/nature relationships influence what, if any, nature experiences we seek out, and then, our interpretations of such experiences" (p. 127). Similarly, Kollmuss and Agyeman (2002), in looking at the relationship between environmental knowledge and awareness and pro-environmental behaviour, also suggest that it is by no means a linear progression, but rather is made up of a mix of "knowledge, values, and attitudes, together with emotional involvement" (p. 256) along with a number of other internal and external factors such as personality and culture. I am not suggesting here that the wolf educators were incorrect in their assertions about the effectiveness of meaningful experiences and appealing to emotion, just that there are unexamined 
assumptions made about experiential education and its outcomes that are worth considering.

Turning to the topic of emotions in environmental education specifically, MacPherson (2011) investigated what role emotions and experiences with individual companion animals may play in developing a sense of care and concern for the environment and other species. Despite the differing context (pets versus wolves), her argument about the educational potential of developing emotional attachments to individual animals has some relevance to what my participants discussed above. MacPherson argues that without an experiential-based emotional connection as a foundation, it may be difficult for people to extend their care to larger environmental issues. She writes: "This capacity to recognize and respond to suffering and death depends on experience-such capacities are unlikely to be cultivated in the abstract" (p. 89).

Some researchers argue that rational and/or cognitive ways of knowing are often privileged in education and advocate for the importance of making room for the knowledge and learning that comes through emotion and/or care (Jickling, 2005b; MacPherson, 2011; Russell \& Bell, 1996). Jickling (2005b) writes about "the epistemological importance, or the 'knowing' dimensions, of feelings, care, and love" (p. 104) and how when there is discussion of "values, ethics, and politics" (p. 104), it is impossible to claim objectivity because these values arise out of what we care about. The educators above obviously identified the importance of emotional connections and ways of knowing as well. I return to this topic again in chapter six, when I discuss the emotional and experiential aspects of seeing wolves. 


\section{Kids are Key}

As implied in statements made about visitors that contemporary children have limited contact with nature and wolf educators' work can help address that, thirteen of the participants explicitly discussed the importance of working with children. Six stressed how reaching and educating children is a big part of changing attitudes towards wolves in the long term. Denny said, "We're really trying to get the younger generation. I think we have to start there first and get them...growing up, not to have this fear of wolves and [that] wolves are needed in nature for a reason." Erik described how attitudes towards wolves are often tied to culture and he felt that children and young people are more open to hearing factual information about wolves and learning about the "real" wolf because they may be less aware of the controversy or not yet fully marinated in the particular cultural values and beliefs. He explained:

I mean the big thing is educating the kids. So a lot of the people who are antiwolf right now are, it's again, it's really cultural and being that it's so emotional with a lot of people, it really kind of prevents them from listening to the facts and figures. And you know, you can shove science down some of these people's throats all day, but at the end of the day, they're not going to listen to any of it... Teaching kids to look at wolves as wolves, and not as these angels or demons, I think that education's huge. And, really, that what's going to change over time, the attitudes of people, is through education.

\section{Kids are Key: Discussion Points}

Certainly there is some evidence that participation in environmental education programs has an effect on the attitudes, knowledge, and behaviours of participants (whether children or adults) when it comes to the environment, wildlife, and/or conservation, both in the short and long-term (e.g., Bogner, 1998; Caro, et al., 2003; Dettmann-Easler \& Pease, 1999; De White and Jacobson, 1994; Glick \& Samarapungavan, 2008; Liddicoat \& Krasny, 2013; Visscher et al. 2009). However, as 
discussed in the literature review there are many factors that influence the outcomes such as the length of the program, the manner in which programs are presented, and the effect of supplementary and supporting educational efforts to name a few. I found only one study looked specifically at wolf education for children. This study of fourth graders focused on whether pre- and post-visit activities to a wolf centre enhanced science learning in a treatment versus control group (Glick \& Samarapungavan, 2008). While both groups had improved science knowledge about wolves post-trip, the group that experienced supplementary classroom activities before and after the trip learned more. There was little indication in the article what the children specifically learned, so this study is only of minimal relevance as the educators in my study were discussing the importance of changing children's perceptions and attitudes about wolves more so than their science knowledge.

One thing that did not come up in the interviews was the ethical dimensions of environmental education with children, but is worth pondering. For one, children can be aware of and sensitive to the size and complexity of environmental concerns and catastrophes, and it is important for educators to focus on nourishing positive environmental experiences, critical and creative thinking, and encouraging hopeful and solution-oriented participation while consciously avoiding instilling or perpetuating mentalities of doom and gloom and despair (Kelsey \& Armstrong, 2012). Further, while my participants may be correct in suggesting that fostering, or changing, attitudes in children may be easier than with adults, as noted earlier there is a fine line between education and indoctrination (Taylor, 2009). In Jickling's (2005b) discussion of navigating this line between advocacy, indoctrination, and education, he uses his own 
passion for wolf conservation in the Yukon as an example. He discusses the dangers of coercion or indoctrination when working with impressionable students, but also suggests that, despite the pitfalls and messiness, that environmental educators nonetheless need to help students examine and disrupt taken-for-granted and often invisible cultural assumptions about the environment and other animals and encourage them to explore their values and beliefs so that they can become active citizens advocating for the issues they care about.

Similarly, in a chapter looking at wolf education, Taylor (2009) specifically stresses the importance of avoiding indoctrination: "educators do need to be aware of the potential dangers for indoctrinating their own values and beliefs into others, especially children" (p. 165). Taylor discusses the importance of respecting each learner's autonomy and that education should allow learners to come to their own conclusions and value judgments. This clearly resonates with much writing in critical pedagogy as well (e.g., Breunig, 2005; Darder, Baltodano \& Torres, 2009; Giroux \& Giroux, 2006). For example, Breunig (2005) shares how implementing aspects of critical pedagogy into formal environments necessitates student-centred learning which includes "valuing student voice, promoting and practicing dialogue, shared decision-making, and valuing their previous experiences and their ways of knowing" (p. 117).

\section{Coexistence Strategies}

For seven educators, one of their goals was helping people live and coexist with wolves on the landscape. Michelle said, "So that's usually my biggest thing, is just that we have all these other animals that we have to learn to live with them, and not just against them." Some centres, for example, were involved in educating about and/or 
funding non-lethal livestock anti-depredation techniques. For example, Christina discussed how the California Wolf Center promotes coexistence by forming relationships with those who might come into conflict to wolves. She said:

So a lot of what we do is actually going to these places, and talking to ranchers and hunters and these stakeholders and trying to connect with them, form a bond, and really show respect for them, and work with them to do what's best for the wolf. We have a lot of experience in the southwest doing that: in New Mexico and Arizona. We actually fund non-lethal techniques. So things that ranchers can do to prevent wolves from interacting with their livestock.

Similarly, Monique and Noel talked about different co-existence programs at their centre (they both worked at the same centre), such as a wolf "helpline" that people could call to get help with a "problem" wolf. Monique explained, "We'll either come out and assess it, we'll do some techniques to get that wolf out of your yard. We'll give you some pointers. If your neighbours are feeding wolves, we'll come and have a chat with your neighbours."

Four educators talked about how teaching participants about other species such as deer, elk, and moose can also help promote co-existence. If hunters understood some of the other factors related to deer and moose mortality such as high snow years and disease, for instance, they might be more tolerant to wolves. Monique stated, "So I hear more people now, that are hunters, who used to blame it all on wolves, think about the winter effects on white tailed deer, who didn't prior to education."

\section{Coexistence Strategies: Discussion Points}

While these educational efforts have much potential for mitigating human/wolf conflict, it is also important to remember that the majority of people who visit wolf centres likely already have positive attitudes towards wolves (Black \& Rutberg, 2007). Therefore other ways of reaching those who hold negative feelings and beliefs about 
wolves and/or those who frequently come into conflict with them are needed. Reaching out to the wider public through coexistence programs such as the ones discussed here by the educators above has seen some success in Europe (Chapron et al., 2014). Coexistence strategies may be especially important in areas where wolves have been extirpated for some time because when wolves disappeared from these regions, so too did the techniques for coexisting alongside them (Chapron et al, 2014). Still, there is likely a limit to what education about coexistence techniques can do. For instance, if people who come into conflict with wolves see no value in their existence or lack incentive to make changes in their husbandry practices, there is little reason to expect conflict and negative attitudes to decrease (Bath, 2009; Eisenberg, 2014; Fox \& Bekoff, 2009). Nonetheless, this discussion of coexistence strategies shows that some wolf centres are making attempts to go beyond preaching to the converted by working with, and educating not only people who visit their centres to view wolves.

\section{The Challenges and Rewards of Being a Wolf Educator}

I asked all of the educators about the challenges and rewards of their job or volunteer position. For some, the challenges and rewards did not necessarily relate directly to educating. I often heard, for example, that the best part of the job was working and interacting with the wolves, the variety of tasks inherent in the position, or the working environment (e.g., a beautiful place, great co-workers). However, some of the educators did discuss challenges and rewards specifically pertaining to their educational role. 


\section{Challenges}

Most often, the challenges and frustrations I heard about were related to program participants, including dealing with visitors unwilling to consider new ideas, who arrogantly felt that they themselves had more expertise, or those who were apathetic. The most common frustration, expressed by seven educators, was some participants' inability or unwillingness to be open to new information or seeing another perspective. Jennifer summed it up:

I think it's probably the public. The people who come in and have a preconception and nothing that you tell them can change their minds. They have their minds made up that wolves are bad or all wolves are gods and it just doesn't matter what kind of education you try and give them, that's what they're going to leave with as well.

Gwen found when she first started educating the public about wolves, she had to accept that this sometimes meant visitors were not open to hearing different viewpoints, especially given the wolf issue was often such an emotionally heightened one. She stated:

Wolves definitely hit more of an emotional nerve with people... And so, what was challenging for me at first, and still sometimes can be challenging... is accepting people's opinions and beliefs and values for what they are and not trying to change them in any way. Really practicing listening to people.

It was not just those visitors who disliked wolves or had misconceptions about wolves that were a challenge for the educators. Four educators discussed how pro-wolf visitors could be as challenging as anti-wolf people to educate. Noel said, "I've had a lot of situations where, you know, a lot of folks come here with a pro-wolf attitude and they want to speak more than they want to listen." Similarly, Erik said, "You know, you always hear stuff from the anti-wolf crowd, but the pro-wolf crowd can be just as bad with misinformation." He went on to describe his frustration with how some people 
anthropomorphize the wolf. He compared his experience of visitors' perceptions about wolves with their beliefs about bears, which he felt they generally understood to be wild animals. In comparison, some felt wolves are "these perfect animals... they look at wolves and they tend to connect with them more, and they kind of look at wolves through human eyes more, and anthropomorphize more." When it came to dealing with pro-wolf people, Monique described how she had experienced outright hostility from individuals involved in wolf advocacy when she tried to share alternate perspectives. "I've had many, many situations where they're like calling me a murderer or calling me various things... and it wasn't that I wasn't agreeing with them, I was giving them another perspective. They didn't want to hear it."

Along the same lines, Erik, who worked at a centre that rescued wolves and wolf hybrids, expressed how challenging it was to deal with visitors who felt they themselves were the experts. He explained how participants sometimes felt the need to share their expertise and personal experiences, including about an animal they had owned and believed was part wolf: “They’ve convinced themselves it was a wolf-dog and so therefore they're a wolf-dog expert... and they want to tell us all about their experiences and how they handled their wolf-dog, and what they did. And they want to tell the other visitors, so that becomes kind of an issue."

On the other end of the spectrum, six educators discussed how it was challenging when participants were apathetic or just wanted to be entertained. Monty described this challenge well:

Boy, the most challenging is probably overcoming apathy... You can see people losing interest and sometimes people even walk off when you're talking about wolf recovery and all the issues. "I don't want to hear that." You know, they want to be entertained. So you kind of have to mix the two carefully, kind of mix 
things in. So it's not really a huge concern, but it is a challenge..., keeping people engaged, trying to get them to learn without getting them too overwhelmed, I guess, is a way to put it.

Besides these more commonly cited challenges, I also heard about other concerns such as keeping up with bookwork and dealing with the financial aspects of running a program, the impact on the centre when schools, for budgetary reasons, decreased field trips, and frustrations when talking with people who complained that the wolves on site lived in pens or enclosures.

\section{Rewards}

When it came to describing the rewards of being a wolf educator, for eight participants, it came down to sharing. They talked about how great it was to share their passion for wolves, share in an experience involving wolves, or share their knowledge with participants. For example, Noel described his favourite part about being a wolf educator:

If I can observe with visitors [wolf] behaviour and interactions and have that teaching moment where I can talk to visitors and show them, "Hey, that's what that behaviour means. That's what they do out in the wild"... And engage in those conversations, that's probably the best aspect of the job is to be able to engage with folks like that.

Likewise, when asked what she liked best about educating, Michelle responded, "I like giving the tours. I never thought I would enjoy public speaking as much as I do... and it's just what I love to do, it's just something that comes naturally... Like I said, my tours tend to run an hour and a half [laughs]; longer than they're supposed to, because I get so into it."

In a similar vein, some educators really liked seeing participants' reactions when they learned something and/or saw something new and interesting, whether it was their 
reaction to the wolves on site or their understanding of some concept or idea. For example, Jennifer said, "It never gets old to watch people see [wolves] for the first time. I always check them in, and give them the layout of the centre, and I always follow them up, to see what their reaction's going to be." Similarly, Ashley said, "I think my favourite thing is when you see a light bulb click." She went on to describe a grandmother who booked a private tour with her granddaughter who was terrified of wolves and was having wolf nightmares. She described how rewarding it was when, by the end of the tour, the little girl was no longer afraid of wolves.

\section{Challenges and Rewards: Discussion Points}

It seems that the variety of rewards and challenges described by the educators here are complex and probably dependent on a number of factors. After interviewing people involved in education and outreach related to wolves in the western United States, Willard (2008) came to similar conclusions. All of her participants discussed the challenges of education and outreach given wolves stir up "such intense emotions grounded in a multitude of unyielding opinions" (p. 38). What my participants found challenging and rewarding about their work was likely influenced by the educators' own values and beliefs about wolves, their ideas about the goal(s) of wolf education, the philosophies of the centres/programs where they worked, and the attitudes and motivations of the people that visit (e.g., whether looking to be entertained, to learn, or to challenge). 


\section{Conclusion}

In this chapter, I have focused on the wolf educators' ideas about education, namely their ideas about its purpose, approaches, rewards, and challenges. I chose to title this chapter, "The Complexities of Educating for Change," because it became clear to me that their experiences of educating people about a controversial subject like wolves were complex. Contextual factors definitely added to the complexity. As discussed above, one factor that seemed to influence their experiences was the location of their centre and the level of controversy over wolves in their region. For example, local context seemed to have some impact on whether the educators and/or centres took a stand on advocating for wolves or attempted to share only scientific concepts about wolves. A personal contextual factor that appeared to have an impact on many of the educators was their educational background in science as it seemed to permeate their ideas about the purpose of wolf education, how best to educate, and their ideas about the ideal content of wolf education. That being said, adding to the complexity was that many of them at the same time also discussed how focusing on wolf science alone would not be enough to achieve the goals of wolf education, with many citing the importance of emotions and meaningful experiences.

I sensed that most of the educators cared deeply about wolves and wolf conservation and this came through in many of the quotations I chose to include in this chapter. It was clear that this care and passion for wolves and their conservation meant these educators really wanted to make a difference for wolves, and in our discussions about how best to educate about wolves, these deep feelings sometimes appeared to be in juxtaposition to how they believed "professional" educators should act. For example, this 
complexity was evident when they talked about needing to meet the visitor where they are at or to not try to change visitor attitudes. It also shone though when they discussed the rewards and challenges they experienced; their care and passion for wolves meant that visitors not being open to new perspectives or just apathetic affected them more potently. To conclude, the fact that wolf conservation is a controversial subject in North America makes the experience of being a wolf educator nuanced and complex. My participant educators' ideas about and experiences with educating were influenced by their cultural, regional, and personal contexts. They were also very much influenced by their care for wolves and their conservation, which I explore in more detail in the next two chapters. 


\section{CHAPTER SIX}

\section{UNDERSTANDING AND WORKING WITH WOLVES}

In this chapter, I explore two of the big overarching themes that emerged from discussion during the interviews, "understanding wolves" and "working with wolves". First I outline the participants' experiences with and beliefs about wolves. I begin with considering how educators have come to understand the wolf. I examine both their more abstract ideas as well as those they described as having been developed through observing and/or interacting with wolves. Next, I explore the theme of what it means to work with wolves, attending to both the educational and ethical aspects of doing so. Finally, for those who worked directly with the wolves at their centres, I discuss these human-captive wolf relationships.

\section{Understanding the Wolf}

For all of the participants I interviewed, being a wolf educator meant in part, understanding the wolf. What such understanding entailed varied and including knowing about wolf biology and ecology, contemplating the roots of human-wolf conflict, or coming to know the wolf through observation and interaction.

\section{Biology and Ecology}

All seventeen educators discussed, sometimes at length, the biology and ecology of wolves. Most often they discussed this in relation to the content of what they were sharing with their participants in their educational programs. For example, when it came to wolf physiology, the interviewees described how they shared information about wolves' bite strength, their coats and colouring, their feet, their size, their yellow eyes, 
and their amazing senses. Other topics included mortality rates, litter sizes, habitats, and territories. I also heard lots about wolf packs, such as how small or large packs can be, and how packs are mostly made up of family members (i.e., a mating pair and litters from multiple years). I was told about how wolves sometimes disperse, travelling long distances to find a mate, creating new packs. Educators discussed pack hierarchical structure (i.e., alphas, betas, and omegas) and how this social structure plays out within a pack. I also heard plenty about the types of animals wolves prey on and the intricacies of what and how they hunt, including their success rates.

I discussed wolf ecology in the "Advocacy Versus Sharing Science" section in the previous chapter and I will discuss it again in the next chapter looking at wolf conservation. I do not want to be repetitive so suffice to say that, for these educators, knowing about and sharing the science of wolves was an important aspect of their jobs. Sharing scientific information and research about wolves was stated directly in the missions of many of the centres as I discussed in chapter four. Since at least twelve of the educators had backgrounds in science and/or natural resources, knowing about and sharing information about wolves through a scientific framework appeared, for the most part, comfortable for them. On a few occasions, however, educators discussed experiences with actual wolves that conflicted with their scientific understandings of wolves and I will describe some of these instances as they come up in later sections.

\section{Understanding The Polarity of Human-Wolf Relations}

Moving from the biology of wolves, I turn now to the diverse ways in which humans relate to wolves which was a great source of discussion in the interviews. As noted in the literature review, many human cultures have had tumultuous relations with 
wolves. For example, in the lower 48 states in the United States post-European settlement, wolves were purposefully and almost completely eradicated save a couple of small pockets in northern Minnesota and on Isle Royale in northern Michigan (Boitani, 2003). Twelve of the educators I interviewed worked in places where wolves were either still extinct or had been reintroduced through government programs. Especially in areas of reintroduction, heated debate about wolves persists (Houston, et al., 2010). Gwen works in one of the reintroduction regions and describes the range and polarity of opinions she sees in their visitors: "We have the whole spectrum from people who love wolves and think that they should be pets, and they want to hug them and pet them, to people that absolutely hate wolves and think they should all be destroyed." During the interviews I discussed human attitudes towards wolves with all of the educators and why they think wolves and wolf survival continues to be, at least for some, such a controversial and polarizing issue.

The roots of polarity: Why some people hate them. Ten educators described the extreme negativity towards wolves that they had encountered, whether it was at their centre or program, in the local community, or on the Internet. For example, Erik shared how he had seen wolves described as "these ecosystem-destroying machines that are going to wipe out the elk herds and then hunt people next... The Internet is terrible for that kind of stuff. Like, 'Once the elk are gone, keep your kids inside'.” In discussing some people's negativity towards wolves and/or the divisiveness over wolves, some key common points emerged as important considerations for the educators. While there is some overlap, the two main ideas raised by the educators were: 1) the influence of culture, movies, and fairy tales; and 2) the issue of government power and control. 
Culture, movies, and fairytales. When it came to understanding the negativity expressed about wolves, eleven of the educators discussed the influence of culture, particularly focusing on myths, movies, and fairy tales. As Jennifer explained, "You just have people who don't know any better than the fairytales where it's the Big Bad Wolf and they're feared and they're hated, and [they should] be rid from your town or your range or wherever you are." Similarly, Gwen said, "In North America, that European influence, I think definitely gets very deeply into our psyche, with the Big Bad Wolf and Little Red Riding Hood and the fear of the wild places, and the unknown, and the idea that wild places need to be tamed."

When Christina became an educator, she was surprised to find how much influence fairytales and Hollywood stories had on people's perception of the wolf. She said, "It was an eye-opening experience for me, you know, I couldn't just blow that off as that's just Hollywood, because it has a lot of effect on people, and their perception of wolves." She found that addressing those stories and disrupting ideas about the wolf's aggressiveness and danger to humans thus was an important part of her work as an educator.

Michelle compared the wolf's cultural portrayal with another common predator, the bear. "I like to say on my tours, Little Red Riding Hood versus the Teddy Bears. You always have Big Bad Wolves, bad wolves, bad wolves. And then we've got Smokey the Bear, we've got Cinder the Bear now who was rescued in the most recent fires in California. All these cute little Teddy Bears." She went on to cite statistics that demonstrate how bears are more dangerous to humans than wolves yet people appear to be much more tolerant of bears: 
And I always kind of make fun about it, because we live in Colorado, we have bears everywhere. I'm sure you do in Canada too. And every day on the news, "There's bears in my yard! There's bears, bears." And it's like people love the bears. But, it's, like I tell people on my tour, you can have a bear break into your house, and sit on your couch in the living room, and people will be, "Oh, look at the cute bear in my living room!"... But we blame the wolves, The Big Bad Wolf, because that's what we grow up with. Can you imagine the pure panic that would happen if we had a pack of wolves sitting in your backyard eating your trash instead of a bear?

Two educators delved more deeply into how and why these culturally based stories came about in the first place. Rick believes the roots are ancient:

I think it goes back to a lot of our evolutionary history. You know, I think naturally we humans, as we evolved, we were afraid of predators, we feared predators, we tried to conquer predators, and we wrote stories about them, and we wrote songs about them. And we have fairy tales which all demonize this animal, you know, that's the fear that we have of them.

Matthew, too, contemplated the roots of fairy tales and folklore. He thought that stories emerging from cultures with domesticated livestock may have played at least some part in the wolf's negative portrayal.

Folklore's entire purpose is to reinforce cultural norms, is to instill them at an early age. So reinforcing a cultural norm that wolves are bad and need to be destroyed was happening generation after generation after generation. And so it was brought over from Europe into the Americas, and continued, and continues.

Government power and control. When it came to the origins of attitudinal polarity, four educators also discussed how feelings about wolf reintroduction were sometimes intertwined with the issue of government power and control. For example, when asked what might be driving the controversy around wolves, Spencer said, "I mean a lot of it is the fact that the government put wolves back into places, you know, it's this whole, 'Not in my backyard' mentality. 'How dare you put them here!'” Similarly, Matthew pondered, "I wonder if some of that is part of the problem here [in this region] is that wolves were wiped out, and they were brought back, and it's seen as an overreach 
of the government..., forcing wolves onto people that didn't want them." Likewise, Ashley, whose centre is involved in the Red Wolf Species Survival Plan (SSP), reflected on what might be fuelling people's negativity in the region of reintroduction, North Carolina : "I would say in North Carolina the issue is more government control. Areas where people think you're spending all this money to save these Red wolves, so what about my kids?... Shouldn't that go to education, shouldn't that go to whatever is going to directly help their families instead of this random animal that they feel they've lived fine without?"

Other roots. Other less common ideas about the roots of the polarized views of wolves and hatred for wolves also emerged. Two educators suggested the polarity partly represents an urban/rural divide and three of the interviewees felt that views were sometimes bound up with people's political beliefs and political affiliation. In the United States, for example, Spencer said that support for wolf recovery is "political. And it's more of a red state versus blue state ordeal, I guess. The blue states have been a little more supportive, the democrats and such. And they're making rules that are much more wildlife-friendly." Likewise, Erik discussed the urban/rural and political aspects of the wolf debate in Washington State. Those in the west of the state where the big cities are, he explained, are more environmentally minded and typically support wolf recovery while those on the eastern side where farming and ranching predominate do not want wolves. Ironically, the eastern side is the area the wolves have reinhabited. With regards to those in the east, he said, "One thing they don't like is the people that want wolves

${ }^{5}$ Centres involved in the Red Wolf or Mexican Grey Wolf SSP, like the one where Ashley works, are sometimes located in states far from the release areas. 
back in the state aren't the ones living with wolves. And they're the ones that don't want wolves, and yet they're the ones that have to live with wolves."

A variety of other ideas about what fuels hatred or dislike of wolves was also discussed. Shelley, for instance, explained how humans have tried to eradicate them yet they keep coming back. She said, "I think that's why people are so miffed by them, and hatred, because we're not able to control it." Matthew felt that part of the issue is that humans and wolves are so much alike - two social animals that have competed over prey and livestock for centuries: "And I think because we see a lot of ourselves in wolves, we've had a difficult time getting along with them." Alternatively, Rick explained that our past treatment, or more accurately mistreatment, of wolves may have not always been fuelled by negative attitudes and feelings. He gave the example of how killing wolves used to be considered good science. In fact when Algonquin Park was formed in 1893, killing wolves appeared in the Park Act. At that time, he stated, "In order to protect wildlife, we had to destroy bears, wolves, and other noxious and destructive animals. And that's in the Park Act..., they really thought they were doing the science. If you wanted to have deer and moose and other animals, the only way to ensure that long-term was to destroy the predators." Finally, two educators discussed how bad news gets more press than good. Monty, for example, felt part of what fuels wolves' bad reputation is negativity bias, that is people, including the media, tend to remember and report negative experiences more often than neutral or positive ones.

Why some people love them: What is it about wolves? While on the one hand, educators speculated why some people have strong negative feelings towards wolves, many also explored with me why other people have such strong positive feelings about 
them. What is it about wolves? Certainly, it is clear that there is some kind of draw. As outlined earlier, consider the number of people that are visiting these centres and programs to see wolves - in many cases, tens of thousands annually. And while the educators did describe encounters with visitors who had negative feelings and beliefs about wolves, the majority (13) agreed that most people coming to their wolf centres and/or programs attended because they liked wolves.

During the interviews, several ideas came up as to why some people feel drawn to wolves. Two educators felt it was, in part, a human need or desire to feel connected to nature. For example, Rachel speculated that some people want a wolf as a pet to feel a connection nature: "Because they want to feel that connection to nature, to the woods, to the wild, so they get a wolf as a pet." Similarly, Rick believes that part of the reason so many people have attended the howling program at Algonquin Provincial Park since it was first initiated in 1963 is that the wolf represents a connection to nature. He explained, "I think we have, over time, lost our connection to nature. You know we don't live on farms anymore, we're not intimate with nature. We live a suburban lifestyle now. But I think at the core, we [still] want this connection... I think there is a symbol here, that this is a truly wild animal that we want to connect with." He went on further: "I honestly believe it has to be more than just the wolf. It has something to do with our nature itself, and that this is a connection back to something that's very special to us."

While these educators thought the wolf's draw might be motivated by a desire to connect to nature, four other educators suggested that part of why people might be drawn to wolves especially is because of their similarities to dogs. Humans have a long history of living with dogs and they speculated that this may create a feeling of affinity towards 
wolves. Erik mused, "Part of it, I think, has to do with dogs, that dogs can be similar to wolves... And they think, oh, if I have a great relationship with my dog... But then they make that same connection with wolves, that wolves are these perfect animals, and that sort of thing."

Why, exactly, people are drawn to wolves may be hard to unravel and there surely is no single one reason. Nonetheless, it is clear that there is something powerful at play for some. For example, one educator talked about participants for whom the wolf was a "spirit animal," and other interviewees used words like "mystique," "majestic," "magical," and "regal" when describing the wolf. For instance, Shelley said, "It's this spiritual thing that they have over people," and Denny stated, "I think there's just that mystique when you say 'wolf'." Denny went on to describe a conversation with a participant: "This one guy, he told me, he kind of apologized and says, 'I really take myself as a big, husky, burly guy,' which he was. He said, 'But when you got them wolves to howl', he said, 'I just lost it.' It just does something. I like to say it does something to your soul." Likewise, Rick described a lifelong Park interpreter's reaction to hearing wild wolves howl for the first time: "he wrote [in a blog post] that when those wolves started to howl, it just went right through him, and it will be something he will remember for the rest of his life."

\section{Understanding Polarity: Discussion Points}

Many academic writers and some attitudinal research support these eleven educators who discussed the influence of culture, expressed in various forms, in explaining some of the polarity when it comes to human attitudes towards wolves (Boitani, 1995; Fritts et al. 2003; Nie, 2002; Prokop et al., 2011; Shelley et al., 2014; 
Schlickeisen, 2001; Thomas, 2001). While some of the educators discussed how a culture's stories influence people's attitudes, some also noted how these stories also emerge out of certain relationships and reinforce cultural norms. Like Matthew who suggested folklore reinforces cultural norms, Boitani (1995) proposes that, historically, those cultures that relied predominantly on domesticated livestock, especially nomadic shepherding cultures (e.g., cultures from northern Europe), encountered the most conflict with wolves and also typically had the most negative attitudes towards wolves. These are often in evidence in their cultural stories. I wonder, then, whether cultural stories might eventually change when people learn to coexist better with wolves and/or whether changing stories about wolves (e.g., having them appear as the "good guys" in movies) could influence cultural attitudes and beliefs?

While explicitly named by only four of the educators, the discussion of government power over how land is used influencing human/wolf relations is supported by the social science literature looking at human attitudes towards wolf reintroduction. When examining peoples' attitudes about wolves, issues of government/institutional power do indeed often surface as they did in Enck and Brown's (2002) survey of residents in Adirondack State Park, Scarce's (1998) interviews with residents on the border of Yellowstone, Skogen et al's (2008) study of resident narratives in France and Norway, and Skogen's (2001) investigation of young people's attitudes to wolf conflict in Norway. Wolf conservation, and presumably education for wolf conservation, is much more complicated when wolves become bound up with, and sometimes a platform for, larger issues such as government and/or institutional power. 
When it comes to passion for wolves, some researchers and academics point to a change in environmental values over the past 50 years that may be connected to urbanization and how this shift has likely influenced the public's attitudes towards wolves (Boitani, 2003; Fritts et al., 2003; Kellert et al., 1996). As a portion of the educators suggested, some people's positive inclination toward wolves may not have a simple cause, as there are likely many factors involved including emotional and spiritual ones. Kellert et al. (1996) would agree. In a literature review focused on human culture as it relates to large carnivore conservation in North America, they concluded that large carnivore species are important not only for their ecological or economic significance, but also for "the many emotional, intellectual, and even spiritual benefits provided by these charismatic mega-vertebrates" (p. 988). Likewise, in discussing the importance of wolves in Ontario in a Backgrounder document, the Ontario Ministry of Natural Resources (2005) points out not only the ecological, but also the social, cultural, economic, and symbolic importance of wolves for people. This discussion about people's various positive reactions to wolves and wolf encounters offers a nice segue to the next section.

\section{Understanding the Wolf Through Experience}

All of the educators I interviewed reported learning about wolves through observing and/or interacting with them at their respective centres and programs and consequently, "Understanding the Wolf Through Experience," became a sub-theme. In fact, ideas about wolves gained through observation and experience was the topic that generated the largest amount of interview data. In the section that follows, I outline some of the key concepts discussed, including wolves' personalities, the social nature of 
wolves, wolf communication, wolves' abilities and senses, how wolves are similar and different from dogs, and how wolves are like humans.

Wolves are individuals with personalities. When talking about the wolves who lived at their centres, 14 of the educators specifically described different wolf personalities, whether discussing wolves' preferences for certain people, the intricacies of wolf-wolf relations (i.e., who got along with whom and why), describing favourite wolves, or telling stories about unique characters. For example, Denny talked about two wolves, "two brothers, Lucas and Lincoln, they were born in the same litter, same time, same age and they look similar, but they are totally different personalities, absolutely the opposite." He went on to describe how one of the brothers is timid, the other much more outgoing. Likewise, Shelley talked about the personalities of the wolves at her centre:

You've got so many personalities, right? You've got the bully, you've got the passive, you've got the mom type. Like you've got Flora, she's a little know-itall... And then her brother is like this laidback, whatever. But he's a chicken too, right? So he doesn't want to try anything new. And then Wiley's the bully and then Moab's just this noble type of wolf that everybody admires, and he's just got this chill personality. So they are all their own individuals.

Two of the educators explained how, when it comes to wild animals, people often think of them at the level of species, but working with an animal like the wolf in a captive setting helped them see how each wolf was unique. Christina, for instance, said, "You know a lot of people, when dealing with wild animals in particular, for some reason, kind of see them all as the same. But working with the wolves that we have here, it's so easy to see that every single one is very different from each other." Further, Monique explained that dealing with wolf personalities was part of what made her job interesting. She said: 
Every wolf is different, every animal is different. Every day is different. The social dynamics of every individual is different. And that's the piece you don't see in wildlife biology. And you don't see that at the population level. You see that at the individual level. And that's the best part of my job, and that is that part that connects people.

Given that so many of the educators (12) had science and natural resource backgrounds, I suspect that for many of them, the experience of getting to know and understand individual wolves was an interesting contrast to their understanding of animals garnered through their formal education. Monique stated this outright: "I think the best part is to see everybody's own personality. As wildlife biologists we're taught to think about the population, not the individual. We're taught that from day one, biology 101."

The social nature of wolves. All 17 educators discussed things related to the social nature of wolves (see Figures 2 and 3 below). While a few only touched on it briefly, some discussed what they had observed about wolf social interactions and family structure at length. Having observed wolves, most believed that the social dynamics of wolves are quite complex. For example, Jeremy said, "I think once I got my hands on them, or least my eyes on them, I think that their society and their communication among each other is actually more complex than any book could ever describe it as."

When it came to social behaviour, 12 educators discussed how wolf packs were families, often comparing and contrasting them to human families. For example, Michelle stated, "a wolf pack is a family. So it's basically a mom and dad and all their kids." Within the theme of family, the educators told me about wolves who had adopted puppies that were not their own, how packs would care for, nurture, and teach young wolves, and how wolves would play together. Shelley said: 
I guess another stupid question we get too is, "Do they play? Do they care for each other?" Of course they do, they're families, they're pack animals... One of the other amazing things is that they are so loyal to each other... Maya has raised every single one of our puppies here, she never birthed, and she has helped take care of each one of them.

Likewise, Rachel explained how they would put puppies with their "ambassador pack" on site as these pack members were well socialized with humans: "We will put [them] in with the ambassadors because ... the adults teach the puppies how to behave around us, and that it's okay to meet new people."

Educators also explained how, within a pack, the inter-wolf dynamics and relations were unique. For example, they reported that wolves enjoyed or preferred the company of certain other wolves. Christina said, "So you do see stronger bonds between individuals within a pack." Similarly, Jennifer talked about the pack at the centre where she worked, describing how "the girls will all kind of hang out together and they all snuggle. They will never go snuggle with him [another wolf]. They just seem to have that sister relationship, you know?"

Educators also told stories of how wolves helped each other out, whether it was a low-ranked wolf in the pack who had food given to them by another wolf or an older or injured wolf who was shown care or given protection. Christina, for one, told me about a wolf in their Rocky Mountain Grey wolf pack who had some mobility issues and could not get to the food quickly:

So one of the younger wolves would actually bring him food before he ate himself. So he would grab food and bring it to him, and then he would go back and eat. So it's things like that where you're like, wow. That's supposedly a wild animal, and even so, they can show these caring and loving behaviours that a lot of times I think humans forget to do. 
Along the same lines, Jeremy said, "I've seen evidence of kin selection with wolves as well, which is yet to be proven." He offered the example of wolf siblings Wahotts and Weyekin to illustrate his point. Wahotts was a low-ranked wolf or the male omega of the pack at the time.

He would risk himself to steal the food — and he would grab some food, usually get disciplined for it [by a higher-ranked wolf], but still make off with the food. And he would literally carry it to the other end of the enclosure and drop it in front of her [Weyekin, his sister] and whine, turn around, and go back to the food. So he was literally providing her food and things like that. I'm like, how is that benefitting him?

I also heard stories about how wolves would show anxiety and mourn when separated from their pack for even a short period of time and how wolves who had escaped the enclosure would not run away, but would hang around to try to get back to their pack in the enclosure.

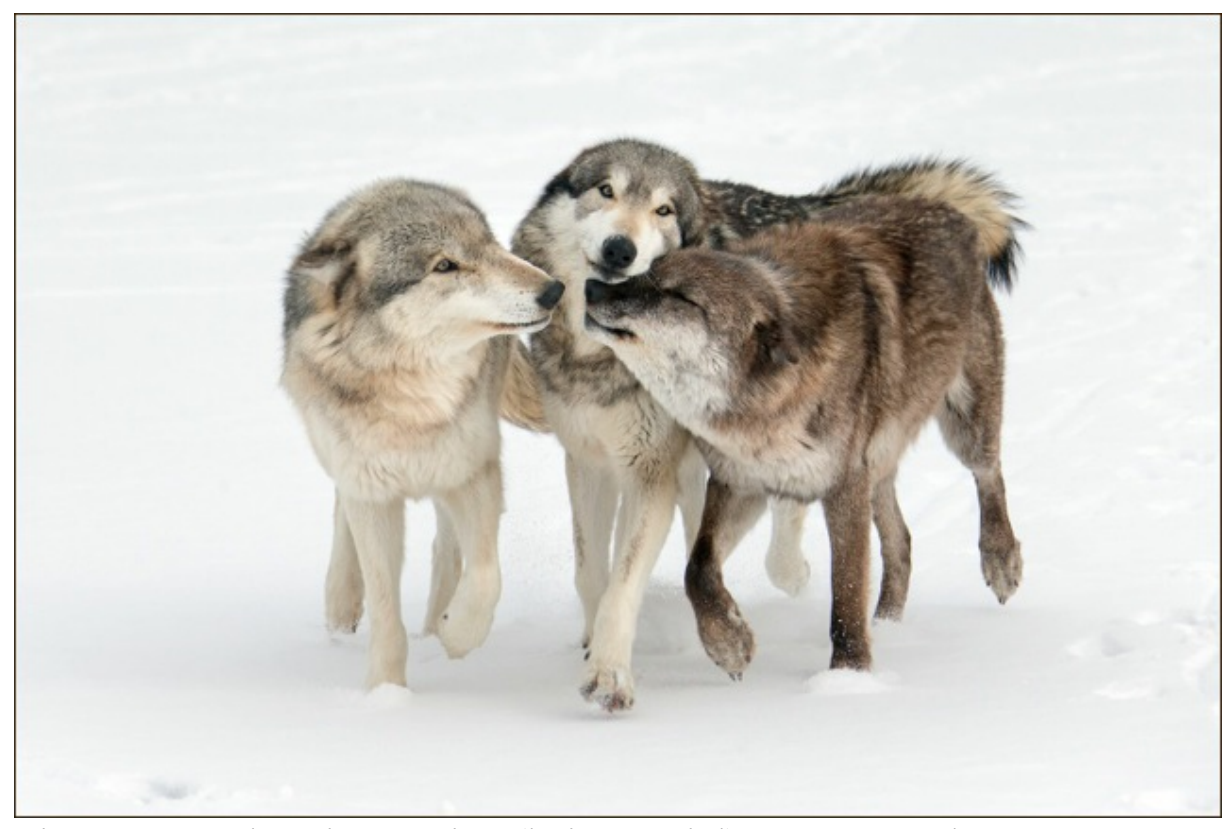

Figure 2. Wolves interacting (being social). (C) Monty Sloan 


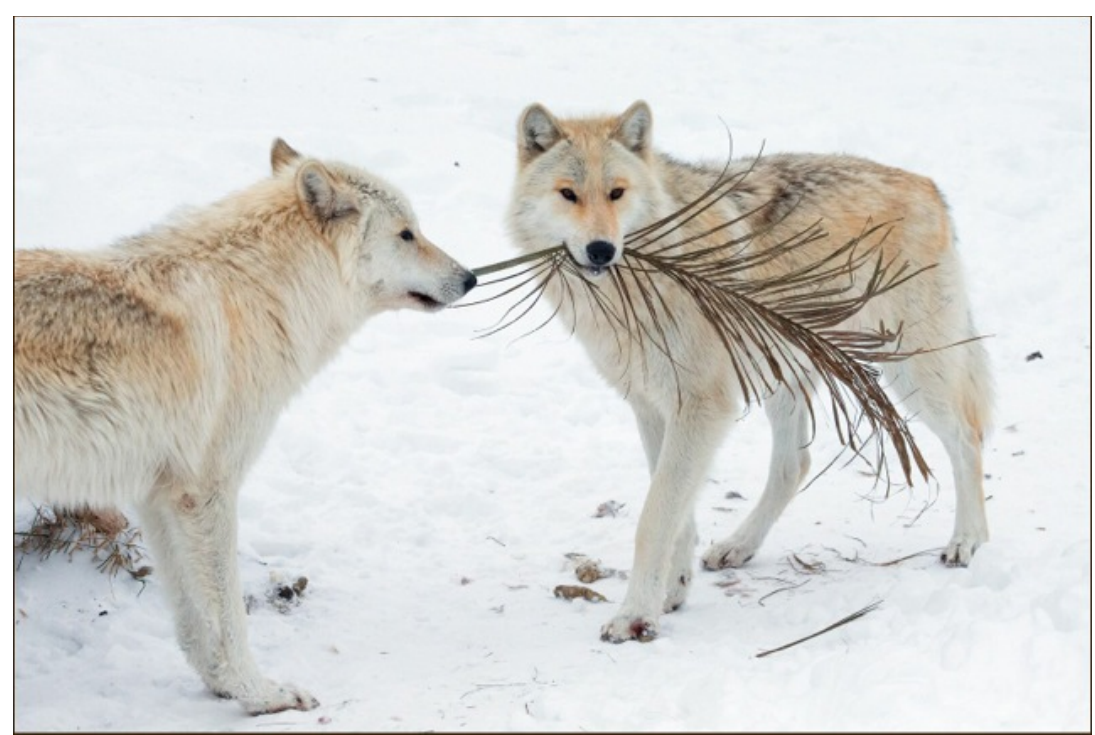

Figure 3. Wolves interacting (being social). CMonty Sloan

Wolf communication. One of the social behaviours educators observed and wanted to discuss was wolf communication. It was discussed frequently enough (by 13 of the participants) that I decided it deserved its own small section. The educators discussed how wolves communicate using body language, vocalizations, and scent (e.g., communicating territory to other wolves by scent marking).

Body language. Nine educators discussed the body language of wolves. For instance, Christina said,

It's usually all body language that we see. So tail position is really important, you know, whether or not they have their hackles raised or not. They'll do a lot of standing over one another, and the one laying down is the more submissive one, and usually the submissive wolves do a lot of muzzle licking of the more dominant animals as well.

Four educators offered me photos that show wolves interacting and communicating, a few of which I include below. In these photos, body language, facial expressions, and submissive and dominance behaviours are portrayed (see Figures 4, 5, and 6). 


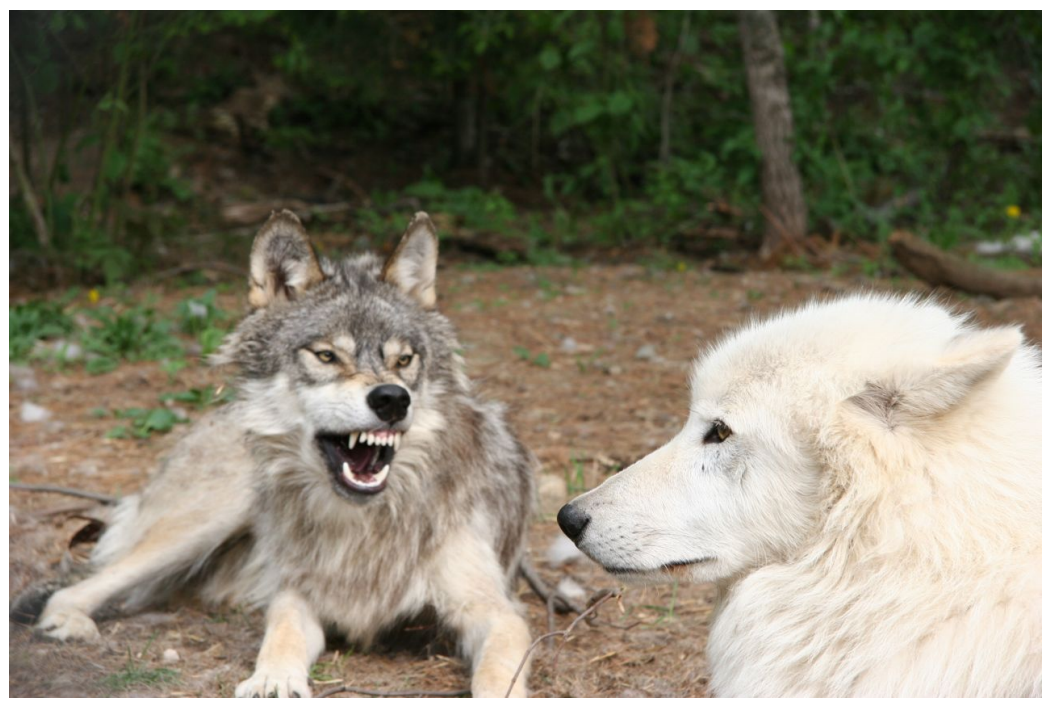

Figure 4. Inter-wolf communication. (C) Wolf Care Staff

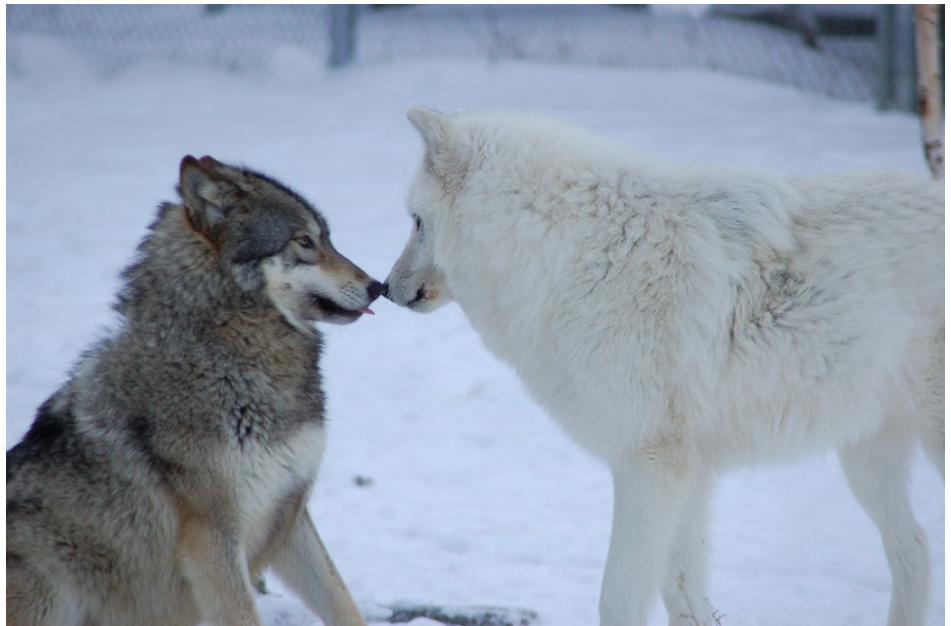

Figure 5. Inter-wolf communication. (C) Wolf Care Staff

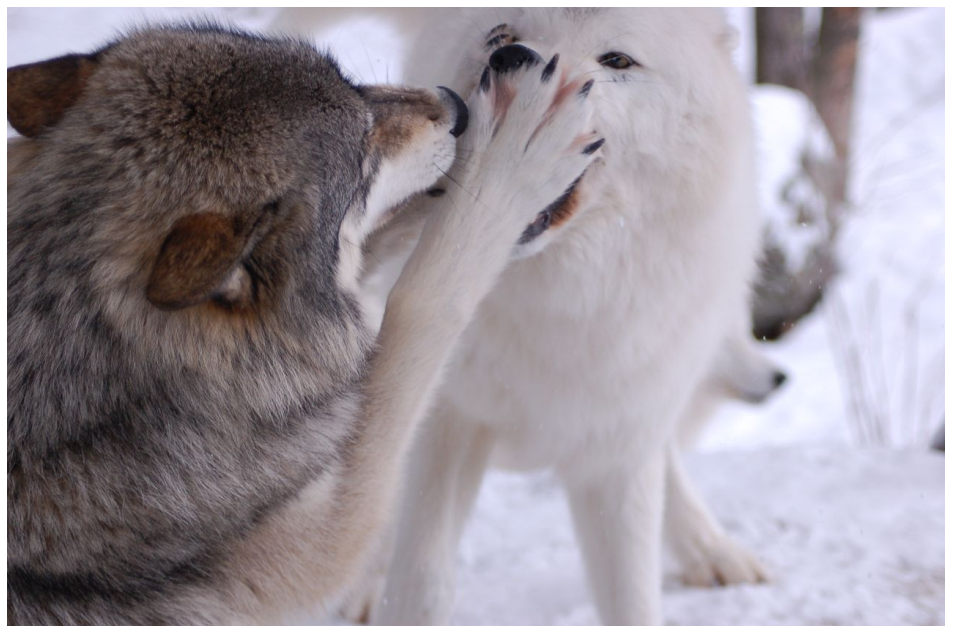

Figure 6. Inter-wolf communication. CWolf Care Staff 
Three of the educators also discussed body language and communication that, at least as yet, may not be completely understood or even decipherable to humans. For example, Denny described the eating hierarchy with the wolves at his site, and how even without signs that were clear to him, the submissive wolves know when they can eat. Jeremy too suggested that there is communication going on that is hard to figure out:

I mean there is some very non-verbal, non-posturing communication that they perform that just boggles the brain. Some of us are even, like, we're not serious about this, but we're like, 'Do they have ESP?' How do they know what each of them is thinking without displaying anything? So it's very, very complex and that's fascinating.

Vocalizations. Leyton was one of 12 educators who talked about wolf vocalizations, especially howls (see Figure 7). He stated, "We know that wolves, they huff, they puff, they whine, they snort, they growl, they howl." While these educators were not sure the wolves were necessarily always communicating something specific when howling, three educators noted that the wolves often howled at dawn and dusk, although they could not say exactly why. I also heard about how some of the educators had come to understand the meaning of certain types of howls and vocalizations. For example, Michelle talked about what happens when certain wolves who are able to walk on a leash get an outing:

I think it's really funny when you get one of the wolves out for a walk. Even if you walk outside the door with a leash, everybody starts howling. And it's a completely different sound from other times of the day, because you can tell that there's just more energy in it. And they're like, "Who's going for a walk? And why am I not going?"

Similarly, Rachel described the commotion when a wolf escapes its enclosure: "I like to say wolves are the biggest group of gossips that you'll ever meet. So if one animal does 
get out, everyone starts talking about it, and being here for a while, you tend to learn what those howls sound like."

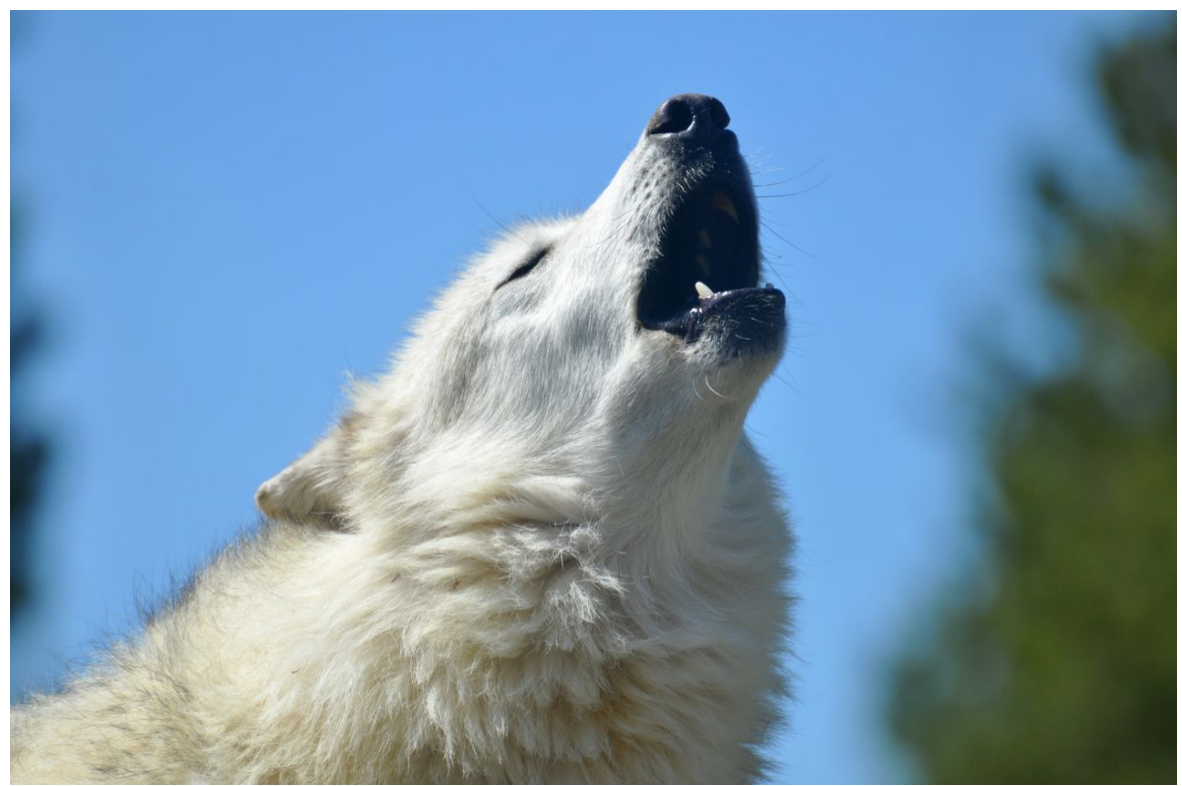

Figure 7. Howling wolf. (C) Courtesy of participant

Mourning howls. Five of the educators specifically described a mourning or separation howl. Christina shared, "When Aspen ended up passing away, Rio howled for so long. And it's just because they have such close ties to one another. And it's just a very different howl than we normally hear. They usually howl sunrise and sunset... But the mourning howls are just very different." Likewise, Ashley explained how before working with wolves, she did not realise how they mourn. She shared a story about one wolf:

Picaron is one of our wolves that was on that site, and he was with his mate, Tanamara for a long time. When she wasn't in the enclosure anymore, he howled for so long and so hard that he permanently damaged his vocal chords and he couldn't howl anymore. So just knowing that they have that feeling, like if we lost a spouse or a mate, that we would feel that loss forever and they...[can feel] the same way. (see Figure 8) 


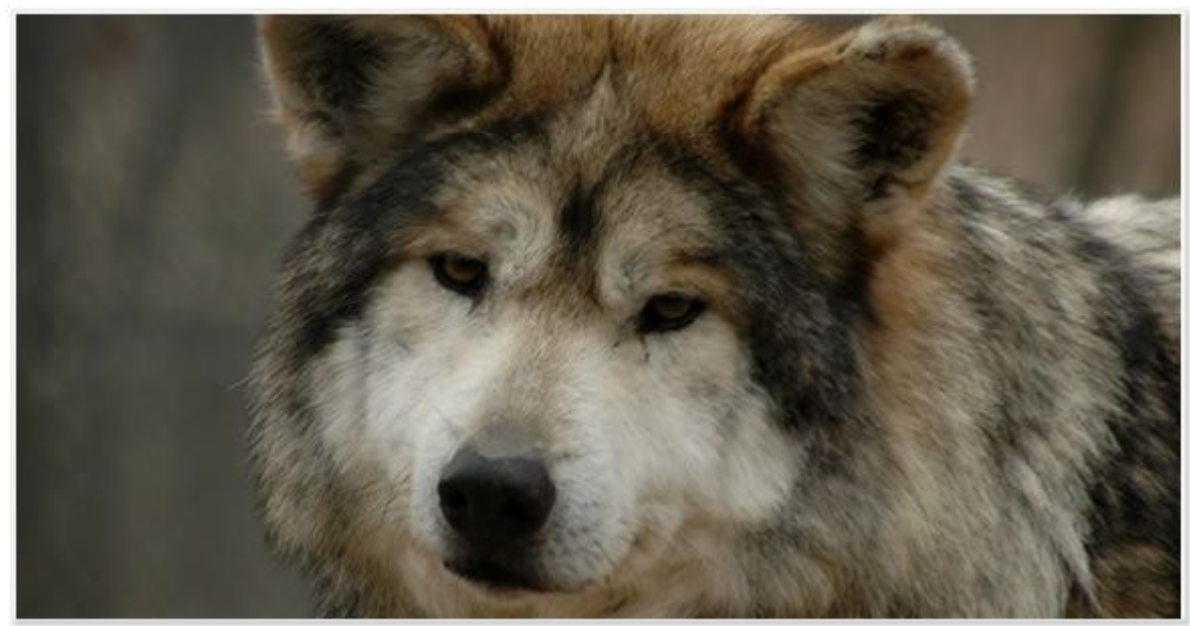

Figure 8. Picaron. (C) Endangered Wolf Center

Wolf abilities and senses. During the interviews, I was told about a range of wolf abilities and senses. Participants explained that wolves are: intelligent, opportunistic, ingenious, loyal, playful, curious, goofy, nurturing, resilient, have amazing strength, saliva with a healing quality, and amazing senses (e.g., sense storms coming, sense human sickness, sense/hear/smell a car two or three minutes away). Sometimes these qualities were discussed in the context of a story. For example, Christina shared a story about Kiska, an older wolf at her site who had been put on a low protein diet for health reasons:

So we weren't able to feed her as much meat as she would have liked. So we were feeding her mainly a kibble that was low in protein. And she didn't like that at all, so what she would do was actually knock her kibble bowl over, so that the ravens would come to eat the kibble. And then she would catch the ravens and eat the ravens (laughs). So they're just so intelligent...

Similarly, Monique shared a story she heard from a beaver trapper that showed the intelligence and ingenuity of wolves: "And it got to the point where [the wolves] just learned to follow [the trapper's] sets [traps] around, you know, so that was their hunting technique, and that's pretty ingenious." 
Four of the educators described how wolves' senses are quite acute. For example, Denny talked about how when someone from the centre went to pick up a roadkill deer, the wolves sensed something and perked up when the vehicle was still two or three minutes away. Similarly, I heard from two educators that somehow their wolves could sense a storm coming. Jeremy detailed, "I can tell you that they can forecast a thunderstorm coming. Yeah, there are indicative behaviours when it's thunderstorm season. They get rowdy, they vocalize a lot, they get super energized. And it's a bright sunny day, and then literally within a couple of hours, we've had a big thunder storm."

Wolves are curious. The most commonly cited quality when it came to wolves beyond their sociality was their curiosity. Eight educators specifically used the word "curious" in relation to wolves. I heard about how wolves were curious about visitors, children, and new or different things (e.g., a stroller, an umbrella, a wheelchair). I also heard how their curious nature could get them into trouble in the wild when humans perceive it as aggressive or react fearfully. Shelley stated:

Like they're so curious too, right? Like, it's unbelievable how curious they are. They're not aggressive and I get those stories all the time [from visitors], "They kind of just snuck out and they were looking at us." Well, if I saw this weird person with a big belly standing there, I'd probably sneak out and see what the heck you were as well. You know, you're foreign, like, "What are you?"

Wolves are similar and different to dogs. Twelve of the educators discussed what they had learned about the differences and similarities between dogs and wolves through their observations and/or interactions with wolves. Since dogs and wolves are both canines and close genetic relatives (Thalmann et al., 2013), it is not surprising that the educators compared the two. 
Some of these educators primarily focused on the similarities. Erik was one of three educators who explained to me that wolves, wolf-dog hybrids, and dogs are sometimes similar enough that the general public cannot tell the difference between them. He said:

So even in captivity, in zoo situations, a lot of times the wolves may have wolfdog in them. And then the vast, vast majority of people that contact us about wolf-dogs, [the animals] are just northern breed crosses [dogs], they're malamutes that have a colouring that looks kind of wolf-y. You know, it's just off the wall. And then people will say, "Oh the dog howls, and it chases small animals, so it must have wolf in it." So behaviour, like bad behaviour a lot of the times, gets blamed on, "Oh, it must be part wolf."

Spencer too discussed wolf and dog similarities, but in the context of how the travelling ambassador wolf Atka at the centre was raised and socialized with a German shepherd. Spencer talked about the things they do to enrich Atka's life since he does not live with other wolves, such as going on outings to the beach and offering variations in diet and toys. He stated, "You know, he's a dog, he likes toys." Gwen also discussed dog-wolf similarities. She described how she used what she learned observing the wolves at her centre to help her understand and work with her own dog who sometimes shows aggression towards strange men. She explained, "One of the things that wolves do when they're showing dominance is, they'll kind of put their paws on top of the back of another wolf. Or stand over another wolf." She went on to explain how she used the same posture with her dog, and "any time he would get to trying to show too much dominance, that's what I would do..., which was really interesting to me that that worked, but it did."

Alternatively, five educators explained how their experiences helped them better understand the differences between dogs and wolves. Michelle said, "I guess the main thing is I didn't realise how different [wolves'] behaviours really are from dogs. Wolves 
or wolf-dogs, they're just very different." She went on to describe how when wolves are puppies, they are a lot more like dogs, but as they mature, they seem to have a much more independent nature than dogs. Monty, too, discussed the wilful nature and independence of wolves, explaining:

The wolf is actually very, very trainable and they will pick up the routine faster than a lot of dogs. The problem is that they don't pay as good attention, they startle very easily, they distract very easily, and when I lecture about wolves, I like to tell the audience that you can train a wolf to do anything that it wants to do.

In fact, Monty discussed the differences between wolves and dogs at great length. He had been involved in comparative research at the centre where he worked and reported that comparing wolves and dogs was one of his key interests. Like Michelle, he explained how wolves mature differently than dogs, which is why he felt socializing wolves at a very young age was important. (Intriguingly, all of the educators who worked at centres with socialized wolves discussed the necessity of socializing them very young.) In comparison to dogs he stated, "With a wolf..., by 3 weeks of age, there's so much fear that the animal will never trust you and will never really form social bonds." He also felt that wolf personalities were more varied than those of dogs because:

$[\mathrm{O}]$ ne of the things that we did in the last few hundred years in creating dog breeds is tried to fix behaviour. And so you have a general expectation of how a German shepherd would work or a leonberger or a lab or any dog... Whereas wolves are all over the place, and even littermates, one will be completely different than the other in terms of its personality.

Amidst discussions of how different wolves and dogs were, two of the educators reported that they had changed their opinions about owning pet wolves or wolf hybrids based on their experiences. For example, while Rachel had previously thought she might like a wolf hybrid as a pet, her experience working with them and seeing how many end up as rescues shifted her perspective: 
So, you know, hearing all of the stories of these animals and just kind of hearing how they came to us, and also doing my research for my thesis on wolf behaviour, helped to open my eyes, and be like, "Oh my gosh, these animals are not at all the same to a dog." You know, they're really difficult to take care of, and that just really opened my eyes.

Wolves are like people. Ten of the educators talked about how wolves are similar to humans. Most often, they compared the social structure of the two species. For example, Leyton stated, "A pack is a family unit with their leaders as the only breeders... They are affectionate and ... [wolves and humans] have a comparable social structure." I heard from three of the educators who felt that, in some ways, wolves were better than humans when it came to their ability to care for each other and understand their role and place in the social scheme of things. For instance, Spencer said:

You know, the social structure, we try not to throw our human emotions onto these animals, but if there's going to be an animal to do so, then the wolf is a pretty good one, because they do have a lot of the same practices and stuff with their family units. You know, they all do their part... Well, wolves and humans, I mean wolves might do a better job sometimes.

Other. Interactions and observations of wolves led to a handful of other unique things that the educators reported learning about wolves. For instance, two of the educators discussed how having observed wolves firsthand helped them understand better the "real" wolf, both the good and the bad. Both Noel and Gwen explained how, prior to working with them, they had overly positive impressions of wolves. Noel said, "When I first had wolves on my radar, they were very glorified to me. They seemed like this allperfect creature that was saving the ecosystems." Both felt they now had more realistic ideas about wolves. Gwen explained, “Kind of what I've learned through watching the wolves and observing them is wolves are an animal, like any other animal. And there are things about wolves and wolf social structure that are really cool and really neat. And 
there's things that are really rough and sometimes hard to watch." In a different vein, two educators who worked at centres with wolves not socialized to humans explained that, through experience, they had learned how very afraid of humans wolves were. Ashley, for one, stated, "You know, I always heard that they were afraid of people and I didn't really know what that meant until I started working here."

\section{Understanding the Wolf Through Experience: Discussion points}

These various learnings about wolves gained through experience with actual wolves lead to some interesting discussion points. First, the ethical implications of understanding other social animals as subjects with unique personalities, a variety of abilities, and/or the capacity for complex social communication are discussed by some environmental and humane educators (Kuhl, 2011a; MacPherson, 2011; Oakley et al., 2010; Pendersen, 2011), critical geographers (Emel, Wilbert, \& Wolch, 2002), and environmental ethicists (Fox \& Bekoff, 2009; Lynn, 2007). For example, in a discussion paper looking at issues of human-animal relations and animal advocacy within the field of environmental education, Oakley et al. (2010) write about the significance of understanding and relating to other animals as subjects: "For some of us, past experiences with nonhuman animals have compelled us to find ways to recognize and honour their subjectivity and our interrelationships with them" (p. 96). One of that paper's authors, Cutter-Mackenzie described her past experiences living and working with farm animals and how that led her to vegetarianism and a concern about the treatment of animals in the meat industry.

Bekoff and Pierce (2009) contend that recent research showing the complexity and depth of social animals' lives calls for a new set of ethics. Historically, when animals 
were seen as having little capacity for emotionally rich lives, humans generally used them how we pleased (e.g., for meat, for research). Especially given recent research, Bekoff and Pierce submit that this ethic is no longer appropriate. While not all of the educators who described the wolves at their programs as complex social animals or individuals with unique personalities discussed the ethical implications of this realization, some of them did indeed suggest that there were implications arising out of their new understandings, which I will discuss in a later section on the ethics of conservation and management.

Second, recent research in animal ethology and neuroscience supports some of what the educators reported learning about wolves. For example, there is research that substantiates the claim of the educators who told me about the capacity wolves have to care for each other, to communicate, and to mourn. Bekoff and Pierce (2009) reviewed a substantial portion of this research and summarized that "recent research is demonstrating that animals not only act altruistically, but also have the capacity for empathy, forgiveness, trust, reciprocity, and much more" (p. 3). Ten educators asserted that wolves are like people and had little difficulty comparing humans and wolves. In contrast, in western culture there is a history of stressing how humans are different from other animals, reinforcing the human-animal divide (Noske, 1997; Selby, 2000). Yet, being fellow mammals, we humans do share a lot of the same characteristics with other social species such as wolves (Bekoff \& Pierce, 2009). I assert that what the educators I interviewed have learned about wolves through their daily observations and interactions with them has great value. While we cannot know everything that goes on in other animals' minds, Bekoff and Pierce (2009) propose we nonetheless can learn a lot through observation: "Look at their faces, tails, bodies, gaits, and most importantly their eyes. 
What we see on the outside tells us a lot about what's happening inside animals' heads and hearts" (p. 43). This certainly echoes much of what my participants told me.

Despite the fact some scholars caution against cross-species comparisons, often grounded in a critique of anthropomorphism, a growing number of researchers posit that empathizing with other animals need not be undesirable or problematic, but in fact may lead to improved ethical interspecies relations (e.g., Fawcett, 1989; Jickling \& Paquet, 2005; MacPherson, 2011). For instance, Jickling and Paquet (2005) write, "We are constantly imagining the world through the eyes of others - our friends, our family, our lover. This is one way that we come to understand them. It then seems a short empathetic step to imagine the world through the eyes of more-than-humans" (pp. 129$130)$.

\section{Educating With Wolves}

The experience of being an educator involved more than just knowing information about wolves, it also included the opportunity to work with wolves. Whether or not the educators had hands-on interaction with the wolves at their various programs and centres, all of them worked at programs where wolves (wild or captive) were part of the visitor experience, thus "Educating with Wolves" emerged as one of the main themes of the interviews. As for sub-themes, I discuss four in this section. Because of their experiences working at programs that included wolves, all of the educators had ideas about what the benefits were for participants as well as about the ethics of using wild or captive wolves for educational purposes. Some also had ideas about how best to care for 
captive wolves and/or the ethics of wolf-care. Finally, for some of the educators, working with wolves meant forming bonds and connections.

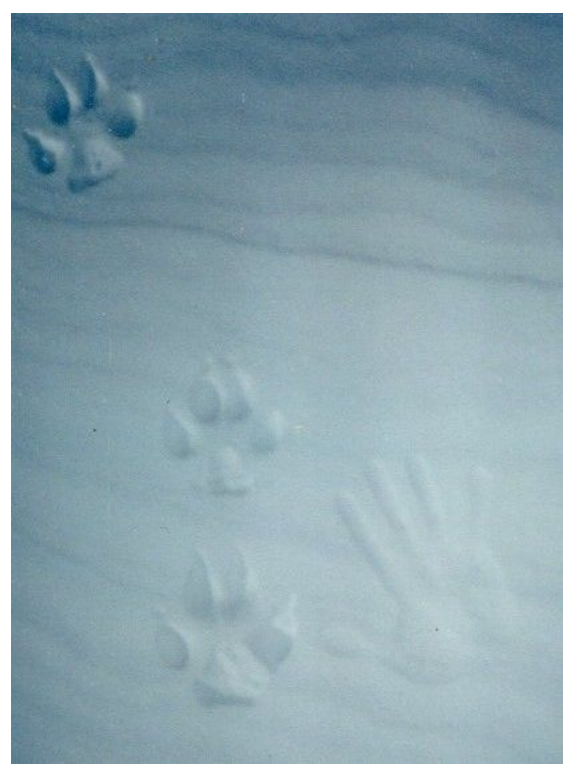

Figure 9. Photograph of wolf paw prints with a human handprint. (C) Wolf care staff

\section{Experiencing Wolves: Perceived Benefits for Participants}

To begin, I discuss the educators' ideas about the benefits of education involving captive or wild wolves. All 17 educators believed that encountering wolves created a more impactful visitor experience than giving a talk or showing a film. The most commonly cited reasons that live wolves enhanced the visitor experience according to the educators included the opportunity for participants to make connections, the visceral nature of the experience, the opportunity to watch wolf social behaviours, witnessing firsthand the wolves' lack of aggression, and using wolf viewing as an incentive to get visitors' attention.

Making connections. The most common reason educators gave as a benefit of wolf education programming with wolves was the possibility of "making connections." Sometimes the idea of connection was vague. For example, I heard plenty of statements 
similar to this one of Gwen's: "It can be hard to make a connection to something you can't see, that's not tangible. And so, having the live animals is something tangible, it's something they can see and something that they can observe, and that definitely helps, and makes our job a lot easier." Other times, the educators were more specific and talked about what, precisely, the connection might be about, such as connecting to something wild, to something living, or to nature in general. For instance, when discussing the impacts on visitors of seeing wolves, Jeremy said:

[I]t's a living being. They can see that the wolf is looking right at them... When a wolf looks back in a person's eyes, I can't tell you how many times people have said, "I feel like they're looking into my soul, they're looking beyond just my eyes,"... So, when you have that kind of interaction happen up close and personal [with] a wolf, I mean I've seen people walk out of our classroom crying because it was so impactful. They're like, "I am so moved, I will never forget this my entire life."

In a similar vein, Monique discussed the impact of human-wolf encounters: "People don't listen. They don't read. I mean, the reality is the way that we get our message across is a wolf staring them in the eye, inches away from their face at the window. And especially kids, little kids, and having that connection to wolves." She went on to explain how the impact for some is powerful enough that having that one experience or connection as a child had led them to return years later and become educators themselves.

Emotions. Eight of the educators discussed how visitors interacting with, or viewing wolves, sometimes evoked an emotional response. Rick believed that this emotional connection is part of why experiencing wolves can be so effective. He said, "I think that's why having a live animal works, because there's an emotional connection." Similarly, Matthew said, "I mean people have an immediate and visceral emotional reaction when they see the wolves." Leyton talked quite a bit about the emotional 
response people had when encountering wolves: "You get a feeling. It's undeniable.

There's no person that I have ever encountered in the crowd that says, 'I don't feel anything.' You feel something, whether it's fear, and whether it's anxiety, whether it's awe, intrigue, admiration, you feel something." Similarly, two educators described how visitors would sometimes cry when hearing wolves howl.

A visceral experience. Tied closely to emotion, another reason eight of the educators felt wolves had such an impact was that the experience was visceral, that it involved the senses. Monty stated, "It's because we're a very visual species. So when you can actually see what's going on in real life and right there, that will grab your attention far better than any movie or Power Point because it's right there happening in front of you. You can see it, you can hear it, you can smell it in some cases." He went on to explain how people need and want those connections to nature and wild animals because we "are so separated from wildlife and nature, more and more so."

While observing a wolf-care session at one midwestern United States wolf centre, I sat on a ledge looking out over a large viewing window into a very naturalized enclosure (i.e., large and wooded, with a pond and a sloping hillside). I was surprised at my own visceral reaction to seeing wolves up close, supporting what the educators discussed. I wrote in my journal:

I see a few wolves and immediately my heart skips a beat. Weird, what is it about wolves? My first impression is that they are dog-like, but at the same time completely different. There is something much more striking about them. Regal? Majestic? Wild? Big? They are definitely large, with long legs. But there's an indescribable presence about them too. Dare I say 'spirit'?

Even through glass, I had a visceral reaction. Nonetheless, two of the educators asserted that nothing could replace the impact of actually touching or having physical 
contact with a wolf. Leyton stated, "It's every educator out there, I'm certain, who has a wolf on a leash, can tell you that that's the difference. When people can see it, feel it, and get hands on it, everything changes. It just changes everything. I wish I could explain it, but I'm not an outsider looking in." Similarly, Rachel said:

I mean you will remember being licked on the face by a wolf for the rest of your life. And that's why we do ambassador tours and take people in with the wolves... We've found that by giving them [visitors] that experience to actually meet the animal, that we're trying to promote conservation for, having that interaction and that intimate experience on a personal level with that animal... gives them a stronger sense of conservation.

Watching wolf behaviours. Seven educators explained how having the wolves interacting with each other and their environment helped people understand wolves and especially wolf behaviours. For example, Ashley said, "You know, me talking in front of a picture and talking to kids is a lot less captivating than me pointing to a behaviour an animal's doing and saying, 'Look at this animal, this is Anna, and this is why she's doing this." Rather than having things like body language and submissive and dominance behaviours described to them, the visitor participants could see it firsthand. Shelley explained, "Especially when they can see them behaving together. They [participants] can see the [social] structure, it makes a big difference." As well, some educators felt that in the presence of wolves, participants were more engaged and curious, asking questions about things they were seeing. Michelle said, "It helps that it's interactive. But when you're actually seeing a live animal and it does something, people can go, 'Hey why are they doing that?' or 'Oh, look at how deep those dens are!'” (See Figure 10).

Wolves are not that bad. Five of the educators discussed how, through seeing captive wolves, participants learned that wolves were "not that bad" (i.e., not as aggressive or dangerous as they are portrayed sometimes in the movies, fairy tales, and 
fables). For example, Jennifer explained that, “they don't look like anything that should be feared. Not that they should be feared, but that's what lots of people think. So it's very cool to watch people experience that." Likewise, Christina felt the biggest thing that

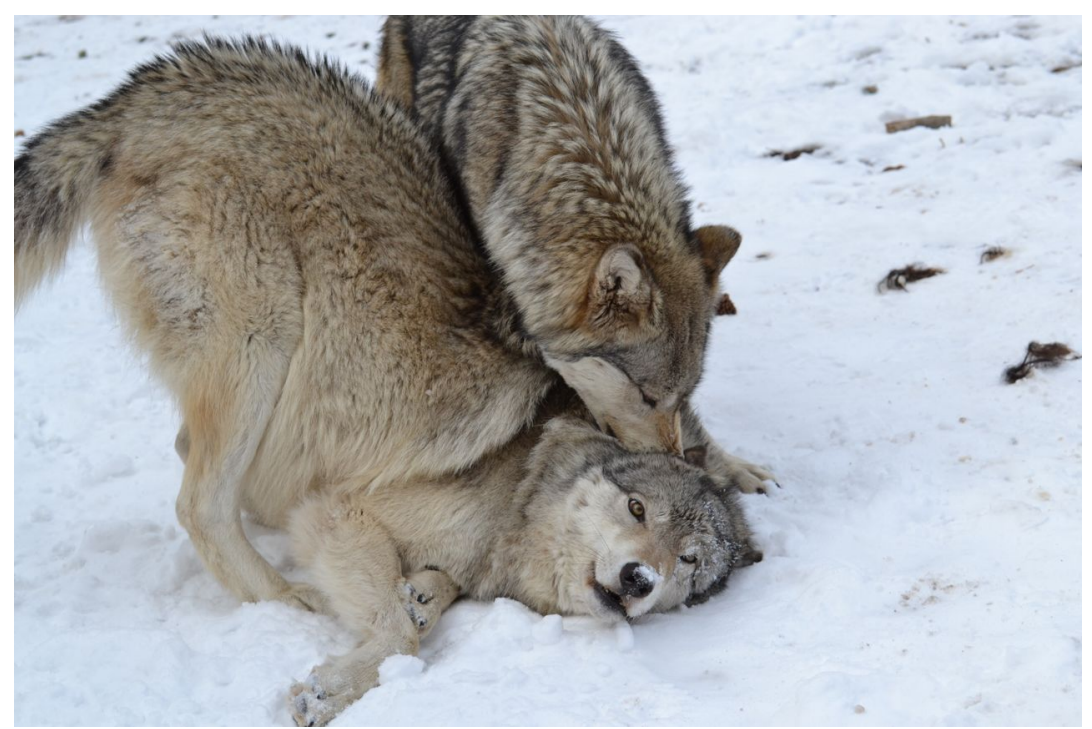

Figure 10. Showing wolf behaviours. CMichelle Smith

helped visitors overcome misconceptions and fears about wolves was seeing them live. She stated, "Our wolves are pretty shy, and they're curious, they want to see what we're doing, but no charging of the fence, no teeth baring or anything like that." She continued, "Seeing them and realising they don't want to attack you. They don't want to eat you. They're not looking at you as a food item." She felt that when participants' witnessed how shy and easily startled the wolves were, they often changed their perception about how dangerous they were.

Wolves as a "carrot." Finally, two educators talked about using the opportunity to see the wolves onsite as an incentive to having participants listen and pay better attention to their talk. For programs that involved a formal presentation, either the talk was done while viewing the wolves (six programs) or given first (six programs). Perhaps reflecting this idea of wolves acting as a "carrot" is why I only heard about one program 
where wolf viewing preceded a presentation. Spencer explained, "You know, we want to do our talk, teach people our mission and the importance of wolves. And then we'll bring in the wolf. It's kind of like we're dangling that carrot in front of them." Likewise, Rachel explained how groups came and did service projects "and then at the end of the day, they get to meet the wolves, kind of like their reward."

\section{Benefits for Participants: Discussion Points}

All the educators believed that there are unique educational opportunities when visitors are able to view or howl with wolves. They reasoned that this is because visitors are able to make connections through experiencing an actual animal in the here and now, with their senses fully engaged. Research that specifically investigates the benefits for visitors when exposed to wolves is scarce. There is, however, some research looking at the educational value of zoos and aquariums, although little of it focuses on the value of viewing and experiencing actual, specific animals. In their research at one zoo, Mony and Heimlich (2008) found that participants reported receiving the message that animals are beautiful and have value more clearly through viewing animals in a naturalized habitat than through reading signs or talking with docents. As well, a large study conducted by Falk et al. (2007) found that 11 months after visiting a zoo or aquarium, close to half $(42 \%)$ of the participants remembered viewing a specific animal or species as the highlight of their visit; unfortunately, this does not speak to what, if any, learning that took place, although it does corroborate the wolf educators' assertion that seeing a live animal has an impact.

Seven of the educators specifically mentioned being able to directly observe wolf behaviours as being important. Ballantyne et al.'s (2007) review of zoo and aquarium 
research loosely supports this assertion. In that paper, the authors discussed studies that have shown the experience of observing animal behaviours can positively enhance people's attitudes towards conservation and/or their knowledge of the animals. As well, Ballantyne et al.'s review of research also led them to propose that there is sometimes an emotional connection made by visitors when viewing live animals, which supports the assertions made by eight of the educators.

There is also some related social science research that corroborates the position of the educators who argued that exposure to actual wolves can have a positive impact on visitors, but this research was not conducted in the context of education. For example, one study in Norway found that when a carnivore species (one of which was wolves) resided in a region, rural people in that region had less fear of the species than those in regions without, although it must also be noted that this did not necessary correlate with a more positive attitude towards the species (Røskaft, Bjerke, Kaltenborn, Linnell, \& Andersen, 2003). Similarly, Houston et al. (2010) found fewer negative stories about wolves appeared in the news in areas with permanent populations of wolves and where people had a history of living with them compared to areas where reintroduction or possible reintroduction was occurring. This loosely supports those five educators who suggested that the experience of observing wolves helped visitors understand that wolves are not "that bad," that is aggressive and dangerous to humans. Other research, however, has found that this is not always the case that experience with wolves reduces fear or negative attitudes. For example, numerous studies indicate that people who live in proximity to wolves tend to be more negative about them (Ericsson \& Heberlein, 2003; Karlsson \& Sjostrom, 2007; Williams et al., 2002). That being said, some of these 
researchers point out that this negativity might not be related to direct encounters with wolves, but about what wolves represent (Ericsson \& Heberlein, 2003; Karlsson \& Sjostrom, 2007).

\section{Wolves in Education: Ethical Aspects}

The educators had a lot to say when it came to discussing the ethical aspects of education involving captive and wild wolves. They talked about the ethics of having or not having display wolves socialized and comfortable with humans, the pros and cons of programs that have a "wolf on a leash," the importance of putting the wolves first, and the ethics of allowing wolves and visitors to interact.

Wild or socialized? There seemed to be two general positions when it came to the socialization of "ambassador" or "display" wolves. ${ }^{6}$ Ten of the 17 educators worked at programs where the wolves were socialized to some degree, at least with staff (see Figure 11). These ten educators agreed that the best way to socialize wolves was to have them bond with humans at a very young age. For example, Monique explained when they get new wolves at the centre, they try to get wolves as very young puppies, "12-14 days of age is our preference, during the neonate stage." She described to me how wolf fear-avoidance begins earlier in wolves than dogs and what happens if the social bonding with humans does not begin early: "If you don't interact with them [as puppies], by the time they're adults, you've got a public display that has 35,000 people visit it, they're hiding or they're pacing or they're not really out in the front." She felt that in the case of captive wolves such as the ones she worked with, it was better for the wolves to have

\footnotetext{
${ }^{6}$ For the four programs involved in the Species Survival Plan (SSP), the wolves in the program were kept as wild as possible in case they might be reintroduced into the wild. These wolves were usually "off display."
} 
them comfortable around people. Other educators also felt that having the wolves socialized allowed them to be less stressed. Spencer said, "Having these wolves in the public eye all the time, [socializing them], it's going to decrease their stress." Similarly, Shelley explained, "So we lessen their fear of humans so they're not pace-y and apprehensive and nervous all the time. So that they don't mind people visiting them.”

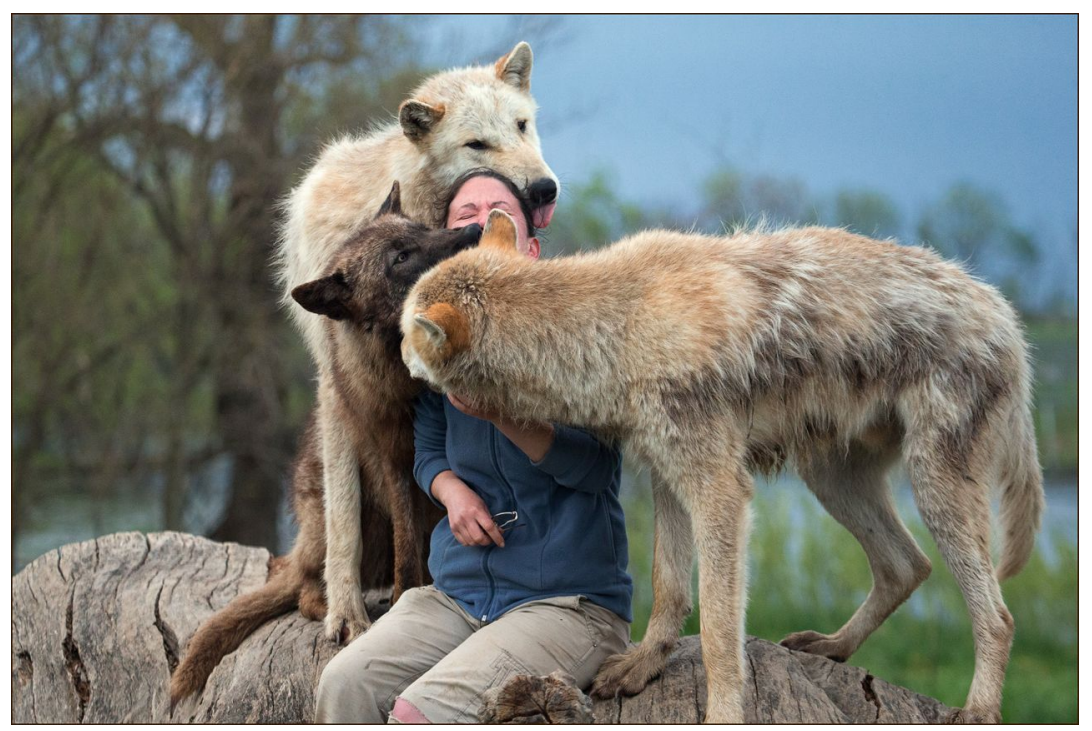

Figure 11. Socialized wolves with handler at Wolf Park. (C) Monty Sloan

These educators also felt that visitors would gain a more realistic perception about wolves if the observed wolves were not stressed and thus comfortable enough to display behaviours other than fear-avoidance ones. Put simply, Jeremy stated, "From an education standpoint, that's great, because the wolves will come into close proximity to people and the wolves feel comfortable and they will behave naturally." Likewise, Monique explained, "The last wild wolf I saw was not pacing or chewing on his tail, or making circles. I mean that's not how wolves act in the wild."

On the other hand, six educators worked at programs where the wolves were kept as wild as possible, with almost no human-wolf contact except for occasional health care or veterinarian visit care. Some of these programs even actively worked to maintain the 
wolves' fear and avoidance of humans. For example, some programs scheduled breaks or closed the centre or program for entire days so the wolves would not become accustomed to visitors. Other programs locked the wolves in separate areas when the display enclosures were being cleaned and one educator described their policy of never touching the wolves or wolf hybrids when cleaning enclosures even if the animals had come from homes where they had been socialized to humans.

These educators also justified their position on ethical grounds. For example, Gwen argued that they needed to "respect the inherent wildness of the animals that we have here. And allow that to be part of the education and the display of the animals." Recognizing that being less socialized to humans could cause wolves stress when encountering visitors, Christina explained how they made sure they had large enough enclosures: "So we just want to make sure that if they're ever nervous during a tour they have somewhere to go off exhibit. So the habitats are fairly large, [and] the whole habitat isn't on exhibit for tours." Despite the fact that the wolves at these centres were less socialized to humans than those in the other centres, the educators felt that their visitors were gaining a realistic idea of the wolf since wild wolves are naturally afraid of people.

Wolf-on-a-leash. For five of the programs with socialized wolves, "ambassador" wolves were sometimes taken off site (e.g., to visit classrooms or nature centres). Five educators who worked at places that did not offer such programs discussed either why their centre/program chose not to do so and/or the problems they saw with this approach. For example, Monty explained how in the early days, the centre he works at did offer programs involving wolves on leashes, "but they realised that they were giving the wrong message in many cases because people were coming away with the thought that, 'I want a 
wolf too.'... because they're seeing a wolf on a leash in the classroom, going, 'Wow, this is great. I want to do that." "Likewise, Erik discussed how because one of their organization's key messages was that wolves do not make good pets, they do not want to send mixed messages. He explained, "It's one thing to say, when you have a wolf on a leash, 'Wolves don't make good pets', but at the same time you're portraying them as a pet on a leash..., the message gets kind of mixed up."

It is important here to state that four of the five educators who expressed concerns about programs featuring a wolf on a leash often added the caveat of not wanting to judge others' choices. They mentioned how they knew of programs who did it well, or how it was simply a different choice, or just not the message their program wanted to share. For example, after explaining why his program didn't do "wolf-on-a-leash," Erik said, "Not to say that the places out there that do that are wrong, or they're not doing good work, it's just that Wolf Haven, with it's specific mission, doesn't lend well to doing ambassador programs."

Two of the five educators involved in wolf-on-a-leash programs discussed some of the same issues as well, and what they did to combat the contradictory messaging. For Spencer, not allowing the participants to touch the wolf helps reinforce the understanding that the wolf is a wild animal. He explained how their ambassador wolf does not interact with program participants at all: "I think people come out of that with a better understanding that these guys are wild. Just by him walking around and not really wanting to interact with people."

Leyton described how he and his staff addressed the issue head on by discussing it at length with participants. Still, he told a story about a passerby who took a photo of 
their ambassador wolf on a leash but had not heard their presentation: "The girl did not have a clue why my assistant was in the public with a wolf because all she saw was the wolf. She got enamoured and she took a picture; she walked away and she never got the message. So that's a big disservice." He explained at length why it was so important that people actually hear the talk in wolf-on-a-leash programs:

If I do not get to actually say what I need to say to each and every person, I've done a disservice. Because all they can see is there is a dude with long hair, tattoos, and earrings that has a wolf on a leash, so it must be a pet... Then if I don't get a chance to actually say, "No, no, no, no, you don't understand! I've rescued 400 in the last two decades. Six have been able to come on a leash in the public, those are bad odds."

Putting the wolves first. Whether it concerned wolves in a wolf-on-a leash program or display wolves housed at the various centres, seven educators discussed ways that they tried to put their program's wolves' needs first. The most common idea expressed in that regard was that the wolves were not there for the entertainment of visitors. For example, Noel said, "I know that there are a lot of people who would prefer us to use them for entertainment, you know, make them do tricks, make them do this and that, whenever [the visitors] please, but that's not the facility we are."

I heard how at a number of centres the enclosures were large enough that occasionally visitors would come and not actually see a wolf. For example, Jeremy told me that, "Sometimes we take people and we sit them there for half an hour, 45 minutes, waiting for wolves, and we never see a wolf. It's just part of what we deal with because we're never going to force the wolves to see people." Two educators explained how if visitors are disappointed and complain that the wolves did not "perform" or appear, they simply return the money paid for the visit. Other ways educators described putting the wolves first included the following: 
- Not procuring wolf puppies every year simply because they are popular with visitors, but rather committing to each animal they have in their facility for life.

- When it becomes clear young wolves are not comfortable with travelling, not pushing them to become ambassador wolves. Spencer explained, "they just weren't comfortable. And so, they make the decision by themselves that they didn't want to do that. So, that's their choice."

- Not allowing children to be rowdy near the enclosures and not allowing people to howl at the wolves. As Gwen stated, "Having people constantly making noise at the animals all day could create a stressful environment for them." Likewise, Rachel said, "If you think of it from the wolves' point of view, they are in detention while everyone else is at recess, and running around and playing. And it gets the wolves all worked up, you know, they get pissed off that they're inside these fences and can't run around and play with everyone else."

Ethics of taking people in with wolves. Two educators worked at programs

where general visitors could interact with the wolves, and three other educators worked at programs that had specialty opportunities that allowed visitors to go into the enclosures with the wolves or to go for a walk with a wolf. For the five educators involved in the programs that allowed interaction of one sort or another, the safety and ethical aspects of allowing visitors to interact with wolves was a topic of discussion. Most importantly, they felt that it was imperative to give visitors instruction about how to behave around, and interact with, the wolves. For example, Rachel had visited a different program where large groups of people were taken in with the wolves with little or no instruction. She said, "That's definitely one of the most unethical I think [I've seen]..., just not teaching the visitors that you're taking in with the animals how to interact with the wolves." While she explained that doing so was important for the safety of the guests, she also stressed that if there was an incident, the wolf's life might be at stake: "You know, if anything were to happen to any of these visitors, you know, that means pretty much instant death for the animal." 
Other. Finally, when it came to discussing the ethics of using live wolves in education, there were a variety of less common ideas that came up, as well as ideas that were discussed only briefly. For example, some of the educators talked about the ethics of breeding wolves in captivity. For at least five educators, having their wolves spayed or neutered so as not to contribute to more wolves in captivity was an important ethical choice. For three others, it was important to them that their wolves on site were ambassadors for wild wolves only because they themselves could not safely be in the wild. Jeremy discussed the cost to the animal of living captivity and how they should be given the best care possible. He went on to say, "I would never approve of this organization going and purchasing wolves from a breeder because that's just making the situation worse by putting more wolves into captivity."

In a different vein, three educators who worked at programs where participants were taken to hear wild wolves howl raised other matters. Rick, for example, discussed ethical considerations of these types of programs. While he felt that the howling program might cause some minor disturbance for the wolves, he suspected these were minimal. One reason was that, despite his howling with these packs multiple nights in a row, usually the disturbance was not enough to result in the wolves moving locations. He said, "We know that we can howl at them all four nights in a row, and they don't seem to move and don't seem to be impacted by this." In fact, he explained how sometimes they appear to stay in the same area throughout the whole month of August when the programs run. He went on, "We have the ability to meet so many people, and to educate them on the value of wolves. We think this far outweighs any concerns we have had related to having a disturbance on them." 


\section{Ethics of Wolves in Education: Discussion Points}

When it comes to education involving captive or wild wolves, for most of the educators, ethical matters loomed large. In some cases, the educators seemed concerned primarily about what was most ethical for the wolves involved (e.g., were they comfortable being viewed by visitors or was howling with wild wolves impacting them negatively?); in other cases their concerns were more visitor-focused (e.g., what messages were being conveyed to visitors, like when seeing a wolf on a leash or when viewing nervous and anxious unsocialized wolves). In some instances, concerns were about what was best for particular individual wolves on site (e.g., not allowing participants to be rowdy near the enclosures) while other concerns focused on what would ultimately help wolves more generally (e.g., not purchasing wolves from breeders thus not supporting the practice of breeding them for captivity). It was clear that most of the educators I interviewed had contemplated the ethics of education involving wolves and I believe this is a fairly notable finding - that wolf educators have deep concern about both the lives of the wolves they work with as well as the implications of their educational messaging.

There is some literature that considers the ethics of live animals in education. Warkentin (2011), for one, discusses the ethics of encounters that take place at "swimwith-the-dolphins" programs and I believe there are some parallels between these and the visitor-wolf encounters described by some of my participants. During her research, as a participant-observer Warkentin observed varying degrees of interspecies (i.e., humandolphin) etiquette being stressed. It varied substantially by program, which led her to encourage people to seriously consider the ethics of these types of human-animal 
encounters. As demonstrated by the discussions described above, most the educators I interviewed had seriously contemplated the ethics of the human-animal encounters that take place at their respective programs, which is encouraging.

Specifically, when it came to the question of having captive wolves remain as wild as possible or socializing them, educators arguing either position used ethical justifications. One the one side, I did find literature supporting the educators who felt socializing their facility's wolves to humans was preferable. Claxton (2011), in her review of research on improving the welfare for zoo animals, concluded that the evidence shows socialization to potentially contribute to animal welfare as a form of enrichment and in making the animals easier to care for, and it also has educative potential for visitors. On the other side, I could not find any literature related to keeping captive animals as wild as possible, although there is more general discussion in the literature about the ethics of keeping and displaying wild animals.

On this point, considering that 16 of the 17 educators worked at facilities with captive wolves, I found it interesting, but not all that surprising, that none of them directly discussed the ethics of having people pay money to view wolves who are captive and confined. (The exception was two educators who told me that visitors would occasionally complain that the wolves were in captivity.) Even though facilities with captive animals such as zoos and aquariums are likely to promote themselves as conservation-focused in today's world (Ballantyne et al., 2007; Falk et al., 2007), it is clear that many people are still visiting them for recreation and entertainment (Ballantyne et al., 2007). While some of my participant educators took great pains to stress that their purpose in having captive wolves was not entertainment and others pointed out how they 
only rescued animals who would not otherwise have a home, nonetheless none raised the possible hidden or implicit curricular messages of having people pay money to view captive wild animals.

Both Pedersen (2010) and Lloro-Bidart and Russell (2016) discuss some of the underlying or hidden curricula when places like zoos and aquariums display wild animals. For example, often the animals on display are not presented as subjects and/or individuals, rather as representations of their species. Lloro-Bidart and Russell write: "There is very little attention to them as individual subjects of their own lives; rather, they act as representatives of their kin or their ecosystems, martyred in the name of conservation" (2016, in press, p. tba). Both also note that often the justifications for having captive animals are instrumental (i.e., education, recreation, species preservation), overshadowing the fact that the animals themselves "are denied a life in freedom" (Pederson, 2010, p. 69). Pedersen also explores some of the hidden messages around the physical design of enclosures and how "the setting is at the same time an effect of, and helps produce, arrangements of visually oriented power relations manifested by humans over animals" (p. 80). In my discussions with the participants concerning the ethical aspects of wolves in education, none of these hidden messages were explored.

\section{How to Care for Captive Wolves}

I turn now to another common topic of conversation during the interviews: how best to care for captive wolves. It was clear that the educators, especially those who spent a lot of time caring for the wolves at their centres, were invested in and had firm ideas about wolf care as evidenced by the many coded passages from the interviews that seemed to fit best under the category of "how best to care for wolves." Quite simply, the 
main finding here is how important it was to these educators that captive wolves be well cared for. Indeed, these educators seemed to feel a keen responsibility towards the wolves in their care. For example, when asked what has kept her at the centre for 28 years, Monique replied, "Well, you know, you still feel like you're doing a service to the animal... I always try to tell people when you do this job, it's not about the humans, it's about the wolves. And the day that it becomes about a human over a wolf, then that's the day I need to leave.”

While the "how" of caring for wolves took up a fair bit of interview time, the insights that emerged out of this discussion took us a bit off topic from education. Still, it was often during their time caring for wolves that the most profound educator-wolf relations developed, which I will discuss in the next section. In the interest of brevity, I will just briefly touch on the main categories under the topic of wolf-care: enclosures, keeping wolves in pairs or packs, diet and feeding, breeding, socialization, veterinarian and health care, retirement, and ideas about how to foster happiness among captive wolves.

Enclosures, Packs, and Diet. Eleven of the educators discussed how their wolves had large or naturalized enclosures (see Figure 12) and I frequently heard from the educators that the wolf habitats at their wolf centres were much better than what would be found in a zoo.

The enclosures were usually occupied by male/female pairs, packs, or a mix of the two (i.e., some packs, some pairs). Four programs used the male-female pair model because, according to those educators, it is very difficult to create packs in captive settings, especially with adult wolves. The educators also explained how wolves, being 
social animals, needed to be housed with other wolves. On a few occasions, a particular wolf needed to be housed alone and the four educators who mentioned this outlined why it was necessary and how they attempted to enrich this particular wolf's life.

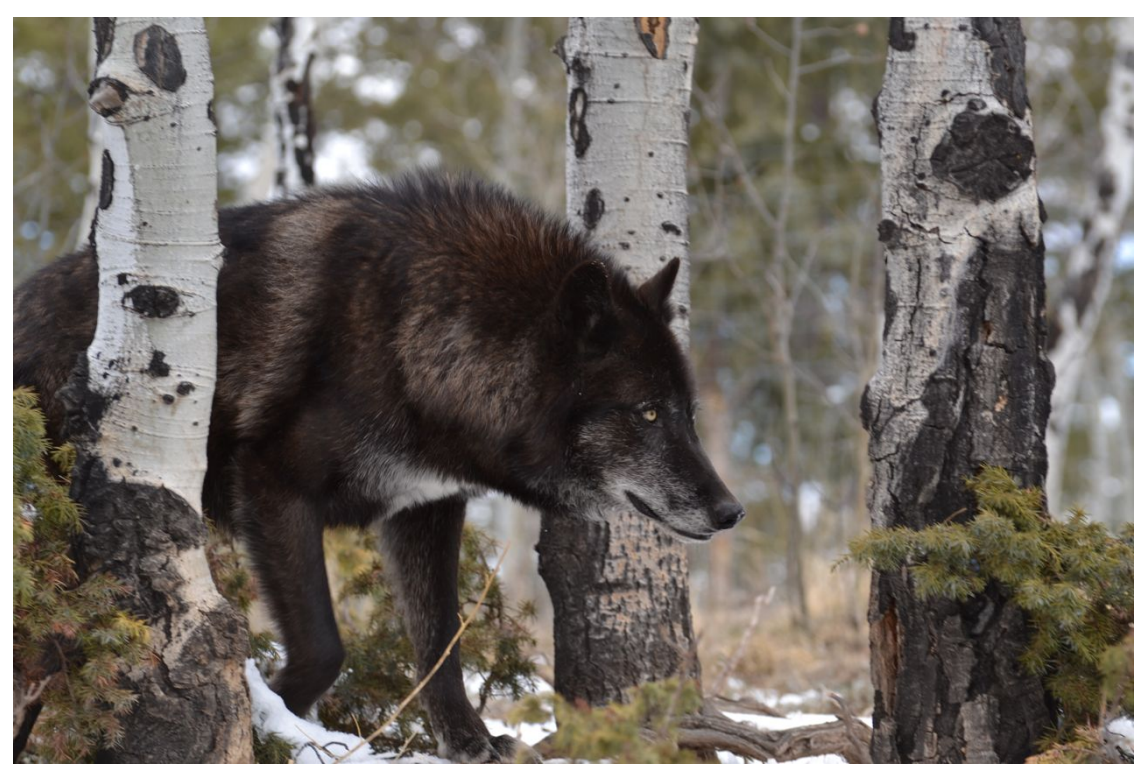

Figure 12. A wolf in a naturalized enclosure. (C) Michelle Smith

Some of the educators talked about diet. For example, I heard how two programs fed or supplemented wolves' diets with a kibble. Alternatively, eight educators reported how they either tried to feed wolves something closer to what their diet would be in the wild and/or on a schedule that would match the typical fast-famine diet of wild wolves (i.e., eating once every few days). These educators discussed the game meats they would use as food, such as deer, elk, and beaver. Sources of meat included roadkill and donations from local hunters and trappers.

Health care. Nine educators talked about health care and/or vet care. One described being involved in rounding up unsocialized wolves for vaccinations and four talked about administering drugs or vitamins to wolves (see Figures 13 and 14). I observed one centre's morning wolf care session and watched staff offer the wolves treats 
that masked drugs and vitamins. While observing, I suspected that administering health care to socialized wolves versus unsocialized wolves would be easier and less stressful for both the wolves and the staff, and the stories below supported that.

I heard quite a few stories about the ethical and decision-making aspects of delivering health care to wolves. For example, Jeremy told me a long story about how an alpha wolf was treated for a kidney issue while still in the enclosure with his pack. While Jeremy discussed the danger to the alpha wolf - the pack might have turned on him while he was being treated - he argued how his decision to treat within the enclosure also took into account not wanting to disrupt the structure of the pack because, "If we were going to do that with him, when we put him back with that pack, he very well could lose his rank." Similarly, Monty went on at length when describing a system they had devised to give wolves eye-drops after surgery, trying to stress the wolves as little as possible. They figured out that their wolves like a particular type of sausage: "They absolutely love cheesy brats," so they would feed them small pieces while the wolves were standing against a fence so that they could more easily stabilize the wolves' heads while the drops were administered.

I also heard one story about a decision not to treat an unsocialized wolf who had a run-in with a porcupine. In this case, the staff decided it was most ethical to not treat her, thereby allowing her to be more wild and not stressing her or the pack through human contact. Jennifer explained, "We just decided to monitor her closely because [a run-in with a porcupine] would happen in the wild, and in the wild they would have to figure it out." She explained how the quills eventually worked their way out and were gone in ten days. She continued, "The alternative is we tranquilize her, which is pretty dangerous, 
it's quite stressful. The rest of them have to watch us do this. So it was much better in the end."

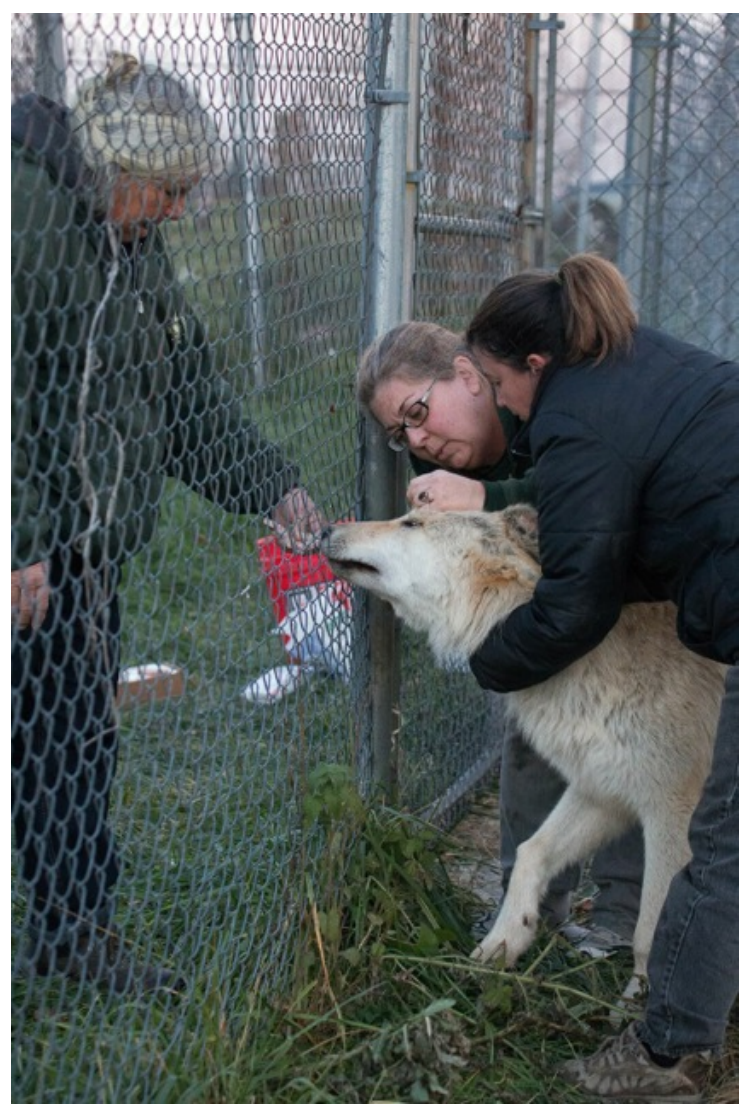

Figure 13. Administering eye drops. (C) Monty Sloan

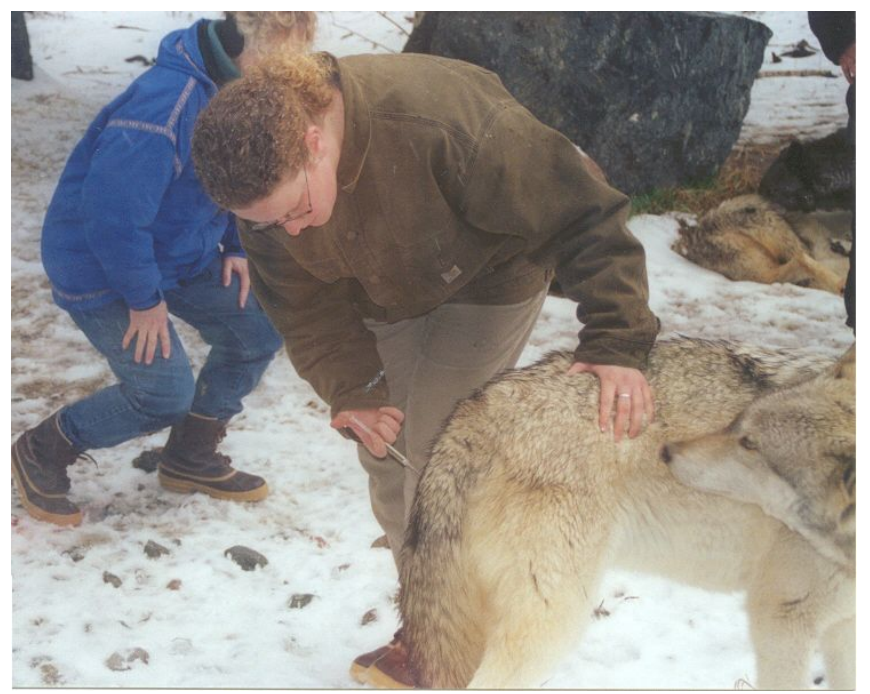

Figure 14. Health care with socialized wolves. (C) Wolf care staff 
Committing to wolves for life. Seven educators talked about "retiring" wolves and/or the importance of committing to wolves for their entire life. For instance, Jeremy discussed his centre's aging wolf pack and how taking on a new pack is not a decision to be made lightly: "It's a big commitment and endeavour if you're going to get more wolves. Because when you sign on to that, you're signing on for the lifespan of those animals, to do it properly. So, that is, if you get young animals, you're looking at a 15year commitment." For some, committing to wolves meant at some point retiring them (i.e., taking them off display). Monique explained, "So ethically, I believe in committing to an animal throughout its entire life. And that when the time is such that the display is overwhelming to an older animal, which for us is around ten years of age, that they have a right to exist without public viewing..., we're still obligated to that animal for the rest of its life."

Monty talked about the effort involved in caring for older wolves. For example, he discussed one wolf who was fed a special hand-mixed diet. He also discussed how because the wolves at his centre were so well socialized that they could do hands-on care of older wolves longer than some other places:

You know, as long as they have a good quality of life, they enjoy people, they can get up and move around, we'll keep them alive past the point where a lot of facilities would probably just euthanize the animal because it's either too difficult or impossible to work with them. Imagine a non-hand-raised wolf and if it's not eating well, there's nothing you can do for it. Whereas we can go in there and if an animal becomes incontinent, we can literally clean them up every day if necessary, and that will happen.

Keeping wolves happy. All of the educators involved in wolf care seemed to want the wolves to be happy, and six stated this desire explicitly. For instance Shelley said, "With the wolves themselves, you know just making sure that they're happy is 
probably the biggest challenge, and making sure that they're comfortable all the time." Ideas for keeping wolves happy included stimulating and enriching their lives with walks or outings, toys (see Figure 15), and interesting foods such as melons (see Figure 16). I learned about one centre that put fish in the enclosure pond (see Figures 17 and 18) and another that had an extra large enclosure or "day spa" where they would take the wolves to explore/recreate.

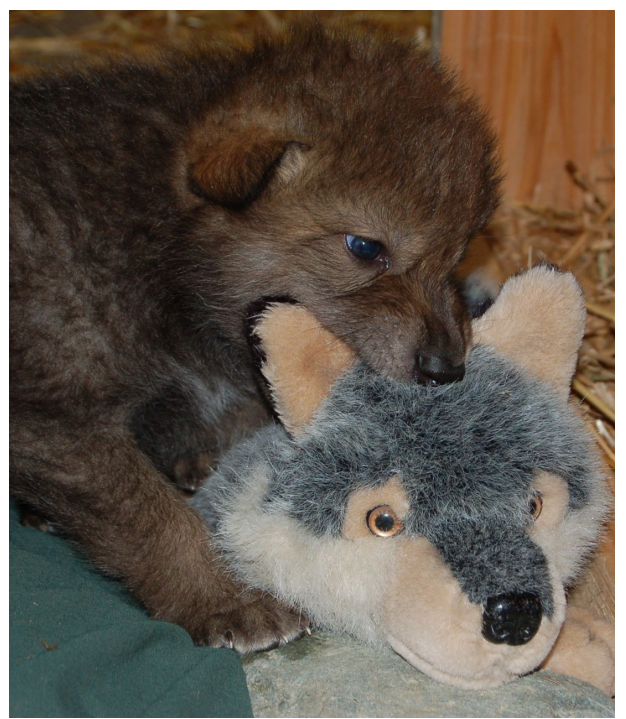

Figure 15. Wolf puppy with stuffed toy. C) Wolf care staff

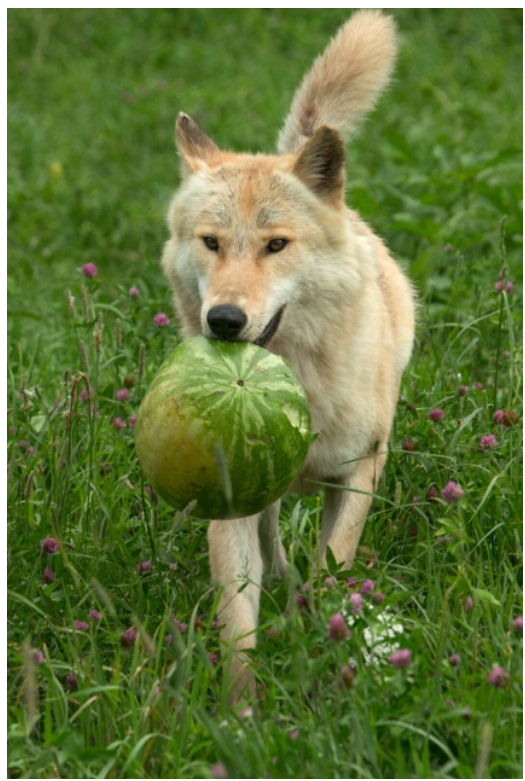

Figure 16. Wolf with watermelon. (C) Monty Sloan 


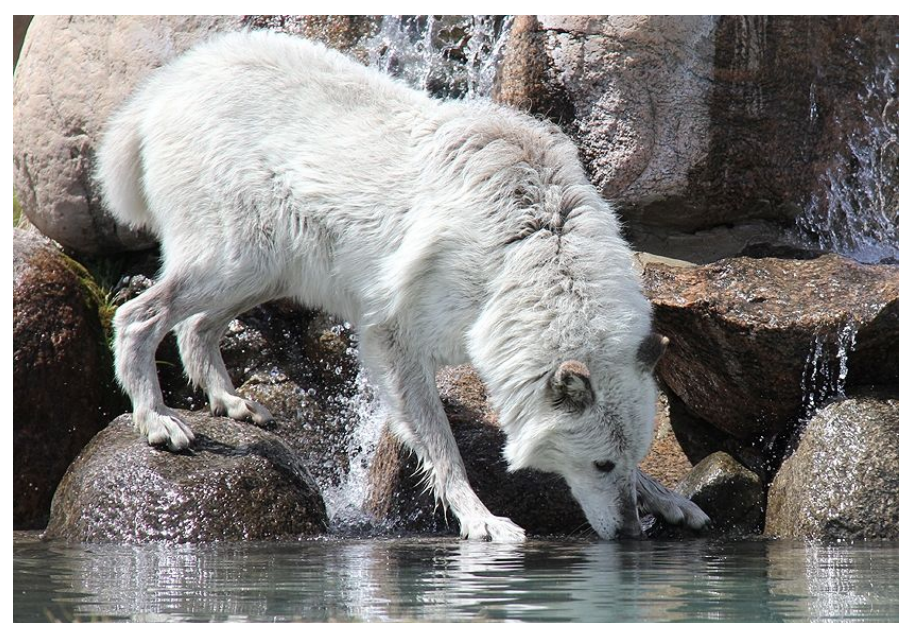

Figure 17. Wolf at an enclosure pond. (C) Courtesy of participant

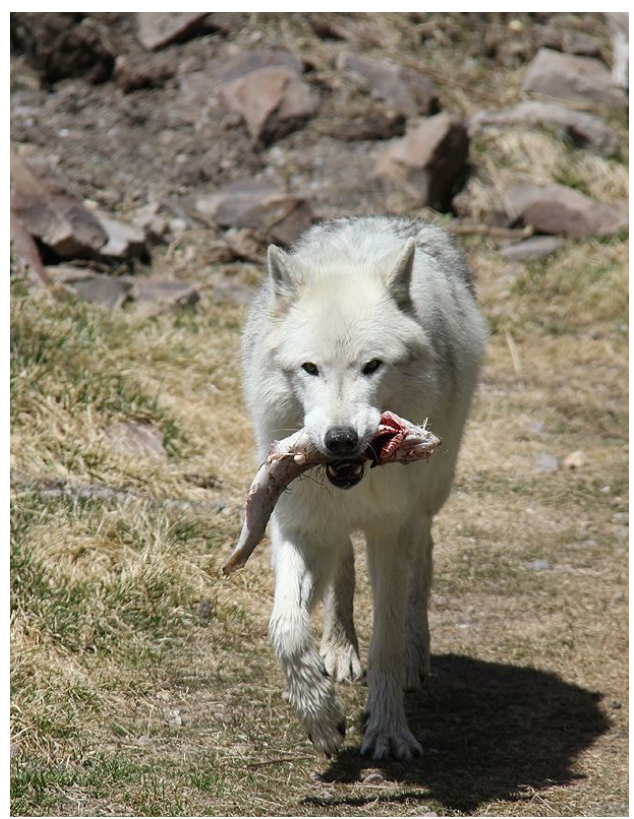

Figure 18. Wolf with fish from enclosure pond. (C) Courtesy of participant

\section{Wolf Care: Discussion Points}

I did not delve too deeply into the literature on animal care in zoos and aquariums as it diverges from my research question significantly, and I was more interested in the educator-wolf relations developed than the details about care. However, discussion of animal care including enclosures/habitats and creating enriching environments does come 
up in Ballantyne et al.'s (2007) review of conservation learning in zoos and aquariums. Supporting some of what I heard from the educators, they found that large enclosures have become more common in modern zoos: "These third generation exhibits use natural vegetation and landscaping to create the illusion that animals are living in their native environment" (p. 369). Similarly, both Ballantyne et al. (2007) and Claxton (2011) note that creating enriching environments for captive animals, as described by the educators above, is a fairly common practice in today's zoos and aquariums.

\section{Elements of Educator-Wolf Relationships}

While I have already discussed what the educators learned about wolves through interaction and observation, in this section I discuss particular elements of their humanwolf relationships such as the reciprocal and individual nature of educator-wolf relations, forming bonds, building trust, nurturing, and interspecies communication. Ten of the 17 educators had hands-on relationships with the wolves at their various programs. (I also include in this discussion an eleventh educator who spent half his time on wolf care and in enclosures, but as per the centre's policy did not actually touch the wolves.) Sometimes these 11 educators interacted with the wolves to maintain social bonds for health care reasons whereas in other cases interaction involved training and socialization of wolves so they could tolerate viewing and/or interaction with visitors.

I asked most of the educators during the interviews what the best part of their job or volunteer position was. For five of the ten involved in hands-on wolf care, working with and forming relationships with wolves was undoubtedly the best part of the job. As just one example, Rachel answered: "Wolf time. So my favourite part is being around the 
animals. Like, pretty much every single volunteer that comes is because we love wolves, and we want to work with them."

Relations are reciprocal and individual. The most common idea that emerged when discussing educator-wolf relations with these 11 educators was the concept that because wolves are individuals with their own unique personalities, each interspecies relationship was itself unique. In fact, all 11 described these interspecies relationships in this way (See Figure 19 for a visual representation of one of these relationships). It was also clear that the educators were discussing a two-way, or reciprocal, relationship. For example, Rachel talked about working with a group of wolves from the puppy stage on, explaining, "I love forming those relationships with an animal, and like watching how we both grow, learn how the other interacts." Monty noted, "There's some that you just have a better relationship with than others" and Jeremy explained, "It's sort of like wolves have individualities similar to humans, in the way that some humans you just never get along with, like oil and water... And wolves are like that towards humans too. Sometimes personalities [of] humans don't get along with the individuality of the wolves and so they just don't mix."

Eight educators shared specific examples of the unique relationships they had with particular wolves. For instance, Erik told about engaging in wolf care with another staff member, Barry (a pseudonym). In one enclosure there was a pair of wolves called Meeka and Lonnie. Meeka, he explained, seems to enjoy his company, but avoids coming near Barry whereas Lonnie likes him: “Meeka, the female, doesn't want anything to do with him, and she's the one in the back. But Lonnie will come like right up there." Erik thought it was really interesting "just to see how each wolf interacts with each person. 
Everyone has a different experience with the wolves, and so when you get those couple animals that seem to enjoy your company, you know that's really cool."

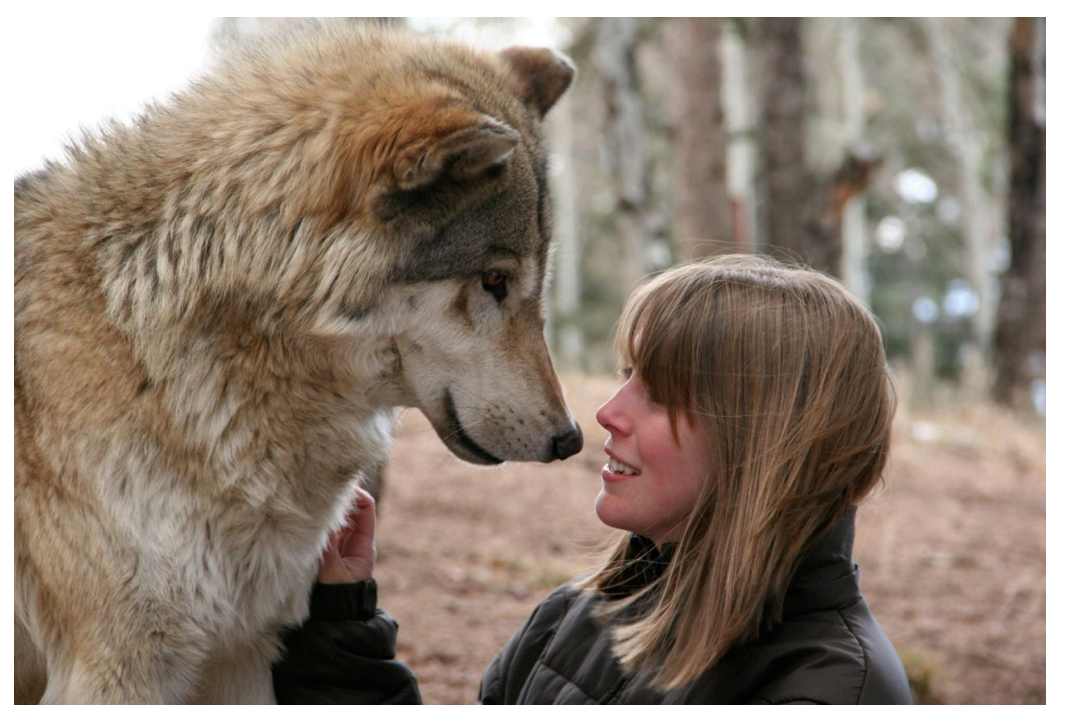

Figure 19. Michelle and Kwahadi, one of her favourite wolves. (C) Michelle Smith

Michelle described what happened when her relationship with a wolf named

Micah went sour. Micah is a socialized and usually friendly wolf with whom Michelle had a run-in. Micah remembered it and held a grudge. Michelle told me:

It's because he tried to take somebody's glasses, and I grabbed him on the nose like I do with Keyni all the time, because Keyni's still a puppy, and you can do things like that. And [Micah] dropped the glasses, but then he growled at me and he tried to jump on me, and tried to say that, "I'm the alpha still." It didn't work for him, but now he holds a grudge against me. Every time I try to go in there, he growls at me, gives me a little lip. So I'm like, "OK, I'll stay out."

Rachel discussed how the volunteers at the program where she worked formed unique relations with specific wolves or wolf hybrids (most of whom had been rescued), and how they often found a wolf with whom they especially connected. She stated:

The staff members find an animal that speaks to them, whether it's one of the shy animals, or one of the more outgoing animals, or one with a troubled past. They all, you know, wolves are a lot like people, they've all got different personalities, they have different stories, different backgrounds..., sometimes, you know, you just kind of click, like your personalities match with one of the animals. 
She went on to explain how one of her own favourite wolves is Daisy: "She's an absolute love. She's actually almost completely blind. And I'm also legally blind, so I kind of have a connection with her on that level... But, yeah, there are definitely a few that I like a little bit more than others."

Forming bonds. It was obvious that all the educators who interacted with the wolves on site had formed bonds with wolves (see Figure 20). For example, Jeremy said, "I guess one of the points is that the bond that wolves can have with people, when it's done right, is stronger than any bond I've seen with a dog and their owner." $\mathrm{He}$ elaborated, "You get attached to certain individuals, of course you do, especially when they're bonded to you. When they're socialized wolves, they're bonded to you." Similarly, Noel explained how their wolf care sessions each morning, where staff enter the enclosures, are partly conducted to maintain human-wolf bonds: "[M]aking sure that, you know, not only are we able to do a physical check-up on them, make sure they're physically healthy, but also to make sure that we still have a social bond with that animal."

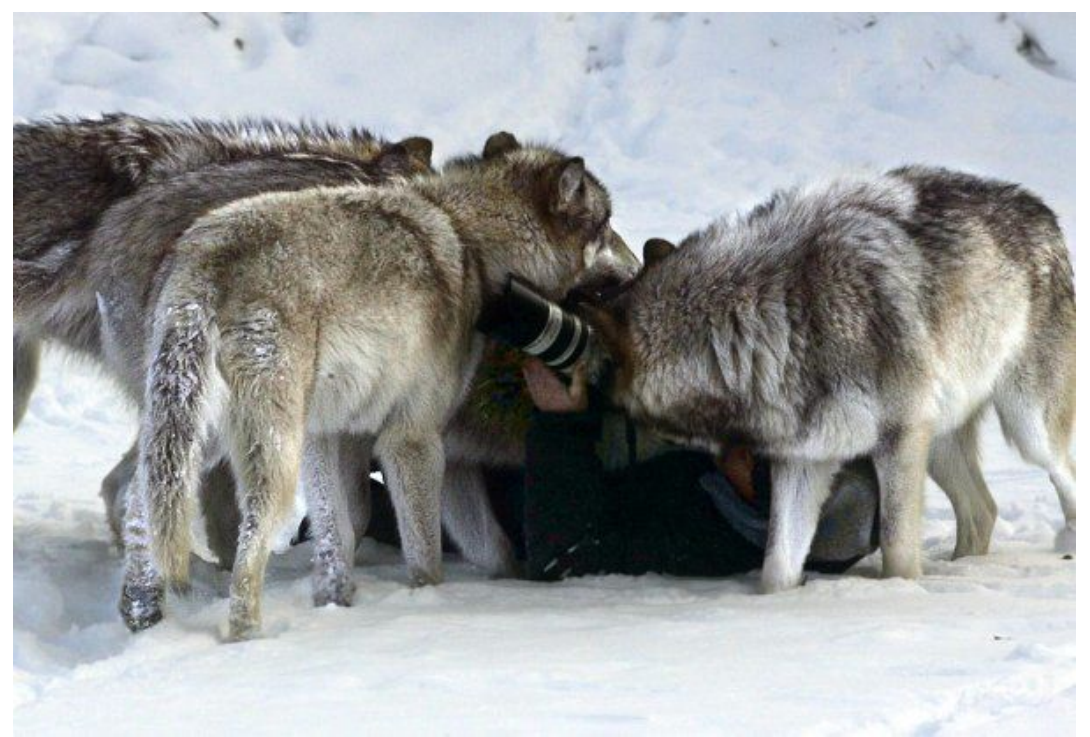

Figure 20. Monty photographing the pack. (C) Wolf Park 
Two educators explained how being bonded socially to wolves meant that it was hard for them when the wolves grew old and died. Shelley discussed a wolf named Maya, for example: "She's probably my favourite. I'll be sad, it will be one of the saddest days on earth when she's no longer with us." Likewise, having worked with wolves for so long, Monique explained how she had seen many die. She stated, "That's the hard part about wolves though. You know, they do live longer in captivity than in the wild, but like a dog, you're socially bonded to them, but they certainly don't live long enough to match our human [lifespan]."

I was lucky to observe a wolf-care session at one centre where staff entered the enclosure with a pack of wolves to do a health check and socialize with the wolves. Before the staff entered the enclosure, I saw clear evidence that the wolves were happily anticipating them, gathering together and waiting at the entrance. This corroborates the educators' ideas about the presence of social bonds. Here is a short passage from the observations I recorded in my journal:

After a few minutes, I see all four wolves move over to the fence. I see one yawn and twice do a "play bow" which is so familiar to me as I have had one or more dogs for years. It surprises me how all four wolves look so very excited (tails wagging!) as they are obviously anticipating the humans entering. I wonder how much of their excitement is in anticipation of these particular humans, whether they have a bond, or whether they might greet any human the same? I speculate that it's the former. I figure, like with dogs, wolves being social animals must form bonds with particular individuals. I'll have to ask about that later.

Later, while talking to one of the educators I had observed, the participant confirmed the presence of bonds built in part through trust, which I turn to next.

Trust. Four educators discussed how trust was an important element of the educator-wolf bond. I suspect a good number of the other educators who had relations with wolves would also agree that trust was important; either it did not come up or else 
they only alluded to it while discussing related ideas such as forming relations with individual wolves.

Noel described what a powerful feeling it was to have a relationship where the wolf was willing to trust him: "The fact that these animals will trust you is a really overwhelming feeling, to know that they're willing to trust you." He went on to tell about needing to draw blood from an unsedated wolf and how that was only possible because of the trust bond. Minutes after drawing blood, the wolf was happy to take food from his hand. He continued, "not even two minutes later, the fact that they are willing to trust us that much, it's amazing, it's a really good feeling."

Monique described how having that kind of trust with the centre's wolves came with a huge feeling of responsibility: "The wolves that are challenging for others to work with, who trust me enough to be interacting with them, that's the most rewarding part, and it's also the worst part because they trust me unconditionally. And that's the hard part." She explained further that because of that trust, she felt responsible for being an advocate for their needs. In fact, making sure she lived up to their trust was a big part of what kept her in the job. She continued, “They're ambassadors for the wild, I'm the voice for those ambassadors. I'm the one who says, 'Enough is enough,' you know? This is not how we're going to manage [them]. And so that's what keeps me here."

Monty described how some wolves at the centre trusted him enough that he could actually pick them up. Because he worked so intimately with these wolves, at one point he wondered, "What's the wolf going to do if I pick it up? They like being held when they're puppies, let's just try and see what they do as adults. If they trust you a lot, they're fine with it." He went on to explain how if a wolf becomes scared and shuts 
down or becomes aggressively aroused, "I can just pick them up and carry them off, which is really convenient."

Jeremy, too, described trust as an element of the bonds he has with the wolves on site. He told a story which demonstrates how this trust could go both ways, how his own safety was sometimes in "the paws or jaws" of the wolves:

I was interacting with the pack one day and we have a lot of flies around because there's a lot of wolf food around. And a fly landed on my forearm and the alpha male, who is, you know, you don't contest him, that's part of handling him, the alpha male gets to do what he wants to do, and if it doesn't involve human safety, then he is allowed to do whatever he wants. So, he eyeballed this fly. He's definitely zoomed in on this fly with his eyes and he was within strike range of me and I was like, "Oh no!" because I knew what was coming next. He's going to snap at the fly, and I'm thinking my forearm is right there. They obviously have intense pressure capabilities with their jaws - enough to snap any bone in our bodies. I'm thinking, "Holy cow, here goes my radius and ulna snapped in half here." So, I was just ready to reach for him, actually to swat the fly away is what I was trying to do. I was trying to get the fly off of my arm so he wouldn't snap at it, and he launched and snapped at that fly and he caught the fly, ate the fly on my arm, and the only thing I felt was his lips graze my skin very, very softly. And I was like, "Holy crap!" because it was a full-on snap. So, it's just they have really, really good co-ordination. And also, I mean when you think about it, why would he not just take a chunk out of my arm? A lot of that is the socialness of the wolves. They don't want to hurt us.

Nurturing. One important aspect of the relationship some of the educators had with wolves seemed to involve nurturing. Nine of the educators who worked at centres with socialized wolves discussed working with and/or raising puppies. When four of the educators discussed favourite wolves, they were wolves with whom they had a hand in helping to raise and nurture. For example, Spencer told me, "I won't say it too loud, but you know, I'm really sweet on Alawa, the three year old Canadian grey, or Rocky Mountain [wolf]. She was one of the ones that I helped raise." Likewise, when asked if he had a favourite wolf, Noel replied, "I think, of all we have, I think Luna's probably, she's probably the most tractable. I was here with her for most of her raising here at the 
centre, so we have chemistry, let's say that." As well, I noted that three of the educators referred to the wolves in their centre in a maternal or paternal manner, for example, referring to themselves as "daddy" or calling a certain wolf "my little baby" or the wolves "my kids" or "babes."

Wolf-educator communication. Finally, five of the educators talked specifically about wolf-human or interspecies communication. Leyton, for one, discussed it at length. He believed over the years that he had learned to communicate with certain wolves using a kind of language: "I'm not going to claim to be an animal psychic or an animal communicator, but I will tell you that they talk. And they do have a language system." He went on to discuss how he had learned this language system from a female wolf early on in his career, and had been able to communicate with certain wolves using it since that time. The other four educators who discussed communication discussed it in terms of how wolves pick up on human emotion (e.g., confidence, negativity, fear). For example, three educators talked about how participants, new staff, or interns were tested, picked on, or teased by wolves if they sensed fear or nervousness. Similarly, two educators stressed how it was important to put negative emotions and inter-human conflict aside when working with the wolves. For instance, Rachel said, "Actually the wolves pick up on it, and I've seen it completely ruin a person's relationship with the animals. Just because they have issue with somebody else [like a fellow staff member].”

\section{Elements of Educator-Wolf Relationships: Discussion points.}

Through caring for and socializing with the wolves, 11 educators had built humanwolf interspecies relations. All of them talked about a two-way, reciprocal relationship between two unique subjects who both had agency. Bonds had been formed, some 
stronger than others, and at least nine of the educators had taken part in nurturing the wolves. Some discussed the importance of trust between humans and captive wolves, and some discussed how they were able to communicate with wolves on some level.

It appears clear to me that direct experiences interacting with and forming relations with other animals can lead to unique learnings different than those developed in the abstract. To one degree or another, the experiences and subsequent insights of the 11 wolf educators here who had direct interactions with wolves support this. For one, when I compared the interviews of those who did or did not interact directly with wolves, those who had such experiences talked more extensively about the wolves they worked with and the interviews were often longer. As well, these educators seemed to have a lot more to say about what they had learned about wolves, often bolstering their assertions using specific wolves as examples rather than talking about wolves in general, abstract terms.

There is some research that supports the idea that direct interspecies interactions lead to unique learnings and understandings. For example, Ross et al. (2003) argue that experiences with nature, including other animals, can lead children to engage in more ecocentric rather than anthropocentric reasoning about nature. Such experiences can also influence how people interpret other animals' behaviours; for example in one study, those who grew up with pets were more likely to describe dog behaviours as involving desires, feelings, and understandings than those who did not (Light \& Costall; 1996). Similarly, in another study, children who had pets or farm animals at home typically had better attitudes and more knowledge about both popular and unpopular animals, wolves included (Prokop \& Tunnicliffe, 2010). And in the case of a study conducted by Fawcett 
(2002), even a short direct experience with a wild animal (in this case, a bat, raccoon, or frog) led kindergarten children to feel more positively towards that animal.

Along the same lines, there is mounting research showing that direct experiences with other animals, including pets/companion animals, working animals, research animals, or wild animals, can lead people to understanding them as subjects of their own lives and as having agency (Fawcett, 2002; Irvine, 2004; Kuhl, 2011a; MacPherson; 2011; Pedersen, 2011; Sanders, 1999; Watson, 2006). And while it is scarcely discussed in the literature, trust as an important aspect of interspecies relationships has occasionally appeared in research findings. For example, trust emerged as an element in Sander's (1999) investigation into the relationships between guide dogs and visually impaired people, as well as in my own (2011a) research that explored human-sled dog relations. In the case of my participants, their accounts of their experiences with wolves were related to me as encounters with wolves as acting subjects. Thus many of the possible elements that comprise relations with another sentient being, such as trust, seemed possible and relevant.

\section{Conclusion}

I have discussed two main themes in this chapter: understanding wolves, and working with wolves. I separated the two because, to me, there seemed to be a categorical difference between the interview data that related to how educators understood wolves on a conceptual level and all of the discussion and accounts they shared that were about, or arose directly out of, their interactions and concrete relations 
with the wolves with whom they worked. Of course, while I chose to separate the two, there is definitely overlap and connections between them.

Ultimately, this chapter has particularly addressed three of my five sub-questions: How has working with and teaching about wolves had an impact on participants' personal attitudes and beliefs about wolves? What do educators learn through working with wolves at wolf programs, both about wolves, and from the wolves themselves? And what ethical issues, if any, are raised for them in using captive or wild wolves in education programs? As discussed above, working with and teaching about wolves had an impact on the participants' personal beliefs about wolves, as well as their ethical beliefs and stances when it came to both wolf care, and educating with wolves.

One of the most salient points I believe we can draw from the findings and discussion in this chapter is the power of interspecies relations, and the importance of the insights and knowledge that emerge out of these relations. There is a tendency, in western society at least, to privilege various ways of knowing over others (Evernden, 1985; Russell \& Bell, 1996). When it comes to other animals, I propose that direct experiences with them are just as real and relevant as the "facts" generated about them from scientific research, especially when animal others are framed as objects (Evernden, 1985; Noske, 1997; Russell, 2005). Evernden (1985) writes, "How can we permit this reversal of the primary and the secondary, our own direct experience of the world and an abstraction about it which for most of us really amounts to secondhand information? Why is the gossip of experts more real than immediate experience?" (p. 78). The wolf educators' understandings about wolves and human wolf relations developed through concrete observations and relations, that is, through knowing actual wolves, deepens our 
overall understanding about wolves and could be useful in contributing perspectives to aid in improved wild wolf-human relations.

Further, the fact that some humans can have rich interspecies relationships such as these and come to know other animals as subjects with agency (as was the case for some of the participating educators) also raises interesting ethical considerations about how we treat other animals more generally. For the educators I interviewed, this was evident especially when we discussed the conservation of wild wolves. Some of them questioned the ethics of certain conservation management practices given what they had come to know and understand about wolves through the experience of working with them at their centres. This brings me to the next chapter, which is focused on the controversial issue of wolf conservation. 


\section{CHAPTER SEVEN}

\section{ENGAGING IN THE CONTROVERSY OF WOLF CONSERVATION}

Even in regions where it is less controversial, there is enough conflict surrounding wolf conservation in North America generally that all of the educators discussed how they understood and negotiated the subject. Indeed, there was so much discussion of the topic that "engaging in the controversy of wolf conservation" emerged as the final overarching theme. For some participants, dealing with the conflict around wolf conservation was one of the most difficult parts of the job. For instance, two educators involved in the Species Survival Program (SSP) shared their frustration when hearing about one of the wolves from the program who, after being released into the wild, had been shot illegally. Similarly, five educators discussed how they found the constant barrage of "bad news for wolves" hard to deal with. They cited examples like people bragging online about running over or shooting wolves or certain states dropping legislation that protected wild wolves. In this chapter, I discuss educators' experiences of responding to the controversy of wolf conservation, organized under three sub-themes: why educators feel wild wolves are so important; what they believe are the biggest barriers to wild wolf conservation; and their ideas about wild wolf management.

\section{Why Wolves Matter}

I asked all of the educators why they felt wolves and the conservation of wolves was so important in the first place. When discussing the importance of wolf conservation, three main ideas emerged: the importance of wolves for healthy 
ecosystems, their intrinsic value or right to exist, and the economic value of wolves for humans.

\section{Benefits to Ecosystems}

When asked explicitly for a rationale for wolf conservation, 13 educators talked about the different ways they benefitted the ecosystems. (Ecosystem benefits were also discussed in chapter five, but in the context of educational messaging rather than as a justification for their conservation.) Christina, for example, offered this rationale: "My biggest reason is how important they are to the ecosystem and how big a role they play in keeping an ecosystem balanced." Likewise, Ashley discussed the encouraging effects of

Red wolves being returned to the North Carolina ecosystem. She explained how they had impacted the sea turtle population positively due to the fact that the wolves were preying on raccoons who in turn were eating fewer turtle eggs. Similarly, Rachel talked at length about their positive ecosystem effects. She said, "We cover [in our presentations] why they're important in the wild. So the trophic cascades, the studies in Yellowstone, sharing, you know, how wolves help keep a healthy ecosystem."

I found it interesting that, like Rachel, 15 of the 17 educators mentioned Yellowstone National Park during the interviews, usually in the context of how wolves had positively impacted the park's ecosystem. For example, Jennifer explained:

All you have to do is look at the studies that are going on in Yellowstone Park and the effects that they are having on the grizzly bear population and the cold-water fish population and the plant community. I mean they're an apex predator, but they are really a keystone species that have an effect on everything right from the ground up.

Whether or not the participants' centres were located anywhere near Yellowstone, it appeared to be commonly used in their educational programming as an example. For 
instance, Shelley who is based in British Columbia told me, "We talk about Yellowstone National Park, the reintroduction of the wolf into their park system, and the devastation that happened with not having wolves for 70 years."

Five educators discussed the positive ecosystem effects wolves might have if they were able to return to areas where they had long been extinct. For example, Spencer explained that in parts of New York State:

Our ecosystem is screaming for the [wolf]. The overpopulation of deer, our forests are so unhealthy. Deer have overbrowsed, we have a lot of invasive species. You know, there's even more disease. And then we do have that large coyote, which the....wolf could help take care of that.

Similarly, Ashley said, "I think it's a mind shift, when people start thinking, 'Oh, if the wolf was here, they would fight coyotes for territory and the coyotes would kind of disperse, and there are way too many of them living on my land."”

\section{Wolves' Right to Exist}

After positive effects on ecosystems, the most common reason educators felt wolf conservation was important was because wolves had intrinsic worth and a right to exist. Six educators discussed this. For example, when asked why wolf survival is important, Spencer answered, "Well, first off, they deserve to be here. They were here before our settlers." Similarly, Monique responded:

I think, why not? I mean, who are we to say that any species who's been extirpated when that habitat exists doesn't have the right to live there, you know? I guess that's my first and foremost personal point of view. Who are we as a species to dictate, if habitat is available, that a species doesn't have a right to be there?

Ashley discussed that while wolves' intrinsic value was definitely worth considering, it likely would not be a convincing argument to use with wolf naysayers: "Some of the kids say, 'Well, because everything has a right to live.' Well, that's a great answer, but that's 
not going to make people who hate wolves want to have them around." She felt their benefits to ecosystems were more compelling when arguing for wolf conservation.

\section{Economic Value}

Another subject that was raised by five educators was wolves' economic potential. For example, when discussing the importance of wolf conservation, Michelle mentioned "the economic value" and noted that "wolves bring in tons of tourists and tourism dollars." These educators either focused on the economics of wolf-focused ecotourism or worked at centres that profited from wild wolf ecotourism by offering participants opportunities to see wolves in the wild through trips to places like Yellowstone, the Northwest Territories, or Isle Royale. For example, Spencer talked about how the New York Wolf Center, where he works, takes trips to Yellowstone, putting money into that local economy. He thought if wolves were reintroduced to the Adirondacks in his state, not only would it benefit the ecosystem, but it could benefit that local economy as well:

Now the Adirondacks, you know, that area up there, people are hurting for jobs. There's 18 million habitable acres. [The wolves] could actually have a very big impact on those economies. You know, more people would travel there. I know we would take trips up there as an organization, and money would then be spent in the restaurants and gift shops and farmers markets and things if we were up there. It's beautiful country. So I think it would help.

\section{Why Wolves Matter: Discussion Points}

All three of these arguments for wolf conservation are also discussed in research literature. At least since the 1960's and 1970's, and continuing into the present, biologists have been discovering and discussing wolves' positive effects on ecosystems (Eisenberg, 2010; Ripple, 2014; Troxell et. al, 2009). I suspect the fact that the educators cited wolves' ecosystem benefits in Yellowstone specifically is likely because the wolves there have been studied extensively since their reintroduction in 1995 and there has been 
substantial communication about this research in both academic articles (e.g., Ripple \& Beschta, 2004) and popular press books and articles (e.g., Chadwick, 2010; Eisenberg, 2014). The economic value of wolf-focused ecotourism is also cited in the literature (Fritts, et al., 2003; Ripple et al., 2014). And finally, wolves' intrinsic value and/or right to exist from an ethical standpoint has also been argued over the years (e.g., Bekoff, 2014; Fox \& Bekoff, 2009; Jickling, 2005a; Jickling \& Paquet, 2005; Lynn, 2007). However, despite the fact that there are a number of arguments for conserving wolves, obstacles to their survival persist.

\section{Barriers to Conservation}

When discussing the topic of wild wolf conservation in North America, often the educators also talked about the barriers facing wolf conservation. These fell into one or more of five key categories: human attitudes, misinformation, politics and legislation, habitat, and human-caused mortality. In this section, I also examine the issue of wolves being blamed or scapegoated (i.e., blamed erroneously for livestock deaths or declines in deer populations like those of elk or caribou).

\section{Human Attitudes}

Seven of the educators felt that negative attitudes and beliefs were the main obstacle facing wolves and their recovery and/or continued survival. These educators believed that if humans supported and wanted wolves on the landscape then wolf conservation would be successful. As Rick stated, "In the end, it's going to be about human attitudes — what's important to us as a society." 
At least four educators delved quite deeply into the underlying ways of thinking, or ideologies, at the base of human attitudes, ultimately arguing that in order for humans to live alongside wolves the way we think about nature in general may have to change. For example, Jennifer pointed to "the anthropocentric delusion where we think that we've got it all figured out and the world is ours. So, it's our thinking that makes us not open to sharing our space with wolves and other top predators." Similarly, I heard about how human arrogance can lead to problems. Monique, for instance, discussed how we cannot know the long-term ecological consequences of wiping out a species. Describing how in some places, in the absence of wolves, coyotes have started to fill that niche, she noted: ...for the most part, wolves have a fear of people much stronger than coyotes... So coyotes are going to [create] packs, join in a social relationship to capture larger prey, they're going to be bigger, and they're going to be more formidable and they're still going to be roaming the streets of D.C. I'm sorry, but that's not a good combination!

She moved to a discussion of coyote/wolf hybrids, explaining the problems they have caused since they "are not afraid of people. I mean, yeah, they're going after people, campers, and to me, that's the coyote gene. And that's something very dangerous. That is why you don't mess with Mother Nature! Wolves have their reasons for being what they are."

Also related to negative attitudes, three educators discussed how it was unfair to judge wolves using a human moral yardstick. They critiqued the use of words such as "vicious" when describing wolves and how humans are often blind to their own brutality. Michelle, for example, said, "You get online and you see all the pictures of elk that have been bitten and gotten away. So, of course, that looks bad, it's awful. But they're wild 
animals. How many times do hunters go out and they get a deer and they can't find it?

We don't demonize the hunter for that."

\section{Misinformation}

Closely tied to negative attitudes, ten educators felt that misinformation was a big challenge facing wild wolf conservation. Put simply, Michelle said, "You have a lot [of people] that are against wolves, and unfortunately, I think a lot of those who are against wolves, it's mostly the hearsay that they listen to." These educators argued that one reason people feel so negatively towards wolves and their conservation is that they believe false information and/or propaganda about wolves, which is sometimes being fed to them through anti-wolf groups. Educators also told me that some people seem to accept as true anecdotes or stories about wolves that are blown out of proportion or they believe the fairy tales, fables, and movies that portray the wolf as vicious and villainous. All of these can skew ideas about wolves according to these educators. For instance, when it came to misinformation, Matthew said,

[W]ithin the context of some of the misinformation that they're exposed to, either culturally through all these stories, or the misinformation that's out there, even just in the local area-some of it's purposeful, some of it's just ignorance about wolves. There's a lot of myths, there's a lot of ideas about wolves that just are incorrect, they're just not factually based, but they become ingrained in culture.

He went on to give the example of how some people believe that the wolf reintroduced to Yellowstone from Canada is a different wolf than what was in the area originally. Michelle shared the same example. Both Matthew and Michelle explained how this "fact" is just not true, and that wolves would have travelled throughout the region taking no heed of the political border. Matthew continued, "There would have been an interchange of genetics. So this idea that somehow the border between the United States 
and Canada is some sort of ecological boundary, [with] one type of wolf on one side, a different type of wolf on the other side..., it's just meaningless."

In a similar vein, Erik discussed how some people believe anecdotes told to them by their neighbours more so than scientific information gathered through research: "It's their personal experience... [The] general anti-wolf guy is going to trust Joe down the street who he's lived next to for years and years and years over what scientists are saying. So when Joe says that there's 200-pound wolves running around on his property, [he's] going to believe it." Likewise, Ashley said, "I'm learning... a lot of the reasons why people aren't supporting wolves in their areas, it's a lot of myths. It's a lot of 'my kid is going to get eaten at the school bus stop."”

These educators felt that as long as people bought such misinformation, support for wolf survival and protective legislation would be difficult to muster and activities such as illegal wolf killing would continue. One reason that four educators felt misinformation and negative attitudes were so rife in some areas like Yellowstone, North Carolina, and the southwestern United States was that reintroduction programs had not been adequately preceded by educational efforts. For example, Noel said,

I mean you don't reintroduce a large carnivore into an area and expect people not to notice... Not having that education there creates a lot of problems, it creates community issues. There are a lot of folks who poach animals in these locations because maybe they're misinformed about what the animal does.

\section{Scapegoating Wolves}

Quite often, the educators discussed how misinformation and negative attitudes led to wolves being blamed or "scapegoated" for things they do not actually do. For example, eight educators talked about how livestock (e.g., cattle, sheep) deaths and declining prey species (e.g., deer, elk, moose) are sometimes caused by other predators 
(e.g., grizzlies, wild dogs), weather events, disease, and habitat degradation. For instance, Spencer said, "You know, 26\% of livestock deaths come because of respiratory problems and bad husbandry techniques..., weather, grizzlies, dogs. You know, they fall in a hole and break their leg. But then so much is thrown at the wolves."

An interesting phenomenon I noticed in discussions of how wolves get blamed or scapegoated was that eight of the educators cited facts and statistics in response. The information usually pertained to wolves' prey, their limited threat to humans or pets, and the issue of depredations on livestock. For example, Leyton stated, "More cows die from lightning strikes and prairie dog holes than they do from wolf predation any day of the week" and "more humans each year die from a coconut falling on their head than they do from a wolf attack." Likewise, Michelle said, "I think as far as the livestock losses in the lower 48 [states] go, we actually lose four million animals every year, and out of four million, 3.8 [million] of those are from disease and weather." Also discussing livestock, Shelley explained, "Respiratory diseases and poisonous plants kill more livestock in western Canada than any other thing going. More livestock dies from transportation from the farm or the ranch to the slaughterhouse than dies in the field." Similarly when discussing an area of the United States where wolves are being reintroduced, Monty explained:

There's billboards up now about, "Here's what wolves will do to your dog!" and there's a picture of the remains of a dog that's been torn up and partially eaten. Well, yeah, that does happen, but your dog is much more likely to be killed by a car than a wolf. Your dog is much more likely to die of anything than a wolf. There's just a couple cases where wolves have killed dogs. 


\section{Politics and Legislation}

Eight educators talked about politics and legislation when asked about key challenges facing the survival of wild wolves. Some educators discussed how the politics of certain states affects how wolves are managed and protected. For example, Rachel said:

But politics definitely comes into play. Who has the money in their pockets kind of regulates where wolves are allowed to be living freely. So, like the Pacific Northwest, you know, Washington is pretty much the only state that does not have a wolf-hunting plan in state. And Washington is also one of the most liberal states in the country. So depending on the politics of the states and really kind of where the income comes from. So like Texas, never going to happen! Because of all the livestock that goes through there. It's just really a matter, unfortunately, of who has the most say in the actual making of the laws.

Shelley too argued that politics and money had a big influence on how decisions that ultimately affected wolves were made: "So, the anti-wolf guys are the guys that are lining the major politicians up, and saying, 'I don't want this to happen. So if you want my money, you're going to have to make sure this is going to happen for me."”

At the time I conducted this study, the United States had just recently removed the Grey wolf from the endangered species list in five Rocky Mountain states and was considering removing them on a federal level as well (Eisenberg, 2014; Treves \& Bruskotter, 2011). These decisions were something the American educators discussed frequently during the interviews. For example Christina said:

Right now, it's probably one of the most controversial things in legislation..., whether or not they're going to be de-listed from the United States federal Endangered Species Act. It's really controversial and heated on both sides. Lobbying groups have a lot to do with it in the political sphere. So that is kind of a pretty big thing that wolves have to deal with. 


\section{Habitat}

Seven educators argued that habitat was a key issue facing wild wolves. For example, Monique stated, "If I looked at habitat, to me that's the bigger problem. It's development." Indeed, she argued that the people who were fighting against each other over different types of protection and management of wolves should actually band together to fight the bigger issue of wildland preservation:

The people that want to trap wolves and hunt wolves, and the people who want to save wolves and love wolves, we should all band together. Because there are plenty of oil companies and gas companies and mining companies that are taking up wild space. We're on the same side! We all want the wildlife, whether you want to harvest them for a crop, or whether you want to love them for a photograph, you still want them on the landscape.

Like Monique, at least three others discussed big industry (i.e., mining, logging, oil) and its impact on the environment, and ultimately, wolf habitat. For instance, when asked about the main barriers facing wild wolves, Monty stated: “[T]hat's people. Everything from direct or indirect habitat destruction through resources. And one of the worst, is now some of the big strip mining programs, oil [industry] up in Canada."

Habitat fragmentation and how wolves are unable to disperse due to large urban/population areas or major highways cutting through their territory, and how this sometimes has an effect on their genetic health, were also issues of concern. These educators also discussed wolves' inability to adjust to living in urban and semi-urban landscapes thus the importance of having large protected wild spaces for predators like wolves. Rachel was one of six educators who explained that wolves could not return to their former range in the United States due to urbanization: "I personally think they're never going to be able to reclaim their original habitat. I mean humans are just too much, there's too many of us. So if anyone thinks they should get back to where they originally 
were, I think, is kind of kidding themselves." Monique asserted that when wolves were reintroduced to areas, biologists needed to make sure the habitats were large enough to sustain a population: 'Because some of the release sites that I've seen, boy, you don't put wolves in marginal habitats with marginal prey dynamics and prey numbers and expect them not to turn to some other food [source]."

\section{Human-Caused Mortality}

When it came to obstacles facing wild wolves, six educators discussed the direct and immediate impact of human-caused mortality through illegal and legal killing or roadkills. For example, Jeremy reported that in Idaho, over $90 \%$ of wolf mortality was due to humans. Other educators talked about illegal killing or poaching, especially in areas of reintroduction. As well, I heard from the two Colorado educators that part of why Grey wolves have had trouble dispersing from Wyoming to Colorado is that they have to cross a large highway (the I-70) to do so and likely get killed when attempting to do so. Rachel explained:

Wolves have crossed over into Colorado, but their biggest issue is, you've got I$70 \ldots$ and usually they're coming down from Wyoming, and when they come across the highway, we think, at least our hypothesis is that they see the highway as a game trail. So "Oh, if I follow this I'm going to run into some elk" and then unfortunately it's not elk that they run into... So that's been the biggest issue is that they can't seem to get past I-70.

\section{Barriers to Conservation: Discussion Points}

All of the barriers facing wild wolf conservation named by the educators (negative attitudes, misinformation, politics, habitat, human-caused mortality) have been discussed in the literature. Social scientists have examined human attitudes towards wolves, wolf reintroduction, and management fairly extensively (for examples, see Bath, 2009; Karlsson \& Sjostrom, 2007; Naughton-Treves et al., 2003; Skogen \& Thrane, 2008; 
Williams et al., 2002). Further, both wolf biologists (Boitani, 2003; Chapron, et al. 2014; Musiani \& Paquet, 2004; Eisenberg, 2014) and social scientists (Bath, 2009; Bruskotter et al., 2007; Kellert et al., 1996) maintain that people's attitudes, and consequently their political support for wolf conservation, will have a significant affect on whether wolves will continue to survive and/or flourish on the landscape. Issues related to wolves having adequate and appropriate habitat have also been discussed (Boitini, 2003; Eisenberg, 2014), although in North America usually this means wilderness or wilder areas whereas in modern Europe, wolves have managed to live in close proximity to human populations fairly successfully (Chapron et al., 2014). Finally, there is some research to support the educators who felt that, at least in the northern Rockies region of the United States, humans contribute significantly to wolf mortality, however, I did not find any statistics to suggest it was as high as 90\% (Creel \& Rotella, 2010; Murray et al., 2010). Especially estimates that include illegal killing of wild wolves are obviously difficult for biologists to determine.

The fact that the educators cited the same barriers to wolf conservation that have been researched and discussed in the literature demonstrates their extensive knowledge of the situation for wolves. Clearly, their concerns reach beyond the wolves at their various programs/centres to encompass wild wolves, and to some degree provides another substantiation that conservation is indeed one of their primary motivators as educators (as discussed in chapter six). While I cannot verify that they were actually sharing these messages with visitors to their programs since I did not observe them in practice, I can confirm that these educators were clearly knowledgeable about and engaging in issues around wolf conservation. 


\section{The Ethics of Conservation and Management}

The educators' concern for wild wolves also extended to how they felt about the present management and conservation of wild wolves in their region or country. While discussing conservation and management, some of my participants brought up ethical concerns with regards to wolf management strategies, often arising out of their own experiences working with wolves. I found that the ethical considerations raised were particularly salient and insightful, in some cases speaking directly to the literature on the wolf debate, and in other cases offering unique perspectives worthy of consideration. The most common topics that arose in discussions of the ethics of conservation and management included: that humans should adjust to share the landscape with wolves, that management should take into account wolves' social nature, the place of science in decision-making, and the inhumane and/or unethical nature of certain management techniques.

\section{Humans Should Adjust to Living with Wolves}

Seven educators were clear that that they felt that humans need to change their behaviours in order to share the landscape with wolves. Some stated this as a philosophical principle (e.g., wolves have a right to be here therefore humans should adapt to share space), while others took a more practical stance (e.g., ranchers need to adopt measures to deter wolves). For instance, Gwen discussed how in areas where predators and people live in close proximity, “[bears, cougars, wolves] don't have the same ability to adjust their behaviour that people do. And so the change, if we want animals in more places, the change is going to have to come, I think, from us... and not expect the animals to be able to change as much." Monique too believed that humans 
should adjust to make space for other species, saying: "You know, we're all co-existing on this planet. And I'm not a believer of egocentric management. I mean, I don't believe as a member of the human race, that's not a philosophy that I support. And whenever I can professionally help recognize that there are opportunities for other species, I will do that."

Some educators focused on practical adaptations that ranchers, farmers, and hunters may have to make with wolves on the landscape. Jeremy, for example, talked about how, in Idaho, wolves have changed the behaviour of elk, making them harder to hunt. While once elk browsed comfortably out in the open, the return of wolves means they are now more likely to seek cover. He continued, "So, human hunters almost have to evolve now and become more like traditional hunting. You've got to go find your elk to take it."

Some educators also talked at length about how ranchers may have to change their practices. I heard about techniques to deter wolves, such as a return to having cowboys riding a farm or ranch range more frequently (i.e., range-riding), installing radio-activated guard boxes (RAG boxes) ${ }^{7}$ and the use of "fladry." 8 For example, Shelley told me about how she encouraged a local rancher to put deterrents in place: "I told him, 'Start riding your range, and seeing what's out there and what's chasing your livestock.' I said, 'Are you spending any time out there?' And he's, 'Well, you don't need to do that.' I said, 'Well, in this day and age you do need to do that. You need to protect your investment."'

\footnotetext{
${ }^{7}$ RAG boxes are wolf deterrent devices usually placed around the perimeter of livestock corrals and ranges. They have strobe lights and "annoying" sound recordings that are triggered by the frequencies sent off by collared wolves who come in close proximity.

${ }^{8}$ Fladry are strips of (usually) red fabric that are attached to the perimeter of a livestock area. They wave and flap in the wind and are intended to scare off wolves.
} 
Monique also discussed how ranchers and farmers need to make adjustments not only for the sake of their livestock, but also for the sake of wild animals. "And agriculturalists need to be, I think, accountable for their animal husbandry practices. And that it shouldn't always be the wildlife that gets killed when there's something a human can do to improve practice."

\section{Management Should Account for Wolves' Social Nature}

Another ethical matter five educators raised was the idea that management of wild wolves should take into account the social nature of wolves. Often the educators discussed this idea in relation to their personal experiences with captive wolves. For example, Monique explained how experiences with captive wolves caused her to question typical management practices: “[I]n captivity I see the individual because they're relaxed enough to display the individual personality. And when I see the individual, I do believe that there are impacts that people are not registering when we only manage a population."

Most commonly, these educators discussed the potential implications of not knowing an individual wolf's role in a pack and consequently disrupting or destroying a pack when wolves are killed as part of a conservation management strategy. For example, if the best hunters or pack leaders are killed, the young wolves might starve, be less cautious around people, or not have the knowledge to hunt wild prey and turn to livestock for food. Case in point, when asked what he wished the general public understood about wolves, Matthew answered:

I would like people to understand about wolves that they are social animals. They live in social groups, that those bonds and connections are vital to them... When we're looking at management of them, we have to take into account that social aspect of things. And they need to be used as part of decision-making processes. I think that they're often overlooked... There's some evidence, not confirmed, but some evidence that disruption of social orders of wolves, like through a hunting 
and a trapping season, could actually..., like if the alpha pair are killed, that pack might actually be more likely to attack livestock?... Where because the most experienced members of the pack, that are most skilled in hunting elk or deer and things like that, have been eliminated.

The exact same concern was expressed by three other educators.

\section{Science-Based Decision-Making}

Seven educators discussed the interplay of scientific knowledge of wolves and the conservation and management of wild wolves. Clearly, some of them felt that management decisions around wolves, such as how many wolves to hunt in a certain state or province, should be based on the available science rather than popular opinion or the political climate. For instance, Erik stated, "Unfortunately, so many decisions today on wolves are based on emotional, are based on politics, you know, so little on science. So many of the decisions are not based on science. It's so frustrating." Similarly, Monique shared her frustration that some management and decision-making teams in certain states do not even have wildlife biologists involved. She said:

You hope to rely on your resource agencies to provide you sound science, and to be able to disseminate that sound science to the public... and biology's gone out the window, and that's the hard part. I've never seen a situation where wildlife management..., where wildlife managers are not even at the table. You know, it's politicians and it's special interest groups making the decisions.

Likewise, Jeremy explained how decision-makers in Idaho, for political reasons, had ignored and even changed research reports estimating the present wolf population, so much so that the state biologists became frustrated enough to quit their jobs. He explained, "We've lost a lot of good biologists in the state because of things like that happening. So, that's our big beef, is wolves are not being managed biologically in this state. They're being managed politically and that's incredibly frustrating." 


\section{Inhumane and Unethical Treatment}

Five educators expressed concerns about certain management techniques being inhumane and unethical. Examples of problematic techniques identified included: allowing hunters to shoot lactating females, destroying an entire wolf pack or multiple packs when it is one wolf who is predating on livestock, sterilizing and re-releasing wolves, government killing of animals in secret, a wolf-hunting contest with awards for the smallest/youngest wolf, and indiscriminate killing of wildlife with cyanide tablets.

For example, Jeremy detailed:

[R]ight now it's completely legal in Idaho to shoot a pregnant female...Yeah, and animal humane groups are, like, what? When do we do that with any other species? We can actually shoot a lactating female right now that has puppies, or obviously if she's lactating she's got puppies going on, and what's happening to those puppies? Of course they're starving to death.

Shelley reported that "last year, there was a competition going on in Fort St. James where they got prizes for the nicest pelt, and they got prizes, and they got paid a booby prize for the smallest wolf they could catch. How humane is that? That still goes on in 2014!" Spencer, too, expressed frustration: “And, you know, we're still eradicating animals. The organization Wildlife Services, [a] federal program paid by our tax dollars, you know, they kill four million animals every year, a lot in secret."

Along with concerns about specific inhumane and unethical management techniques, three educators questioned the ethics of humans even trying to control wildlife in the first place. For example, Christina discussed the dwindling population of wolves on Isle Royale and questioned whether humans should lend a hand to help. I asked her what she would do if given the power to choose and she replied, "You know, the wolf-lover in me, I would love to help them. But at the same point in time, if we're 
trying to be as hands off and let it happen naturally, you know, you would let the population die off. The biggest question though is: were they naturally put in this position? Or did it have to do with people?"

At the root of concerns like what Christina expressed is questioning what our human responsibility towards wild animals ultimately might be. While the above educators felt that some management techniques were unethical and inhumane, eight nonetheless also seemed to believe that when it came to wolf management, however they personally felt about management practices, collaboration and compromise with those who hold opposing views might be necessary. For example, they argued that those who want wolves to remain on the landscape may have to accept that wolves would need to be "managed" to some degree, including being hunted, trapped, or destroyed when they predate on livestock. Jeremy stated:

Promoting that type of tolerance and also accepting the fact that some wolves are going to die. We're not a pro, radical wolf kind of organization that says no wolf needs to die. Sometimes there's going to be wolves that are just bad seeds, that are just doing things that they shouldn't be doing and they're killing dogs or they're non-stop killing livestock regardless of how much we try to prevent it. When those kinds of things happen, some wolves need to be sacrificed for the betterment of the rest of the population. So, we understand that.

Michelle, too, talked about how, in Oregon, ranchers are required to use non-lethal wolf deterrents but if anti predation techniques have been attempted and have failed, she stated,

If the wolves do come back, and they don't respond to the hazing, then they can be lethally removed, which, I mean in my opinion, you give the wolves a chance, if they don't respond to it, then you're taking the next logical step. But unfortunately, everywhere else is just like, "Ah, shoot them if you see them." It's so hard. 
Given wolf conservation and management is so controversial, Erik explained how the heightened tensions can lead to a situation that no matter what decisions are made, there are winners and losers. He argued, “That's not going to solve anything, because then there's just going to be winners and losers. And the losers, whoever it turns out to be, are going to be resentful, and it's just going to cause problems." He talked about how the Centre where he works, Wolf Haven, is involved in the Wolf Advisory Group (WAG) in Washington state which is comprised of representatives from various interest groups (i.e., ranchers, hunters, conservationists) and they collaborate to make management recommendations. Erik said, "Really it's just sitting down to the table and figuring things out, and coming up with something that everybody can agree to, that's the only way that conservation is going to work long-term."

\section{The Ethics of Wolf Management: Discussion Points}

Many of the educators had concerns regarding the ethical treatment and management of wild wolves. Wolf biologists, ethologists, and ethicists have raised some of the same concerns (Eisenberg, 2014; Fox \& Bekoff, 2009; Jickling \& Paquet, 2005; Lynn, 2007). For example, Fox and Bekoff (2009) question the ethics of much wild wolf management in light of both wolves' social nature and their lives as individuals. They write: "In conservation biology, the interests and rights of individuals are sometimes traded off against perceived benefits that accrue to higher levels of organization: populations, species, and ecosystems" (p. 127). Fox and Bekoff point to research on animal cognition and emotion that demonstrates animal-others have their own interests and points of view. They argue that this needs to be taken into account by those making decisions about wolves, stating that doing so: 
...forces us to consider their needs and interest as individuals, as families, and as members of a community. Because the wolf is a species with complex social structures and tight family bonds, we must consider the ethical implications of our actions when we disrupt family packs through management and control programs. (p. 125)

Musiani and Paquet (2004), in their overview of wolf protection and management in Canada and the United States, suggest that the fact that some sectors of the public are becoming increasingly aware and sensitive to certain types of wolf management has only added to the contentiousness of the issue. They state, "Such controversy has the potential to exacerbate already entrenched and inflexible positions in the confrontation between wolf preservationists and anti-wolf advocates" (p. 54). What this may mean for the wolves who are being protected and managed is difficult to tell at this point.

The role of scientific knowledge in wolf management also plays into the mix here. As noted, some of the educators are concerned that scientific knowledge is being either discounted when decisions are made or overridden by politics and/or popular opinion. There are some biologists and social scientists who have raised similar concerns to these educators, noting that in some instances, political pressure and/or popular opinion hold more weight than scientific research when it comes to decision-making around wild wolves (Bruskotter, Vucetich, Enzler, Treves, \& Nelson, 2014; Eisenberg, 2014). It is not surprising that these educators value scientific knowledge given most of them had academic backgrounds in science and/or natural resources, their centres tended to emphasize scientific knowledge, and much of what they wanted to share with visitors was scientific facts about wolves, as discussed in chapters five and six. It is no wonder, then, that their ideas about wolves and issues such as management were strongly grounded in a science-based framework. 
An alternative view on this issue is offered by Lynn (2010) who believes scientific perspectives are important but nonetheless challenges the privileging of science discourse in the study of wolves and human-wolf relations over other important discourses such as social and ethical ones, which have often been ignored. He believes a greater acknowledgement of the social and especially ethical issues related to wolf recovery is needed and that these should play as important a role in policy and management decision making as biology or ecology. He notes, however, that any approach is incapable of neutrality. He writes, "whether our theories are hunches, testable propositions, or expansive worldviews, they are always fallible, contestable, and in the making" (p. 85).

Finally, collaboration and compromise as discussed by the educators above, including involving various interest groups in decision-making processes about wolves, has been attempted with some amount of success (Bath, 2009; Bisi et al., 2007; Todd, 2002). Managers and decision-makers in some areas share Erik's view that problems are compounded when there are winners and losers, and this in turn, could ultimately impact the success of wolf conservation efforts.

\section{Conclusion}

At the heart of this discussion about conservation and management decisionmaking is the ethical question of who should get to choose what happens to wolves? Should politicians get to make decisions about the survival of wolves and on what grounds? Should decisions be based purely on scientific research and what is predicted to be good for the ecosystem? Should the focus be on the health of wolves as a species or should the lives of individual wolves matter too? Should the people in the region where 
the wolves reside, the people who are actually living with wolves, have a greater say in their management, or should everyone across the state, province, or country have the right to weigh in? When, if ever, is wolf killing justified, and how? Ultimately, do wolves have a right to exist on the landscape and live out their lives even if it means humans will have to make adjustments to accommodate them?

Clearly, then, there is much more involved in wolf conservation and management than scientific knowledge about their importance to ecosystems or tallies of the small numbers of livestock they kill. Whether or not decision-makers acknowledge it, ethical considerations around our relationships with, and responsibility to, other individual animals and species lie at the foundation. As Bekoff (2007) writes, how we choose to exercise the power we have over the lives of other animals speaks to who we are as humans: "The power we potentially wield to do anything we want to do to animals and nature as a whole is inextricably tied with responsibilities to be ethical human beings" ( $\mathrm{p}$. 918). It was clear to me that many of the educators I interviewed were well aware of the "power we potentially wield" over wolves and their survival.

Something that stood out to me during the interviews, and was confirmed by the emergent overarching theme, "Engaging in the Controversy of Wolf Education," was that to some degree, for all of the participants, being a wolf educator meant dealing with a controversial conservation subject in an educational context. This was true especially for those who worked at centres in wolf territory. But even for those who did not, the fact that aspects of wolf survival and management are contentious nationally in both Canada and the United States meant that all of the educators had to deal with the controversy on some level. Given the findings, I propose that in order to negotiate this controversy in 
their work, most of them were compelled to seriously contemplate their own ethical positions related to both education and wolf conservation and management. I would argue this is more so than they might have had they been educating about a less contentious subject.

As this chapter has shown, the experience of being a wolf educator involves contemplating some of these ethical questions around wolf conservation and management, and more broadly our responsibilities to, and relations with, other animals. Many of the educators had developed fairly extensive knowledge about wolf conservation issues, demonstrated evidence of having explored and formed ethical positions on a number of matters, and honed their ideas about how to navigate these controversial issues in a professional educational environment. I suggest that all of these were influenced by their care for and experiences with specific wolves at their programs as well as the contexts in which they were educating, which brings me to my concluding chapter. 


\section{CHAPTER 8}

\section{CONCLUSION}

To conclude this dissertation, I return now to my research question and subquestions. Van Manen (2014), in discussing the evaluation of phenomenologically based studies, asks whether the study has offered "us the possibility of deeper and original insight, and perhaps, an intuitive or inspirited grasp of the ethics and ethos of life commitments and practices?" (p. 356). With that in mind, I ask myself if and how this study of the experiences of wolf educators has offered insights into "the ethics and ethos" of what it means to educate about, and work with wolves? As a reminder, I embarked on this research asking the following question: What are the experiences of educators who work at programs that feature captive or wild wolves; in particular, what do these educators learn through both working with and teaching about wolves? I had also hoped to answer the following sub-questions:

- What are the key messages that educators hope to offer participants in their programs?

- How has working with and teaching about wolves had an impact on their personal attitudes and beliefs about wolves?

- What do educators learn through working with wolves at wolf programs, both about wolves, and from the wolves themselves?

- What ethical issues, if any, are raised for them in using wolves in education programs?

- Do educators think education about wolves makes a difference when it comes to conservation? Why or why not? If yes, how so? 


\section{The Experience of Being a Wolf Educator}

As noted, the purpose of this research was to investigate the experiences of educators who work at centres that feature captive or wild wolves. While each educator obviously had their own unique experiences with wolves and wolf education, when it came to being a wolf educator, certain motifs emerged that help elucidate the experience. These included: the significance of political, regional, and cultural contexts; the key role that scientific knowledge and learning has for many wolf educators; the importance of ethics when it comes to teaching about and working with wolves; the impact of direct experiences with wolves on how educators understand and feel about wolves; and finally, their engagement with wolf conservation issues.

\section{Context Matters}

The experience of being a wolf educator has its unique aspects, reflecting the educators' personal contexts (e.g., their backgrounds, beliefs, and values) and their professional contexts (e.g., the missions and educational philosophies of the centre and the region where the centre/program is located). It is difficult, if not impossible, to draw a clear line between the influence of cultural, political, and regional contexts, but it is clear that all three shaped the educators' experiences. For example, some educators' choices to share primarily scientific knowledge about wolves rather than advocacy messages were likely influenced by the regional political climate in which the centre or program was located. Case in point, the seven educators who discussed "sharing only science" were all located in areas where wolves reside and the controversy is more heightened and politically charged. Politics, too, shape how wild wolves are protected in 
various regions, which in turn, had an impact on the experience of those educators who cared about and were invested in the protection and conservation of wild wolves.

Culture also had a considerable impact on the experiences of the educators. For example, at least 11 of them argued that culture was key in understanding why many people in North America, including visitors to their centres and programs, continue to feel fearful of and/or negatively towards wolves. As the educators argued, these reflect deep cultural roots and have a long history. It is no surprise, then, that many of the educators devoted some of their efforts to busting myths about wolves and attempting to offer counterstories to the fables, myths, and movies that often portray the wolf unfairly or unrealistically.

The importance of context, whether regional, political, and/or cultural, in shaping experience is not new. Writing about experiential environmental education, Russell (1999) suggests that people are likely drawn to certain experiences based on what they already value, and in the case of the educators I interviewed, their love of nature, animals, and/or wolves specifically drew many of them to be wolf educators in the first place. While the fact that their experiences were shaped by both their own contexts, and the contexts in which they worked, may not be new or surprising, it is worth mentioning because it reinforces the findings of other researchers who have discussed the importance of context (Kollmuss \& Agyeman, 2002; Liddicoat \& Krasny, 2013; Russell, 1999; Stevenson, Dillon, Wals, \& Brody, 2013). For example, Stevenson et al. (2013) write: “Approaches to environmental learning processes now recognize that worldviews and belief systems shape individuals' understanding and interpretation of environmental issues and mediate their environmental behaviours" (p. 513). Others have made similar 
points about the importance of context in understanding peoples' attitudes and beliefs about wolves (Boitani, 1995; Nei, 2002; Shelley et al., 2014; Skogen \& Thrane, 2008).

\section{Scientific Lens}

Having interviewed 17 educators, observed at two sites, and conducted a content analysis of centre and program websites, I can confidently say that the experience of being a wolf educator involves understanding, interpreting, and teaching about wolves and wolf issues through the lens of western science. The following points are demonstrative: most of the educators had educational backgrounds in science or natural resources; knowing and sharing the science of wolves was a priority for most of them, so much so that some of them felt that wolf education should be mostly limited to sharing science-based information; the content of two talks I observed at one centre almost exclusively focused on science or ecology-based information about wolves; at least six of the educators had been involved in scientific research on wolves; and some felt that decisions about wild wolves should be based in scientific research rather than on public opinion or politics.

It is important to make clear, however, that while understanding and teaching about wolves through a science-based framework seemed commonplace, the educators did not entirely limit themselves to this approach. For example, while some educators talked extensively about wolf ecology during the interviews, the same educators also discussed the importance of creating emotionally meaningful experiences or described how their own experiences interacting with actual wolves offered different learning and insights from what they had gained during their science-based education. Thus while the 
experience of being a wolf educator commonly meant understanding and educating about wolves through a scientific lens, this lens was not used exclusively.

Their understanding of western scientific concepts affected the experiences of the wolf educators, and influenced their ideas about wolves and wolf issues, as well as their approaches to educating. However, many scientists involved in conservation agree that decisions cannot rely only on an understanding of scientific concepts, but involve often difficult and complex ethical and moral aspects as well (Bekoff, 2013a). Likewise, in science education generally, increasingly there has been a move to focus not only on science concepts but also include discussion of the social, economic, and political aspects of controversial issues, including conservation (Oulton, Dillon \& Grace, 2004; Russell \& Hodson, 2002). Oulton et al. (2004) contend that this needs to be taken further, arguing that science education should include not just the investigation of controversial topics, but help students understand the nature of controversy itself, including how all people approach issues from different worldviews, what the limitations of science are, and the influence of politics and power on science. Such an approach is foundational to STSE (Science-Technology-Society-Environment) education, a field that is now over 40 years old (Pedretti \& Nazir, 2011) yet still remains somewhat on the margins even though, as Hodson (2010) writes, such an approach is vitally needed to meet "the demands, issues, and problems of contemporary life. A much more politicized approach [to science education] is advocated, with major emphasis on social critique, values clarification, and sociopolitical action” (p. 197). On this note, Wals, Brody, Dillon, and Stevenson (2014) believe that environmental education, with its attention to understanding the contexts and processes whereby people develop critical, ethical, and creative thinking and action when 
it comes to the environment, has much to offer science education, and that the two together could be especially effective, especially in the face complex environmental issues. They write:

So, although SE [science education] may have evolved separately from EE [environmental education], recent research and developments in both EE and SE converge toward generating an interdisciplinary and contextual approach to integrating research in science, education, and the environment. (p. 584)

Unfortunately, it may take some time to see such movement in informal science education learning environments like those within which the wolf educators work. As Lloro-Bidart and Russell (2016) have demonstrated, informal science and environmental education that takes place in zoos, aquariums, and in parks and protected areas tends to be conservative in terms of both content and pedagogy. They find this depoliticized approach to informal science and environmental education disheartening and argue that it is simply not up to tackling the thorny issues of environmental destruction and exploitative human/animal relationships. That being said, some of the educators in this study did touch on some of these issues, and all were very much aware of the political contexts within which they were working and the ethical dimensions of human relationships to wolves were important to them, as I will discuss in the next section,

\section{Ethical Engagement}

Ethics mattered very much to the educators in this study, as demonstrated in their discussions of: the ethical dimensions of education, including with captive or wild wolves; how to care for wolves; wolf conservation and management decision-making; and the broader issue of how humans treat wild animals in general. At the fore of the ethical concerns discussed was the comfort and happiness of the wolves involved in their educational work. For example, some felt it was more ethical to have the wolves as 
socialized and comfortable around humans as possible so that they were not stressed and so that visitors would get a more accurate sense of their behaviours, while others discussed how having the wolves remain unsocialized showed respect for their inherent wildness and allowed visitors to witness wolves' natural fear of people. A number of educators argued that, ethically, the care and treatment of the wolves in their centres should be more important than the enjoyment or entertainment of the visitors, and some believed that wolves in their centre should never come from breeders but should only ever be rescued animals. For me, the various ethical stances of these educators must be taken seriously; their positions hold weight given their insights were grounded in time spent caring for, coming to understand, and building relations with actual wolves. Indeed, I propose their ethical insights are especially important because they have been formed through actual relationships.

Jickling and Paquet (2005) argue that ethical positions are often born out of care, namely out of what and who we care about: "[E]thics is largely about care, what entities warrant our care and consideration, and how we should behave toward those entities that demand this care" (p. 129). The idea of an "ethic of care", then, may be applicable here. Some environmental education scholars have explored the implications of an ethic of care from various vantage points (e.g., Martin, 2007; Mortari, 2004; Russell \& Bell, 1996). For example, Martin (2007) suggests care is important in environmental education, but argues it is difficult to foster caring about environmental issues by providing students factual information alone. Similarly, building on ideas from ecofeminist scholarship, Russell and Bell believe that "logic and abstractions" (p. 175) should not "be privileged at the expense of other ways of knowing" (p. 175). Martin (2007) discusses the 
importance of students coming to see their relatedness and reciprocity with nature as well as making room for both conceptual and emotional learning:

[S]tudents need to understand their relatedness to the environment as a subjective relationship, individual to individual. Caring for nature is also a challenge to any form of education that interprets nature as an external generic object, or set of objects. Rather, environmental caring education must seek to understand both rationally and emotionally the places, entities and nonhuman individuals with whom students develop specific personal lived relationships. (p. 62)

Russell and Bell (1996) agree, but push these ideas further, arguing for inclusion of a more critical dimension, advocating for a "politicized ethic of care" that can better take into account, and build on, the various contexts within which environmental education occurs (see also Lloro-Bidart \& Russell, 2016).

The educators I interviewed very often seemed to have developed their own ethical positions on wolves through their experiences and relationships with actual wolves and there were conceptual, emotional, and political dimensions to this learning. While many of the educators shied away from the political dimensions in their work, they did want visitors to learn the "truth" about wolves and to have similar embodied experiences whether through observing, howling with, or interacting with wolves. While it is worth noting that most of the educators learned about, and developed relationships with, wolves in the context of captive settings, their own direct experiences with wolves was clearly important, which brings me to the next sub-theme.

\section{Learning About Wolves Through Experience}

Being a wolf educator involved coming to understand wolves through knowing actual wolves rather than learning about them in the abstract. Through experience, many of the educators came to believe that wolves have agency, are capable of complex social interrelations and communication, and that each wolf is an individual with her or his own 
unique personality. The 11 participants who interacted directly with wolves through care and training formed particular interspecies relationships that were unique depending on the wolf and person in question, and formed bonds that were grounded in nurturing and sometimes in trust. For these educators, the experience of knowing and caring for wolves informed their insights into the ethical dimensions of how wild wolves are treated and managed.

As discussed in the findings and discussion sections, some of their insights about wolves garnered through experience are resonant with the literature. Increasingly over the last few decades, the idea that other animals are unique individual subjects with personalities, agency, and rich social emotional lives is being discussed, researched, and accepted (e.g., Bekoff \& Pierce, 2009; Fawcett, 2002, 2013; Irvine, 2004; Kuhl, 2011a; Sanders, 1999). We can draw both pedagogical and ethical conclusions from this finding.

First, direct experiences, that is, having actual relations with other animals in gaining an understanding about their abilities, capabilities, and complexity is pedagogically important. Based on her research in which some children had a short direct encounter with a common wild animal (i.e., bat, frog, or raccoon), Fawcett (2002) drew a similar conclusion, "Thus, one of my essential findings is about the importance of direct experience for positive human and other animal relationships, and the implications of this for biological conservation and environmental education" (p. 131). Of course, it is logistically unrealistic, not to mention rife with ethical conundrums, to think we can have every school child meet a wolf or any number of wild animals for that matter. However, there are all kinds of opportunities for both children and adults that could be facilitated through environmental or humane education in formal and informal settings that involve 
direct encounters with other animals that may help people gain some of the same insights discussed by the educators I interviewed (e.g., other animals' capabilities, personalities, and agency).

Second, if experiences and relations with other animals can be rich and complex and lead people to seeing them as having agency as happened with the educators in this study, it raises question about how we humans treat them. As discussed by some of the educators, these insights could help inform how we manage wild wolves on the landscape, an idea that resonates with researchers pondering the ethics of human-wolf relations (e.g., Fox \& Bekoff, 2009; Lynn, 2007). For example, perhaps there are better ways to respond to wolves who are predating on livestock than killing members or only a portion of a pack, especially considering we know how integral pack social structure is to the survival and wellbeing of individual wolves. Further, given wolves' complex social and emotional lives, we should call into question certain management strategies that very likely cause wolves great pain and distress (Fox \& Bekoff, 2009; Jickling, 2005a; Lynn, 2007).

Finally, some environmental and humane educators suggest that the learning that emerges from direct experiences with particular animals may enable the development of a broader ethic regarding how we treat other animals more generally, for example, in factory farming, the food industry, and research (e.g., Fawcett, 2013; Lloro-Bidart \& Russell, 2016; MacPherson, 2011; Pedersen, 2011; Weil, 2004). Bekoff and Pierce (2009) draw a similar conclusion based on their expertise in human/animal relations and animal ethology: "In drawing a picture of animals as beings with rich cognitive, emotional, and social lives, wild justice invites a serious reconsideration of the uses to 
which we put animals in research, education, and for clothes and food, among other things" (p. 137).

\section{Wolf Education for Wolf Conservation}

For the participants in this study, the experience of being a wolf educator meant engaging with conservation issues whether it was developing their own understanding of wolf conservation science, teaching people about wolf conservation, or weighing in on issues of wild wolf conservation and management. I asked all of the educators whether they thought education can make a difference when it comes to wolf conservation, and all of them felt that either it can and/or that it already has. In fact, some of them felt it was the only feasible avenue for cultivating humans' willingness and/or tolerance to share a world with wolves. Wolf education, they explained, can contribute to wolf conservation and survival through: dispelling myths, thereby helping people overcome misconceptions about, and fear of the wolf; creating meaningful experiences for people, ones that help them feel a connection with wolves and nature and that might instill care for and action on behalf of wolf survival; helping people understand the similarities between wolves and humans or companion dogs, perhaps in order to evoke cross-species empathy; changing attitudes, especially by educating younger generations; and finally, by engaging in broader outreach such as working directly with ranchers and farmers to implement coexistence strategies.

Educators' ideas about pedagogy were also often focused on and intertwined with their ideas about conservation. This was particularly clear in the assertion that they needed to meet the visitor wherever they were at and to be open to listening to their ideas and viewpoints about wolves and wolf issues. For instance, when discussing the key 
messages they were hoping to share with visitors, most commonly, the educators discussed the importance of wild wolf conservation and/or the message that wolves do not make good pets. How they felt these were best achieved through education varied, with some suggesting that sharing the science of wolves and allowing visitors to come to their own decisions about whether or not to support wolf recovery and others arguing that it was acceptable to encourage visitors to advocate on behalf of wolves. As discussed earlier in the dissertation, when faced with teaching about controversial issues, environmental educators also sometimes struggle to walk the fine line between encouraging advocacy and indoctrination (e.g., Jickling, 2005b).

At least some of the educators I interviewed had obviously wrestled with how best to offer visitors information about wolves and wolf conservation without impinging on their freedom to come to their own decisions based on their personal values and beliefs. A growing number of education scholars have discussed how one might approach advocacy related to other animals (e.g., Humes, 2008; Jickling, 2005b; Pedersen, 2010; Taylor, 2009). For one, Jickling (2005b) reminds us that it is impossible for education to be completely objective and that educators inevitably come to their roles with their own set of beliefs and values and things they care about and may want to advocate for. Retreating from this challenge is not an option according to Jickling since he argues that when educators choose not to engage with "controversial issues and radical questions" (p. 110) it sends students the implicit message that doing so is not important and implies that the status quo is acceptable. He believes that despite the inevitable messiness, educators should help learners engage with complex and contentious issues, develop critical thinking skills, and be encouraged to be active citizens. 


\section{Final Thoughts}

As I come to the end of this dissertation, I will briefly discuss future scholarly research that I suggest would be useful to pursue, practical considerations that might be worth pondering by those involved in wolf education, and finally, the role of education in wolf conservation.

\section{Scholarly Implications}

While this research offers a valuable window into the experiences of 17 educators who work at 15 programs, especially with regards to their ideas about wolf education and their personal beliefs about, and experiences with wolves, there are still many gaps in the research when it comes to wolf education. For one, a more complex and arguably more accurate understanding of wolf educators' experiences could be obtained through research that includes more observation, including of educators teaching as well as interacting with wolves. This might also better enable bringing the wolves' experiences into the equation. If the goal of wolf education is to improve human-wolf relations and ultimately the lives of wolves, then research about wolf education needs to seriously consider the experiences and perspectives of the wolves involved. Within the social sciences there has been increased attention in research to consider the perspectives and experiences of people who have been historically marginalized, but it remains uncommon to find researchers who extend this to consider the perspectives and experiences of other animals (Fawcett, 2013). I assert that we need more research that just does that. Interested researchers could look to the fields of humane education, critical animal studies, and critical environmental education for examples of researchers who are engaging in this work (see, for example, Fawcett, 2013 and Oakley et al., 2010). 
On a different note, research that investigates the experiences of the people who visit wolf education centres and programs would be useful in understanding how participants are responding to what is being offered. While my research offers good insight into wolf educators' ideas and intentions, we do not know what participants to these centres are actually learning, intended or otherwise. In particular, I wonder if these experiences have any long-term effect on what participants think or feel about wolves, their ideas and beliefs about other animals more generally, or their willingness to advocate for wolf survival and conservation?

Lastly, while there has been a fair amount of social science research looking at people's attitudes towards wolves and wolf reintroduction, and recommendations that education is one possible avenue for improving attitudes and relations (e.g., Anderson \& Ozolins, 2004; Ericsson \& Heberlein, 2003; Frits et al., 2003), research into whether wolf education actually has any positive impact on human-wolf relations has been scarce. I believe that those involved in wolf conservation research including scientists, social scientists, ethicists, and educational researchers could collaborate to develop a more wellrounded and interdisciplinary approach to wolf recovery and survival that is grounded in research that considers not only the biological and ecological dimensions, but also the cultural, social, and ethical ones.

\section{Practical Implications}

While I certainly sympathize with those educators who were nervous about taking an advocacy position and impinging on the values and beliefs of visitors to their programs, my research leads me to raise the question of whether informal science education should always be so depoliticized and conservative pedagogically and content- 
wise. Taking inspiration from critical environmental education and humane education, it seems to me that there are ways that wolf education could encourage "being for" wolves or conservation more generally while still respecting the values and beliefs of visitors and avoiding indoctrination. For one, educators might acknowledge to participants that it is impossible for anyone, themselves included, to be "unbiased" and that there are a range of positions on the wolf question and many ways to approach the topic, each with its own set of presumptions. In doing so, they might be better able to share their own positions, how and why they came to their positions, and name the assumptions underlying them; doing so would not only help visitors see that the educators' positions are thoughtfully considered and grounded in a particular context, but also allow them to critically consider their own attitudes and beliefs.

Another way educators might help complexify people's views about wolves or offer them new perspectives is through sharing their own personal stories. I found the personal stories and anecdotes the educators shared with me about working with wolves to be powerful and engaging, so I would encourage them (if they do not do so already) to share these same stories with visitors to their programs. Just because these experiences are specific to the people and the wolves in question does not make them any less worthy of consideration. In fact, they illustrated for me that wolves have all kinds of amazing abilities and can be individuals with personalities, interpersonal relations (with each other and with humans), and agency. Further, another insight that I believe wolf educators can offer both visitors to their programs as well as the wider wolf conservation community is their ethical understandings based on close observations of and interaction with wolves. This is an important perspective that is often lost when wolves and wolf issues are 
discussed in general abstract terms. As Fox and Bekoff (2009) write, "In conservation biology, the interests and rights of individuals are sometimes traded off against perceived benefits that accrue to higher levels of organization: populations, species, and ecosystems" (p. 127).

\section{Education for Wolf Conservation}

Sadly, the ultimate fate of wild wolves lies in the hands of humans. Like the educators I interviewed, I believe education can play a role and perhaps already has in helping humans to better live alongside wolves. My research leads me to suggest certain pedagogical strategies might be especially important. First, direct experience with other animals can be powerful enough to shape people's ideas and ethics, thus wolf education that has experiential components may be able to play an important role in wolf conservation education.

Second, addressing controversial conservation issues through education necessitates an approach that does not shy away from the messiness but rather directly engages it, helping people understand the nature of the controversy itself. There are so many factors that play into how people feel about wolves that it is naive to think that education based purely on scientific "facts" and concepts will have much impact. I am not suggesting that there is anything wrong with education that includes scientific information about wolves, but rather that wolf education that also offers a critical component and helps people to examine and understand some of the cultural, social, historical, and political elements that play into our past and present human relations with wolves could be especially beneficial. 
Third, just as the experiences of the educators in my study were influenced by their various contexts, I expect so too ought the approaches to finding ways to share a landscape with wolves. Given what I have learned from my research, I find myself agreeing with Boitani (2003) who writes, "there cannot be a single recipe for wolf conservation that can be applied in all ecological and social contexts. Rather, there are several diverse solutions depending on the needs of both humans and wolves at the local level" (p. 340). Educating people who visit wolf centres or participate in programs is clearly an important strategy. So too is the work of those educators who are working with local ranchers and farmers or who are involved in advisory groups where representatives from various interest groups collaborate to advise conservation decision makers; indeed, this seems to me to be a particularly promising track given they are reaching those who may not necessarily be motivated to visit a wolf centre. In all these pedagogical endeavours, however, I do worry that oftentimes the perspectives of the particular wolves involved are missing, which brings me to my final point.

We share our planet with a host of other species and we are in many cases dependent on each other for continued survival. As Bekoff (2013a, 2013b) explains, we cannot ignore our interdependence with the rest of nature indefinitely without facing some pretty dire consequences. He advocates for "compassionate conservation," which is a movement that encourages conservationists to consider not only other animals at the population level, but also ponder and blend this with the welfare and well-being of the individual animals involved. He writes, "if we develop compassion and empathy there is hope for our planet, all of its inhabitants and landscapes" (2013b, p. 385). Simply put, I agree. For us humans to live well in a world with wolves, we need to extend our empathy 
and compassion beyond the human realm to include other species. Based on the results of this study, I assert that humane and environmental education do indeed have the potential to play a role in helping people find ways to share this planet with other animals, including wolves. 


\section{References}

Abram, D. (1996). The spell of the sensuous. New York: Vintage Books.

Anderson, R. E., Hill, B. L. C., Ryon, J., \& Fentress, J. C. (1995). Attitudes and the perception of wolf social interactions: Implications for public information programs. In L. N. Carbyn, S. H. Fritts, \& D. R. Seip (Eds.), Ecology and conservation of wolves in a changing world (pp. 341-350). Edmonton, AB: Canadian Circumpolar Institute.

Andersone, Z., \& Ozolins, J. (2004). Public perception of large carnivores in Latvia. Ursus, 15(2), 181-187.

Angrosino, M. V. (2005). Recontextualizing observation. In N.K. Denzin \& Y. S. Lincoln (Eds.), The Sage handbook of qualitative research ( $3^{\text {rd }}$ ed., pp. 1-32). Thousand Oaks, CA: Sage Publications, Inc.

Antonio, D. (1995). Of wolves and women. In C. J. Adams \& J. Donovan (Eds.), Animals and women: Feminist theoretical explorations (pp. 213-230). Durham, NC: Duke University Press.

Ardoin, N. M., \& Heimlich, J. E. (2013). Views from the field: Conservation educators' and practitioners' perceptions of education as a strategy for achieving conservation outcomes. Journal of Environmental Education, 44(2), 97-115.

Ballantyne, R., Packer, J., Hughes, K., \& Dierking, L. (2007). Conservation learning in wildlife tourism settings: Lessons from research in zoos and aquariums. Environmental Education Research, 13(3), 367-383.

Ballantyne, R., \& Packer, J. (2009). Introducing a fifth pedagogy: Experience-based strategies for facilitating learning in natural environments. Environmental Education Research, 15(2), 243-262.

Bath, A. J. (2009). Working with people to achieve wolf conservation in Europe and North America. In M. Musiani, L. Boitani, \& P. C. Paquet (Eds.), A new era for wolves and people: Wolf recovery, human attitudes, and policy (pp. 173-199). Calgary, AB: University of Calgary Press.

Bekoff, M. (2007). Human anthropogenic effects on animals. In M. Bekoff (Ed.), Encyclopedia of human-animal relationships: A global exploration of our connections with animals (Vol. 3, pp. 909-919). Westport, CT: Greenwood Press.

Bekoff, M. (2010). Introduction: Why animal rights and animal welfare matter. In M. Bekoff (Ed.), Encyclopedia of animal rights and animal welfare (2 ${ }^{\text {nd }}$ ed., Vol. 1 , pp. xxix-xxli). Santa Barbara, CA: Greenwood Press. 
Bekoff, M. (2013a). Who lives, who dies, and why: It shouldn't be all about us. In M. Bekoff (Ed.), Ignoring nature no more: The case for compassionate conservation (pp. xiii-xxvii). Chicago, IL: University of Chicago Press.

Bekoff, M. (2013b). Some closing words: Moving ahead with heart, peace and compassion. In M. Bekoff (Ed.), Ignoring nature no more: The case for compassionate conservation (pp. 379-388). Chicago, IL: University of Chicago Press.

Bekoff, M. (2014, December 18). On killing wolves and other animals: Should only trained ethicists weigh in? Huffington Post. Retrieved from http://www.huffingtonpost.com/marc-bekoff/on-killing-wolves-andoth_b_6340296.html

Bekoff, M., \& Pierce, J. (2009). Wild justice: The moral lives of animals. Chicago, Il: University of Chicago Press.

Bell, A. C., \& Russell, C. L. (1999). Life ties: Disrupting anthropocentrism in language arts education. In J. P. Robertson (Ed.), Teaching for a tolerant world, grades $K$ 6: Essays and resources (pp. 68-89). Urbana, IL: National Council of Teachers of English.

Bell, A. C., \& Russell, C. L. (2000). Beyond human, beyond words: Anthropocentrism, critical pedagogy, and the poststructuralist turn. Canadian Journal of Education, 25(3), 188-203.

Bell, A. C., Russell, C. L., \& Plotkin, R. (1998). Environmental learning and the study of extinction. Journal of Environmental Education, 29(2), 4-10.

Berg, B. L. (2007). Qualitative research methods for the social sciences (6 ${ }^{\text {th }}$ ed.). Boston, MA: Pearson.

Bisi, J., Kurki, S., Svensberg, M., \& Liukkonen, T. (2007). Human dimensions of wolf (Canis lupus) conflicts in Finland. European Journal of Wildlife Research, 53(4), 304-314.

Bizerril, M. X. A., Soares, C. C., \& Santos, J. P. (2011). Linking community communication to conservation of the maned wolf in central Brazil. Environmental Education Research, 17(6), 815-827.

Black, P., \& Rutberg, A. (2007). Effectiveness of gray wolf (Canis Lupus) education programs in the midwest: A case study of Wolf Park. Endangered Species Update, 24(1), 3. 
Bogdan, R. C., \& Biklen, S. K. (1998). Qualitative research for education: An introduction to theory and methods $\left(3^{\text {rd }}\right.$ ed.). Needham Heights, MA: Allyn \& Bacon.

Bogner, F. X. (1998). The influence of short-term outdoor ecology education on longterm variables of environmental perspective. Journal of Environmental Education, 29(4), 17-29.

Boitani, L. (1995). Ecological and cultural diversities in the evolution of wolf-human relationships. In L. N. Carbyn, S. H. Fritts, \& D. R. Seip (Eds.), Ecology and conservation of wolves in a changing world (pp. 3-12). Edmonton, AB: Canadian Circumpolar Institute.

Boitani, L. (2003). Wolf conservation and recovery. In L. D. Mech \& L. Boitani (Eds.), Wolves: Behavior, ecology, and conservation (pp. 317-340). Chicago, IL: University of Chicago Press.

Breunig, M. (2005). Turning experiential education and critical pedagogy theory into practice. Journal of Experiential Education, 28(2), 106-122.

Bruskotter, J. T., Schmidt, R. H., \& Teel, T. L. (2007). Are attitudes towards wolves changing? A case study in Utah. Biological Conservation, 139(1/2), 211-218.

Bruskotter, J. T., Toman, E., Enzler, S. A., \& Schmidt, R. H. (2010). Gray wolves not out of the woods yet. Science, 327(5961), 30-31.

Bruskotter, J. T., Vucetich, J. A., Enzler, S., Treves, A., \& Nelson, M. P. (2014). Removing protections for wolves and the future of the U.S. endangered species act (1973). Conservation Letters, 7(4), 401-407.

Butler, L., Dale, B., Beckmen K., \& Farley, S. (2011). Findings related to the March 2010 fatal wolf attack near Chignik Lake, Alaska (Wildlife Special Publication, ADF\&G/DWC/WSP-2011-2). Palmer, Alaska: Department of Fish and Game.

Canadian Institutes of Health Research, Natural Sciences and Engineering Research Council of Canada, and Social Sciences and Humanities Research Council of Canada. (2010). Tri-Council Policy Statement: Ethical Conduct for Research Involving Humans. Ottawa: Authors.

Capra, F. (2007). Sustainable living, ecological literacy, and the breath of life. Canadian Journal of Environmental Education, 12, 9-18.

Caro, T., Mulder, M. B., \& Moore, M. (2003). Effects of conservation education on reasons to conserve biological diversity. Biological Conservation, 114(1), 143152. 
Case, L. (2008). Perspectives on domestication: The history of our relationship with man's best friend. Journal of Animal Science, 86(11), 3245-3251.

Castellano, M., Quirino de Luca, A., \& Sorrentino, M. (2011). The interface of environmental and humane education as an emerging and relevant dialogue: A point of view from Brazil. Canadian Journal of Environmental Education, 16, 93-105.

Chadwick, D. H. (2010, March). Wolf wars. National Geographic, 34-55.

Chapron, G., Kaczensky, P., Linnell, J. D. C., Arx, M., Huber, D., Andrén, H., ...Boitani, L. (2014). Recovery of large carnivores in Europe's modern human-dominated landscapers. Science, 346(6216), 1517-1519.

Claxton, A. M. (2011). The potential of the human-animal relationship as an environmental enrichment for the welfare of zoo-housed animals. Applied Animal Behaviour Science, 133(1), 1-10.

Colorado Wolf and Wildlife Center. (n. d.). Our mission statement. Retrieved from http://www.wolfeducation.org/\#!our-mission/cfll

Corman, L. (2011). Impossible subjects: The figure of the animal in Paulo Freire's Pedagogy of the Oppressed. Canadian Journal of Environmental Education, 16, 29-45.

Creel, S., \& Rotella, J. J. (2010). Meta-analysis of relationships between human offtake, total mortality and population dynamics of gray wolves (Canis lupus). PLoS ONE, 5(9), e129118. Retrieved from http://www.plosone.org/article/fetchObject.action?uri=info:doi/10.1371/journal.p one.0012918\&representation $=\mathrm{PDF}$

Creswell, J. W. (2012). Educational research: Planning, conducting, and evaluating quantitative and qualitative research ( $4^{\text {th }}$ ed.). Upper Saddle River, NJ: Pearson.

Darder, A., Baltodano, M. P., \& Torres, R. D. (2009). Critical pedagogy: An introduction. In A. Darder, M. P. Baltodano, \& R. D. Torres (Eds.), The critical pedagogy reader ( $2^{\text {nd }}$ ed., pp. 522-540). New York, NY: Routledge.

de White, T. G., \& Jacobson, S. K. (1994). Evaluating conservation education programs at a South American zoo. Journal of Environmental Education, 25(4), 18-22.

Dettmann-Easler, D., \& Pease, J. (1999). Evaluating the effectiveness of residential environmental education programs in fostering positive attitudes toward wildlife. Journal of Environmental Education, 31(1), 33-39. 
Dierking, L. D., Falk, J. H., \& Storksdieck, M. (2013). Learning from neighboring fields: Conceptualizing outcomes of environmental education within the framework of free-choice learning experiences. In R. B. Stevenson, M. Brody, J. Dillon, \& A. E. J. Wals (Eds.), International handbook of research on environmental education (pp. 359-366). New York, NY: Routledge.

Dillon, J., Rickinson, M., Tearney, K., Morris, M., Choi, M. Y., Sanders, D., \& Benefield, P. (2006). The value of outdoor learning: Evidence from research in the UK and elsewhere. School Science Review, 87(320), 107-111.

Edmonton AM CBC News (2015, October 19). Wolf-culling policies need updating, Alberta conservationist says. CBC News. Retrieved from http://www.cbc.ca/news/canada/edmonton/wolf-culling-policies-need-updatingalberta-conservationist-says- 1.3278440

Eisenberg, C. (2010). The wolf's tooth: Keystone predators, trophic cascades, and biodiversity. Washington, DC: Island Press.

Eisenberg, C. (2014). The carnivore way: Coexisting with and conserving North American's predators. Washington, D.C.: Island Press.

Eisner, E. (1997). The promise and the perils of alternative forms of data representation. Educational Researcher, 26(6), 4-10.

Ellison, G. (2015, September 3). Import wolves to Michigan's Isle Royale 'as soon as possible,' says biologist. Mlive. Retrieved from http://www.mlive.com/news/index.ssf/2015/09/isle_royale_wolves_moose_plan.h tml

Emel, J. (1995). Are you man enough, big and bad enough? Ecofeminism and wolf eradication in the USA. Environment and Planning D: Society and Space, 13(6), 707-734.

Emel, J., Wilbert, C., \& Wolch, J. (2002). Animal geographies. Society \& Animals, 10(4), 407-412.

Enck, J. W., \& Brown, T. L. (2002). New Yorkers' attitudes toward restoring wolves to the Adirondack Park. Wildlife Society Bulletin, 30(1), 16-28.

Ericsson, G., \& Heberlein, T. A. (2003). Attitudes of hunters, locals, and the general public in Sweden now that the wolves are back. Biological Conservation, 111(2), 149-159.

Evans, E., Ching, C. C., \& Ballard, H. L. (2012). Volunteer guides in nature reserves: exploring environmental educators' perceptions of teaching, learning, place and self. Environmental Education Research, 18(3), 391-402. 
Evernden, N. (1985). The natural alien: Humankind and environment (2nd ed.). Toronto, Ontario: University of Toronto Press.

Falk, J. H., Reinhard, E. M., Vernon, C. L., Bronnenkant, K., Heimlich, J. E., \& Deans, N. L. (2007). Why zoos and aquariums matter: Assessing the impact of a visit to a zoo or aquarium. Silver Spring, MD: Association of Zoos and Aquariums.

Fawcett, L. (1989). Anthropomorphism: In the web of culture. Undercurrents, 1, 14-20.

Fawcett, L. (2000). Ethical imagining: Ecofeminist possibilities and environmental learning. Canadian Journal of Environmental Education, 5, 134-149.

Fawcett, L. (2002). Children's wild animal stories: Questioning inter-species bonds. Canadian Journal of Environmental Education, 7(2), 125-139.

Fawcett, L. (2013). Three degrees of separation: Accounting for naturecultures in environmental education research. In R. B. Stevenson, M. Brody, J. Dillon, \& A. E. J. Wals (Eds.), International handbook of research on environmental education (pp. 409-417). New York, NY: Routledge.

Fidler, M., Light, P., \& Costall, A. (1996). Describing dog behavior psychologically: Pet owners versus non-owners. Anthrozoös, 9(4), 196-200.

Friends of Algonquin Park (2014). Wolf howls. Retrieved from http://www.algonquinpark.on.ca/visit/programs/wolf-howls.php

Fritts, S. H., Stephenson, R. O., Hayes, R. D., \& Boitani, L. (2003). Wolves and humans. In L. D. Mech \& L. Boitani (Eds.), Wolves: Behavior, ecology, and conservation (pp. 289-316). Chicago, Il: University of Chicago Press.

Fox, C. H., \& Bekoff, M. (2009). Ethical reflections on wolf recovery and conservation: A practical approach for making room for wolves. In M. Musiani, L. Boitani, \& P. C. Paquet (Eds.), A new era for wolves and people: Wolf recovery, human attitudes, and policy (pp. 117-139). Calgary, Alberta: University of Calgary Press.

Gerbasi, K. C., Anderson, D. C., Gerbasi, A. M., \& Coultis, D. (2002). Doctoral dissertations in human-animal studies: News and views. Society \& Animals, 10(4), 339-346.

Giroux, H. A., \& Giroux, S. S. (2006). Challenging neoliberalism's new world order: The promise of critical pedagogy. Cultural Studies $\leftrightarrow$ Critical Methodologies, $6(1), 21-32$. 
Glick, M. P., \& Samarapungavan, A. (2008). Wolves are beautiful and proud: Science learning from a school field trip. Journal of Museum Education, 33(2), 199-208.

Gough, A. (2013). The emergence of environmental education research: A 'history' of the field. In R. B. Stevenson, M. Brody, J. Dillon, \& A. E. J. Wals (Eds.), International handbook of research on environmental education (pp. 13-22). New York, NY: Routledge.

Greenwood, D. A. (2010). Nature, empire, and paradox in environmental education. Canadian Journal of Environmental Education, 15, 9-24.

Groenewald, T. (2004). A phenomenological research design illustrated. International Journal of Qualitative Methods, 3(1), 1-25.

Gruen, L. (1993). Dismantling oppression: An analysis of the connection between women and animals. In G. Gaard (Ed.), Ecofeminism: Women, animals, nature (pp. 60-90). Philadelphia, PA: Temple University Press.

Gruenwald, D. A. (2008). The best of both worlds: A critical pedagogy of place. Environmental Education Research, 14(3), 308-324.

Haluza-DeLay, R. (2013). Educating for environmental justice. In R. B. Stevenson, M. Brody, J. Dillon, \& A. E. J. Wals (Eds.), International handbook of research on environmental education (pp. 394-403). New York, NY: Routledge.

Harper, D. (2002). Talking about pictures: A case for photo-elicitation. Visual Studies, 17(1), 13-26.

Hart, P., \& Nolan, K. (1999). A critical analysis of research in environmental education. Studies in Science Education, 34(1), 1-69.

Hodson, D. (2010). Science education as a call to action. Canadian Journal of Science, Mathematics and Technology Education, 10(3), 197-206.

Hope, J. (1994). Wolves and wolf hybrids as pets are big business - but a bad idea. Smithsonian, 25(3), 34.

Houston, M. J., Bruskotter, J. T., \& Fan, D. (2010). Attitudes toward wolves in the United States and Canada: A content analysis of the print news media, 1999-2008. Human Dimensions of Wildlife, 15(5), 389-403.

Humes, B. (2008). Moving toward a liberatory pedagogy for all species: Mapping the need for dialogue between humane and anti-oppressive education. Green Theory \& Praxis: The Journal of Ecopedagogy, 4(1), 65-85. 
Irvine, L. (2004). If you tame me: Understanding our connections with animals. Philadelphia, PA: Temple University Press.

Jickling, B. (2005a). "The wolf must not be made a fool of": Reflections on education, ethics and epistemology. In P. Tripp \& L. Muzzin (Eds.), Teaching as activism: Equity meets environmentalism (pp. 35-45). Montreal, PQ: McGill-Queens's University Press.

Jickling, B. (2005b). Education and advocacy: A troubling relationship. In E. A. Johnson \& M. Mappin (Eds.), Environmental education and advocacy: Changing perspectives of ecology and education (pp. 91-113). Cambridge, U.K.: Cambridge University Press.

Jickling, B., \& Paquet, P. C. (2005). Wolf stories: Reflections on science, ethics, and epistemology. Environmental Ethics, 27(2), p. 115-134.

Jobin, B. (2007, November 2). Wolves killed student: Jury. Prince Albert Daily Herald. Retrieved from http://www.paherald.sk.ca/Education/2007-11-02/article178031/Wolves-killed-student-jury/1

Johnson, J. M., \& Rowlands, T. (2012). The interpersonal dynamics of in-depth interviewing. In J. F. Gubrium, J. A. Holstein, A. B. Marvasti, \& K. D. McKinny (Eds.), The Sage handbook of interview research: The complexity of the Craft $\left(2^{\text {nd }}\right.$ ed., pp. 99-113). Los Angeles, CA: Sage.

Kahn, R. (2009). Towards ecopedagogy: Weaving a broad-based pedagogy of liberation for animals, nature, and the oppressed people of the earth. In A. Darder, M. P. Baltodano, \& R. D. Torres (Eds.), The critical pedagogy reader ( ${ }^{\text {nd }}$ ed., pp. 522540). New York, NY: Routledge.

Kahn, R., \& Humes, B. (2009). Marching out from Ultima Thule: Critical counterstories of emancipatory educators working at the intersection of human rights, animal rights, and planetary sustainability. Canadian Journal of Environmental Education, 14, 179-195.

Karlsson, J., \& Sjöström, M. (2007). Human attitudes towards wolves: A matter of distance. Biological Conservation, 137(4), 610-616.

Kellert, S. R., Black, M., Rush, C. R., \& Bath, A. J. (1996). Human culture and large carnivore conservation in North America. Conservation Biology, 10(4), 977-990.

Kelsey, E., \& Armstrong, C. (2012). Finding hope in a world of environmental catastrophe. In: A. E. J. Wals \& P. B. Corcoran (Eds.), Learning for sustainability in times of accelerating change (pp. 187-200). Wageningen, The Netherlands: Wageningen Academic Publishers. 
Kirova, A., \& Emme, M. (2006). Using photography as a means of phenomenological seeing:" Doing phenomenology" with immigrant children. Indo-Pacific Journal of Phenomenology: Methodology: Special Edition, 6, 1-12.

Kollmuss, A., \& Agyeman, J. (2002). Mind the gap: Why do people act environmentally and what are the barriers to pro-environmental behavior? Environmental Education Research, 8(3), 239-260.

Kuhl, G. J., (2008). Human-sled dog relations: What can we learn from the stories and experiences of mushers? (Master's thesis). Retrieved from ProQuest Dissertations and Theses Database. (UMI No. MR43424)

Kuhl, G. (2011a). Human-sled dog relations: What can we learn from the stories and experiences of mushers? Society \& Animals, 19(1), 22-37.

Kuhl, G. J. (2011b). Representing animal-others in educational research. Canadian Journal of Environmental Education, 16, 106-122.

Leopold, A. (1949). A Sand County almanac, and sketches here and there. New York, NY: Oxford University Press.

Lichtman, M. (2010). Qualitative research in education: A user's guide (2 ${ }^{\text {nd }}$ ed.). Los Angeles, CA: Sage.

Lichtman, M. (2013). Qualitative research in education: A user's guide (3 ${ }^{\text {rd }}$ ed.). Los Angeles, CA: Sage.

Liddicoat, K., \& Krasny, M. E. (2013). Research on the long-term impacts of environmental education. In R. B. Stevenson, M. Brody, J. Dillon, \& A. E. J. Wals (Eds.), International handbook of research on environmental education (pp. 289-297). New York, NY: Routledge.

Livingston, J. A. (1981). The fallacy of wildlife conservation. Toronto, ON: McClelland $\&$ Stewart.

Lloro-Bidart, T. \& Russell, C. (2016, in press). Learning science in aquariums and on whalewatching boats: The hidden curriculum of the deployment of other animals. In M. Mueller, D. J. Tippins, \& A. J. Stewart (Eds.), Animals and science education (pp. tba). New York: Springer.

Lodico, M. G., Spaulding, D. T., \& Voegtle, K. H. (2006). Methods in educational research: From theory to practice. San Francisco, CA: John Wiley \& Sons.

Lohr, C., Ballard, W. B., \& Bath, A. (1996). Attitudes toward gray wolf reintroduction to New Brunswick. Wildlife Society Bulletin, 24(3), 414-420. 
Lopez, B. H. (1978). Of wolves and men. New York, NY: Charles Scribner's Sons.

Lynn, W. S. (2002). Canis Lupus cosmopolis: Wolves in a cosmopolitan worldview. Worldviews: Global Religions, Culture, and Ecology, 6(3), 300-327.

Lynn, W. S. (2007). Ethics and animal protection: Wolf recovery. In M. Bekoff (Ed.), Encyclopedia of human-animal relationships: A global exploration of our connections with animals (Vol. 3, pp. 812-819). Westport, CT: Greenwood Press.

Lynn, W. S. (2010). Discourse and wolves: Science, society, and ethics. Society \& Animals, 18(1), 75-92.

MacPherson, S. (2011). What Tashi taught me: "Petagogy" and the education of emotions. Canadian Journal of Environmental Education, 16, 76-92.

Magrini, J. (2012). Phenomenology for educators: Max van Manen and "human science" research. Philosophy Scholarship. Paper 32. Retrieved from http://dc.cod.edu/philosophypub/32

Marino, L., Lilienfeld, S. O., Malamud, R., Nobis, N., \& Broglio, R. (2010). Do zoos and aquariums promote attitude change in visitors? A critical evaluation of the American zoo and aquarium study. Society \& Animals, 18(2), 126-138.

Martin, P. (2007). Caring for the environment: Challenges from notions of caring. Australian Journal of Environmental Education, 23, 57-63.

Mason, M. (2010). Sample size and saturation in $\mathrm{PhD}$ studies using qualitative interviews. Forum Qualitative Sozialforschung/Forum: Qualitative Social Research, 11(3), Art. 8. Retrieved from http://nbn-resolving.de/urn:nbn:de:0114fqs 100387.

McNay, M. E. (2002). Wolf-human interactions in Alaska and Canada: A review of the case history. Wildlife Society Bulletin, 30(3), 831-843.

Mead, G. H. (1962). Mind, self, and society. Chicago, IL: University of Chicago.

Midgley, M. (2001). The problem of living with wildness. In V. A. Sharpe, B. G. Norton, \& S. Donnelley (Eds.), Wolves and human communities (pp. 179-190). Washington, DC: Island Press.

Mony, P. R. S., \& Heimlich, J. E. (2008). Talking to visitors about conservation: Exploring message communication through docent-visitor interactions at zoos. Visitor Studies, 11(2), 151-162.

Morell, V. (2008). Wolves at the door of a more dangerous world. Science, 319(5865), 890-892. 
Mortari, L. (2004). Educating to care. Canadian Journal of Environmental Education, 9(1), 109-122.

Murray, D. L., Smith, D. W., Bangs, E. E., Mack, C., Oakleaf, J. K, Fontaine, J., ...Asher, V. J. (2010). Death from anthropogenic causes is partially compensatory in recovering wolf populations. Biological Conservation, 143(11), 2514-2524.

Musiani, M., Boitani L., \& Paquet, P. C. (2009). Introduction: Newly recovering wolf populations produce new trends in human attitudes and policy. In M. Musiani, L. Boitani, \& P. C. Paquet (Eds.), A new era for wolves and people: Wolf recovery, human attitudes, and policy (pp. 1-12). Calgary, AB: University of Calgary Press.

Musiani, M., \& Paquet, P. C. (2004). The practices of wolf persecution, protection, and restoration in Canada and the United States. BioScience, 54(1), 50-60.

Naughton-Treves, L., Grossberg, R., \& Treves, A. (2003). Paying for tolerance: Rural citizens'attitudes toward wolf depredation and compensation. Conservation Biology, 17(6), 1500-1511.

Nespor, J. (2000). Anonymity and place in qualitative inquiry. Qualitative Inquiry, 6(4), 546-569.

Nie, M. A. (2002). Wolf recovery and management as value-based political conflict. Ethics, Place and Environment, 5(1), 65-71.

Noske, B. (1997). Beyond boundaries: Humans and animals. Montreal, PQ: Black Rose Books.

Oakley, J., Watson, G. P. L., Russell, C. L., Cutter-Mackenzie, A. Fawcett, L., Kuhl, G., Russell, J., van der Waal, M., \& Warkentin, T. (2010). Animal encounters in environmental education research: Responding to the "question of the animal." Canadian Journal of Environmental Education, 15, 102-118.

Ohlson, D., Cushing, K., Trulio, L., \& Leventhal, A. (2008). Advancing Indigenous selfdetermination through endangered species protection: Idaho Gray wolf recovery. Environmental Science and Policy, 11(5), 430-440.

Ontario Ministry of Natural Resources (2005). Backgrounder on wolf conservation in Ontario. Toronto, ON: Ontario Ministry of Natural Resources.

Orams, M. B. (1997). The effectiveness of environmental education: Can we turn tourists into 'greenies'? Progress in Tourism and Hospitality Research, 3(4), 295-306. 
Orr, D. W. (1994). Earth in mind: On education, environment, and the human prospect. Washington, DC: Island Press.

Oulton, C., Dillon, J., \& Grace, M. M. (2004). Reconceptualizing the teaching of controversial issues. International Journal of Science Education, 26(4), 411-423.

Palmer, J. A. (1998). Environmental education in the $21^{\text {st }}$ century: Theory, practice, progress and promise. London, UK: RoutledgeFalmer.

Pedersen, H. (2010). Animals in schools: Processes and strategies in human-animal education. West Lafayette, IN: Purdue University Press.

Pedersen, H. (2011). Counting affects: Mo(ve)ments of intensity in critical avian education. Canadian Journal of Environmental Education, 16, 14-28.

Pedretti, E., \& Nazir, J. (2011). Currents in STSE education: Mapping a complex field, 40 years on. Science Education, 95(4), 601-626.

Pets by the Numbers. (2015). Retrieved from

http://www.humanesociety.org/issues/pet_overpopulation/facts/pet_ownership_sta tistics.html

Plunkett, R., Leipert, B. D., \& Ray, S. L. (2013). Unspoken phenomena: using the photovoice method to enrich phenomenological inquiry. Nursing Inquiry, 20(2), 156-164

Prokop, P., \& Tunnicliffe, S. D. (2010). Effects of having pets at home on children's attitudes toward popular and unpopular animals. Anthrozoös, 23(1), 21-35.

Prokop, P., Usak, M., \& Erdogan, M. (2011). Good predators in bad stories: Crosscultural comparison of children's attitudes towards wolves. Journal of Baltic Science Education, 10(4), 229-242.

Ripple, W. J., \& Beschta, R. L. (2004). Wolves and the ecology of fear: Can predation risk structure ecosystems? Bioscience, 54(8), 755-766.

Ripple, W. J., \& Beschta, R. L. (2005). Linking wolves and plants: Aldo Leopold on trophic cascades. BioScience, 55(7), 613-621.

Ripple, W. J., Estes, J. A., Beschta, R. L., Wilmers, C. C., Ritchie, E. G., Hebblewhite, M.,...Wirsing, A. J. (2014). Status and ecological effects of the world's largest carnivores. Science, 343(6167), 1241484.

Røskaft, E. Bjerke, T., Kaltenborn, B., Linnell, J. D. C., \& Andersen, R. (2003). Patterns of self-reported fear towards large carnivores among the Norwegian public. Evolution and Human Behavior, 24(3), 184-198. 
Ross, N., Medin, D., Coley, J. D., \& Atran, S. (2003). Cultural and experiential differences in the development of folkbiological induction. Cognitive Development, 18(1), 25-47.

Russell, C. L. (1997). Approaches to environmental education: Toward a transformative perspective. Holistic Education Review, 10(1), 34-40.

Russell, C. L. (1999). Problematizing nature experience in environmental education: The interrelationship of experience and story. Journal of Experiential Education, 22(3), 123-128, 137.

Russell, C. L. (2001a). Why study whalewatching? Environmental education, nature experience, and the social construction of nature. In D. Hodson (Ed.), OISE papers in STSE education (Vol. 2, pp. 49-74). Toronto, ON: University of Toronto Press.

Russell, C. L. (2001b). Tales of whales: Whalewatching as environmental education? (Unpublished doctoral dissertation). University of Toronto, Toronto.

Russell, C. L. (2003). Minding the gap between methodological desires and practice. In D. Hodson (Ed.), OISE papers in STSE education, Volume 4 (pp. 125-134). Toronto, University of Toronto Press.

Russell, C. L. (2005). 'Whoever does not write is written': The role of 'nature' in postpost approaches to environmental education research. Environmental Education Research, 11(4), 433-443.

Russell, C. L., \& Bell, A. C. (1996). A politicized ethic of care: Environmental education from an ecofeminist perspective. In K. Warren (Ed.), Women's voices in experiential education (pp. 172-181). Dubuque, IA: Kendall/Hunt.

Russell, C. L., \& Fawcett, L. (2013). Moving margins in environmental education research. In R. B. Stevenson, M. Brody, J. Dillon, \& A. E. J. Wals (Eds.), International handbook of research on environmental education (pp. 369-374). New York, NY: Routledge.

Russell, C. L., \& Hodson, D. (2002). Whalewatching as critical science education? Canadian Journal of Science, Mathematics and Technology Education, 2(4), 485504.

Samuels, W. E. (2007). Education: Nurturing empathy in children. In M. Bekoff (Ed.), Encyclopedia of human-animal relationships: A global exploration of our connections with animals (Vol. 2, pp. 680-684). Westport, CT: Greenwood Press. 
Samuelson, M. M. (2012). The effects of visiting a Montana wildlife conservation center on attitudes towards grey wolves (canis lupus) (Unpublished master's thesis). University of Idaho, Moscow, Idaho.

Sanders, C. R. (1999). Understanding dogs: Living and working with canine companions. Philadelphia, PA: Temple University Press.

Sauvé, L. (2005). Currents in environmental education: Mapping a complex and evolving pedagogical field. Canadian Journal of Environmental Education, 10, 11-37.

Scarce, R. (1998). What do wolves mean? Conflicting social constructions of Canis lupus in "Bordertown". Human Dimensions of Wildlife, 3(3), 26-45.

Schlickeisen, R. (2001). Overcoming cultural barriers to wolf reintroduction. In V. A. Sharpe, B. G. Norton, \& S. Donnelley (Eds.), Wolves and human communities (pp. 61-74). Washington, DC: Island Press.

Seidman, I. (2013). Interviewing as qualitative research: A guide for researchers in education and the social sciences $\left(4^{\text {th }}\right.$ ed.). New York, NY: Teachers College Press.

Selby, D. (1995). Earthkind: A teachers' handbook on humane education. Staffordshire, England: Trentham Books.

Selby, D. (2000). A darker shade of green: The importance of ecological thinking in global education and school reform. Theory into Practice, 39(2), 88-96.

Shapiro, K. (1997). A phenomenological approach to the study of nonhuman animals. In R. W. Mitchell, R. S. Thompson, \& H. L. Miles (Eds.), Anthropomorphism, anecdotes, and animals (pp. 277-295). Albany, NY: State University of New York Press.

Shapiro, K. (2002). Editor's introduction: The state of human-animal studies: Solid, at the margin! Society \& Animals, 10(4), 331-337.

Shelley, V. Treves, A., \& Naughton, L. (2014). Attitudes to wolves and wolf policy among Ojibwe tribal members and non tribal residents of Wisconsin's wolf range. Human Dimensions of Wildlife, 16(6), 397-314.

Skogen, K. (2001). Who's afraid of the big bad wolf? Young people's responses to the conflict over large carnivores in Eastern Norway. Rural Sociology, 66(2), 203226.

Skogen, K., Mauz, I., \& Krange, O. (2008). Cry wolf!: Narratives of wolf recovery in France and Norway. Rural Sociology, 73(1), 105-133. 
Skogen, K., \& Thrane, C. (2008). Wolves in context: Using survey data to situate attitudes within a wider cultural framework. Society and Natural Resources, 21(1), 17-33.

Smith, D. W. (2007). Wolf and human conflicts: A long, bad history. In M. Bekoff (Ed.), Encyclopedia of human-animal relationships: A global exploration of our connections with animals (Vol. 2, pp. 402-409). Westport, CT: Greenwood Press.

Smith, J. (2006). Wild wolves? Understanding human-wolf interactions in a coastal Canadian national park reserve. Unpublished master's thesis, Lakehead University, Thunder Bay, Ontario, Canada.

Sponarski, C. C., Semeniuk, C., Glikman, J. A., Bath, A. J., \& Musiani, M. (2013). Heterogeneity among rural resident attitudes toward wolves. Human Dimensions of Wildlife, 18(4), 239-248.

Stevenson, R. B., Dillon, J., Wals, A. E. J., \& Brody, M. (2013). The evolving characteristics of environmental education research. In R. B. Stevenson, M. Brody, J. Dillon, \& A. E. J. Wals (Eds.), International handbook of research on environmental education (pp. 512-517). New York, NY: Routledge.

Stevenson, R. B., Wals, A. E. J., Dillon, J., \& Brody, M. (2013). Introduction: An orientation to environmental education and the handbook. In R. B. Stevenson, M. Brody, J. Dillon, \& A. E. J. Wals (Eds.), International handbook of research on environmental education (pp. 1-12). New York, NY: Routledge.

Switalski, T. A., Simmons, T., Duncan, S. L., Chavez, A. S., \& Schmidt, R. H. (2002). Education and public involvement for wolves in Utah. Natural Resources and Environmental Issues, 10, 36-40.

Talmazan, Y. (2015, September 24). National Geographic puts spotlight on B.C.'s enigmatic sea wolves. Global News. Retrieved from http://globalnews.ca/news/2239088/national-geographic-puts-spotlight-on-b-c-senigmatic-sea-wolves/

Taylor, D. (2009). Education as a conservation strategy: Exploring perspective transformation. In M. Musiani, L. Boitani, \& P. C. Paquet (Eds.), A new era for wolves and people: Wolf recovery, human attitudes, and policy (pp. 161-171). Calgary, AB: University of Calgary Press.

Taylor, E. W., \& Caldarelli, M. (2004). Teaching beliefs of non-formal environmental educators: A perspective from state and local parks in the United States. Environmental Education Research, 10(4), 451-469. 
Thalmann, O., Shapiro, B., Cui, P., Schuenemann, V. J., Sawyer, S. K., Greenfield, D. L.,...Wayne, R. K. (2013). Complete mitochondrial genomes of ancient canids suggest a European origin of domestic dogs. Science, 342(6160), 871-874.

Theberge, J. B., \& Theberge, M. T. (2004). The wolves of Algonquin Park: A 12 year ecological study. Waterloo, ON: Department of Geography University of Waterloo.

Thomas, E. (2001). The two-leggeds and the four-leggeds. In V. A. Sharpe, B. G. Norton, \& S. Donnelley (Eds.), Wolves and human communities (pp. 47-49). Washington, DC: Island Press.

Thomas, D. R. (2006). A general inductive approach for analyzing qualitative evaluation data. American Journal of Evaluation, 27(2), 237-246.

Todd, S. (2002). Building consensus on diverse issues: A case study of the Yukon wolf management team. Environment Impact Assessment Review, 22(6), 655-684.

Treves, A., \& Bruskotter, J. T. (2011). Gray wolf conservation at a crossroads. BioScience, 61(8), 584-585.

Troxell, P.S., Berg, K.A., Jaycox, H., Strauss, A. L. Struhsacker, P., \& Callahan, P. (2009). Education and outreach efforts in support of wolf conservation in the Great Lakes region. In A. P. Wydeven, T.R. Deelen, \& E. Heske (Eds.), Recovery of Gray wolves in the Great Lakes region of the United States (pp. 297-309). New York, NY: Springer.

Vagle, M. E. (2014). Crafting phenomenological research. Walnut Creek, CA: Left Coast Press.

Van Manen, M. (2002). Writing in the dark: Phenomenological studies in interpretive inquiry. London, ON: Althouse Press.

Van Manen, M. (2014). Phenomenology of practice: Meaning-giving methods in phenomenological research and writing. Walnut Creek, CA: Left Coast Press.

Visscher, N. C., Snider, R., \& Stoep, G. V. (2009). Comparative analysis of knowledge gain between interpretive and fact-only presentations at an animal training session: An exploratory study. Zoo Biology, 28(5), 488-495.

Wals, A. E., Brody, M., Dillon, J., \& Stevenson, R. B. (2014). Convergence between science and environmental education. Science, 344(6184), 583-584.

Warkentin, T. (2007). Captive imaginations: Affordances for ethics, agency and knowledge-making in whale-human encounters. (Doctoral Dissertation). Retrieved from ProQuest Dissertations and Theses Database. (UMI No. NR32073) 
Warkentin, T. (2011). Interspecies etiquette in place: Ethical affordances in swim-withdolphins programs. Ethics and The Environment, 16(1), 99-122.

Watson, G. P. L. (2006). Wild becomings: How the everyday experience of common wild animals at summer camp acts as an entrance to the more-than-human world. Canadian Journal of Environmental Education, 11, 127-142.

Weil, Z. (2004). The promise and power of humane education. Gabriola Island, BC: New Society Publishers.

White, A. B. (2014). Captive wolf facilities in North America. Retrieved from http://www.graywolfconservation.com/Captivity/seeing_captive_wolves.ht

Wild Spirit Wolf Sanctuary, (n. d.). Our mission. Retrieved from http://www.wildspiritwolfsanctuary.org/mission.php

Willard, A. L. (2008). Presenting wolves as wolves: Educational outreach in the debate about wolf management in the west. (Master's thesis, Washington State University), Retrieved from http://www.dissertations.wsu.edu/Thesis/Fall2008/a_willard_111408.pdf

Williams, C. K., Ericsson, G., \& Heberlein, T. A. (2002). A quantitative summary of attitudes toward wolves and their reintroduction (1972-2000). Wildlife Society Bulletin, 30(2), 575-584.

Wolf Conservation Center. (2015). About us. Retrieved from http://nywolf.org/about

Wolf Haven International (2014). Conservation. Retrieved from http://www.wolfhaven.org/conservation/

Wolf Park. (2015). Mission. Retrieved from http://wolfpark.org/about/mission/ 


\section{Appendix A: Possible Interview Questions for Wolf Educators}

- How/why did you become a wolf educator?

- Did you have any experiences with being an educator prior to your present work?

- What were your beliefs about wolves prior to becoming a wolf educator?

- Did you grow up in a region where wolves reside?

- Had you had any experiences/encounters with wolves before working here?

- What types of tasks does your job entail? Describe some of them.

- What is/are your favourite part/s of working for this program?

- What have been the biggest challenges for you as a wolf educator?

- Is wolf survival a contentious issue in the area/region where you work?

- What types of visitors/participants come to your programs/centre?

- What do you find most interesting about educating people about wolves?

- How are wolves similar/different from people?

- What is your favourite part of working with wolves?

- Have you learned anything specifically through interacting with wolves?

- Have the wolves themselves taught you anything?

- Do you have any interesting stories about your time spent with wolves?

- Have there been any surprises for you either as an educator or someone who interacts with wolves?

- Do you believe wolf survival is important? Why/why not?

- Do you think wolves will survive in the region where you work? Why? Why not?

- Do you think education can make a difference when it comes to wolf survival?

- What do you believe is the place for wolves in our world? 
- What do you believe are the biggest challenges for wolf survival on the planet?

- What have been your biggest personal learnings about wolves? About wolf education?

- What have you learned about wolves you would like the general public to understand? 


\section{Appendix B: Cover Letter}

Dear Potential Participant:

My name is Gail Kuhl and I am conducting a study in partial fulfillment of a $\mathrm{PhD}$ in Education at Lakehead University in Thunder Bay, Ontario and funded though a fellowship from the Social Sciences and Humanities Research Council of Canada (SSHRC). The title of my study is "Living Well in a World with Wolves: Educators' Perspectives". Through interviewing educators, I hope to learn, "What are the experiences of educators who work at programs that feature live wolves; in particular, what do they learn through both working with and teaching about wolves?"

While there are no direct benefits to you if you choose to participate, your knowledge and experience may offer insight into what happens at programs dedicated to educating the public about wolves. I also believe we need to better understand the relations between species; therefore, I would be interested to hear about your experiences and learnings both teaching about, and working with, wolves.

Your participation in my study would mean volunteering your time (in person or by telephone or Skype) for an interview of approximately an hour. I would ask you a number of questions about your experiences working as a wolf educator (there are no wrong answers). You can choose to either answer, or not answer, any of the questions I ask during the interview at your discretion. The discussion during the interview would be fairly unstructured; you would be free to offer any insights about the topic that you feel are relevant. With your consent, I will record the interview so I can transcribe and analyse it afterwards. You can request a copy of the transcript and change or adjust anything you wish.

I am also interested in observing several educators at work. If you would be willing to have me observe you for a few hours once or twice while you are teaching about and interacting with the wolves at your program, please let me know.

Unless you state otherwise, the information you share with me will remain anonymous and I will use a pseudonym (fake name) of your choice in both my dissertation and any other material I publish from this study. However, be aware that if people are familiar with specific details of the program where you work, it is possible that those who read my research in the future may be able to identify you.

If you have any photos of yourself interacting with wolves, and are willing, we could use them as discussion points during the interview. Similarly, if I am invited to observe you working at your program, and you agree, I could take some photos for us to use as discussion points during the interview. There is a consent form for photos (attached) where you can indicate whether and how you are willing to share photos (your own or ones I take). If you are willing to let me use photos for more than generating discussion during the interview (i.e., in the dissertation or in future publications), please know that people who read about this research could recognize you. Please note that photos are not a necessary part of this study; you are encouraged to participate in this study whether or not you are willing 
to use photos in the interview or share photos and/or be photographed.

I will be the only one with access to the audiotape and the interview transcripts and following the research, they will be stored securely in a safe place at Lakehead University for five years.

Your participation is completely voluntary and you are free to drop out of this study at any point for any reason, with no explanation needed.

If you would like, I can provide you with a short written summary (or even an electronic copy of the full dissertation) after I complete the research. If so, there is a spot on the attached consent form to indicate your interest and your contact information.

If you have any questions or concerns, please feel free to contact me at (807) 983-3331 or gjkuhl@lakeheadu.ca. You may also contact my supervisor, Connie Russell at (807) 3438049 or crussell@lakeheadu.ca. The Lakehead University Research Ethics Board has approved this research. If you have any questions related to the ethics of the research, and would like to speak to someone outside of the research team, please contact Sue Wright, research ethics and administration officer at (807) 343-8283 or research@lakeheadu.ca. Thank you for your participation.

Sincerely,

Gail J. Kuhl

$\mathrm{PhD}$ Student, Lakehead University 


\section{Appendix C: Consent Form}

I, have read the attached

cover letter and am willing to participate in Gail Kuhl's study: LIVING WELL IN A WORLD WITH WOLVES: EDUCATORS' PERSPECTIVES. The purpose of the study has been explained to me and I understand that:

1. Participating in this study is voluntary and I can withdraw from the study at any time for any reason.

2. I may choose not to answer any question I am asked during the interview.

3. There is no apparent risk to my participation in the study.

4. My identity will be protected (unless I request otherwise) and I will remain anonymous in any publication or public presentation of the research findings. However, I realize that if people are familiar with unique aspects of the program where I work, someone may be able to work out my identity.

The pseudonym I would like used in place of my name is:

5. I agree to have the interviews recorded so that transcripts can be made and reviewed at a later point in time.

6. The audiotapes and transcripts (data) from the research will be stored securely at Lakehead University for five years after which time they will be destroyed.

Signature of Participant

Date

Please check if you are interested in any of the following (and, if so, provide your address on the back of this form):

I would like to review the transcript of my interview

I would like a summary of the research once it is completed.

I would like an electronic copy of the entire dissertation once it is complete 


\section{Appendix D: Consent Form for Use of Photographs}

I,

which I have taken and shared, or where I am the subject, may not be used without my written consent. I understand that the use of photographs as discussion points during the interview and/or beyond is optional, and not necessary for my participation in this study.

I give Gail Kuhl permission to use photographs:

(Indicate by checking one or more boxes in which ways you are willing to have photographs used).

a) As discussion points during the interview

b) In her dissertation

c) At a presentation (such as an academic conference)

d) In other publications that might come out of her research (books, papers, magazines)

If you share a photograph that you have taken and are willing to have me use it for any of b) through d) above, do you wish to be credited for the photographs or remain anonymous?

Please indicate only one option from below:

a) I would like to be credited for my photo in any presentation or publication in which it is used, understanding it will sacrifice my anonymity as a participant in the study:

Signature

b) I wish my photo/s to remain anonymous if used either in a publication or presentation:

Signature 